Sign Languages in Village Communities 


\section{Sign Language Typology 4}

Editors

Marie Coppola

Onno Crasborn

Ulrike Zeshan

Editorial board

Sam Lutalo-Kiingi

Irit Meir

Ronice Müller de Quadros

Roland Pfau

Adam Schembri

Gladys Tang

Erin Wilkinson

Jun Hui Yang

De Gruyter Mouton · Ishara Press 


\title{
Sign Languages in Village Communities
}

Anthropological and Linguistic Insights

\author{
Edited by \\ Ulrike Zeshan \\ Connie de Vos
}

De Gruyter Mouton · Ishara Press 
ISBN 978-1-61451-203-5

e-ISBN 978-1-61451-149-6

ISSN 2192-5186

e-ISSN 2192-5194

Library of Congress Cataloging-in-Publication Data

A CIP catalog record for this book has been applied for at the Library of Congress.

Bibliographic information published by the Deutsche Nationalbibliothek

The Deutsche Nationalbibliothek lists this publication in the Deutsche Nationalbibliografie; detailed bibliographic data are available in the Internet at http://dnb.dnb.de.

(C) 2012 Walter de Gruyter, Inc., Boston/Berlin and Ishara Press, Nijmegen, The Netherlands

Printing: Hubert \& Co. GmbH \& Co. KG, Göttingen

(2) Printed on acid-free paper

Printed in Germany

www.degruyter.com 


\section{Contents}

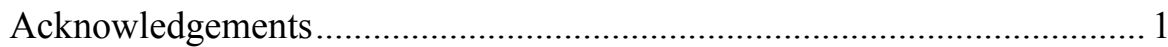

Introduction: Demographic, sociocultural, and linguistic variation across rural signing communities Connie de Vos and Ulrike Zeshan

Part I. Rural signing varieties: Description, documentation and fieldwork practice

Being a deaf white anthropologist in Adamorobe: Some ethical and methodological issues Annelies Kusters.

Colour signs in two indigenous sign languages Dany Adone, Anastasia Bauer, Keren Cumberbatch and Elaine L. Maypilama

Demarcating generations of signers in the dynamic sociolinguistic landscape of a shared sign-language: The case of the Al-Sayyid Bedouin Shifra Kisch

The Kata Kolok perfective in child signing: Coordination of manual and non-manual components

Connie de Vos.

The survival of Algerian Jewish Sign Language alongside Israeli Sign Language in Israel

Sara Lanesman and Irit Meir.

Signing in the Arctic: External influences on Inuit Sign Language Joke Schuit 
An exploration in the domain of time: From Yucatec Maya time gestures to Yucatec Maya Sign Language time signs

Olivier Le Guen....

Deaf signers in Douentza, a rural area in Mali

Victoria Nyst, Kara Sylla and Moustapha Magassouba .....

Language ecological change in Ban Khor, Thailand: An ethnographic case study of village sign language endangerment Angela M. Nonaka

Working with village sign language communities: Deaf fieldwork researchers in professional dialogue

Hasan Dikyuva, Cesar Ernesto Escobedo Delgado,

Sibaji Panda and Ulrike Zeshan

Part 2. Profiles of shared-signing communities

Adamorobe: A demographic, sociolinguistic and sociocultural profile Annelies Kusters

Alipur Sign Language: A sociolinguistic and cultural profile Sibaji Panda.

Algerian Jewish Sign Language: A sociolinguistic sketch Sara Lanesman and Irit Meir. 361

Al-Sayyid: A sociolinguistic Sketch

Shifra Kisch.

Sociolinguistic sketch of Ban Khor and Ban Khor Sign Language Angela M. Nonaka

Chican Sign Language: A sociolinguistic sketch

Cesar Ernesto Escobedo Delgado

Kata Kolok: An updated sociolinguistic profile

Connie de Vos..... 


\section{Contents}

Sociolinguistic sketch of Konchri Sain

Keren Cumberbatch

Sociolinguistic profile of Inuit Sign Language

Joke Schuit

Mardin Sign Language: Signing in a "deaf family"

Hasan Dikyuva

Yolngu Sign Language: A sociolinguistic profile

Elaine L. Maypilama and Dany Adone.

Language index..... 405

Subject index. 408 


\section{Acknowledgements}

This volume is one of the first published outcomes of the EuroBABEL project 'Endangered Sign Languages in Village Communities' (henceforth VillageSign). EuroBABEL is an acronym which stands for Better Analyses Based on Endangered Languages, and this scheme was developed under the EUROCORES programme of the European Science Foundation. The VillageSign project included partner universities from the UK, the US, the Netherlands, Germany, and Israel, and many of the authors represented in this volume have been collaborators in the EuroBABEL initiative. We gratefully recognise the European Science Foundation's contribution as well as the individual national funding agencies who contributed to the EuroBABEL scheme: the Arts \& Humanties Research Council (AHRC), the Deutsche Forschungsgemeinschaft (DFG), the National Science Foundation (NSF), and the Netherlands Organisation for Scientific Research (NWO). Some of the research presented here has been additionally funded by the Endangered Languages Documentation Programme of the Hans Rausing Endangered Languages Project. Other contributions are acknowledged within individual chapters.

We would like to thank the authors who have contributed to this volume within and beyond the EuroBABEL project: Anastasia Bauer, Angela Nonaka, Annelies Kusters, Cesar Ernesto Escobedo Delgado, Dany Adone, Hasan Dikyuva, Irit Meir, Joke Schuit, Kara Sylla, Keren Cumberbatch, Moustapha Magassouba, Oliver Le Guen, Sara Lanesman, Shifra Kisch, Sibaji Panda, Elaine Maypilama, and Victoria Nyst. It has been a privilege to work with you all. We would also like an opportunity to express our gratitude to our peers who reviewed the chapters including the editorial board members of the Sign Language Typology Series, and the series editors Marie Coppola and Onno Crasborn.

We are also indebted to the dedicated team of people who have contributed to the copyediting, typesetting, design, and production of this volume through all its stages, in particular Robin Arora, Toni Comer, and Jennifer Webster at Ishara Press, and Marcia Schwartz and Angelika Hermann from De Gruyter Mouton.

Connie de Vos

Ulrike Zeshan 


\section{Introduction: Demographic, sociocultural, and linguistic variation across rural signing communities}

\section{Connie de Vos and Ulrike Zeshan}

This book unites the work of both anthropologists and linguists who have conducted fieldwork in rural signing communities around the globe. In most cases, these signing communities have emerged in response to a high incidence of (often hereditary) deafness. In contrast to the national sign languages used in urban deaf communities, these indigenous sign languages are typically shared between deaf and hearing community members, thus facilitating a high degree of integration between deaf and hearing individuals. This volume represents the largest collection of comparative work across such "deaf villages" to date.

There have been sporadic publications on these communities over the past few decades (see for instance Kakumasu, 1968; Washabaugh, 1979; Groce, 1985), but the chapters in this volume constitute the first extensive compilation of academic papers regarding these signing varieties and the communities in which they have emerged, from both anthropological and linguistic perspectives. Moreover, for some of the signing varieties discussed here, this is the first printed publication to appear (see the community sketches by Dikyuva; Lanesman \& Meir, and Panda in Part II of this volume).

All known village sign languages are endangered, usually because of pressure from larger urban sign languages, and some have died out already. Ironically, it is often the success of the larger sign language communities in urban centres, their recognition and subsequent spread, which leads to the endangerment of these small minority sign languages. For this reason the book also addresses this specific type of language endangerment, documentation strategies, and other ethical issues.

The sections below serve as an introduction to the demographic, sociocultural, and linguistic diversity that is represented in this book. Results from the chapters of this volume are contextualised by describing some commonalities across the various sites and languages, as well as, most importantly, highlighting the unique findings reported in each of them. 


\section{The social dynamics of rural signing communities}

The notion of a "deaf village" is closely related to the concepts of a "sharedsigning community" (Kisch 2008), an "assimilative Deaf community" (Groce 1985), and a "speech/sign community" (Nonaka 2007). Alternative terms in the literature for village sign languages are "indigenous sign language" (Woodward 2003; Nonaka 2009) and "rural sign language" (de Vos 2011). Moreover, a term sometimes related to village sign languages, but nonetheless distinct, is "emerging sign language," used to indicate a broader category of sign languages that have emerged within the last two or three generations (Padden 2010). Each of the latter terms underscores a different aspect of sign languages that have emerged in rural communities.

The table below is based on the community sketches in Part II of this volume and summarises a few of the relevant dimensions along which "deaf villages" may vary. The variation found across these communities suggests that "deaf villages" are far from homogeneous. Moreover, as Nonaka (this volume) points out, dichotomies may be motivated by whichever dimension is taken to be relevant to the phenomenon under consideration. With this in mind, this volume has taken a liberal approach to terminology, and the above terms are used largely interchangeably, until we arrive at better-informed classifications.

A number of differences between the communities discussed here stand out in particular. First of all, the rural signing communities featured in this book often do not constitute the classical "deaf village" scenario. Mardin Sign Language was never used by the entire town of Mardin, but has rather functioned as the family sign language for the Dilsiz family and their particular social networks in Mardin. The Turkish word dilsiz means 'deaf', which is perhaps unsurprising given the fact that this family has had deaf members for the past four generations. The emergence of the AJSL signing community in Ghardaia, Algeria was followed by successive waves of emigration in the 1940s and 1950s. While Algerian Jewish Sign Language (Lanesman \& Meir, this volume) thus first arose in a Jewish enclave of Algeria, the community has been in a state of diaspora and its members are now dispersed in Israel and France. Yolngu Sign Language (Maypilama \& Adone, this volume) stands out from the other village sign languages described in the literature, as it is better known as the "alternate sign language" of the Yolngu aboriginal community. Within multiple aboriginal communities, alternate sign languages are used in situations of speech taboos, for instance during mourning, or during hunting (Kendon 1988). Within the Yolngu case, this signed form of communication 


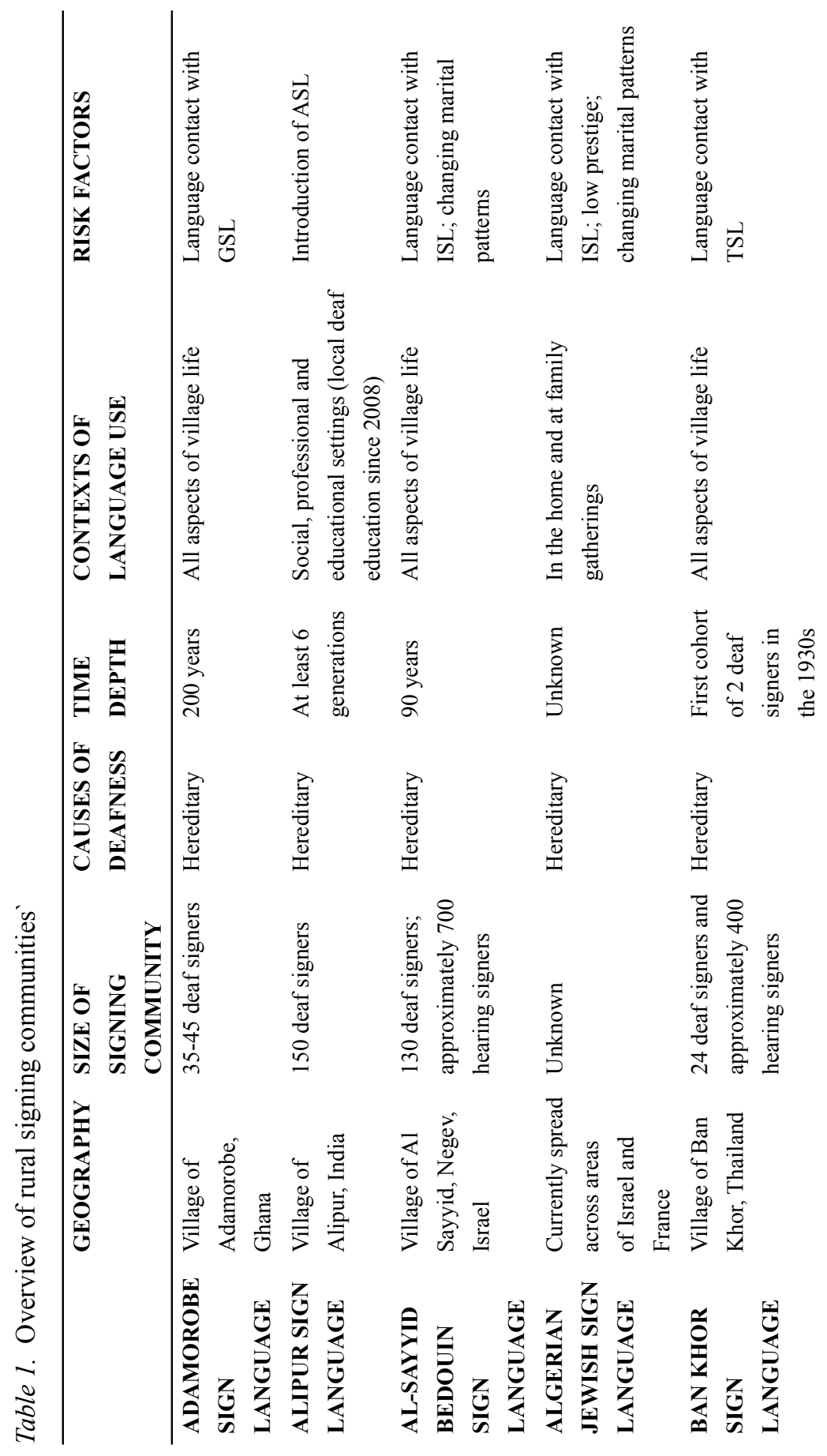


Variation across rural signing communities 5

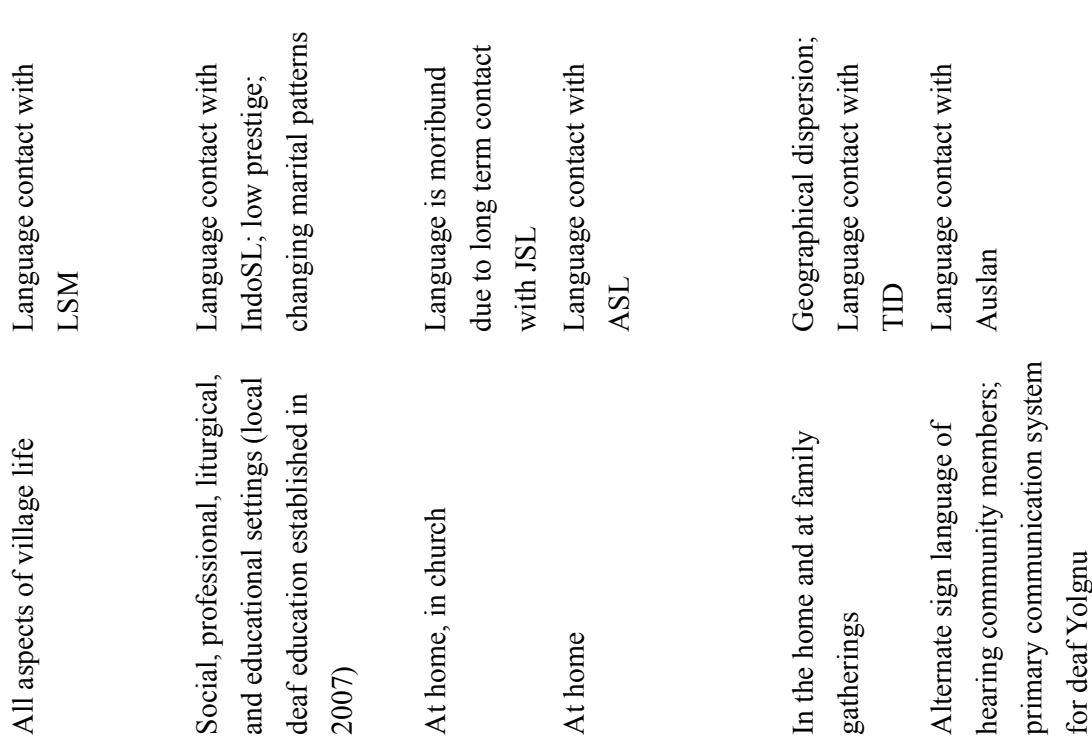

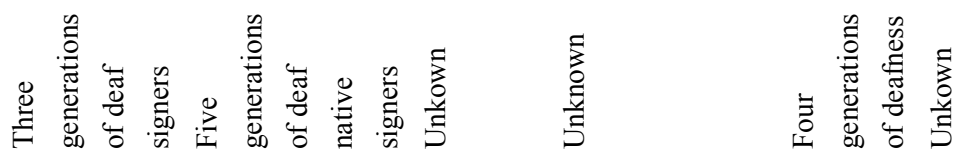

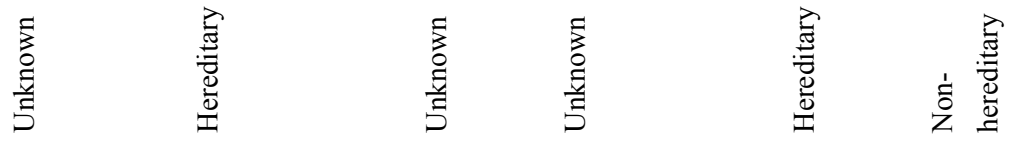

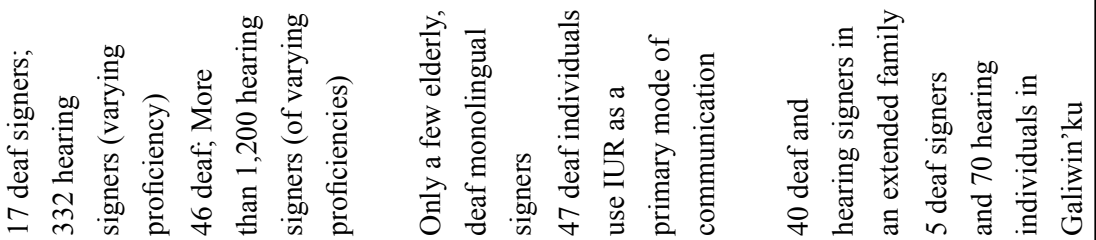

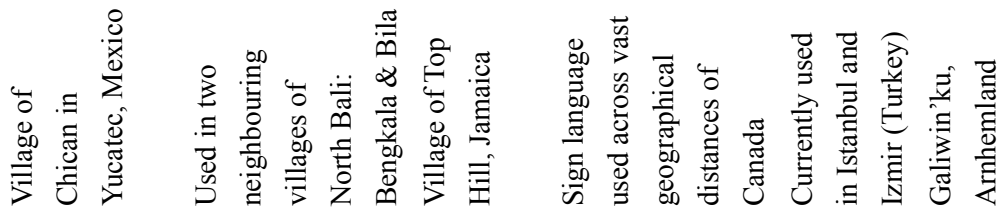

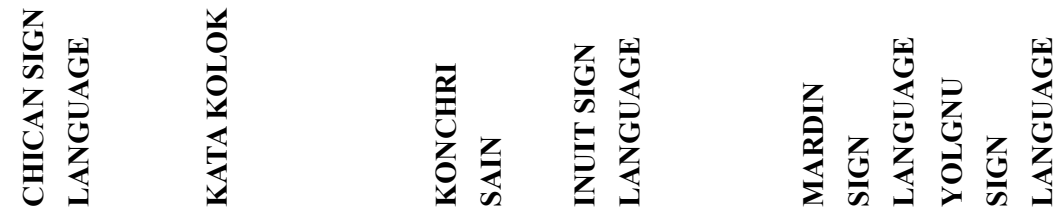


has also been adopted by a small group of deaf individuals who use it as their primary means of communication, and there has not been a systematic study of the potential contrasts between these two domains of use to date (Maypilama \& Adone, this volume). The origins and modes of transmission of Inuit Sign Language are as yet unclear (Schuit, this volume), but its vast geographical spread suggests that it might have emerged from a form of gestural communication shared by hearing Inuit, perhaps even a trading language such as Plains Indian Sign Language (Davis 2010).

The dichotomy between urban and rural sign languages is primarily based on their distinctive origins: segregated formal deaf education, and informal shared sign language use, respectively. The above cases, however, indicate that rural signing varieties may be differentiated even further in terms of their historical development and geographical spread. Furthermore, Nyst (this volume) notes that our current jargon is inadequate for identifying the wider range of signing varieties that exists in rural Mali. Some of the sign languages found in Africa (e.g. Bamako Sign Language of Mali) have emerged outside the context of formal deaf education, but within extensive urban networks. Future classifications and comparisons of these different types of signing varieties could lead to a deeper understanding of the relationship between social dynamics of signing communities and their linguistic structures. For instance, section 2 of this introduction touches upon cross-modal contact between the types of sign languages and the spoken languages that surround them.

A final issue which arises from the variation reported in the community sketches is that, although the majority of sign languages under consideration here are used in delineated "villages", this term has little descriptive value from an anthropological point of view. This is particularly evident when comparing the demographic figures of the "villages" of Alipur, with 20,000 individuals, and Chican, which has a population of 720 . Alipur village also stands out as one of the wealthier communities with a flourishing gem-stone industry, which has enabled the construction of large buildings and the establishment of a locally-funded deaf school. As noted by Nonaka (this volume), most of the communities under consideration here have labour-intensive economies which include agricultural activities, and in most communities, deaf and hearing community members hold similar occupations. However, differential education opportunities allow hearing villagers to hold professional jobs outside the community additionally (Escobedo Delgado, this volume).

The communities listed here are characterised by the existence of a sign language shared between deaf and hearing community members. The use of signed communication in everyday activities appears to facilitate a high degree of integration of deaf and hearing community members. The 
communicative ease with which deaf individuals function within these rural communities has sometimes led to a naive conception of these villages as Deaf utopias (see Kusters, 2010; this volume). The sketches presented in Part II of this volume aim to fully appreciate the various sociocultural adaptations and views on deafness that are held within these communities.

The differential social construction of deafness in these communities becomes particularly clear in the domain of partner choice and wedding arrangements. In the villages of Bengkala and Chican, deaf individuals are free to choose a deaf or hearing spouse and both deaf-deaf and deaf-hearing marriages are attested (Marsaja 2008; Escobedo Delgado, this volume). In Adamorobe, deaf individuals have not been allowed to marry each other since 1975, because these marriages invariably led to deaf offspring (Kusters, this volume). In Alipur, wedding arrangements traditionally involve financial transactions, but there are significant differences in the costs between hearing and deaf-hearing weddings. The family marrying off a deaf woman pays a higher dowry than if they had had a hearing daughter. Furthermore, hearing men do not pay dowries when they marry a deaf woman, while deaf men do (see Panda, this volume for details). In the case of Al-Sayyid, arranged marriages are also the norm, and all of those marriages were mixed, until a deaf Al-Sayyid woman married her deaf classmate from outside Al-Sayyid in 2004. Since then there has been a steep increase of deaf Al-Sayyid women marrying deaf men from elsewhere, a process which has been facilitated by networks that, being based in educational settings, include the wider Israeli deaf community (Kisch, this volume). Similarly, in the case of Bengkala, attendance at deaf schools in other parts of Bali has led to increased contact with the wider Balinese deaf community, and subsequently a larger number of marriages between deaf men and women from Bengkala with deaf individuals who are not from this village. As deaf individuals from outside of these villages are unlikely to carry the same recessive gene causing deafness within these communities, such couples do not usually bear deaf offspring. Consequently, these changing marital patterns may reduce the incidence of deafness within these villages, and threaten the continued use of the indigenous sign language in the long run (de Vos, this volume).

As the rightmost column of Table 1 illustrates, the rural sign languages included in this volume are all either endangered or at risk of becoming endangered to varying degrees. Apart from changing marital patterns, language contact with the urban sign languages of national deaf communities appears to be a major risk factor. In the village of Ban Khor, Thai Sign Language is associated with increased educational and professional opportunities and better access to sign language interpreting, and therefore enjoys 
a higher prestige than Ban Khor Sign Language. Over the course of a few years only, deaf signers have started to replace BKSL signs with Thai signs and are adopting signs for new concepts as well (Nonaka, this volume). A particularly interesting observation with respect to this type of endangerment is that in many of the communities represented in this volume, it is often the hearing signers who are most conservative, and who could be regarded as the safekeepers of these shared sign languages (see the contributions by de Vos; Dikyuva; Lanesman \& Meir; and Nonaka).

\section{The typological contribution of sign languages from rural signing communities}

Having looked at the considerable demographic and sociocultural variability of sign languages in rural communities, we now turn to some of the interesting linguistic properties of the sign languages represented in this volume. Village sign languages are one of the very latest additions to the body of knowledge in sign language linguistics. Since some of these sign languages have had limited contact with other sign languages in their formative stages, and their sociolinguistic characteristics are so strikingly different from the better-known sign languages in urban deaf communities, it is not unreasonable to expect that investigating the linguistic structures of the sign languages may lead to important new discoveries.

The rationale for expecting village sign languages to add significantly to our appreciation of typological variability across sign languages is first explored in more detail in section 2.1. We then take a closer look at the linguistic and typological significance of data from village sign languages, summarising what is known so far and how these data can be situated in the wider context of sign language linguistics (section 2.2). Finally, we consider the question whether village sign languages can be said to constitute a linguistic sub-type in contrast with urban sign languages (section 2.3). Where appropriate, reference is made to the individual chapters in this volume.

\subsection{Village sign languages and Sign Language Typology}

The systematic comparative study of sign languages is known as Sign Language Typology (Zeshan 2004a, 2004b). This area of inquiry has become 
possible over the past decade because data from genetically and geographically diverse sign languages is increasingly becoming available for comparative studies. Large-scale comparative studies across sign languages have been undertaken for the domains of negatives and interrogatives (Zeshan 2006), possessive and existential constructions (Zeshan \& Perniss 2008), and semantic fields (Zeshan \& Sagara, in prep.). The latter two publications include contributions on village sign languages.

It has been argued in Zeshan (2007) that our understanding of typological diversity across sign languages resembles a mosaic where new pieces are constantly being added. From the 1960s and 1970s onwards, sign language research was initially dominated by work on American Sign Language, and the largest body of literature still relates to this language. Several Western European sign languages, such as British Sign Language and German Sign Language (DGS) are also relatively well-documented by now. More recently, important work has been carried out on non-Western sign languages in urban deaf communities, such as, for instance, in Jordan (Hendriks 2008), Hong Kong (Tang \& Gu 2006), India (Zeshan 2000), Turkey (Özyürek, Zwitserlood, \& Perniss 2010), and Uganda (Lutalo-Kiingi forthcoming), among others. With each successive "wave" of new data, we are able to gain a clearer understanding of sign language structures around the world and to reset our perspective, which was initially skewed by emphasis on Northern American and Western European sign languages.

A parallel process of discovery has taken place in spoken language linguistics, in particular the typology of spoken languages, from the 1970s onwards. Many "exotic" languages have provided data on structures that are unusual or entirely absent from the previously prototypical Indo-European languages. For instance, recognition of mirativity and evidentiality as a grammatical category depended crucially on evidence from various "exotic" spoken languages (De Lancey 1997, Aikhenvald 2003). A similar development, albeit delayed by several decades, can now be expected for sign languages.

Thus village sign languages represent the latest addition to the mosaic of sign language structures. Detailed information about some of the linguistic structures of these sign languages has only just become available over the past few years (e.g. Nyst, 2007; Marsaja, 2008; de Vos, 2012), and their significance for comparative purposes is already apparent from such initial studies. In particular, the field of Sign Language Typology benefits immensely from data on these sign languages, and there are important conclusions for other areas of linguistic inquiry too (see section 3 ). 


\subsection{The significance of linguistic data from village sign languages}

Although linguistic data from village sign languages is still relatively scarce, it has become abundantly clear already that these sign languages extend our knowledge of the linguistics of sign languages in several ways. Thus we find that village sign languages exhibit many unique structures not documented in any urban sign languages so far, as well as present counter-examples to previously presumed universal tendencies in sign languages.

A particularly important discovery that has been made in several village sign languages is that the organisation of the "signing space", i.e. the conventional space around the body use for linguistically relevant expressions during signing, is much more cross-linguistically diverse than previously thought. It has long been assumed in sign language linguistics that the rich array of grammatical spatial structures that characterises sign languages is instantiated in a very similar way in all languages in the visual-gestural modality. Constructions designating the movement and location of various categories of entities, known as "whole entity classifiers" have been shown to have a great degree of structural homogeneity across sign languages (Eccarius \& Brentari 2007). Spatial verb agreement or "directionality", where the direction of hand movement indicates the grammatical / semantic roles of arguments equivalent to subject-object agreement markers in spoken languages, has been documented in many urban sign languages so far (e.g. Padden 1988; Engberg-Pedersen 1993; Mathur \& Rathmann 2006; Hong 2009). Both of these construction types rely on spatial conceptualisations projected onto the signing space, and it is important to localise discourse referents in signing space in order to build up a notional "stage" on which discourse participants can be "placed" to act and interact. It has been argued that the semi-conventionalised contact pidgin of International Sign (IS) includes grammatically rich spatial structures because these are shared between the sign languages that IS has originated from (Supalla \& Webb 1995).

However, data from village sign languages present counter-evidence to the notion that spatial structures such as directionality and classifier constructions could be universal across sign languages. Table 1 lists some features of spatial grammar across different sign languages. "X Sign Language" could stand for any previously documented urban sign language, such as Japanese Sign Language, American Sign Language, Brazilian Sign Language or Turkish Sign Language, as they all have both directional verbs and whole entity classifiers. However, the situation is different in the two village sign languages Adamorobe Sign Language (AdaSL) from Ghana and Kata Kolok 
(KK) from Bali. For AdaSL, Nyst (2007) presents evidence that whole entity classifiers are not present in this language. In fact, the entire system of projecting referent entities on to the signing space in front of the signer in a "stage-like" way is not used in AdaSL. Instead of this otherwise common so-called "observer perspective" that takes a bird's eye view on the situation being described, AdaSL only uses a "character perspective" where everything is narrated from the point of view of the signer's own body (see Perniss 2007 on the distinction between the observer perspective and character perspective). AdaSL does make use of directional verbs in its grammar.

Conversely, KK has a well-developed system of whole entity classifiers, with some particularities in the use of handshape and movement patterns found in this category of signs (Marsaja 2008). Instead, KK has no systematic grammatical category of directional verbs (de Vos, 2012). This is due to a radically different organisation of grammatical signing space in $\mathrm{KK}$. Unlike all other known sign languages, KK signers do not establish conceptual referent locations (known as "loci") in signing space. Instead, referents are localised in discourse according to their real-world locations. For instance, in order to refer to a person, $\mathrm{KK}$ signers will point to the actual physical location of the person's home in the village, rather than pointing out an arbitrary location in signing space, as is done in other sign languages. As the full grammatical use of the directionality depends on setting up conceptual loci for referents in space that can then be used as beginning and end points of the direction of verb form, KK lacks a system of directionality in verbs (de Vos 2012).

Table 2. Comparing features of spatial grammar

\begin{tabular}{llll}
\hline $\begin{array}{l}\text { Features of spatial } \\
\text { grammar }\end{array}$ & $\begin{array}{l}\text { X Sign } \\
\text { Language }\end{array}$ & $\begin{array}{l}\text { Adamorobe Sign } \\
\text { Language }\end{array}$ & Kata Kolok \\
\hline Directional verbs & YES & YES & NO \\
Whole entity classifiers & YES & NO & YES \\
\hline
\end{tabular}

AdaSL and KK thus not only present a challenge to presumed sign language universals that were posited on the basis of urban sign languages, but these two village sign languages also differ from each other in their spatial organisation. This is an important point, as it is crucial to avoid premature overgeneralisations about "urban" and "rural" sign languages. We need to consider the empirical evidence from individual rural sign languages in much detail before any inductive generalisations can be drawn from such data. 
Village sign languages may not only lack structures found in other sign languages, they may also include structures that have never been documented in other sign languages before. Data that provide evidence for unique structures that are new to sign language linguistics are particularly valuable for comparative research such as conducted in Sign Language Typology studies. Such structures extend the known range of variation across sign languages and may also provide new insights into typological patterns. An interesting example of such data comes from the domain of number signs in different village sign languages. Zeshan et al (in prep.) describe the systems of cardinal numerals in three village sign languages from India (Alipur Sign Language, APSL), Turkey (Mardin Sign Language, MarSL), and Mexico (Chican Sign Language). All three sign languages have numeral systems with unusual features that have not been documented in any other sign languages yet. Zeshan et al (in prep.) describe the occurrence of vigesimal numerals, subtractive numerals, unusual numeral bases, and spatial morphology in numerals. Table 2 presents a summary of these structures across the three sign languages.

Table 3. Typologically unusual numerals in village sign languages

\begin{tabular}{lccc}
\hline & ALIPUR SIGN & CHICAN SIGN & MARDIN SIGN \\
& LANGUAGE & LANGUAGE & LANGUAGE \\
\hline Base-20 numerals & - & + & + \\
Base-50 numerals & + & + & + \\
Subtractive & + & - & + \\
numerals & & - & - \\
Spatial & + & & \\
modification & & & \\
\hline
\end{tabular}

As is evident from Table 2, each sign language uses a different array of structures. ${ }^{1}$ Vigesimal numerals, which typically involve multiplication with 20 , are not uncommon across spoken languages (cf. Comrie 2005), but had not been documented in sign languages before. In MarSL, there is a vigesimal subsystem whereby 40 is expressed as $2 \times 20,60$ as $3 \times 20$ and 80 as $4 \times 20$ (see Figure 1). 

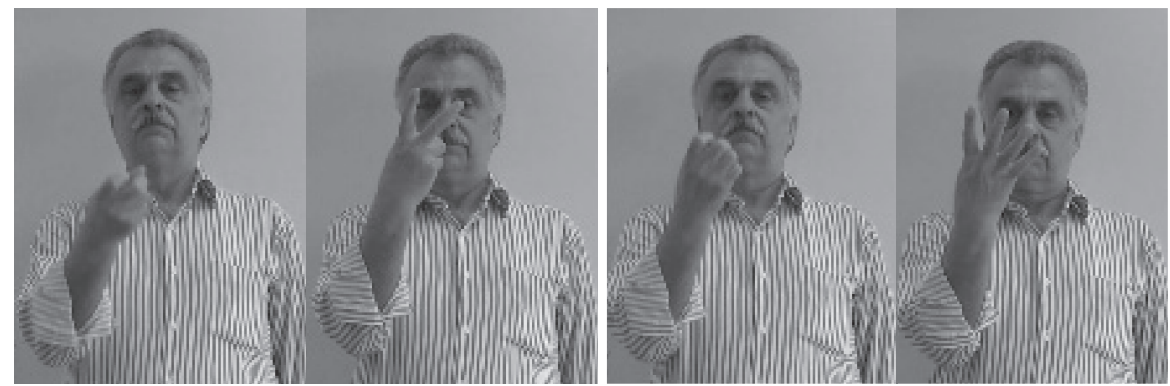

Figure 1. The numbers 40 and 80 in MarSL

The number 20 is also used, along with the number 50, as a base from which to construct larger numerals. In Chican Sign Language, these numbers are added successively, so that, for instance, 80 is expressed as $50+20+10$. Thus 50 and 20 are both used as additive bases in Chican Sign Language (see Figure 2). It is striking that independently of each other, all three sign languages have developed a system that uses 50 as a base number.
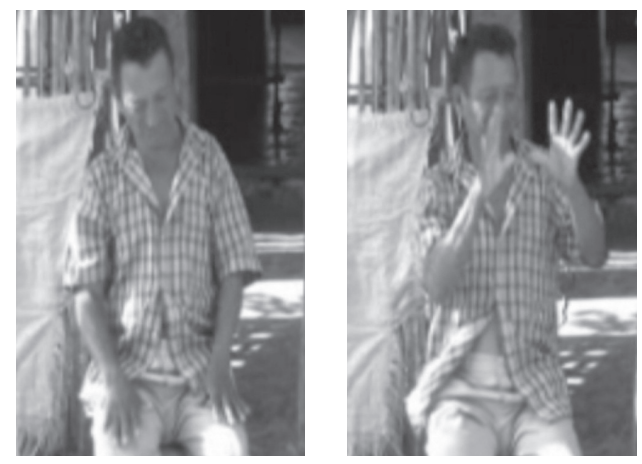

Figure 2. $20+10$ in Chican Sign Language to express the number 30

Figure 3 shows the use of spatial modification in a numeral subsystem in APSL. This is used to express the numbers 100, 1,000 and 100,000; that is, increasing the spatial dimensions of the sign is equivalent to adding additional zeros in written numbers. ${ }^{2}$ Obviously, spatial modification is not available in the morphology of spoken languages, and to our knowledge, an equivalent construction is also undocumented in other sign languages. 

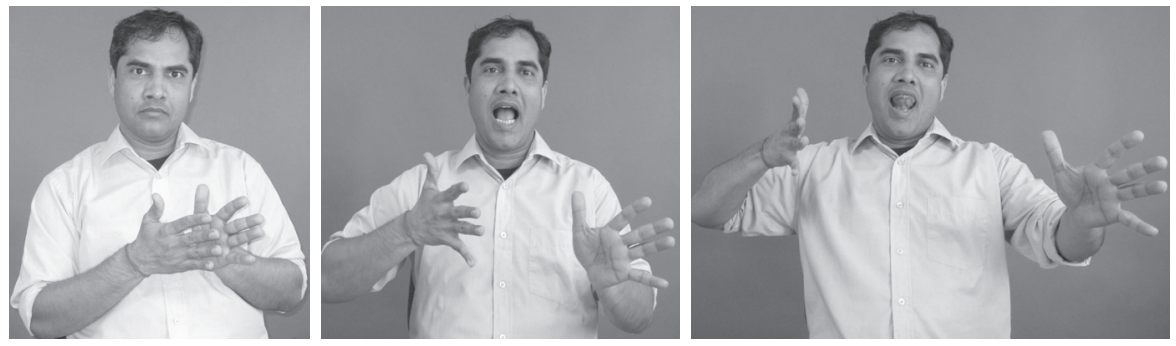

Figure 3. The numbers $100,1,000$ and 100,000 in APSL

Both MarSL and APSL also use subtractive numerals, though in quite different ways (see Zeshan et al., in prep, for details). In MarSL, a number such as 18 may be expressed as 20-2 (see Figure 4). The subsystem in MarSL has a more restricted scope of use (up to a maximum of -5 ), but subtractive numbers in APSL are much more productive, with numbers such as 30-2 for $28,200-5$ for 195 , or $50-2$ for 48 found with some frequency in the data. Subtractive numerals are known to occur in some spoken languages, but were previously undocumented in sign languages.
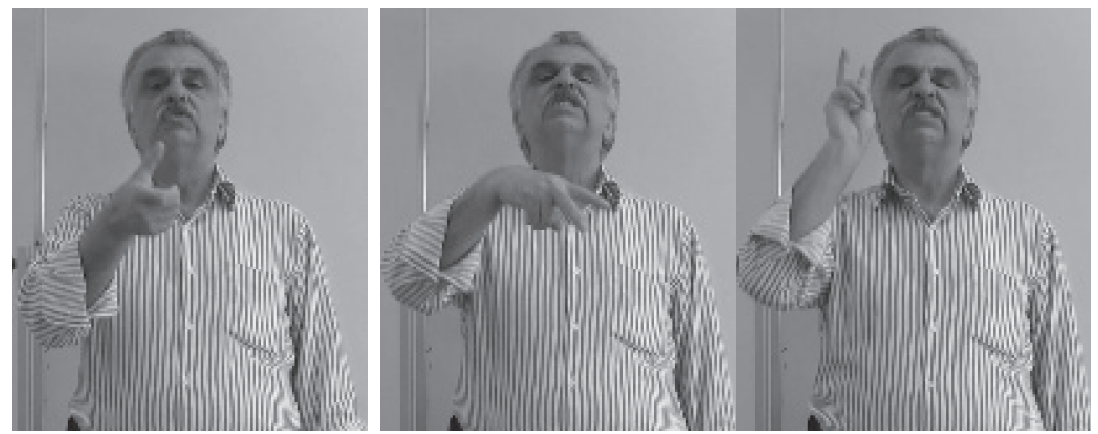

Figure 4. TWENTY TWO-LESS in MarSL to express the number ' 18 '

These data on numerals extend considerably our understanding of the range of typological variation that can be found across sign languages in this domain. It is abundantly clear that village sign languages are a rich source of new information on the possible linguistic expressions that can be found in sign languages. They challenge previously held assumptions on the structures we previously expected to find in all sign languages, as well as presenting evidence of structures that are new to sign language linguistics. As some of the latter structures are attested or even common in spoken languages, such data may also lead us to reconsider the relationship between signed and spoken languages. That is, with respect to a particular domain of phenomenon, a certain sign language may turn out to be more similar to a spoken 
language than to other sign languages. Therefore, it is desirable to evolve a new approach to typology that is explicitly and systematically cross-modal.

\subsection{Village sign languages and urban sign languages}

It may be tempting to think of sign languages in terms of two distinct subtypes - village/rural sign languages and urban sign languages. Many rural sign languages tend to have several aspects of their sociolinguistic setup in common. For instance, deaf education and other specific infrastructure such as sign language interpreting or deaf associations tend to be absent, a large number or even the majority of sign language users are hearing people, the incidence of deafness has genetic reasons, and there is typically no official status or recognition for rural sign languages. Lanesman and Meir's contribution in this volume sets out these parameters very clearly, including the important role that hearing signers have played in the maintenance of Algerian Jewish Sign Language so far, and there are pertinent similarities with a number of other village sign languages. However, as argued in Section 1 and demonstrated in more detail in the sociolinguistic sketches in Part II of this volume, there are also many differences between the various rural signing communities. It is far from correct to say that all village sign languages occur in very similar sociolinguistic or socio-cultural settings.

In a similar way, there is limited evidence that certain kinds of linguistic structures tend to occur in several unrelated rural sign languages. For example, it has been reported that the conventional signing space is very large in several village sign languages. Signing with fully outstretched arms, bending down for signing in a lower space, and a wider range of places of articulation on the body such as on the lower extremities have been documented (cf. Marsaja, 2008 and de Vos, 2012, for Kata Kolok; Nyst, 2007 for Adamorobe Sign Language). However, as illustrated in the previous section, there are also many grammatical differences between village sign languages, as would be expected given that they have no geographical or genetic affiliation with each other.

Similarly, the organisation of the lexicon is obviously particular to each individual sign language, regardless of possible parallels between village sign languages in individual instances. For instance, it has been observed that rural sign languages sometimes seem to have a relatively smaller number of items in semantic fields, such as pointed out in Adone, Bauer, Cumberbatch and Lawurrpa with respect to colour terms in this volume. Some village sign languages have very few colour terms and use alternative ways of referring 
to colour, in particular pointing in the environment. Typically, such pointing is not ad hoc, but conventionalised in some way: In Al-Sayyid Bedouin Sign Language, colourful clothing is used to point out individual colours, and in Kata Kolok, pointing for colour is accompanied by a conventionalised nonmanual behaviour or the lexical sign for PAINT (de Vos, 2011). Again, one could speculate whether the absence of formal schooling has an impact on lexicon areas such as colours or large numbers (some village sign languages, such as Alipur Sign Language, lack signs for specific very large numbers), but on the basis of data available so far, this does not warrant a categorisation of "village sign languages" as a linguistic sub-type. In fact, it is necessary to look much more closely at the characteristics of individual instances of rural sign languages.

The contribution by Nyst in this volume provides a particularly telling example in detailing the use of rural signing varieties in various communities in Mali. Nyst argues that more careful distinctions need to be made between what has been known previously as "home sign", that is, the improvised gestural communication used by isolated deaf people to communicate with their hearing environment, and the "full-fledged sign languages" of large, typically urban communities of deaf signers. Many scenarios of sign language use in rural areas do not fit neatly into these two categories, but fall somewhere in between, and this in-between area has not been adequately conceptualised in sign language linguistics. ${ }^{3}$

One suggestive generalisation that merits more detailed consideration is the fact that many village sign languages exist in a language contact situation that is different from urban deaf communities. While all sign languages are subject to language contact with the surrounding spoken languages, the rural communities represented in this volume are different in that deaf signers live in close daily contact with a large number of hearing signers, who represent the majority of sign language users. That is, the majority of signers use the sign language as a second language (L2), and it is legitimate to ask whether this has effects on the linguistic organisation of the sign language. For example, the comparatively limited use of verb inflection in ABSL (Sandler et al. 2005), Adamorobe Sign Language (Nyst 2007), Kata Kolok (Marsaja 2008; de Vos 2012), and IUR (Schuit et al. 2010), may have some- thing to do with the intensive bimodal language contact situation, the learnabilty of morphologically complex structures in spatial grammar by adult learners, or both. Again, this is first and foremost an empirical question, and we must be careful not to draw premature conclusions.

The existing data so far suggest that the impact of spoken language structures on village sign languages is variable. Nyst (2007) documents several 
important structural effects of the presence of spoken Twi in the environment of Adamorobe Sign Language. This includes the role of mouthing (mouth movements derived from the articulation of spoken language words) in distinguishing colour terms, in a similar way as in Konchri Sain (see Adone, Bauer, Cumberbatch \& Lawurrpa, this volume). AdaSL also has serial verb constructions that parallel the serial verb constructions in Twi. On the other hand, Kata Kolok shows virtually no grammatical influence from spoken Balinese (Marsaja 2008). There are virtually no mouthings in KK, and parallels in grammatical constructions are negligible. Similarly, Zeshan et al. (in prep.) detail a mismatch between number systems in the signed and spoken languages of Mardin (Turkey), Alipur (India) and Chican (Mexico). In all three sign languages, the way numbers are constructed is very different from the surrounding spoken languages. For instance, none of the spoken languages used in Mardin at the time when the sign language community first flourished - Turkish, Kurdish, and Arabic - has any occurrence of vigesimal or subtractive numbers. On the basis of available evidence so far, it must be concluded that the impact of the large number of hearing signers on the linguistic structures of rural sign languages varies according to factors that are not yet sufficiently clear.

What is very clear in all village sign languages where this has been investigated is the influence of local gestures used by hearing people on the sign language. For instance, functional gestures used for questions, non-manual gestures such as negative head movements (e.g. headshake), and "word-like" gestures such as "thumbs-up", "money", etc., are easily carried over into sign languages. The chapter by Le Guen in this volume presents a detailed example of signs used to express time in Yucatec Mayan Sign Language, ${ }^{4}$ and how these signs are derived from the conversational gestures used by hearing people in the same area. Both conventional gestures and signs in the local sign language reflect the conceptualisations of time that are characteristic of this cultural context, where time is viewed as cyclical rather than as a linear succession of events. The influence of gestures on signs is not surprising, and in fact, is amply documented in urban sign languages (e.g. Zeshan 2000 for Indo-Pakistani Sign Language). Therefore, the relationship between gestures and signs is not something that distinguishes rural and urban sign languages from each other, but something that is characteristic of both.

Another potential issue that has sometimes been suggested to account for structural characteristics of rural sign languages is their assumed developmental path. Nonaka (2004) mentions that sign languages in small-scale rural communities often tend to arise suddenly, flourish briefly, and disappear relatively quickly, often before there has been a chance to properly document 
them. There is clearly a developmental difference between an ad hoc home sign system used by the first deaf person born into a community and a conventionalised sign language used by a substantial number of people over several generations. Sandler et al. (2005) imply that the absence of a developed system of spatial verb agreement (directionality) in Al-Sayyid Bedouin Sign Language (ABSL) could be due to the fact that this sign language has arisen quite recently. As the contribution by Kisch in this volume details, the first deaf persons in the Al-Sayyid community were born from the 1920s onwards, and it took until the 1950s for a larger cohort of deaf signers to arise, who would have had sign language input from older signers other than home sign. A possible argument is therefore that the historical development of ABSL has not yet progressed far enough to produce a full-fledged system of verb directionality.

However, evidence from other village sign languages is not in line with an argument around a historical developmental path. The case of Kata Kolok is pertinent here as this sign language also lacks a developed system of directionality. However, KK is considerably older than ABSL. De Vos (this volume) argues that a substantial cohort of deaf signers was probably already present in the community five generations ago. ${ }^{5}$ Yet KK also lacks verb directionality, and there is no indication that generations of younger signers are moving towards such a system. In other words, KK has historically stable and fully developed sign language without spatial verb agreement. Similarly, Nyst (2007) concludes that AdaSL seems to have moved on its own particular developmental path, and characteristics that are common in urban sign languages, such as entity classifiers, have simply not been part of the development of AdaSL.

In conclusion, it is premature at the current stage of our knowledge on rural sign languages to make unwarranted generalisations about their linguistic structures, their historical development, and the relationship between linguistic structures and sociolinguistic characteristics. In order to make valid empirical generalisations, we first need to assemble data from a much larger number of rural sign languages. Data from each sign language should be evaluated carefully and on their own terms in each case. This approach has been integral to the field of Sign Language Typology from the beginning, and has resulted in surprising discoveries, for instance with respect to typological variation across sign languages in the domains of negation, questions, and possession (Zeshan 2006, Zeshan and Perniss 2008). There is no doubt that data on village sign languages will extend further our appreciation of typological diversity and patterning in sign languages. 


\section{Conclusion}

Perhaps the most important contribution that this volume will make to the field of sign language studies is the sheer variety of topics that can fruitfully be addressed in relation to village sign languages. We can look at these sign languages from the point of view of language endangerment, as in the chapter by Lanesman and Meir. The great majority of village sign languages are moderately or critically endangered, and there is a clear urgency in documenting both their unique sociocultural settings and their linguistic structures. The literature on language endangerment so far comprises virtually no studies of endangered sign languages, so material such as is assembled in this volume makes an innovative contribution to the field of language endangerment. Another important angle is the considerable variety of settings that we find across rural languages. The contribution by Nyst rightly points out that our conceptual understanding and terminology in sign language linguistics are currently insufficient to properly appraise and reflect on these various situations. Moreover, the various sociolinguistic settings in which these signing varieties arise call for flexible and alternative language documentation methods (contributions by Dikyuva, Escobedo Delgado, Panda and Zeshan; Nyst, this volume; de Vos 2012).

A particular hallmark of the present volume is the fact that both linguists and anthropologists have contributed to its content. The detailed reflections by Kisch on how the ABSL community of signers has arisen and constructed itself will allow sign language linguists to think about these communities in a much more differentiated way. Conversely, the contribution by de Vos is one of very few studies of first language acquisition in a rural sign language, and such material is helpful to consider for anthropologists with an interest in the transmission of language and culture. Researchers from allied specialisms such as gesture research, linguistic anthropology, and multimodal communication may also find the multi-layered relationships between gestures and signs valuable. The chapter by Le Guen presents a fine-grained analysis of the expression of time, and the YMSL system is clearly very different from what we find in most urban sign languages.

Finally, several contributors in this volume also reflect on the role of researchers in these rural communities. This is seen in the chapter by Kusters, which documents and reflects on her work in the AdaSL community. The contribution by Dikyuva, Escobedo Delgado, Panda and Zeshan compares work in three different fieldwork settings in Turkey, India and Mexico, based on the first-hand experiences of the three deaf fieldwork researchers who are co-authors of the contribution. Laid out in the form of professional 
dialogues and interviews, this material is a step towards detailed case studies on research practices and ethics in rural communities of sign language users. Such issues have always been at the forefront of attention for the research teams that have been involved in these first large-scale comparisons of village sign languages, with all their intriguing sociocultural and typological differences.

\section{Notes}

1. Additional structures are used in the numeral systems of each of these sign languages that are commonly found elsewhere, such as numeral incorporation. These are omitted here as the focus is on the unique structures of village sign languages, but the full range of structures is reported in Zeshan et al (in prep.).

2. There is no particular sign for 'million', as the system is modelled on the surrounding spoken language Urdu, which has a separate word for ' 100,000 ' but not for 'million'.

3. Zeshan (2011) uses the term "communal home sign" to refer to in-between situations along a continuum from "home sign" to "sign language", but it is clear that a larger number of distinctions need to be made.

4. The sign language used in Chican, where Le Guen's data are from, is called Chican Sign Language in the sociolinguistic sketch by Escobedo Delgado in this volume, but is called Yucatec Mayan Sign Language by Le Guen.

5. Like Kisch in this volume, de Vos is aware that the definition and delineation of "generations" is difficult in both village communities. However, different delineations would still lead to the same conclusion that the incidence of deafness is substantially older for the KK community, and is probably in line with many present-day urban sign language communities, particularly in those regions in developing countries where urbanisation is more recent than in industrialised countries.

\section{References}

Aikhenvald, A.Y.

2003 Evidentiality in tyological perspective. In: Aikhenvald, A.Y. and Dixon, R.M.W (eds.): Studies in Evidentiality. Typological Studies in Language vol. 54. Amsterdam: John Benjamins.

Davis, J. E.

2010 Hand Talk. Cambridge: Cambridge University Press. 
DeLancey, S.

1997 Mirativity: The grammatical marking of unexpected information. Linguistic Typology 1.33-52.

de Vos, C.

2012 Sign-Spatiality in Kata Kolok: how a village sign language of Bali inscribes its signing space. PhD Dissertation. Nijmegen: Max Planck Institute for Psycholinguistics.

Eccarius, P., \& D. Brentari

2007 Symmetry and dominance: A cross-linguistic study of signs and classifier constructions. Lingua 117(7). 1169-1201.

Engberg-Pedersen, E.

1993 Space in Danish Sign Language. The semantics and morphosyntax of the use of space in a visual language. Hamburg: Signum.

Groce, N. E.

1985 Everyone here spoke sign language. Hereditary deafness on Martha's Vineyard. Cambridge, MA: Harvard University Press.

Hendriks, B.

2008 Jordanian Sign Language: Aspects of grammar from a crosslinguistic perspective. Amsterdam: LOT.

Hong, S.-E.

2009 Ein empirische Untersuchung zu Kongruenzverben in der Koreanischen Gebärdensprache [An emperical investigation of agreement verbs in Korean Sign Language] (University of Hamburg, 2008). Sign Language \& Linguistics 12(2). 228-234.

Kakumasu, J.

1968 Urubú Sign Language. International Journal of American Linguistics 34. 275-281.

Kendon, A.

1988 Sign languages of Aboriginal Australia: Cultural, semiotic and communicative perspectives. Cambridge: Cambridge University Press.

Kisch, S.

2008 "Deaf Discourse": The social construction of deafness in a Bedouin community. Medical Anthropology 27(3). 283-313.

Kusters, A.

2010 Deaf Utopias? Reviewing the Sociocultural Literature on the World's “Martha's Vineyard Situations." Journal of Deaf Studies and Deaf Education 15(1). 3-16. 
Lutalo-Kiingi, S.

forthcoming Grammar of Ugandan Sign Language. PhD Dissertation. Preston: University of Central Lancashire.

Marsaja, I. G.

2008 Desa Kolok - A deaf village and its sign language in Bali, Indonesia. Nijmegen: Ishara Press.

Mathur, G., \& C. Rathmann

2006 Variability in verbal agreement forms across four signed languages. In L. Goldstein, D. H. Whalen, \& C. Best (eds.), Laboratory Phonology 8, Vol. 2, 287-313. New York: Mouton de Gruyter.

Nyst, V.

2007 A descriptive analysis of Adamorobe Sign Language (Ghana). Amsterdam: LOT.

Nonaka, A. M.

2004 The forgotten endangered languages: Lessons on the importance of remembering from Thailand's Ban Khor Sign Language. Language in Society 33. 737-767.

Nonaka, A.

2009 Estimating size, scope, and membership of the speech/sign communities of undocumented indigenous/village sign languages: The Ban Khor Case Study. Language \& Communication 29. 210229.

Nonaka, A. M.

2007 Emergence of an Indigenous Sign Language and a Speech/sign Community in Ban Khor, Thailand (Unpublished PhD Dissertation Department of Anthropology, University of California, Los Angeles.

Özyürek, A., I. Zwitserlood \& P. M. Perniss

2010 Locative expressions in signed languages: A view from Turkish Sign Language (TID Linguistics 48(5). 1111-1145.

Padden, C. A.

1988 Interaction of morphology and syntax in American Sign Language. New York: Garland.

Perniss, P. M.

2007 Space and iconicity in German Sign Language (DGS) (PhD dissertation). Max Planck Institute for Psycholinguistics, Nijmegen. 
Sandler, W., I. Meir, C. A. Padden \& M. Aronoff

2005 The emergence of grammar: Systematic structure in a new language. Proceedings of the National Academy of Sciences, 102(7). 2661-2665.

Supalla, T., \& R. Webb

1995 The grammar of International Sign: A new look at pidgin languages. In K. Emmorey and J. Reilly (eds.), Sign, Gesture and Space. Mahwah, NJ: Lawrence Erlbaum.

Tang, G. \& Gu, Y.

2006 Events of motion and causation in Hong Kong Sign Language. Lingua (117)7. 1216-1257.

Washabaugh, W.

1979 Hearing and deaf signers on Providence Island. Sign Language Studies 24. 191-214.

Zeshan, U.

2000 Sign Language in Indo-Pakistan. Amsterdam: John Benjamins.

Zeshan, U.

$2006 \quad$ Interrogative and negative constructions in sign languages. Sign Language Typology Series No. 1. Nijmegen: Ishara Press.

Zeshan, U.

2007 Roots, leaves and branches - The typology of sign languages. In: Quadros, Ronice M ueller de (ed.). Sign Languages: spinning and unraveling the past, present and future. Petropolis: Editoria Arara Azul.

Zeshan, U., C. de Vos, S. Panda, H. Dikyuva \& C. E. Delgado Escobedo

in preparation Number systems in Village Sign Languages: an approach to crossmodal typology

Zeshan, U. \& P. M. Perniss

2008 Possessive and Existential Constructions in Sign Languages, Sign Language Typology Series 2. Nijmegen: Ishara Press.

Zeshan, U. \& Sagara, K. (eds.)

in preparation Semantic fields: Colour, number, and kinship. 


\title{
Colour signs in two indigenous sign languages ${ }^{1}$
}

\author{
Dany Adone, Anastasia Bauer, \\ Keren Cumberbatch and Elaine L. Maypilama
}

\section{Basic Colour Terms in Sign Languages}

Colour has been one of the most discussed semantic domains across disciplines from linguistic anthropology, cognitive psychology, linguistics, and philosophy, to biology and molecular genetics (Berlin \& Kay 1969, Kay \& McDaniel 1978, Palmer 1999, Thompson 1995, Steels and Belpaeme 2005, Mollon et al. 2003 among others). In this paper we investigate colours expressed in two typologically unrelated village sign languages: Yolngu Sign Language (henceforth YSL) in Australia, and Konchri Sain (henceforth KS) in Jamaica. YSL represents a unique case in this volume because it functions as an alternate and a primary sign language in the North East Arnhem Region, NT, Australia (Cooke \& Adone 1994). KS is the indigenous sign language used in the St Elizabeth community in Southern Jamaica, which is characterised by a high degree of bilingualism. The study supports the general findings regarding basic colour terms in sign languages and the data appear to follow the patterns reported for other village sign languages. Additionally, this paper highlights a number of unique structures that have not been reported previously, including the semantic extension of kinship terminology to discuss colours in YSL. By comparing these two unrelated sign languages it becomes clear that each individual village sign language may make substantial contributions to the field of sign language typology.

In their seminal studies on colour naming, Berlin \& Kay (1969) argued that the focal points of colour categories are shared across cultures and languages. They proposed a universal inventory of 11 basic colour terms (BCT) which are defined as being (i) mono-lexemic, (ii) not included in any other term, (iii) not restricted to a narrow class of objects and (iv) psychologically salient. In doubtful cases BCTs are said to have the characteristics of (v) being native, (vi) naming the colour and not the object having that colour, and (vii) having the same distributional potential as the previously established BCTs (p. 6-7). Moreover, Berlin and Kay generalise the hypothesis of 
an evolutionary sequence for the emergence of colour lexicons according to which black and white precede red, red precedes blue, blue precedes purple etc. A number of empirical studies on colour categories in languages that appear not to follow the proposed pattern challenged the universal colour naming theory. More recently, Kay and Regier (2003) further confirmed cross-linguistic similarities in colour naming. Colours can be partitioned into primary, derived and composite. The six primary categories are red, green, blue, yellow, black and white. Six further categories can be derived from these colours (grey, orange, purple, brown, pink, and goluboj which is 'light blue'). While some scholars include goluboj among the derived BCTs given the logic of fuzzy set membership, this issue remains controversial. Corbett and Morgan (1988) and Davies et al (1991) maintained that the two Russian terms sinij 'dark blue' and goluboj 'light blue' fulfil the linguistic criteria for basicness. Recent linguistic studies support the view that there is a single basic blue category but psychophysical studies do not confirm this.

While most of the discussion on colour is based on spoken languages, we note a paucity of in-depth studies on colour in sign languages except for the work of Woodward (1989), Nonaka (2004), Nyst (2007), HagaSchoonhoven, Pfau \& de Boer (2010), Hollman \& Sutrop (2010) and de Vos (2011). Most of the studies describe the colour lexicon of a single sign language such as Ban Khor Sign Language (Nonaka, 2004), Adamorobe Sign Language (Nyst, 2007), Estonian Sign Language (Hollman \& Sutrop, 2010) or Kata Kolok (de Vos, 2011), but there are also a few contrastive studies (Woodward 1989; Haga-Schoonhoven et al. (2010); de Vos 2011). A recurring theme across these studies is the difficulty of applying the defining criteria of BCTs in the context of sign languages (Stokoe 1987; Woodward 1989; Nonaka 2004; Nyst, 2007). Pointing at a body part typically bearing a specific colour (hair for 'black', teeth for 'white', and lips for 'red') is for example one of the widely used strategies for forming colour terms in sign languages. Applying Berlin and Kay's sixth criterion for BCTs, the signs produced by pointing to a body parts or otherwise referring to objects, thus, cannot be considered basic in its strict sense. Stokoe (1987) argues that despite the indexing and the iconic origin of the signs for 'red', 'white', and 'black' in American Sign Language (ASL), they should be regarded as fully lexicalised, especially since the signs for the corresponding objects are distinct from these colour signs. Initialised signs have sometimes also been considered problematic, as they could be considered loans from the spoken language (Woodward 1989). They do however follow the sign language's phonology; moreover, as Hollman \& Sutrop (2010) point out, the additional BCT criteria (v-vii) should only be applied in doubtful cases, i.e. when 
criteria i-iv are not fully satisfied. In this paper, we limit the discussion of the colour lexicons of two unrelated indigenous sign languages to primary, derived and composite colour terms, while touching upon the notion of a $\mathrm{BCT}$ where relevant.

Taking the above considerations into account, the comparative studies on colours in sign languages confirm the broad outlines of the Berlin and Kay findings. Using data from ten different sign languages across seven different sign language families, Woodward (1989) shows that Berlin \& Kay's implicational hierarchy of BCTs holds. Furthermore, Nyst (2007) suggests that when colour signs are grouped according to the morphological process by which they are formed, they generally refer to colours that are adjacent in the BCT hierarchy. Moreover, Haga-Schoonhoven et al. (2010) show that the "basicness" of a colour term is reflected in the phonological markedness of the corresponding colour sign. Specifically, based on a dictionary study of 14 different sign languages, they show that the phonological markedness of colour signs increases as colour terms are less 'basic'.

More recently, de Vos (2011) notes that the colour term systems of five village sign languages (AdaSL, ABSL, BKSL, KK, and PROVISL) show remarkable similarities in that they all have three to four colour terms. As such they are markedly different not only from the spoken languages of the respective wider hearing communities, but also from the urban sign languages documented thus far. Each of these sign languages also allows the use of non-lexical colour descriptions: object naming and pointing to objects in the vicinity. Contrastingly, the primary colour terms of urban sign languages documented thus far appear to align with the spoken languages that surround them. De Vos (2011) hypothesises that the differential lexicalisation patterns between urban and rural sign languages may be explained by social factors. Rural signing communities often constitute dense social networks with lots of face-to-face interaction and well-known conversational partners. For this reason, signers may be well aware of each other's idiosyncrasies and tolerate more lexical variation in expressive forms. An additional factor may be that rural sign languages have not been used in institutionalised settings until recently (but see the sociolinguistic sketches of AVSL and KK in this volume). The histories of urban sign languages are often linked to the establishment of deaf education, and this may accelerate the calibration of their lexicons to the surrounding spoken language and culture.

As aforementioned, this paper discusses the colour terms from two unrelated rural sign languages and brings new data to the table to assess previous generalisations. These two sign languages deviate from typical rural signing communities and thus test hypotheses regarding the impact of social settings 
on sign language colour lexicalisation. The remaining parts of this article are organised as follows: in section 2 we analyse the findings from YSL, and in section 3 we focus on KS. In section 4 we present some preliminary conclusions and remaining issues.

\section{Colour Terms in Yolngu Sign Language (YSL)}

\subsection{Sociolinguistic Settings of YSL}

According to the latest estimates, there are approximately 5000 Yolngu $^{2}$ Aboriginal people living in former mission settlements along the far northeastern coast of Arnhemland, in Northern Territory, Australia (s. Figure 1) (Christie and Greatorex, 2004). The number of people living in Yolngu communities vary between 500 and 2000. One-third of Yolngu people reside in family groups on traditional ancestral lands in the so-called homelands. Some Yolngu also live in Darwin, the capital city of the Northern Territory.

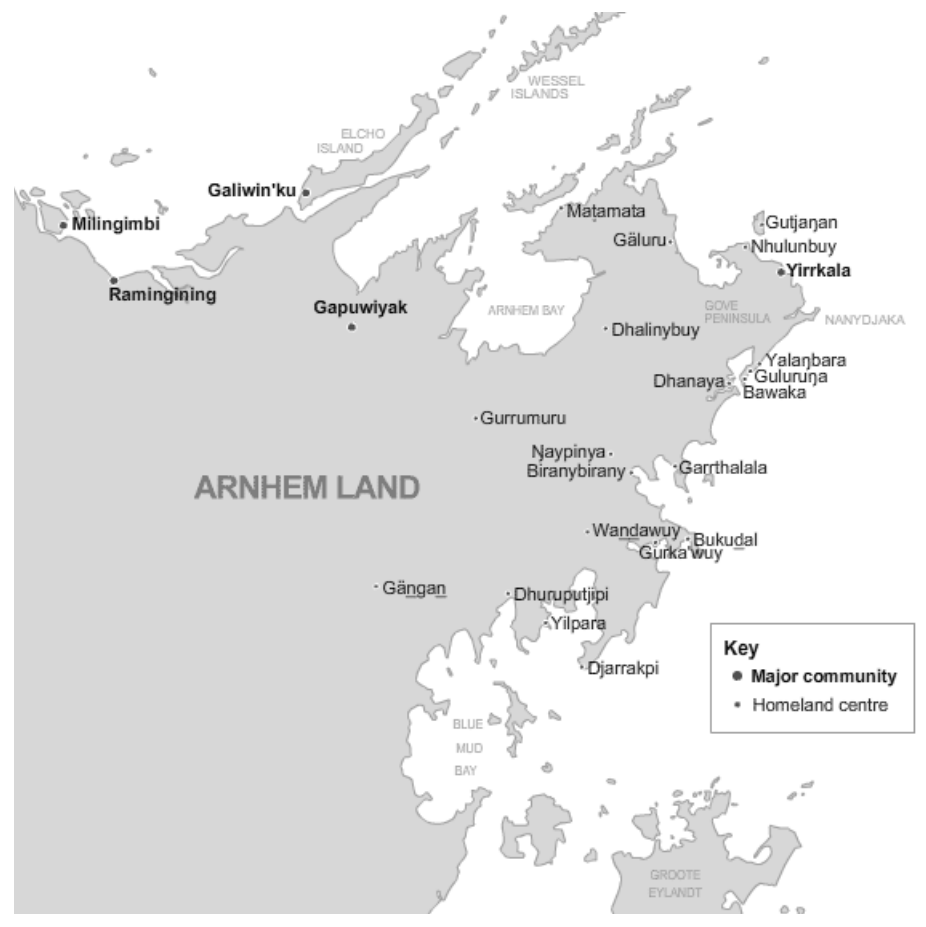

Figure 1. Yolngu area in North East Arnhem Region (Map courtesy of the Australian National University) ${ }^{3}$ 
In 2009 and 2010 the data were collected in three places. The first is Darwin, the capital city of the Northern Territory, where many Yolngu work as interpreters. The second place is a remote homeland, Mapuru, and the third is a large settlement Galiwin'ku on the Elcho Island. Galiwin'ku is one of the largest Aboriginal settlements in Australia established as a Methodist mission in 1942 on Elcho Island (Devlin, 1986) (see Figure 1). It is populated by approximately 2,000 Yolngu and a number of non-Indigenous administrators, community workers and teachers. The exact number of residents living in Galiwin'ku is unknown. Population varies during the seasons, with many Yolngu migrating to the homelands during the wet season. Mapuru is situated on the mainland nearly $600 \mathrm{~km}$ away from Darwin (see Figure 1) and was established by two families in the 1960 s. Today there are approximately 70 people (Greatorex p.c). During the dry season there are Deaf people visiting their relatives in Mapuru.

The term 'Yolngu' generally refers to a socio-cultural group of people and their language varieties. All members of this group are assigned to a category based on the one to which their fathers or mothers belong. These categories are 'moieties', 'clans', 'sections', 'subsections' and gurrutu, the complex, extended kinship system. The entire Yolngu universe is divided into two mutually exclusive but complementary groups or moieties, termed Yirritja and Dhuwa. The world is thus divided in half and a person belongs to the same moiety as their father.

Yolngu are multilingual and have at least some passive knowledge of some of the other Yolngu languages. Children normally first acquire their father's language and later their mother's. In Galiwin'ku for example, one Dhuwal dialect, Djambarrpuyyu, has evolved as a lingua franca (Devlin, 1986; Wilkinson, 1991). Besides Djambarrpuyngu, Yolngu people speak Gupapuyngu, Gumatj, and Djapu, together with Kriol, and Aboriginal English. Besides spoken languages, every child also acquires signs from birth.

It is a well-established fact that sign language is widespread in Aboriginal communities. Both hearing and deaf people use it to communicate (Kendon, 1988; Adone, 2001; O’Reilly, 2006; Green, Woods, \& Foley, 2011). Kendon (1988) classified these sign languages as alternate sign languages because they were developed for cultural reasons and are used as alternatives to speech. Other examples of alternate sign languages are Sawmill Sign Language and Plains Indian Sign Language (Kendon, 1988; Pfau, in press). As compared to North Central Desert area sign languages, described by Kendon (1988), YSL is not a signed version of any spoken Yolngu language and, moreover, displays little relationship to the surrounding spoken languages (Cooke \& Adone, 1994; Bauer, in prep.). YSL also serves as a 
primary means of communication for a number of deaf members in Yolngu communities. Based on the information Adone gathered in July 2012, there are roughly 40-50 deaf people and a few hundred with hearing impairments throughout Arnhem Land. As for the settlement in Galiwin'ku, there are seven members of the community who are deaf. In this respect the figures here have remained constant since 1994. YSL functions as both an alternate and primary sign language, used in daily activities such as interaction at a distance, when speaking causes too much effort, exchanges between deaf and hearing people, and communication when hunting. It may also be used under various cultural circumstances, e.g. when silence is required as in ceremonies, or in the "proximity of sacred objects when silence is culturally requested" (Cooke \& Adone, 1994).

\subsection{Data Collection}

Data on YSL was first collected by Cooke and Adone in the early 90s, followed by several field trips in the late 90s. In 2008 Adone collected further data with the help of sociolinguistic questionnaires. Bauer collected spontaneous and elicited data based inter alia on the colour questionnaire designed by Sagara, de Vos and Zeshan (iSLanDS, Preston, UK) in 2009 and 2010. Data was gathered from nine hearing and three deaf signers from Mapuru, Galiwin'ku and Gove (s. Figure 1). Table 1 provides an overview of the participants' characteristics, the locations where data was collected, date of fieldwork, and signers' age, gender and hearing status.

Table 1. Participants' characteristics

\begin{tabular}{cccccc}
\hline Location & Year & Subject & Gender & Age & Deaf/Hearing \\
\hline \multirow{2}{*}{ Darwin } & \multirow{2}{*}{2009} & HG & female & late $50 \mathrm{~s}$ & hearing \\
& & RB & female & early $30 \mathrm{~s}$ & hearing \\
\multirow{2}{*}{ Mapuru } & 2010 & JD & male & early $40 \mathrm{~s}$ & hearing \\
& & LB & male & late $60 \mathrm{~s}$ & hearing
\end{tabular}




\begin{tabular}{|c|c|c|c|c|c|}
\hline \multirow{7}{*}{ Galiwin'ku } & \multirow{7}{*}{2010} & EB & male & late $20 \mathrm{~s}$ & hearing \\
\hline & & MD & male & late $20 \mathrm{~s}$ & hearing \\
\hline & & ER & female & late $30 \mathrm{~s}$ & $\begin{array}{l}\text { became deaf } \\
\text { postlingually }^{4}\end{array}$ \\
\hline & & $\mathrm{EG}$ & female & late $50 \mathrm{~s}$ & hearing \\
\hline & & W & female & early $40 \mathrm{~s}$ & deaf \\
\hline & & $\mathrm{DM}$ & male & early $60 \mathrm{~s}$ & hearing \\
\hline & & MG & male & 10 y. o. & deaf \\
\hline
\end{tabular}

The signers were filmed with a video camera as they provided descriptions of different colours. The annotations were made using ELAN digital annotation software (http://www.lat-mpi.eu/tools/elan/). For the research on colour vocabulary, two tasks were performed: a simple colour chip naming task and translation from English, which was only possible with hearing signers competent in both English and YSL. The naming task was explored using 17 colour chips. This set consisted of blues, reds, greens, and yellows, with three degrees of brightness, as well as black, white, purple and brown. Altogether eight hours of unedited digital video footage was collected, and this was edited down to six hours, 18 minutes and 16 seconds of usable language production.

\subsection{Colour terms in surrounding spoken languages and culture}

Before we tackle the question of colour in YSL, it is useful to look at colours in the Yolngu languages in the surrounding area. Yolngu refer to their spoken languages collectively as Yolngu Matha (lit. tongue, speech) (cf. also Zorc, 1986). The majority of the study's informants used Djambarrpuyyu, a variety of the Dhuwal-Dhuwala dialect group.

In Djambarrpuynu there is no generic term for 'colour'. However, the term miny'tji is used to cover "colour, design, paint or colouring material as clay or ochre" (Zorc 1986) and there is also an YSL sign that expresses this concept. Nowadays the English term colour has been incorporated into the everyday interaction. Adults as well as children use the English term on a regular basis to refer to any items of colour such plastic tupperware, plastic bags and all accessories of modern life. 
The following colour terms exist in Djambarrpuyyu: watharr 'white' or 'light coloured'; mol 'black' or 'dark coloured'; miku 'red'; buthalak 'light yellow'; gangul 'dark yellow'; milkuminy 'blue/green'; ratjpa 'reddish brown'; arrani 'brownish'; murngun 'pink'; gulay-gulay 'blood red' (Zorc, 1986; Wilkinson, 1991). Further colour terms are also reported such as lirrgi 'black charcoal' or dunguimirr 'soil black with humus' (Davis, 1982). In contrast to the system of colour encoding in English, which is based on the differentiation of hues, Yolngu people seem to encode colour terms by the brightness contrast. The colours are thus considered in terms of their degree of saturation compared to black and white. For example, yellow is not as light as white, and green is not as dark as black. Colour terms mol 'black' and wathar 'white' are apparently the first colour terms to be acquired by the Yolngu children (Davis 1982).

\subsection{Colour Signs in YSL}

In parallel to the concept miny'tji in Djambarrpuyyu, YSL has a sign that does not refer to 'colour' exclusively, and is rather regarded as the overall design of an object. ${ }^{5}$ The sign is made with a 6-handshape by placing the thumb at the side of the nose as shown in Figure 3. It is also similar, as examples (1)-(2) show. We find that this sign is used with spoken components, the so-called "mouthings" kala or minyt'ji. Kala is borrowed from the English word 'colour' by Yolngu speakers who are more or less proficient in English. Kala, as seen in Aboriginal English, is the approximate translation of 'colour' and a typical example of Australian languages borrowing from English for new concepts.

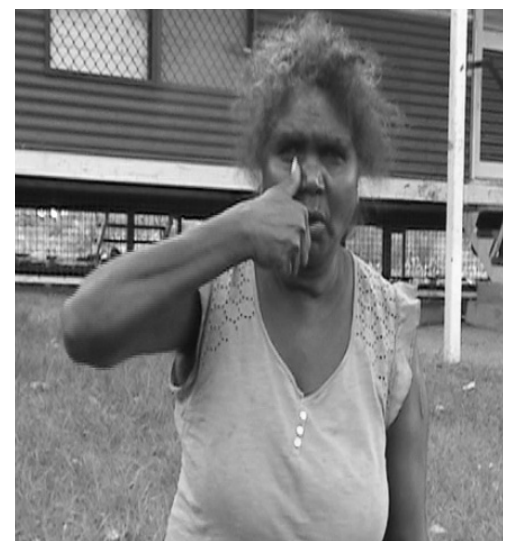

Figure 2. MINY'TJI 'colour/design' in YSL 
Here are two examples for YSL sign MINY'TJI 'colour/design'. In (1) the sign MINY'TJI refers to the size of object and in (2) to the colour. Notice that whenever the sign MINY'TJI means 'colour', the meaning is specified by the mouthing. No mouthing is found to be accompanying the sign with the meaning 'size of the object' as shown in (1).

1) GUYA

fish

"It is a big fish."
MINY'TJI

colour/design
YINDI

big

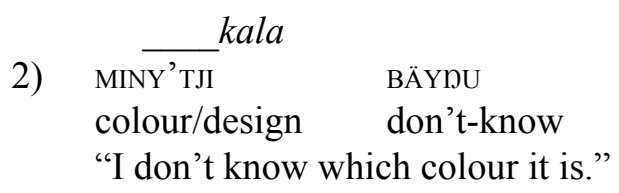

YSL has two signs referring to light and dark skin complexions. The sign MOL 'black/dark' is made by pointing to the opposite upper chest or arm of the signer with either 1 or $\mathrm{B}^{6}$ handshapes (Figure 4) and is used to refer to any Aboriginal or other person of dark complexion. The sign for WATHARR 'white/light' is made by quickly rubbing the arm of the signer with the B-handshape (s. Figure 4). This sign is used to refer to a white person, also known as 'balanda'. Both signs can also be used to refer to the colours 'black' and 'white' more generally.

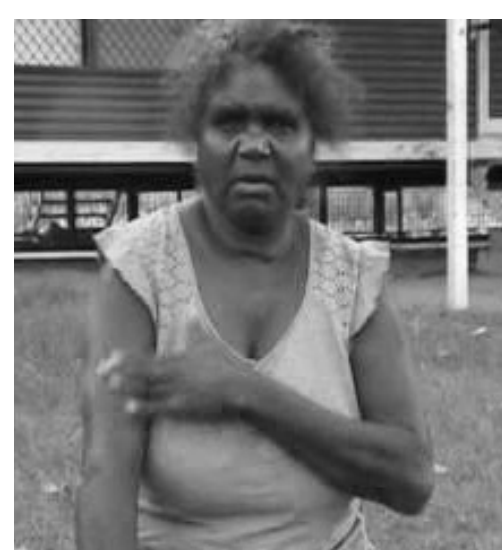

WATHARR white/light

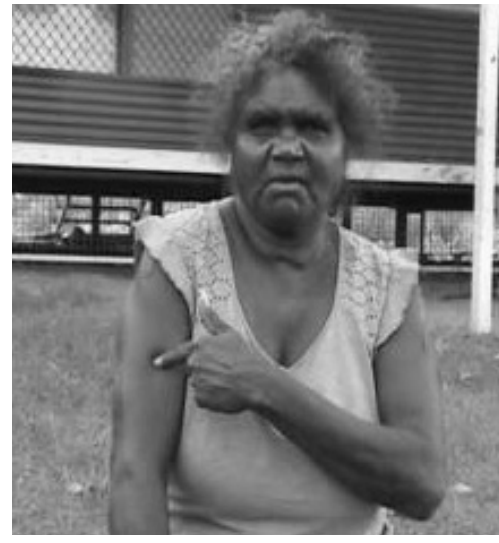

MOL

black/dark

Figure 3: Two colour signs in YSL 
When asked to express the colour WATHARR 'white/light', some signers also pointed with 1-handshape at their own teeth (s. Figure 5). This sign was however very infrequent in the data.

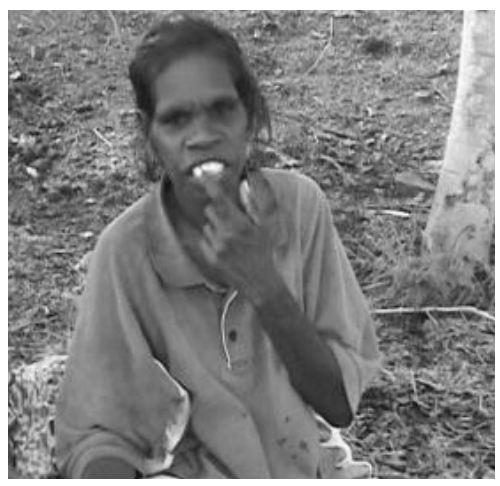

WHITE

Figure 4. Variation sign for watharr 'white/light' in YSL

In earlier data collection (Adone 1994 and subsequent work) we find further lexical signs for primary colours such as black, red and yellow. These colours are commonly used to paint faces in ceremonies in Galiwin'ku. The colour 'red' is expressed by the reduplication of the sign MINY'TJI 'colour/design' shown in Figure 3 above. The sign BUTHALAK 'yellow' is articulated by moving a 1- or 2-handshape under the eyes from the left to the right side of the face. The sign for 'white' is signed with the 4-handshape or with the 2-handshape moving from the top of the forehead to the middle of the head. This sign is used in ceremonial contexts referring to the white colour of body painting. The sign referred to here as MOL-2 is a compound of MOL 'black' and GUNDA 'rock' (Figure 6) which indicates 'black as a rock'.

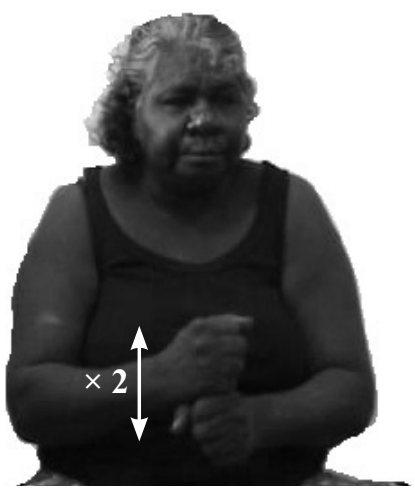

Figure 5. The YSL sign gunda 'rock' 
These signs are used in ceremonies as well as in daily life to express colours. Most of the older people and elders use these colours regularly while the younger people use them less.

There is also another lexical compound sign for gulay-gulay meaning 'blood red' which consists of GAPU^GURRKURR^MINY'TJI. The sign GAPU 'water' is expressed with the O-handshape as shown in Figure 7. The second part of the compound, GURRKURR 'run-through-veins', is formed by repeated movements along the arm indicating blood in the blood vessels with the 4-handshape.

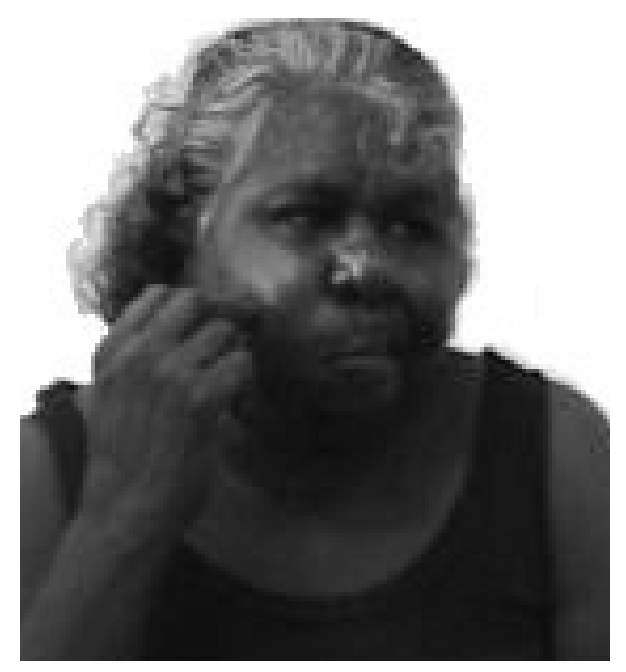

Figure 6. The YSL sign gapu 'water'

Drawing on the earlier data gathered (Adone 1999, 2002) and the 2009-2010 data, it becomes clear that YSL has at least six lexical signs and one lexical compound in the domain of colour. There are two variants for 'black': one primarily referring to the skin colour MOL 'black/dark', and one used in ceremonies MOL-1 'black'. Two variants for 'white' are WATHARR 'white/ light', also mainly referring to skin colour, and the colour sign WATHARR-1 'white' in ceremonies. Additionally, 'white' is occasionally referred to by pointing to the teeth. A reduplication of the lexical sign meaning 'colour/ design' (MINY'TJI^ MINY'TJI), is taken to indicate the colour 'red'. Table 2 below presents an overview of YSL and Djambarrpuynu colour terms. 
Table 2. Colour terms in Djambarrpuyyu and in Yolngu Sign Language

\begin{tabular}{|c|c|}
\hline $\begin{array}{c}\text { Djambarrpuyju } \\
\text { (Zorc, 1986; Wilkinson, 1991; Davis, 1982) }\end{array}$ & Yolngu Sign Language \\
\hline miny'tji 'colour/design' & MINY'TJI 'colour/design' \\
\hline mol 'black, dark coloured' & MOL 'dark skin', 'black' \\
\hline dunguimirr 'soil black with humus' & MOL-1 'black (dye)' \\
\hline lirrgi 'black charcoal' & $\mathrm{MOL}^{\wedge} \mathrm{GUNDA}$ 'rock black' \\
\hline \multirow{3}{*}{ watharr 'white, light coloured' } & WATHARR 'light skin, white, colour' \\
\hline & WATHARR-1 'white' \\
\hline & $\begin{array}{l}\text { WATHARR-2 'white' (pointing to } \\
\text { teeth; infrequent) }\end{array}$ \\
\hline miku 'red' & MINY’TJI^ MINY’TJI 'red' \\
\hline gulay-gulay 'blood red' & $\begin{array}{l}\mathrm{GAPU}^{\wedge} \mathrm{GURRKURR}^{\wedge} \mathrm{MINY} \text { 'TJI ‘blood } \\
\text { red' }\end{array}$ \\
\hline buthalak 'light yellow' & BUTHALAK 'yellow' \\
\hline \multicolumn{2}{|l|}{ gangul 'dark yellow' } \\
\hline \multicolumn{2}{|l|}{ milkuminy 'blue/green' } \\
\hline \multicolumn{2}{|l|}{ ratjpa 'reddish brown' } \\
\hline \multicolumn{2}{|l|}{ arrani 'brownish' } \\
\hline murngun 'pink' & \\
\hline
\end{tabular}

The data above indicate that YSL appears to have fewer lexicalised colours signs than Djambarrpuyyu. It is however important to note that YSL's colour signs are subject to some forms of modification such as facial expressions which are combined with other signs sequentially or simultaneously to express additional colours as well as nuances. Although facial expressions are variable as they depend on several factors such as the presence of certain people and the signer's kin relationships to his or her interlocutors, we note that features such as squinting and pouting are combined with signs to mark the difference between light and dark shades (Adone, 1999; Adone, 2002). Another interesting point to mention is the use of hedging, i.e. referring to a non-typical colour such as 'bluish' and 'yellowish'. Although hedging is seen in most spoken Yolngu languages, it seems that it is less frequently used in YSL (Adone, 1999). Besides a few examples of hedging in spontaneous speech, we did not find this phenomenon in elicited data. More data 
will be needed to identify further differences between colour descriptions in Djambarrpuyyu and YSL.

\subsection{Non-lexical colour descriptions in YSL}

Two additional observations are worth mentioning here. First, the use of pointing to refer to colours, and second, the use of kin terms to refer to colours. When signers cannot relate an equivalent for a colour term, they point to an object sharing the same colour, e.g. to the ocean for 'blue', or to a piece of clothing for 'green', to indicate the colour in question. This strategy is very frequently used by signers to refer to non-lexicalised colours (see also de Vos, 2011). Pointing to an object that has the colour they are looking for functions as a form of colour description. A similar strategy is also witnessed in Djambarrpuyyu. When talking about a colour foreign to the language, speakers might name an object in the vicinity of the speaker with the designated colour. Rocks, tree bark, pandanus leaves or bush fruits often serve as a source for analogy (see also Wilkinson, 1991). Signers may also point to animals as in (3). In a discussion on what is regarded as 'grey', a deaf Yolngu signed MINY'TJI 'colour/design' and pointed to a blue tongue lizard, which is a bluish/greenish grey. Note that the sign for 'colour' does not have a fixed order in the sentence:

3)

MINY'TJI INDEX
colour/design pointing to the blue tongue lizard
'colour grey with bluish and greenish'

This grey was distinguished from the dark grey of (cumulonimbus) ${ }^{7}$ clouds:

4)
MINY'TJI
INDEX
colour/design
pointing to the cloud
'grey of a cumulonimbus cloud'

To make the difference clear between these two shades of grey signers use mouthings of the spoken Djambarrpuynu words mirrthirr 'very' or marrganga 'little' as well as facial expressions to express shadings.

Some Yolngu signers also use the gurrutu paradigm, the extended Yolngu kinship system to refer to colours. As already noted above, the entire Yolngu universe is divided into two different, mutually exclusive but complementary moieties Yirritja and Dhuwa, and every single thing in the Yolngu world thus belongs to one of the moieties: the land, the people, the languages, the 
animals and all the natural phenomena such as water, sand and plants. This is also reflected in the Yolngu paintings, which are recognised as property of one moiety or clan via particular ancestral designs, representations of animals, geometric background patterns, cross-hatching and the dominance of a certain base colour (usually black, red or yellow) (Morphy 1989). In addition to using a pointing strategy to refer to colours other than black and white, two participants used a kinship reference strategy to name several colours in the elicitation task during the fieldwork in 2010. The following procedure was applied during the elicitation session: one YSL signer was asked to view the colour chips sheet and present a sign for each colour one by one. Another signer who viewed the signed production was then asked to translate it into Djambarrpuyyu. When seeing the red colour, the first signer gave the following answer:

$\begin{array}{lll}\text { INDEX }_{a} & \text { INDEX }_{b} & \text { DÄNDI } \\ \text { pointing to the red colour } & 1 S G & \text { mother } \\ \text { "It is my mother's colour." } & & \end{array}$

The other signer translated this as 'red' and explained that there is a connection between the red colour and his mother's moiety. When seeing the yellow colour, the signer referred to his father. Given that marriage is exogamous in Yolngu society, children always belong to a different moiety and clan from their mothers. Thus the two signers, by using the kinship reference strategy, showed an association of the red colour with the Dhuwa moiety and yellow colour with the Yirritja moiety. However, using kinship to relate colours is not common. While every single member knows the colours associated with the Dhuwa and Yirritja moieties, these are not normally referred to publicly. In this case the link between kinship and colour was made to explain cultural practices to the researcher. The interrelationship of colour domain and social organisation has also been observed for other Australian groups (Hill 2011).

\subsection{Summary}

In terms of primary colours, YSL has six signs with two variants for 'black', two variants for 'white', and lexical expressions for 'red' and 'yellow'. The colour signs MOL-1 'black', WATHARR-1 'white', MIKU 'red' and BUTHALAK 'yellow' are used in ceremonies. WATHARR 'white/light' refers to light, bright colours and MOL 'black/dark' refers to dark, dull and black colours. These terms are used to refer to white and black skin, which 
by extension applies to the distinction between white and Aboriginal people. In YSL the distinction between 'light' and 'dark' (WATHARR vs. MOL) thus seems to form the basis for the distinction between black and white. The term MINY'TJI is one term used to refer to colour, design and shape, all of which are important components in Aboriginal culture. These findings confirm to some extent what Hargrave (1982), Wierzbicka (2006) and others have pointed out for the spoken languages of the surrounding area, namely that these languages might not have a specific term for 'colour'.

Groups and clans identify with colours, designs and shapes. The Dhuwa moiety which includes Djambarrpuyngu and YSL uses red ochre (miku), heamatite (ratjpa), and yellow ochre (buthalak) as well as white clay in their ceremonies and paintings. When an elder member of the Liyagawumirr group talked about his painting to Adone and colleagues in 1992 he explained that the Dhuwa groups used certain designs and colours in their paintings such as combinations of straight parallel lines and circles. Each pattern is associated with a particular Wangarr 'ancestor' and thus links the group to other groups through the past, present and future. This interrelation between visual perceptual properties and the kinship system in Aboriginal cultures allows for the kinship reference strategy in YSL, which is has not been attested in any other urban or rural sign language (Maypilama and Adone 2012).

Hargrave (1982) argued that perhaps the nomadic traditional lifestyle of Aboriginal people could have been the reason why they did not need words to describe the different colours in their surroundings. The Yolngu people as their neighbours have used several pigments like white pipe clay, charcoal, red ochre, yellow ochre, and haematite for bark and body paintings, ceremonies, etc, for a long time. These pigments must have been the only ones used in traditional times, as they have moiety affiliations. In this context it is important to note that haematite used by the Yolngu from Elcho Island was a special kind of ochre that was not found elsewhere and was thus traded to the surrounding groups in the area. The fact that certain colours and designs are central to the culture for establishing links between past and present, and among ceremonies, sacred objects and ancestors of the different groups, is a strong argument for the existence of a wide range of colours in the Yolngu languages.

For artificial objects and unfamiliar colours, signers pointed to physical objects in their proximity, a strategy commonly used in other sign languages without lexicalisation of signs (Washabaugh, Woodward, \& DeSantis 1978; Nonaka 2004; Nyst 2007; de Vos 2011). Overall, it is clear that the Yolngu spoken languages have a wide array of colours that are important in their 
culture, and it is therefore surprising that YSL has fewer terms. There might be several reasons why certain colours are not yet lexicalised in YSL. One of them is perhaps the auxiliary nature of this language for the majority of its signers. As mentioned in section 2, the Yolngu also consider certain colours as 'sacred', and perhaps for this reason they feature in both signed and spoken languages. Other colours which are less culturally relevant, such as 'grey' or 'purple', can be described by pointing.

Finally, it should be noted that the sign MINY'TJI 'colour/design' (see Figure 3) is often accompanied by mouthing of the word kala. This borrowing taken together with the frequent use of other English colour terms wurintj 'orange', bilu, 'blue' and guriny guriny 'green' is first-hand evidence for the cultural and conceptual impact English has on Aboriginal languages. It is therefore possible that the system may be in transition under the influence of a more extensive colour term system.

\section{Colour Terms in Konchri Sain (KS)}

\subsection{Sociolinguistic Settings of KS}

Konchri Sain (KS) is an indigenous sign language native to rural Southeastern Jamaica in the Northern Caribbean. The map below (Figure 8) shows Top Hill where KS originated. Additionally, the towns and villages south of Main Road Class 1 (the red line) are surrounding communities in which KS is used. Patwa (Jamaican Creole) and English are the two main spoken languages used in Jamaica. These are used in the area shown in Figure 8.

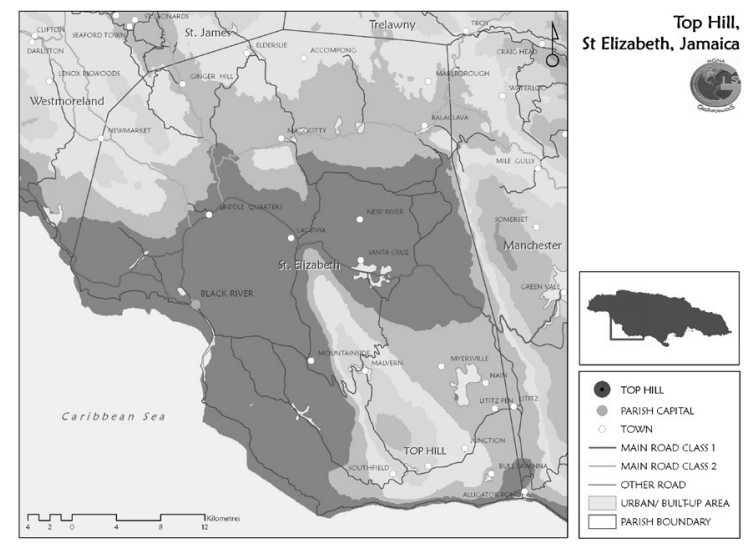

Figure 7. Top Hill, Jamaica (Map courtesy of the Jamaican Language Unit) 
KS is now an endangered language that coexists alongside two other signed communication systems which carry far more prestige in the community. These other sign systems, Jamaican Sign Language and Signed English, are used in urban areas and in deaf education. Jamaican Sign Language is evolving out of American Sign Language which was introduced by Southern Baptist missionaries. The missionaries established the first schools for the Deaf and used American Sign Language and Signed English as languages of instruction. The schools are now under local management and Signed English is still a medium of instruction. Without constant input from American Sign Language users, the signed communication system began to change in syntax and lexicon. This evolving signed system is Jamaican Sign Language. Most schools have acknowledged Jamaican Sign Language not American Sign Language as the native sign language of its pupils and made the shift to its use in the classroom.

Despite research into KS structure being in the initial stages, the research has had the positive effect of reversing negative language attitudes and reviving its use. One deaf informant commented that after using KS during data collection, she started teaching it to her grandchildren. This is a welcome change since there is a high incidence of deafness within Top Hill and the immediately surrounding communities. If transmission of this language is sustained, it may result in the younger generation using KS for daily interaction and their seeing it as just as valid a language as JSL and Patwa. Increased prestige for KS can secure its status as a heritage language in its multilingual environment. Perhaps, a reversion to yesteryear may be seen where all persons in the area, both hearing and Deaf, used KS. Nowadays, the hearing people who sign use mainly JSL to communicate with the Deaf. KS is only used with persons who are monolingual in KS.

\subsection{Data collection}

Cumberbatch collected KS data from 2009 to 2011 using various elicitation instruments including the colour questionnaire designed by Sagara, de Vos and Zeshan (iSLanDS, Preston, UK). The researcher used JSL when eliciting data and KS when checking her interpretation of the data. Data was collected from several deaf persons in the Top Hill community at an informal gathering and several times after church. There were three primary informants for the colour questionnaires. Two of them, teachers, are fluent users of Konchri Sain, Jamaican Sign Language and Signed English. The other, a farmer, is a Konchri Sain user with limited competence in JSL. As was done 
for YSL data management, the interactions were digitally filmed and then annotated using ELAN video annotation software.

\subsection{Colour Terms in Surrounding Signed and Spoken Languages}

KS colour terms do not seem to be influenced in any way by the spoken languages, Patwa (Jamaican Creole) and English, that are also used in the community. Similar to YSL, KS has its own colour signs, and pointing at objects is also used as a strategy to express colours. The colour terms of KS, Jamaican Sign Language (JSL), Jamaican Creole and English show dissimilarities. The two spoken languages, Jamaican Creole and English, have the same colour terms. Patwa and English have colour terms that span the visible spectrum of colour. Jamaican Sign Language has fewer colour terms than the spoken languages. In addition, JSL has conceptual categories of human colour as opposed to those of nonhuman colour. This distinction does not exist in the surrounding spoken languages. The JSL signs that denote human skin complexion are translated into English as BLACK.Human, wHITE.Human and BROWN.Human. JSL also has different signs that represent the colours black, white and brown as properties of nonhuman entities. The distinction between colour terms referring to human and nonhuman entities does not exist in KS. Evidence for this was found in the sign name of a lady which was FAT-WHITE. The WHITE in her name sign which referred to her pale complexion was the same WHITE used to describe nonhuman entities. Patwa and English have the same colour terms for humans and nonhumans but ASL does not. Therefore, it can be safely assumed that JSL took this from ASL. The influence of JSL, the other sign language in the environment, on KS was seen in another way. There were cases where bilingual KS and JSL users also produced the JSL signs for these colours. An informant produced the JSL sign PURPLE as well as the KS sign GRAPE when referring to the colour 'purple'.

KS seems to have the least number of colour terms of the four languages of this community. Some variation in colour terms has been observed but the most likely reason for this seems to be the instability typical of a critically endangered language rather than a lack of conceptual categories.

\subsection{Primary Colour Signs in KS}

This section looks at colour in Konchri Sain. KS has a generic sign for the concept 'colour' and employs more than one means to express colour terms. 
The KS sign for COLOUR is a left to right movement of the 1-hand in front of the torso (see Figure 9). The sign has slight lip rounding. It occurs as a two-handed sign as well as a one-handed sign. However, the two-handed form only occurs to show pluralisation. Therefore, it can be argued that the 1-handed form of the sign is the sign COLOUR while the two-handed form is a total reduplication that functions as a plural marker.

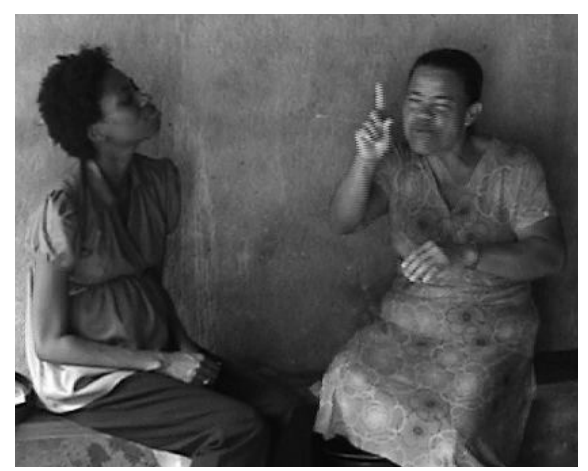

Figure 8. COLOUR - 1-handed form

The six primary colours - black, white, red, yellow, green and blue - are expressed in KS. In examining the data, two forms of BLACK were observed. BLACK is produced on the cheek by rotating the A-hand $90^{\circ}$ downward. A far less common variant, seen thrice in the data, uses the clawed hand moving downwards from the cheek past the chin. The more widely distributed variant can be seen below in Figure 10. Mouthing of the English word, black, appears to be for the benefit of the researcher as it is not seen when KS users are communicating among themselves. WHITE is made by an arc motion of the flat hand (B across the face as seen in Figure 11. This sign is sometimes done with the 5-hand.

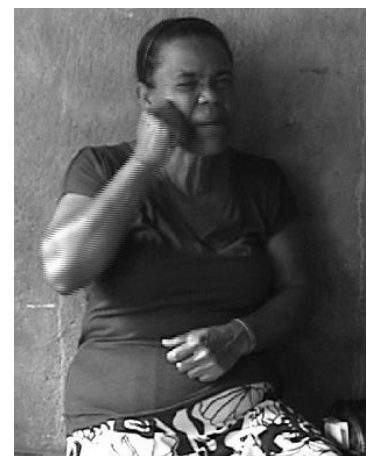

Figure 9. BLACK

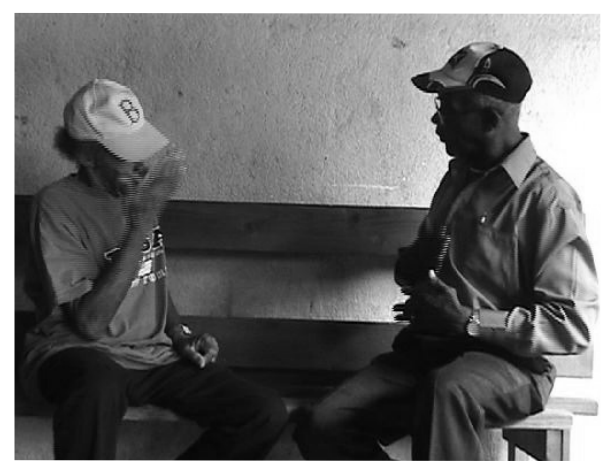

Figure 10. white 
The generic sign, COLOUR, occurs in conventionalised combinations with mouthing. This is seen in YELLOW, BLUE and RED where parts of the corresponding English/Patwa words are produced. The words meaning 'yellow', 'blue' and 'red' in English and Patwa have the same phonological forms. Therefore, the mouthings could have been derived from either spoken language. YELLOW is produced with the tongue between the teeth. This sign is labelled as YELLOW although it is also used when referring to colours translated into English as orange. This is because the associated mouthing matches the mouthing found in English yellow. BLUE has exaggerated lip rounding. This correlates with lip rounding in English blue. RED has exaggerated lip pouting. English red has slight lip pouting. These KS signs are illustrated in Figures 12-14. Adamarobe Sign Language (AdaSL) has a very similar system with the index finger and partial mouthings from the surrounding spoken language being used as components of lexical items that represent colours (Nyst 2007). However, AdaSL employs this strategy for BLACK, WHITE and RED while KS does it for YELLOW, BLUE and RED. As such, the KS data confirm Nyst's (2007: 92-93) hypothesis that colour signs that are formed in the same way generally refer to colours that are adjacent in the colour hierarchy. The use of the mouthings concurrently with COLOUR was consistently seen throughout the data making it clear that these are established signs in KS. The expression of green in KS is discussed in $\S 3.5$.

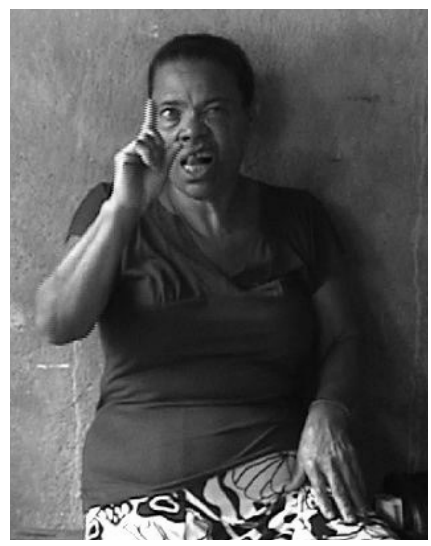

Figure 11. YELLOW

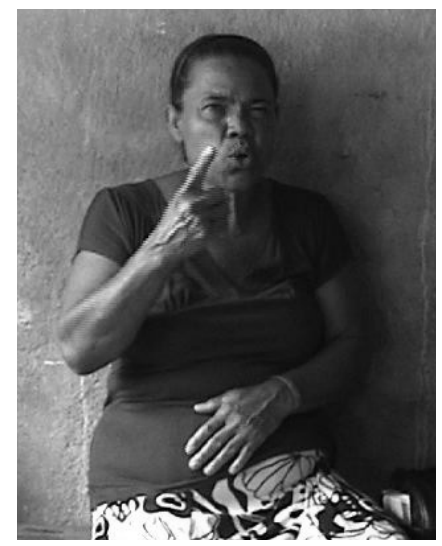

Figure 12. BLUE 


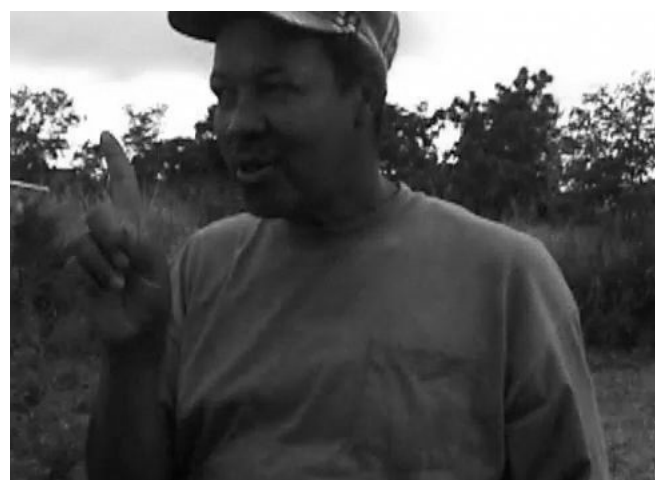

Figure 13. RED

\subsection{Colours and Entities in the Surroundings in KS}

Clear semantic motivation is also evident in the signs for some colour categories. Entities regularly encountered in daily life are used to refer to some colours. Context will indicate if the signer is referring to the entity itself or the colour property of that entity.

'Red' can be expressed in three ways. The first, as aforementioned and illustrated in Figure 14, is to produce the sign COLOUR with the mouthing. The second and the third involve the use of other KS words, namely KILL and BLOOD. These are shown below. The practice of killing animals for their meat is the cultural activity that may have motivated these forms of RED. Each signer produced all variants to refer to the same shades of red. Future research has been planned to investigate possible phonemic, semantic or other motivations for each variant.

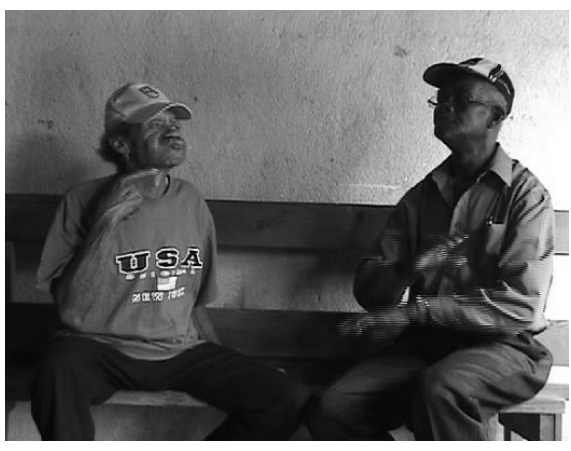

Figure 14. KILL

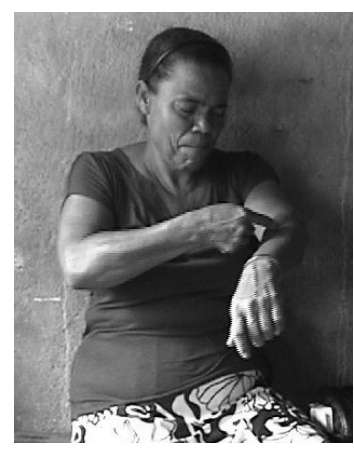

FIGURE 15. BLOOD 
Being farmers, members of the Top Hill community spend many of their waking hours outdoors. Items in their physical environment are also used in KS for 'yellow' 'blue', and 'green'. 'Yellow' is sometimes expressed by signing SUN. There is a direct link between the colour of the sun and its use to represent the colour 'yellow'. The KS sign TREE is also used to refer to green. SKY is used for 'blue'. When these nouns are signed to refer to colours, the generic sign COLOUR sometimes occurs before or after the noun.

The hues of the people themselves are also reflected in the colour terms of $\mathrm{KS}$. BROWN is another such KS colour sign. KS users are of negro descent so the skin on the back of their hands is varying shades of brown. The sign is semantically related to the colour of the dorsum of the hand. Figure 17 shows two forms of the sign BROWN. Likewise, PINK is expressed by pointing to the palmar surface of the hand.

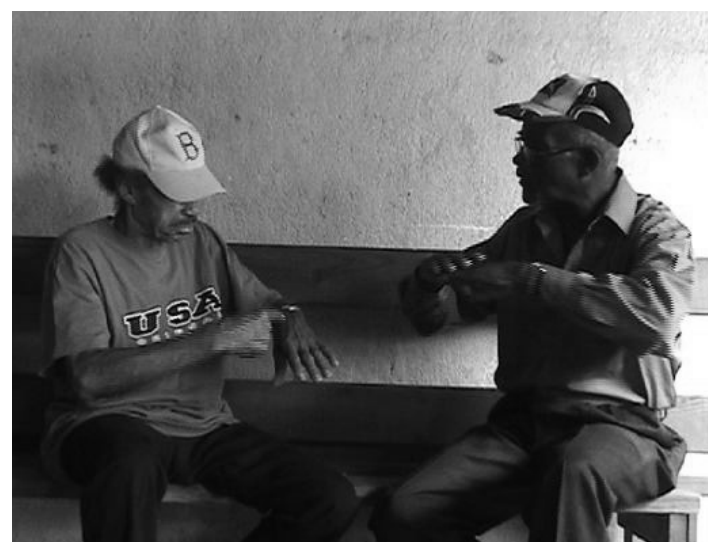

FIGURE 16. TWO FORMS OF BROWN

Fruits were the semantic motivation for some colour terms like those meaning 'orange' and 'purple'. For these, the signs for the fruits orange and grape, respectively, were used to represent the colours. The skin of the variety of the fruit in this area is not orange in colour but a mixture of yellow, brown and green while the pulp is orange. The KS sign is ORANGE-CUT which clearly refers to the colour of the fruit pulp. Similarly, the skin and pulp of grapes first introduced to Jamaica are purple in colour. It should be noted that although green, black and red grapes are also now available in Jamaica, purple is still the colour associated with the fruit. 


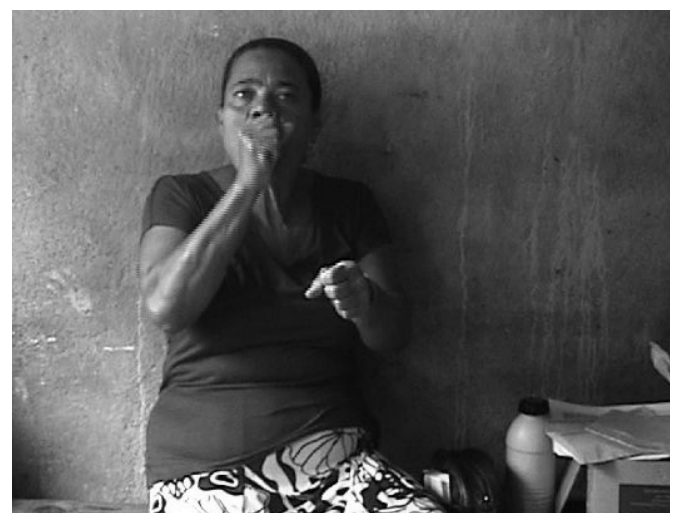

FIGURE 17. ORANGE-CUT

Non-lexical strategies are used when referring to a colour that has no colour term in KS. Instead of producing a sign, pointing to an object of that colour is employed. However, this can be argued to be lexical if the pointing is viewed as pronominal. An example of this is a colour like burgundy, a very dark red, where the signer points to a piece of cloth that is burgundy. There is a formational level of conventionalisation in the handshape used. A 1-hand is used. This 1- hand often occurs with the signer looking around for a matching object.

\subsection{Colour Constructions in KS}

Other signs can occur immediately alongside KS colour signs to give more information about the colour properties of objects. In most instances, these modifying words were found after the colour term.

Two examples are given below.

9) PINK STRONG

pink bright

'bright pink'

10) Lips: rounded

$\begin{array}{ll}\text { COLOUR } & \text { BRIGHT } \\ \text { blue } & \text { bright } \\ \text { 'bright blue' } & \end{array}$

In (9) and (10), STRONG and BRIGHT indicate that the colour is intense. This type of modification of the colour term demonstrates colour saturation in KS. 
KS can express that a colour has a lighter or darker shade, that is, a colour spectrum. This kind of modification is done through the use of WEAK, LIGHT, STRONG and BLACK.

11) BROWN WEAK

brown weak

'light brown'

12) BLACK PURPLE

black purple

'dark purple'

KS can also convey that the colour shade being described is atypical of that colour. This modification is also known as hedging. A KS example is below.
13) BLACK NO
CLOSE
black no
close
'dark blue'

A colour sign can be modified in more than one way. This was seen in:

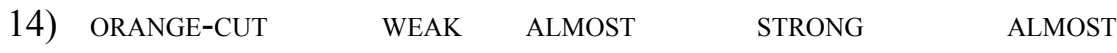 orange light almost bright almost
'light, somewhat bright orange'

The colour being described in (14) can be labelled cobalt yellow in English. The words WEAK, CLOSE and ALMOST STRONG exemplify that KS has colour constructions which show colour saturation, a colour spectrum as well as hedging.

When occurring with nouns, the colour constructions have flexible word order.

$\begin{array}{ll}\text { BIRD } & \text { mouthing: } 1 \\ \text { bird } & \text { yellow }\end{array}$

'yellow bird'

Here, the colour YELLOW occurs after the noun BIRD.

16) BLACK HAIR

black hair

'black hair'

The colour BLACK is before the noun HAIR. 
This is unlike what is seen in examples (9) through (14) where the colour term always occurred first, followed by the modification. If a modified colour term is in a phrase containing a noun, the entire modified colour term can occur either before or after the noun just as a colour term on its own can occur either before or after a noun. This is exemplified in (17) below where the modification WEAK follows the colour term BROWN.

17) CLOTHES BROWN WEAK

clothes brown weak

'light brown clothes'

\subsection{Summary}

KS signers employ several strategies to express colour. There are even various ways of expressing the same colour. Table 2 below shows how KS expresses several colours.

Table 2. Colour terms in Konchri Sain

\begin{tabular}{ll}
\hline & COLOUR TERMS IN KONCHRI SAIN \\
\hline Konchri Sain & English Translation \\
COLOUR & 'colour' \\
COLOUR\#COLOUR & 'colours' \\
BLACK-1 & 'black, dark complexion' \\
BLACK-2 & 'black, dark complexion' (infrequent) \\
WHITE & 'white, fair complexion' \\
COLOUR\#'red' & 'red' \\
(COLOUR) KILL & 'red' \\
(COLOUR) BLOOD & 'red' \\
COLOUR\#'yellow' & 'yellow, orange' \\
(COLOUR) SUN & 'yellow' \\
COLOUR\#'blue' & 'blue' \\
(COLOUR) SKY & 'blue' \\
(COLOUR) TREE & 'green'
\end{tabular}




$\begin{array}{ll}\text { (COLOUR) ORANGE-CUT } & \text { 'orange' } \\ \text { (COLOUR) GRAPE } & \text { 'purple' } \\ \text { DARK_SKIN } & \text { 'brown' } \\ \text { LIGHT_SKIN } & \text { 'pink' }\end{array}$

The forms listed in Table 2 that have the same meaning in English appear to be in free variation. There is no indication that this variation results from phonological, morphological or syntactic triggers. As in numerous other sign languages, many of these colour signs are iconically motivated. On par with de Vos (2011), KS seems to have the fewest colour terms of the four languages used in Jamaica. It should be noted, however, that at least some of the variation found in its colour term system may be related to the language's critically-endangered status rather than to a lack of conceptual categories. In support of this analysis, the sign-bilingual informants often produced the JSL forms in addition to the KS signs to refer to colours.

\section{Conclusion}

This article has examined colour terms in two indigenous sign languages that are typologically unrelated and used in quite different socio-linguistic settings. The KS community is characterised by a high degree of sign bilingualism with Jamaican Sign Language and susbsequent endangerment, while YSL is mainly used as an alternate sign language of an Aboriginal community, and deaf, native signers are few and far between. Notwithstanding these differences, there are also a number of similarities in the social contexts in which these indigenous sign languages are used. While the KS community is now scattered across several villages, they still congregate weekly for church and at other times for other informal gatherings and maintain a dense social network as such. Similarly, compared to urban signing communities, the Yolngu communities that contributed data to this study are relatively small.

In the YSL colour terminology, there are two signs referring to skin colours, MOL 'black/light' and WATHARR 'white/light'. These should not be considered BCT as their semantic scope is limited to the domain of complexion. In addition to these two terms, YSL has at least four lexical colour signs: MOL-1 'black', WATHARR-1 'white', MINY'TJ^MINY'TJI 'red' and BUTHALAK 'yellow'. These four signs are based on the colours used in ceremonies which play an important role in Yolngu culture. YSL 
also allows several modification strategies using non-manual markers such as mouthings and facial expressions to express light and dark shades. When there is no lexical colour sign available, YSL users point to objects in the environment, a common strategy across rural sign languages. This study has also identified the use of kinship terms to refer to colours associated with moieties. This latter referential strategy had not previously been described for any other sign language and might be directly linked to the salience of kinship within Yolngu culture.

The KS system appears to have the most extensive set of lexical colour terms ever reported for a village sign language and marks out five concepts: 'black', 'white', 'red', 'yellow', and 'blue'. It should be noted, however, that there are various alternative strategies for expressing these colour categories (see Table 3). As these alternative forms appear to be used with equal frequency by the participants, it is unclear whether any are psychologically more salient and may thus be considered the BCT for that colour. Two of the KS informants are sign-bilingual between KS and JSL and used the JSL colour terms in addition to the KS signs. It is at present unclear to what extent this factor may have influenced these findings. It was nevertheless ecologically valid to include these data, as most KS signers are nowadays fluent in both sign languages. Similar to YSL, KS has two signs referring to skin complexion (BLACK and WHITE), which express colours of nonhuman entities as well.

A number of differences between KS and YSL can be noted. Like other Aboriginal languages, the degree of saturation might be implied in the lexical colour terms in YSL, but in KS saturation is marked explicitly by the modifiers BRIGHT and DARK. Additionally, YSL signers may mark saturation by the use of facial expression - squinting for low saturation and pouting for high saturation - but this strategy was not attested in the KS data set. Both languages use pointing as a colour description strategy, and these occurrences may be preceded or followed by the lexical sign MINY'TJI in YSL. In KS, colour pointing is usually preceded by the sign COLOUR, or alternatively, by ostensive searching behaviour on behalf of the signer. It thus appears that while village sign languages may adopt pointing as a colour description strategy, it may be conventionalised differently in these communities. Finally, it appears that the KS community allows for more lexical variation in the domain of colour, compared to the YSL community. Without quantification and direct comparison between the data sets, however, it is difficult to substantiate this claim at this stage, or to compare it to the variation found within urban sign languages. One possible difference is that, while the variation in urban sign languages may be socio-linguistically or geographically 
motivated (e.g. linked to specific deaf schools), the variation found in KS is not. In support of this latter analysis, each signer used all of the variant forms to refer to the colours, suggesting that idiosyncrasies play little or no role (cf. de Vos 2011).

All in all, while varying in detail, the YSL and KS colour term systems exhibit some parallels. As with other village sign languages, the sets of lexical items in these two languages appear to be more restricted than those of the surrounding signed and spoken languages, and both communities adopt a strategy of colour pointing. We would, however, like to add a cautionary note, that these proposed differences between urban and rural sign languages may not only lie in the qualitative but also quantitative nature of the data. That is to say, our perspective on rural sign languages may be skewed as there is still marginal data available. Once there are more substantial samples of both urban and rural sign languages accessible, we will be able to state with more confidence what is universal across signed and spoken languages, what is modality-specific, and what might be characteristic of rural sign languages in the domain of colour terminology. This study suggests that much is to be gained by examining individual village sign languages with respect to specific typological domains, as they may exhibit features that are distinct from both urban sign languages and other village sign languages.

\section{Notes}

1. The research project was supported by a DFG research grant to Prof Dr Dany Adone. It is part of the EuroBABEL project on endangered village sign languages, headed by Prof Dr Ulrike Zeshan. We would like to thank all the participants in this study in Galiwin'ku, Mapuru (NT, Australia) and in the St Elizabeth community (Jamaica) for sharing aspects of their culture and language with us. We would also like to thank Dr A. Lowell, our collaborator at the Charles Darwin University, NT, Australia, and Timo Klein, for technical assistance. Many thanks are due to Prof Ulrike Zeshan, Connie de Vos and the anonymous reviewers for their insightful comments.

2. Indigenous Australians who live in the North East Arnhem Land have become famous in the anthropological literature first under the name of Murngin (Warner, 1978 [1937]), and later under the term Wulamba, which has been attributed to Berndt (1955) (Devlin, 1986). Schebeck is said to have introduced the term Yolnu in his original paper in 1968, although this term seem to have had its forerunners in the literature. Chaseling has referred to the people of Arnhem Land with a similar term, Yulengor, in his 1934 book (Wilkinson, 1991, p. 1). Since the 60s, however, the term Yolnu or Yolngu has 
been favoured in the literature and adopted by linguists, anthropologists, and the Indigenous people themselves.

3. Based on a map from van der Wal (1992), this map has been modified to include the position of the homeland centre Mapuru. The dashed line shows the approximate boundary of the Yolngu. (Source: http://livingknowledge.anu. edu.au).

4. E.R. became deaf postlingually most probably due to otitis media (middle ear infection) experienced during childhood.

5. Similarly, Adamorobe Sign Language has a manual sign that means 'sense', rather than 'colour' (Nyst 2007).

6. We would like to thank Inge Zwitserlood for providing the handshape fonts.

7. These clouds are those which typically appear in the sky during the wet season. They signal that a thunderstorm is approaching and consequently excursions should be finished.

\section{References}

Adone, D.

1999 How culture shapes language: The case-study of Yolngu Sign Language. Unpublished manuscript for Anthropological Linguistics. Department of General Linguistics: University of Düsseldorf.

Adone, D.

2002 The use of non-manuals in an alternate sign language. Unpublished manuscript. Department of General Linguistics: University of Düsseldorf.

Berlin, B. \& P. Kay

1969 Basic Colour Terms. Berkeley: University of California Press.

Bimler, D., J. Kirkland \& K. Jameson

2004 Quantifying variations in personal colour spaces: Are there sex differences in colour vision? Colour Research \& Application 29 (2). 128-134.

Bowerman, M. \& S. Levinson (eds.).

2001 Language acquisition and conceptual development. Language, Culture and Cognition: 3. Cambridge: Cambridge University Press.

Christie, M. \& J. Greatorex

2004 Yolngu Life in the Northern Territory of Australia: The Significance of Community and Social Capital. The Asia Pacific Journal of Public Administration. Vol. 26 (1). 55-69 
Cooke, M. \& D. Adone

1994 Yolngu Signing - gestures or language? Darwin, Australia: Batchelor College.

Corbett, G. \& G. Morgan

1988 Colour terms in Russian: reflections of typological cons-traints in a single language. In: Journal of Linguistics 2A. 1-64.

Crasborn, O., E. van der Kooij, D. Waters, B. Woll . \& J. Mesch

2008 Frequency distribution and spreading behavior of different types of mouth actions in three sign languages. Sign Language \& Linguistics 11(1.) 45-67.

Davies, I., G. Corbett, G. Laws, H. McGurk, A. Moss \& M. Smith

1991 Linguistic basicness and dour information processing. International Journal of Psychology 26. 311-327.

Davies, I. \& A. Franklin

2002 "Categorical similarity may affect colour pop-out in infants after all". In: British Journal of Developmental Psychology, 20. 185203.

De Vos, C.

2011 Kata Kolok colour terms and the emergence of lexical signs in rural signing communities. Senses \& Society. Volume 6, Issue 1. $68-76$.

Devlin, B. C.

1986 Language maintenance in a Northeast Arnhem Land settlement. Columbia University Teachers College: PhD Thesis.

Elman, J., E. Bates, M. Johnson, A. Karmiloff-Smith, D. Parisi \& K. Plunkett

1996 Rethinking Innateness: A Connectionist Perspective on Development. Cambridge, MA: MIT Press.

Gentner, D., \& S. Goldin-Meadow

2003 Language in mind: Advances in the study of language and thought. MIT Press.

Gerhardstein, P., P. Renner \& C. Rovee-Collier

1999 The roles of perceptual and categorical similarity in colour popout in infants. British Journal of Developmental Psychology 17. 403-420.

Green, J., G. Woods \& B. Foley

2011 Looking at language: appropriate design for sign resources in remote Australian Indigenous communities. Sustainable data from digital research: Humanities perspectives on digital scholarship. Melbourne, AU: University of Melbourne. 
Haga-Schoonhoven, M., R. Pfau \& B. de Boer

2010 Colour on hands: Phonological markedness of sign language colour terms. Theoretical Issues in Sign Language Research (TISLR 10). West Lafayette, USA.

Hargrave, S.

1982 A report on colour term research in five Aboriginal languages. Darwin: Summer Institue of Linguistics.

Hill, C.

2011 Named and Unnamed Spaces: Colour, Kin, and the Environment in Umpila. Senses \& Society, Vol. 6(1). 57-67.

Johnston, T., \& A. Schembri

2007 Australian Sign Language. Cambridge: Cambridge University Press.

Kay, P. \& L. Maffi

1999 Colour appearance and the emergence and evolution of basic colour lexicons. American Anthropologist 10(4). 743-760.

Kay, P. \& C. K. McDaniel

1978 The linguistic significance of the meanings of basic colour terms. Language 54(3). 610-646.

Kay, P. \& T. Regier

2003 Resolving the question of colour naming universals. Proceedings of the National Academy of Sciences 100. 9085-9089.

Kendon, A.

1984 Knowledge of sign language in an Australian Aboriginal Communitiy. Journal of Anthropological Research. Vol. 40, No.4, Winter, . 556-576.

Kendon, A.

1988 Sign Languages of Aboriginal Australia: cultural, semiotic, and communicative perspective. Cambridge University Press.

Locke, J.

1690/1987 An Essay concerning human understanding. Clarendon Press.

Lucy, J. A.

1997 Linguistic Relativity. Annual Review of Anthropology Vol.26. 291-312.

Maypilama, E. L \& D. Adone

2012 The sociolinguistics of Yolngu Sign Language. Unpublished manuscript. Charles Darwin University. 
Meadows, J.

1974 Disturbed perception of colours associated with localneighbourd cerebral lesions Brain 97, 615-632.

Mollon, J., J. Pokorny \& K. Knoblauch (eds.)

2003 Normal and defective colour vision. Oxford University Press, Oxford, 39-50.

Morphy, H.

1989 From dull to briliant: The aesthetics of spiritual power among the Yolngu. Man, New Series, Vol. 24(1). 21-40.

Nonaka, A.

2004 The forgotten endangered languages: Lessons on the importance of remembering from Thailand's Ban Khor Sign Language. Language in Society, Vol. 33(5). 737-767.

Nyst, V.

2007 A Descriptive analysis of Adamorobe Sign Language (Ghana). Utrecht, The Netherlands: LOT.

O'Reilly, S.

2006 Indigenous sign language and culture: the interpreting and access needs of deaf people who are Aboriginal and/or Torres Strait Islander in far north Queensland. Australia: Sponsored by ASLIA, the Australian Sign Language Interpreters Association.

Palmer, S. E.

1999 Colour, consciousness, and the isomorphism constraint. Behavioral and Brain Sciences 22.923-989.

Pfau, R.

2012 Manual communication systems: evolution and variation. In R. Pfau, M. Steinbach, \& B. Woll (eds.), Sign language. An international handbook. Berlin: Mouton de Gruyter.

Pinker, S., \& Bloom, P.

$1990 \quad$ Natural language and natural selection. Behavioral and Braiin Sciences 13. 707-784.

Posner, M. I. \& M. E. Raichle

1994 Images of Mind. New York: W.H. Freeman.

Roberson, D., I. Davies. \& J. Davidoff

2000 Colour categories are not universal: Replications \& new evidence from a Stone-age culture. Journal of Experimental Psychology: General 129. 369-398.

Rosch-Heider, E. \& D. Olivier

1972 The structure of the colour space in naming and memory for two languages. Cognitive Psychology 3. 337-354. 
Rumelhart, D. \& J. McClelland

1986 Parallel distributed processing: Exploration in the microstructure of cognition. Vol.1: Foundations. Cambridge, MA: MIT Press.

Saunders, B. \& J. van Brakel

1997 Are there nontrivial constraints on colour categorization?. Behavioral and Brain Sciences 20. 167-228.

Shepard, R.

1994 Perceptual-cognitive universals as reflections of the world. Psychonomic Bulletin \& Review 1. 2-28.

Steels, L. \& T. Belpaeme

2005 Coordinating Perceptually Grounded Categories through Language. A Case Study for Colour. Behavioral and Brain Sciences 28 (4). 469-489.

Stokoe, W.

1978 Sign Language Structure. Rev.ed. Silver spring, Md.:Linstok.

Stokoe, W.

1987 Lexical indicators of cultural change. In: Kyle, J. (ed.) Sign \& School Avon, England: Multilingual Matters, Ltd. 25-29.

Thompson, E.

1995 Colour Vision. New York: Routledge. van der Wal, A.

1992 Structure and Fuction in Gupapuypu, a Yolyu dialect of Northeast Arnhemland. Newcastle, N.S.W.: Ph.D. Thesis, University of Newcastle.

Warner, W.

1978 Murngin Sign Language. In D. Umiker-Sebeok, \& T. Sebeok (Eds.), Aboriginal Sign Languages of the Americas and Australia. Vol. 2, 389-392. New York: Plenum Press.

Watson, H. \& Chambers, D.

1989 Singing the land, signing the land. Geelong, Victoria, Australia: Deakin University Press.

Wierzbicka, A.

2006 There are no 'colour universals', but there are universals of visual semantics. Antrhoplogical Linguisitcs 47 (2). 217-244.

Wilkinson, M.

1991 Djambarrpuyzu. A Yolyu Variety of Northern Australia. Sydney: University of Sydney. 
Woodward, J.

1989 Basic colour term lexicalization across sign languages". Sign Language Studies 63. 145-152.

Zeshan, U.

2011 Sign Language Typology - New Evidence from Sign Languages in Village Communities. Presented at the IVth International Conference of the French Cognitive Linguisitics Association: Lyon.

Zorc, D.

1986 Yolyu-Matha Dictionary. Darwin, N.T. Australia: School of Australian Linguistics, Darwin Institute of Technology, Batchelor, N.T. 


\title{
Demarcating generations of signers in the dynamic sociolinguistic landscape of a shared sign-language: The case of the Al-Sayyid Bedouin
}

\author{
Shifra Kisch
}

Signing communities emerging in the context of high incidences of deafness have attracted the attention of scholars of different disciplines, most notably in Deaf studies and sign language linguistics. Linguists believe that sign-languages emerging in these circumstances may provide a rare opportunity to study new language isolates, the study of which holds the promise of contributing to some of the key questions of modern linguistics, particularly with regard to the conditions that engender language (Senghas 2005; Arbib 2009; Meir et al 2010a).

However, the study of emerging sign languages has been criticised for exclusive emphasis on the innate language capabilities of the human brain and disregard for social, pragmatic, and environmental influences (Russo and Volterra 2005; Fusellier-Souza 2006; Arbib 2009; Nyst in press). Two studies in particular have become renowned for their claims of witnessing the emergence of (signed) language in the absence of existing language models. The study by Kegl et al (1999) documented the emergence of Nicaraguan Sign Language (NSL) by deaf children brought together in a school for deaf students in Managua. Senghas et al (2004:1779) argued that NSL has arisen within a community that lacked exposure to a developed language. Meir and Sandler (2008:292), studying the Al-Sayyid Bedouin Sign Language (ABSL), have described it as a "Language out of nothing...developing without a language model". ABSL is one of the sign languages emerging in the context of high incidences of deafness.

Signing communities and sign languages emerging in the context of exceptionally high rates of deafness have been referred to using a variety of terms (for discussion of these alternative terms see Kisch 2008) ${ }^{1}$. In my study of the Al-Sayyid Bedouin, I have introduced the term shared signing community (Kisch 2000, 2008), later adopted by Nyst (in press) to correspondingly denote shared sign languages.

In shared signing communities deaf and hearing infants are exposed to signing from birth, within the family environment, with additional (deaf 


\section{Shifra Kisch}

and hearing) adult models in the community. In these unique sociolinguistic conditions, signing as a practice does not single out deaf people. Thus, the term is meant to capture and emphasise one of the prominent features that these cases have in common: signing is not what deaf people "do", but rather a medium for deaf and hearing people to communicate.

Consequently, a large proportion of language users (namely all hearing signers), are bilingual in the local sign language and the local spoken language. In this respect, no shared sign languages can be considered to develop without exposure to a language model (for similar emphasis see Arbib 2009).

Demarcating generations of signers is an important element in the study of young sign languages ${ }^{2}$. The number of times such an emerging linguistic system is passed down to a new generation is considered significant for the identification of new linguistic properties (Senghas 2005; Goldin-Meadow 2005) and practices. However, intergenerational transmission is just one of the sociolinguistic features involved in shaping a language. Although linguists hope to identify independent developments within a language, it seems imperative to consider communicative input broadly as well as the possibility of contact-induced change. Both have too often been overlooked.

The challenges involved in identifying generations of signers are different when considering a language emerging within a student community as compared with a shared signing community. NSL was passed to a new successive cohort of 15-20 learners each year (Senghas et al 2004; Senghas 2005). Though controversies arise with regard to the communicative input that students experienced before entering the school and the possible influences of gestures and written language (Polich 2005; Russo \& Volterra 2005), their exposure and contribution to the emerging signing community of NSL was clearly marked by their year of enrollment ${ }^{3}$. By contrast, signers of shared sign language and ABSL are exposed to the emerging language early in life, in a multigenerational family environment. Sandler and her colleagues (Sandler et al 2005, 2011; Aronoff et al 2005, 2008; Padden et al 2010; Meir et al 2010a; Meir 2010) base their analysis of ABSL on their identification of three generations of signers. They define these generations in very general and often inconsistent terms ${ }^{4}$, and do not present their considerations in choosing these classifications.

This paper examines the challenges involved in identifying generations of signers in shared signing communities. Based on the analysis of the diverse available communicative input and the observed shifting sociolinguistic landscape of the Al-Sayyid shared signing community, four generations of signers are identified. The purpose of this exercise is twofold: to inform the 
further investigation of ABSL and to serve the study of comparable shared sign languages by identifying the social factors that transform the sociolinguistic networks of signers, too often assumed to be embedded simply in primary kin-networks.

The case of Al-Sayyid illustrates the processes by which signers are embedded in multiple language communities. My intention here is not to determine the actual impact such sociolinguistic dynamics might have on the grammatical skills (of different generations) of ABSL signers, but rather to facilitate the identification of such variation and its possible correlates.

The first part of this paper briefly introduces the history of the Al-Sayyid descent-group, and examines the usefulness of kinship diagrams. It addresses the confusion often caused by mistaking structural generations of descent for social or cultural generations, and considers the relevance of these categories and kinship diagrams to the task of identifying sociolinguistic generations.

The second part presents the four generations of deaf Al-Sayyid signers, including four sub-cohorts (summarised in Table 1). Before presenting the detailed profile of these generations, the case of hearing signers is addressed, including my suggested guidelines for incorporating hearing signers into this structure.

The third and last part examines the major social factors that have transformed the sociolinguistic landscape of Al-Sayyid deaf signers. Schooling introduced deaf students to a new sign language and signing community and reduced the social space shared by deaf and hearing signers in the Al-Sayyid shared signing community. Schooling and the subsequent marriage and labour patterns transformed signers' sociolinguistic networks.

This analysis is based on data collected over a decade and a half of anthropological fieldwork and participant observation among the Negev Bedouin and a total of over 30 months of in-residence fieldwork based in Al-Sayyid. Several demographic and genealogical surveys focusing on Al-Sayyid's deaf population were conducted in 1995-1999, 2004-5, 2007 and most recently in 2011, and were supplemented by data obtain from the ministry of education and ministry of interior.

When I first visited the Al-Sayyid in 1995 I could communicate at a basic level and did not need to employ an interpreter. However, given the collaborative nature of the ethnographic method and the common practices of sign/ speech mediation among the Al-Sayyid (Kisch 2000;2008), I could rely on ad hoc spontaneous interpreting when necessary. In the course of my first fieldwork months I could increasingly communicate directly in ABSL, my main mentors being the members of my extended host family, at the time including 2 deaf children, 2 young deaf adults, an elderly deaf grandmother, 
and many competent hearing singers. In my immediate host family, only one member could speak Hebrew, serving as a compelling incentive for improving my competence in the locally spoken Arabic dialect ${ }^{5}$. To improve my communication with other (mostly non-Al-Sayyid) deaf signers encountered throughout my fieldwork, I also took three ISL courses, two of which were provided by qualified deaf instructors at The Institute for the Advancement of Deaf Persons in Israel (DPII).

This paper is based on data collected by means of fieldwork interaction and observation conducted predominantly in the locally spoken Arabic dialect and in ABSL, occasionally in ISL, and rarely in Hebrew. The latter two are used by interlocutors in specific contexts such as encounters involving non-Arabic-speaking service providers or young deaf adults' who use ISL with schoolmates, and Hebrew for Short Message Service. Unless otherwise stated, the conclusions below are based on participant observations of recurrent and shifting linguistic behavior over an extended period of time. When analysis is based on self-reported behaviors, semi-structured interviews, or singular events, this is explicitly stated.

\section{The Al-Sayyid Descent-Group}

The Bedouin are former nomads and the native Arab inhabitants of the Negev. Al-Sayyid is the name of a large descent group that inhabits one of the many Bedouin settlements in the Negev. Until recently it was formally unrecognised by the state and therefore still lacks basic infrastructure and facilities. The number of inhabitants is estimated to be over 4500, with nearly 130 deaf individuals distributed throughout the Al-Sayyid lineages. For the purpose of this article an updated survey listing all deaf individuals was conducted in the summer of 2011.

All Al-Sayyid are kin related and are named after their common ancestor Al-Sayyid, who migrated to the Negev nearly 200 years ago. Al-Sayyid had sons and daughters from two wives. Five of these sons (level 2, fig. 1) are today considered the apical ancestors of the Al-Sayyid's major lineages. At the time, finding spouses for the founders children was a crucial and difficult affair, as was the case for other migrants who were not considered of noble (Bedouin) descent. It was therefore a great relief when his grandchildren (the 3rd generation, level 3 in fig. 1), could intermarry, following the practice of cousin marriages. (To this day consanguineous marriages are the prevailing marriage pattern among the Negev Bedouin). Four of these initial cousinmarriage unions bore the first deaf descendants among the Al-Sayyid; these constitute Al-Sayyid's 4th generation ${ }^{6}$, represented in level 4 of fig. 1. 
Hadra and her three deaf brothers were the first deaf descendants of the Al-Sayyid family. They were born between 1924-1940, under the British mandate of Palestine, preceding the establishment of the state of Israel, and grew up under significantly different circumstances than their, by now, over four and a half thousand relatives and descendants.

Hadra, 1924-2003 (fieldnote excerpts)

When I first met Hadra in the winter of 1995 she was in her early seventies, her husband had passed away and she was living with her youngest son, his wife and their ten children. Nearby were the homes of her elder sons, one of her daughters and several married grandchildren, all surrounding a small dale with a plot of scarcely arable land.

"I no longer leave this dale, not even to go to the doctor. They can all come to me now, and so did you" she laughed.

"If you sit here with us you will learn" she responded to my wish to learn the local sign language, "but I don't sign like that!" she warned me sharply, she then used a restricted signing space under her chin to mimic the small and quick fingerspelling she had seen her grandchildren use. The deaf and hearing children who were sitting with us around the fire, sheltered from the rain in the half open shed, all burst out laughing.

Only one of Hadra's (hearing) sons attended school, and his sign name was appropriately "[the one who can] write". Hadra's grandchildren all went to school. Her hearing grandchildren attended the school on the top of a small hill in the center of the village. The deaf children however were daily bused out of the village to a school for the deaf in the nearby city of Beersheba. Two of Hadra's deaf grandchildren -by then already graduated and married - were among the first Bedouin cohort of students in the early 1980s. Her younger deaf grandchildren were still attending this school.

Sitting around the fire was also one of Hadra's deaf grandchildren, drawing big Hebrew letters in the dry earth; he was figuring out the names of his cousins with the help of one of his hearing cousins who was old enough to have learned some Hebrew (as a second language at school). The two cousins were absorbed in their exchange; the hearing girl (a fluent signer) was intrigued to learn the fingerspelling he learned at school and the deaf boy needed her help; at the school for the deaf they only learned Hebrew. It was their signing that Hadra was referring to and poking fun at just now. She has also observed her deaf grandchildren immersed in long conversations in ISL, and watched simultaneous ISL interpreting on TV, all of which she reported not to understand the smallest bit of. "NOTHING UNDERSTAND."

It might seem most straightforward - even redundant - to classify signers according to generations of descent; Hadra- being the first deaf person 


\section{Shifra Kisch}

among the Al-Sayyid, her children as second generation (hearing signers, in this case), her (deaf and hearing signing) grandchildren, third generation, and so on.

However, when considering a way to account for the different generations of signers in the Al-Sayyid signing community as a whole, generations of descent will not suffice. Actually, such a classification would run into confusion even within Hadra's own descendants, given the intra-generational age gap that is characteristic of societies with high fertility rates. Additionally, due to prevailing endogamous marriage patterns, individuals are often related in more than one direction and degree, and a single individual can simultaneously be traced to different generations of descent. In a polygynous society this is further complicated by the age/ generational gaps between husband and wives and of half-siblings.

The relatively young age of the Al-Sayyid descent-group allows us to trace practically all individuals back to their common ancestor. But from the third generation onwards the dense kin relations form an increasingly convoluted genealogical network. Some of Hadra's grandchildren overlap in age with her own children while others overlap in age with her great-grandchildren. The birth dates of Hadra's grandchildren born from just one of her sons span 41 years. Thus, for the purpose of considering intergenerational transmission of language, generations of descent might be not only insufficient but misleading.

To avoid such confusion I will first distinguish two common denotations of the term generation, namely, structural generations of descent versus social or cultural generations of actual contemporaries. In kinship studies the concept of a generation generally refers to structural generations. Note that it is erroneous to consider either of these categories to be biological. A structural generation includes those descendants with the same distance from their apical ancestor. In conventional kinship diagrams (see example fig. 1) such generations are represented horizontally and are staked vertically according to descent. Social or cultural generations, as a broader social category, are based on groups of contemporaries. These are assumed to have shared socio-cultural experiences or at least to have been subject to similar social settings and developments. Years of birth can serve as a starting point to group contemporaries but it is essential to identify their shared circumstances. 
While both categories are relevant for the task of identifying generations of signers, generations of signers are in essence social generations that may or may not correspond to structural generations of descent. Confusion may arise with regards to shared signing communities where kinship is indeed a dominant factor in shaping social relations. However, even when kinship is observed to be a primary mode of social organization (and frame of meaning), this is often context-dependent and seldom the only source of networks of affiliation and collaboration. For instance, when kin-endogamy is preferred and serves as a cultural idiom, the actual rates, as well as the flexible nature of the practice, are often downplayed in local (emic) discourse. In practice, kin-endogamy is rarely sweeping; even high consanguinity rates such as those found among Negev-Bedouin (with over $60 \%$ of marriages between relatives $^{7}$ ) leave many marriages involving non-kin. An additional source of confusion is related to the common (etic) representation of kinship; kinship diagrams seemingly provide an overview of such relations but may also be a source of confusion.

Kinship diagrams- like most representations- can only illustrate a limited number of features simultaneously. In fact, many conventional kinship diagrams are designed to illuminate prototype social structures rather than actual social relations. Below two diagrams are presented, a conventional (fig. 1) and a modified skewed ${ }^{8}$ kinship diagram (fig. 2), both depicting the same selected Al-Sayyid relatives: apical ancestors and first deaf descendants. While the diagrams convey important information regarding the Al-Sayyid genealogy, they were also designed to demonstrate how ambiguous conventional diagrams can be, that is if one hopes to extract information from them regarding generations of signers, or actual social relations. Generations of coevals cannot be inferred from the level of descent in such a conventional chart, for instance those on level 5 of fig.1(for whom Al-Sayyid the founder is the grandfather of their paternal- grandfather) range in age from 1950 2011. This is made visible in the skewed chart (fig.2). While this diagram is designed to facilitate identification of generations of signers based on their contemporaries, such skewed diagrams are not suitable to conclude who people actually interacted with. For instance, patrilocal residence is partially represented by locating offspring under their father rather than their mother, but this is not sufficient to determine actual proximity. 


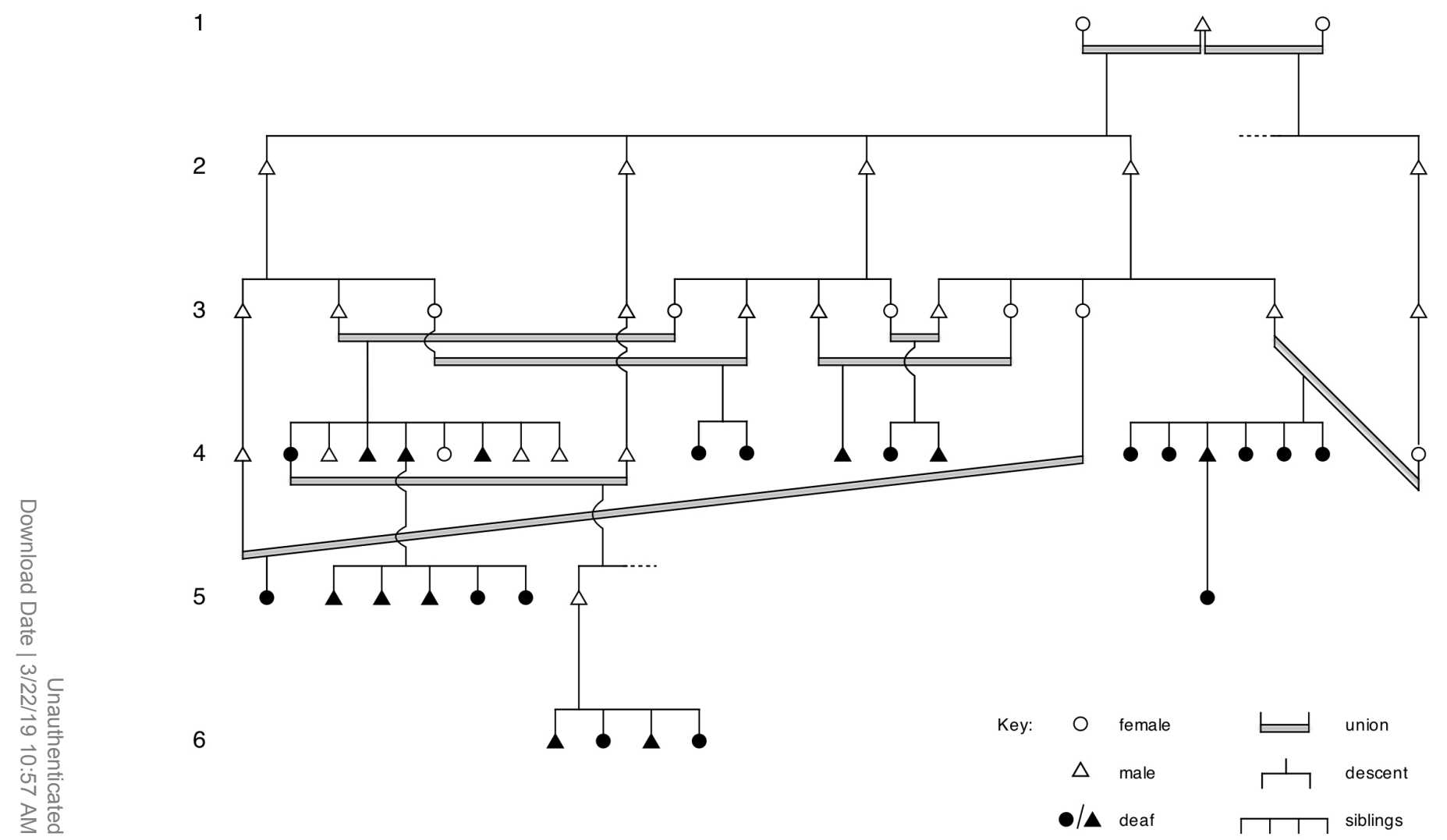

Figure 1. Horizontal ("conventional") kinship diagram of Al-Sayyid apical ancestors and first deaf descendants, representing selected individual of 6 out of 8 structural (patri)generations of descent. 
Demarcating generations of signers: The case of the Al-Sayyid Bedouin 95

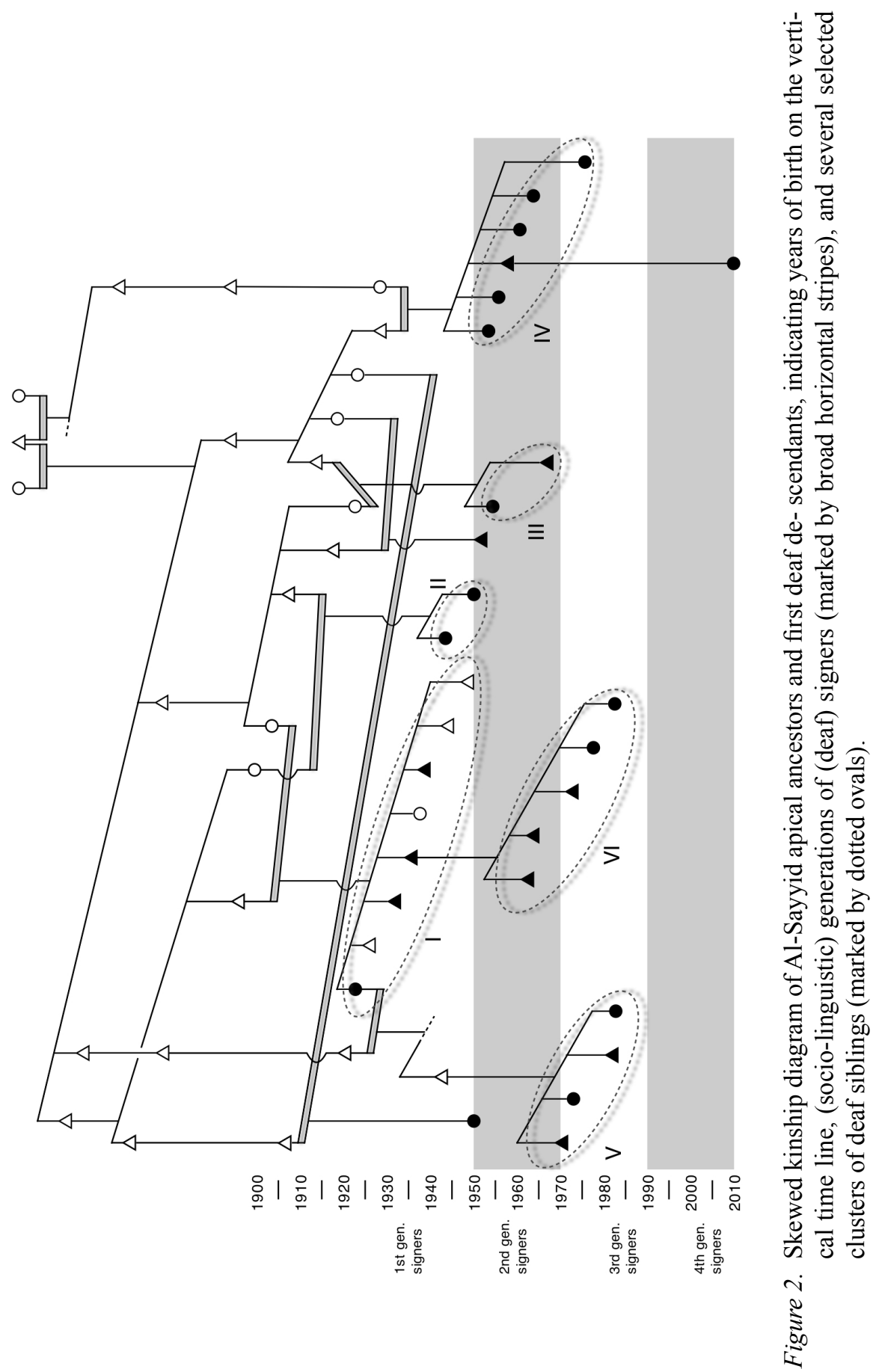


Annotations $^{9}$ for Figures 1 and 2:

1. Both diagrams adhere to the local rule of patrilineal descent. Distribution to generational levels in Figure 1 will alter if computed through matrilineal or bilateral descent.

2 Only a selection of deaf relatives and their hearing ancestors are represented; consequently, the diagram does not represent the actual ratio between deaf and hearing relatives.

3. Figure 1: Level 1 represents the apical ancestor of the Al-Sayyid descent group; in level 2 only sons that are considered to be the apical ancestors of the Al-Sayyid lineages are represented. All deaf individuals of the fourth (patri) generation are represented. Only a few deaf individuals of subsequent generations are represented, and descendants of the 7th and the 8th (patri) generation are not represented at all.

4. The skewing of Figure 2 is based on birth years, which were registered for all individuals born after 1950 and for a few older individuals. Other years of birth are estimated, and for those born before 1880, are unknown.

5. Figure 2: All deaf signers of the first and second generation are represented in this diagram, but only very few of the hearing ones. Only a random selection of third generation deaf signers is represented. For instance, the deaf siblings in cluster $\mathrm{V}$ are only 4 out of a total of 32 offspring of their father, himself one of the 19 offspring of his father. Representing them all would result in an unmanageably large diagram.

\section{Generations Of Signers Among The Al-Sayyid}

Age determines one's contemporaries, both peers and existing adult models, and in the case of Al-Sayyid also neatly corresponds to school attend- ance. Age and schooling therefore form the basis for distinguishing the four generations and sub-cohorts of deaf Al-Sayyid signers (summarised in table 1 below). Schooling is prominent due to its role in transforming the sociolinguistic landscape of entire age groups. Moreover, only a small minority of the deaf Al-Sayyid have not experienced some form of schooling (14 out of the 134 deaf descendants among all generations of Al-Sayyid). Although schooling for deaf students has been re-structured several times within a relatively short period, these changes involved entire cohorts of students, and very few deaf students (10 so far) have had a significantly different trajectory than their age group. The age range of approximately 20 years for each generation 
is also in accordance with the average generational length among the Negev Bedouin today. Before proceeding to the profiles of the distinct generations of signers summarised in the table below, the generational classification of hearing signers -not included in this table - should be considered.

\subsection{Hearing signers}

The introduction of deaf education in the early 1980's was a major factor in transforming the Al-Sayyid sociolinguistic landscape. Not only did it introduce deaf students to a new sign language and a new sign community - it restricted the daily interaction between deaf and hearing peers and consequently reduced the social space shared by deaf and hearing signers in the Al-Sayyid shared signing community.

However, this should not be understood to reduce the importance of hearing signers; on the contrary, hearing signers should be considered important participants in the study of shared sign languages, and it is equally important to recognise which generation of signers they belong to. While fewer hearing Al-Sayyid may be regularly exposed to signing, those that do sign are increasingly vital for the maintenance of ABSL, as is typically the case for other shared sign languages ${ }^{10}$. From its onset, hearing signers participated in the development and intergenerational transmission of the local signing system. It has mistakenly been assumed that deaf Al-Sayyid children are typically "raised in homes with at least one older deaf person who signs" (Senghas 2005:R464). In fact fewer than half (22/49) of the deaf signers of the second and third generations grew up in homes with older deaf signers: nine had deaf parents, and the rest had one or more older deaf siblings (some only 3 years older). More than half (27/49) of the second and third deaf generation signers grew up in homes with no older deaf person, but rather acquired much of their initial signing from hearing signers.

Roughly a third of the hearing population is observed to sign regularly. From early on hearing signers outnumbered deaf signers. Even the most conservative estimate, counting only the most immediate hearing signing relatives of deaf individuals, would amount to several hundred, compared with 130 deaf signers. A minimum estimate of 700 signing relatives is based on a survey I conducted that considered only those hearing signers who were regarded as competent signers by other household members (including at least one deaf relative). This survey considered only immediate relatives such as siblings, half-siblings, partners, and children of deaf individuals. Furthermore, ethnographic data indicates a significant number of proficient hearing signers without immediate deaf relatives. 


\section{Shifra Kisch}

All hearing signers are bilingual, having acquired their signing skills along with the development of their spoken language skills in Arabic, or as a second language. The latter is the case for those whose sign language skills were enhanced only later in life, such as some of the elder siblings or partners of deaf individuals. While many linguists may be primarily interested in samples of signed output from fluent signers, the more general practices of signing should not be overlooked. It is not uncommon for hearing signers to sign and speak simultaneously, in various combinations including signing their speech. Such practices can also be observed for fluent signers ${ }^{11}$, depending on their interlocutors. Less fluent hearing signers will regularly sign and speak at the same time.

It is also important to keep in mind that the actual ease of communication between deaf and hearing, as well as the status of signed communication, does not merely depend on the number of fluent hearing signers (Kisch 2000, 2008). Many signers not considered fluent can still communicate practical matters with relative ease. In addition, the hearing members of the community who are considered poor or non-signers recognise sign language as a proper language and often demonstrate awareness of the pragmatics of signed communication. Moreover, they can easily find hearing signers to act as mediators, translators, or for instruction in improving their own signing skills (for more on such intermediary practices see Kisch 2008)

Generally hearing signers can be grouped with their corresponding age group of deaf signers. Yet, for both hearing and deaf signers, age cannot be considered alone; generations are grouped taking into account their shared social networks and sociolinguistic settings.

Until recently the schooling of hearing signers did not play a role in (differential) exposure or use of sign language. Schooling may become increasingly relevant for hearing signers of the youngest generation (4.2) now that many have deaf schoolmates. However, for most of the hearing Al-Sayyid particular social relations, rather than cohorts of students, are the primary factor in determining their exposure to signed communication and eventual signing skills. Thus, it is important to examine their social networks and establish which signing relatives and individuals they communicated with regularly as indicated by the recording of genealogies, residential patterns, observations and interviews.

Both deaf and hearing signers refer to ABSL with a variety of terms: "khurs" (literally 'mute [language]'). LOCAL/OUR-SIGNS or ARABSIGNS, are used both in Arabic and ABSL, (recently some deaf signers have also started using an ISL sign for BEDOUIN-SIGNS). JEWISHSIGNS or SCHOOL-SIGNS (in ABSL) and "Jewish (sign) language" and 
"School (sign) language" (in spoken Arabic) are used to refer to ISL. ISL is generally perceived by both deaf and hearing people to be the language of education and it is increasingly understood to be prestigious. Some deaf and hearing Al-Sayyid consider SCHOOL-SIGNS to be of a higher status, comparable to the perceived diglossic hierarchy between 'high' (classic) literary Arabic language and 'low' (colloquial) languages. Although this can be understood to imply the devaluation of ABSL there are no explicit negative attitudes expressed towards ABSL and bilingualism is generally valued. ISL is also increasingly associated with deaf sociality. While both deaf and hearing signers commonly refer to ABSL as "our signs," hearing signers also increasingly refer to ISL not only as 'Jewish' or 'school' signs but as "their signs," referring to deaf people, and thus, to something not shared by deaf and hearing.

Some hearing signers have acquired some ISL vocabulary (such as colour and place names) but none has been observed, or claims to be capable of, conversing in ISL. They recognise ISL as distinct from ABSL and unintelligible, and unlike many deaf signers, refrain from judging others' ISL proficiency:

Hadil is 10 years old, she is deaf and so are her father and several of her many maternal uncles and aunts. Her hearing mother and deaf father communicate in ABSL, as do most of her immediate relatives. With her 30 years old deaf aunts Hadil regularly communicates in ISL. Hadil also makes lucid assessments of the signed proficiency of her deaf and hearing relatives. She was praising the (ABSL) signing of the hearing wife of her deaf uncle: "she signs very well, she is the best. But school signs - nothing!". About her older deaf uncle she says "his school signs are so-so, he uses Arabic sings most of the time anyways. But my [hearing] mother? beware, she can understand a good deal of school signs!".

Table 1. Generations and (sub)cohorts of deaf Al-Sayyid signers. (NIV refers to the Hebrew Deaf School)

\begin{tabular}{|c|c|c|c|c|}
\hline \multicolumn{2}{|c|}{$\begin{array}{l}\text { Generation / } \\
\text { cohort }\end{array}$} & $\begin{array}{l}\text { Years } \\
\text { of } \\
\text { birth }\end{array}$ & $\begin{array}{l}\text { Number } \\
\text { of deaf } \\
\text { signers }\end{array}$ & Major sociolinguistic factors \\
\hline \multicolumn{2}{|l|}{$1+2$} & & 17 & \\
\hline 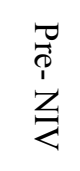 & 1 & $\begin{array}{l}1924- \\
1950\end{array}$ & $\begin{array}{l}\text { (of whom } \\
5 \text { are } \\
\text { deceased) }\end{array}$ & $\begin{array}{l}\text { First deaf descendants. } \\
\text { - No signed input other than the homesigns } \\
\text { developed by them and their hearing relatives. } \\
\text { - Clusters I and II (fig. 2) }\end{array}$ \\
\hline
\end{tabular}




\begin{tabular}{|c|c|c|c|c|}
\hline $\begin{array}{l}\underset{0}{0} \\
i \\
Z \\
Z\end{array}$ & 2 & $\begin{array}{l}1950 \\
1953- \\
1969\end{array}$ & $\begin{array}{r}1 \\
10 \\
11\end{array}$ & $\begin{array}{l}\text { Second generation } \\
\text { - The first likely to have had adult (hearing or } \\
\text { deaf) models. } \\
\text { - Cluster VI (fig. 2) deaf parents and paternal } \\
\text { uncles and aunt. } \\
\text { - } 4 \text { of the siblings in Cluster IV (fig. 2) were the } \\
\text { first to attend formal education (albeit for one } \\
\text { year only) in a West Bank Palestinian school } \\
\text { for the deaf. }\end{array}$ \\
\hline 3 & & $\begin{array}{l}1970- \\
1991\end{array}$ & 38 & \\
\hline \multirow{3}{*}{ Z } & 3.1 & $\begin{array}{l}1970- \\
1980\end{array}$ & 10 & $\begin{array}{l}\text { Early Niv [Hebrew deaf school] } \\
\text { - Signers exposed from young age to input in } \\
\text { ABSL } \\
\text { - All but } 2 \text { spent } 5-10 \text { years at Niv school for the } \\
\text { deaf, exposed to ISL and written Hebrew. } \\
\text { - } 1-2 \text { deaf instructors. } \\
\text { - Signers in this cohort differ greatly in their ISL } \\
\text { fluency. }\end{array}$ \\
\hline & 3.2 & $\begin{array}{l}1981- \\
1991\end{array}$ & 26 & $\begin{array}{l}\text { Late Niv [Hebrew deaf school] } \\
\text { - Signers exposed from a young age to input } \\
\text { in ABSL, and to ISL and written Hebrew, in } \\
\text { school. } \\
\text { - Average of } 13 \text { years of schooling, including } \\
\text { Deaf instructors. } \\
\text { - Additional exposure to ISL on TV. } \\
\text { - Many use Internet, SMSs and other written } \\
\text { communication in Hebrew. } \\
\text { - Most men continued education in residential } \\
\text { school for the deaf; many of the women } \\
\text { participated in vocational training or deaf } \\
\text { empowerment programs in ISL. All programs } \\
\text { included Deaf instructors. } \\
\text { - Often prefer communication in ISL among } \\
\text { themselves. } \\
\text { - Deaf-deaf marriages: } 7 / 14 \text { marriages of deaf } \\
\text { women are to a deaf partner. }\end{array}$ \\
\hline & & & $\begin{array}{l}2 \\
(1 \\
\text { deceased })\end{array}$ & $\begin{array}{l}\text { Deaf individuals born with Cerebral Palsy. } \\
\text { Neither attended school in the village or with } \\
\text { their deaf peers; one has been institutionalised } \\
\text { at a young age, the other deceased in his late } \\
\text { teens. }\end{array}$ \\
\hline
\end{tabular}




\begin{tabular}{|c|c|c|c|c|}
\hline \multicolumn{2}{|l|}{4} & $\begin{array}{l}1992- \\
2011\end{array}$ & 79 & \\
\hline \multirow[b]{2}{*}{$\begin{array}{l}\overline{0} \\
0 \\
\bar{T} \\
Z \\
Z \\
Z\end{array}$} & 4.1 & $\begin{array}{l}1992- \\
1999\end{array}$ & 32 & $\begin{array}{l}\text { Andalus [Arabic schools] } \\
\text { - New classes for deaf students in Arab schools. } \\
\text { - ISL vocabulary used by teachers to codify/sign } \\
\text { spoken and written Arabic. } \\
\text { - One deaf staff member. }\end{array}$ \\
\hline & 4.2 & $\begin{array}{l}2000- \\
2011\end{array}$ & 47 & $\begin{array}{l}\text { Al-Sayyid [local village school(s)] } \\
\text { - Classes for deaf students in Al-Sayyid local } \\
\text { school(s). } \\
\text { - Staffed by several deaf teaching assistants (of } \\
\text { the late-Niv } 3.2 \text { generation) } \\
\text { - Increased interaction with hearing signing } \\
\text { peers in ABSL. } \\
\text { - Practically no direct contact with first } \\
\text { generation deaf signers. } \\
\text { - } 14 \text { children with Cochlear Implants who are } \\
\text { increasingly segregated from their deaf peers. }\end{array}$ \\
\hline \multicolumn{4}{|c|}{ Total deaf signers: } & $134(-6$ deceased $)$ \\
\hline \multicolumn{4}{|c|}{ Total (living) congenitally deaf } & $130\left(=134-6\right.$ deceased $\left.+2^{12}\right)$ \\
\hline
\end{tabular}

\subsection{Deaf signers}

\subsubsection{First generation of Al-Sayyid signers}

The first generation of signers includes the first deaf siblings (cluster I, fig. 2: Hadra and her 3 deaf brothers) and 2 deaf sisters. All but the youngest of them were born during the British mandate of Palestine. Interviews conducted in the late 1990s with five out of these six individuals as well as with some of their hearing relatives confirm that while growing up, they were not exposed to signed input other than the homesigns developed by them and their hearing relatives. Indeed the youngest of these siblings were exposed to a slightly more elaborate signing system used by their elder siblings (when her youngest deaf brother was born Hadra was already 16 years old) ${ }^{13}$.

Two deaf sisters (cluster II, fig. 2) can be considered first generation signers despite being younger than Hadra and her deaf brothers. They migrated with their families to Jordan at a young age, and returned following the arranged marriage of one of the sisters. While one might claim their homesigns developed separately, their parents (both siblings of Hadra's parents) migrated after their deaf niece and nephews were born and had been exposed to their 
emerging linguistic system. During their years in Jordan their communication was largely restricted to homesigns with their parents and a few other relatives. The sisters report having had no formal education or contact with other deaf people before returning to Al-Sayyid.

The majority of the Al-Sayyid remained in the Negev, under the patronage of the Qderat alliance. Most Bedouin fled or were driven out of the Negev during the aftermath of the 1948 war (Marx 1967; Falah 1989). Those remaining were confined to a restricted area and kept under military rule until 1966. However, within the closure zone Bedouin interacted in various ways (Marx 1967). One of Hadra's brothers told me that during this time he befriended a deaf man from the neighbouring Qderat settlement (see the sociolinguistic sketch by Kisch, this volume, for more details).

Thus it is not the case, as claimed elsewhere (Aronoff et al 2005:31), that the first two generations had no contact with other deaf people; this is even less true for the second generation discussed below. However, other than occasional contact with a few neighbouring deaf Bedouin and the partial Arabic literacy of one of the first deaf siblings ${ }^{14}$, there is no reason to believe these first-generation deaf signers were exposed to other (sign) languages, except of course the Arabic-speaking hearing signers with whom they created and shared their homesigns from the onset.

\subsubsection{Second generation signers}

The 11 deaf individuals classified as second generation signers were born between 1950 and 1969: they include several paternal cousins (clusters III and IV in fig. 2), their maternal cousins growing up in different Al-Sayyid lineages, and two of the sons of a first generation deaf signer (cluster VI in fig. 2).

This context clearly provides opportunities for intergenerational contact. However, other than the two sons of the deaf father, this generation did not grow up in households with older deaf or hearing signers. I conducted interviews and consulted my records of genealogies, residential patterns and interpersonal relations to establish how frequently these deaf children or their hearing parents and siblings actually communicated with the available adult models (deaf and hearing).

Let us consider the mother of the deaf siblings in cluster III (fig. 2): her much older half-sister bore the first deaf siblings (cluster I, fig. 2), in fact she herself is the same age as her niece Hadra. She does not recall spending 
much time visiting her much older half-sister or interacting with her deaf nephews, but she is likely to have picked up some signing that inspired her initial signs with her own deaf children. It is thus possible that with limited or no direct contact, new homesigns evolved in several families. These homesigns were inspired by hearing signers that had occasional exposure to the earlier signing of relatives.

The first and second generation signers all grew up before deaf education was offered to Bedouin in the Negev. In fact, their hearing siblings did not always attend school either. The first elementary school was opened in Al-Sayyid in the early 1960s and was attended by mainly boys. A few deaf boys had joined their hearing siblings but soon stopped attending, when they realise the hearing teacher lacked the competence to communicate with them (Kisch 2004). However, two developments are worth noting that exposed some of these deaf signers to other sign languages and some Arabic literacy.

Soon after the Israeli military rule was lifted in the Negev (1966), Israel occupied the West Bank (during the 1967 war). Negev Bedouin could renew contacts with relatives in the West Bank and Jordan and new contacts were established. Consequently, four deaf siblings (cluster IV fig. 2) spent a year in a school for deaf students in Halhul. Their father had married a third wife from this West Bank Palestinian town, and her family hosted his deaf children during one school year. While their schooling did not last long, they acquired some basic literacy in Arabic and were exposed to signing that was most likely related to Jordanian Sign Language (LIU). While it is difficult to determine what impact this exposure had on their language development, it does further demonstrate that is not the case that this generation had no contact with other deaf people or sign languages.

Additionally, at least three men of this generation had as young adults attended occasional activities organised by the Deaf club in the nearby district town of Beersheba, which was established by Jewish-Israeli deaf women and men, and is associated with the national Association of the Deaf in Israel. One of them also visited other deaf clubs in the north of the country. Another recounted several mutual visits with a deaf man he had befriended from a Jewish town. While these contacts were not long-lasting they did provide these men with some exposure to ISL, as did television, which started to appear in households only after most of this generation reached adulthood.

Several of the deaf signers in this generation have younger siblings who were not included here; practically all deafAl-Sayyid born after 1970 attended school, which drastically changed their sociolinguistic environments. 


\subsubsection{Third generation signers [Niv, Hebrew school for the deaf]}

This generation of signers can also be labeled the Niv (school) generation; they are distinguished from the earlier generations of deaf signers by systematic exposure to signed instruction in school, including few deaf staff members.

Of the 36 third generation signers, all but two attended the Niv school. And all but one of the men in this group spent an additional 2-4 years at a program for deaf students in the Onim vocational boarding school. Most special education schools for the deaf in Israel generally take the total communication approach using (written and signed) Hebrew as the language of instruction, and some ISL (Weisel \& Zandberg 2002). Both Niv and Onim generally take such a total communication approach and employed at least one deaf instructor.

However, third generation signers are marked not only by their exposure to formal instruction but also by being schooled separately from their hearing peers. For years they rose early in the morning, before their hearing siblings, to gather with their deaf relatives at the intersections of the dirt roads crossing the village to be bused to the nearby district capital of Beersheba.

Initially Niv was a mixed school with mostly Jewish students but gradually Bedouin students made up the majority of the school's population. It did however remain a school belonging to the Hebrew (Jewish) sector of the Israeli education system and thus for years it employed solely Jewish teachers and used content designed for Jewish students; until shortly before the closing of the school no Arabic was taught and the curriculum typically marked Jewish (rather than Muslim) holidays. The mostly hearing staff was generally trained to use total communication based on Hebrew and ISL.

This generation can be further grouped into two cohorts of signers; the later Niv cohort (3.2) are mostly bilingual (in ABSL and ISL) and often prefer communicating in ISL among themselves, while the early Niv cohort (3.1) vary in the degree of fluency achieved in ISL, and in the extent they continued to use ISL after graduation.

\section{Cohort 3.1: "Early Niv cohort"}

This cohort includes 10 deaf adults: all are married and all but 2 have children.

Three of the 10 grew up in households with older deaf siblings or a deaf parent.

Another is a half sibling of a second generation deaf signer. Thus they mostly did not grow up in homes with elder deaf signers; two grew up 
with a competent hearing signing parent, and the remaining signers in this generation had only more distant signing relatives, and no immediate deaf neighbours.

This cohort includes only one woman who never attended school and another who attended for one year only. The remaining individuals had between 5-12 years of schooling (with an average of nine years). Three of these early Niv graduates are bilingual and fluent ISL signers; they all participated in some additional programs or activities in ISL. The remaining signers in this cohort vary in the degree of fluency developed in ISL and they mostly did not continue to use ISL on a daily basis after graduating. They also differ in their literacy skills, but mostly do not have functional literacy.

While one could consider including the two unschooled women with the previous generation despite their years of birth, such a classification would have to be supported by careful analysis of the signing used in their parental households, in order not to overlook the signed input. It would be however unreasonable to include those schooled signers with their previous generation. Sandler et al (2005) include at least one of the more fluent ISL signers of this cohort in their initial sample (labeled second generation signers) despite the signer's clearly divergent linguistic output. In a later article the researchers report that in the 2005 study this young woman consistently produced clauses with SVO (the basic word order in Hebrew and spoken Arabic), rather than the SOV used by the other second generation informants, thus acknowledging possible influence from her contact with Arabic and Hebrew (Padden et al 2010: 394). This pattern confirms that, despite belonging to the same structural generation as her older (second generation) siblings, she is better classified with the next sociolinguistic generation, 3.1, as she is classified here), consistent with her age and schooling.

\section{Cohort 3.2 "Late Niv cohort"}

This cohort includes 26 young deaf adults born between 1981 and 1991. All but one ${ }^{15}$ are graduates of the Niv school. Unlike the early Niv cohort the majority of this cohort are fully bilingual and competent ISL signers. While many prefer to communicate in ISL among themselves, they are also all capable ABSL signers. As is the general figure for Al-Sayyid, slightly over $20 \%$ of this cohort have a deaf parent. Additionally some have older deaf siblings, meaning that roughly a third of this group grew up in households with older deaf signers. They all communicate with their hearing relatives in ABSL. 


\section{Shifra Kisch}

The eldest four men and more than half of the women of this cohort are married (like their hearing relatives, women are on average younger at marriage than men). Most notable however is the fact that more than half of these young married deaf women are married to deaf men who are not from Al-Sayyid, while all married deaf men of this cohort are married to hearing Al-Sayyid relatives. Later I will further discuss the sociolinguistic impact of the changing marriage patterns of this generation.

The Niv school was not the only source of exposure to ISL for this cohort. All of the men of this cohort received vocational training in the Onim residential school for 2-4 years. Most of the women participated (using ISL interpreters) in a vocational training program lasting several months provided by the rehabilitation center in Beersheva. Most of these young women also participated in an empowerment program organised by The Institute for the Advancement of Deaf Persons in Israel, led by a (Jewish) deaf instructor and a hearing Arab instructor trained as an ISL interpreter ${ }^{16}$.

Additionally this cohort was the first to have access to TVs from a younger age, providing additional exposure to ISL (and LIU) interpreted programs. Despite the fact that most of the village is still dependent on generator power and many in this cohort have only basic poor literacy skills many regularly use internet and mobile phones for video-calls, chat, SMSs and other written communication in Hebrew.

Those born in 1991 were the last to be admitted to the Niv school; the school was finally closed in $2005 .^{17}$

It might be the case that some individuals can be better grouped with the previous or succeeding cohort than their actual age group. This might be of particular relevance to some of those born in years that distinguish between different cohorts. For instance, one might consider grouping the only woman in the (3.1) early Niv cohort (among the oldest of the women in this cohort did not attend school at all with the previous second generation signers (2), as indicated by analysis of her kin and non-kin networks.

Another example would be the youngest of the late Niv cohort (3.2), born in 1991; she is the only member of this cohort who did not go to the Niv school but rather was the first to be schooled in Arabic and mostly attended the Andalus School, (see also f.n. 13).

\subsubsection{Fourth generation signers}

All those born from 1992 onwards are for now grouped in this fourth and youngest generation of deaf signers. Like the previously identified genera- 
tions, the birth years of this group too range over approximately 20 years. However, the infants among them are in their early phases of first language acquisition, and it is too early to conclude which sociolinguistic circumstances will shape their mature signing.

Only very few members of this fourth generation have had regular or direct communication with first generation deaf signers. They are all schooled in Arabic rather than Hebrew, and no longer attend the special schools for deaf children that brought together deaf Bedouin children from all over the Negev. In this respect they can be labeled the post-Niv generation.

They can be further grouped into two cohorts of signers: the first cohort (4.1) were the first to attend Arabic Bedouin schools, and went to the Andalus School in the nearby town of Tel-Sheva. The younger (4.2) cohort includes the first to be schooled in the local (Arabic) school in Al-Sayyid and benefits from instruction by deaf teaching assistants (all Al-Sayyid Niv graduates) from kindergarten onward. This is however not the case for a growing number of children in this cohort who are singled out as candidates for cochlear implants or oral education. This cohort is thus increasingly subject to segregation based on their audiological status.

Cohort 4.1 Andalus, classes for deaf students in Arabic schools.

Like the older Niv generation these students too were daily bused out of the village for schooling. But unlike Niv students, they were the first cohort to attend classes in Arabic schools.

In 1996 the Ministry of Education ${ }^{18}$, displease with the primarily Bedouin population of this (Hebrew) school, decided to gradually close the Niv School for the Deaf. The 2003 mainstreaming act and growing pressure from Bedouin parents concerned with their deaf children's exclusively Hebrew education and poor outcomes, further reinforced this decision. By the time the Niv School was finally closed (2005) deaf Bedouin students were enrolled in classes for deaf students in seven different Arab schools throughout the Negev.

All the Al-Sayyid deaf students born from 1992 to 1999 were enrolled in classes for deaf students in the Andalus School in the Bedouin town of Tel-Sheva. Here they mostly received instruction from inexperienced and untrained hearing teachers who used irregular and inconsistent ISL vocabulary to codify written and spoken Arabic. While the staff were Arabicspeaking and thus more familiar with their students' socio-cultural background, they received little to no specialised training and none had any 


\section{Shifra Kisch}

experience instructing deaf students. Two deaf assistants (both non Al-Sayyid Bedouin graduates of the Niv School) were employed in the Andalus School (not simultaneously).

After completing primary school at the Andalus School most female students in this cohort began attending classes in other towns, where there were no deaf staff. Most male students proceeded to the Onim vocational boarding school, with its signed program for deaf students.

The population of deaf students in the Andalus School grew rapidly; deaf students from all major Bedouin groups with high incidences of deafness were assigned to this school, and together they constituted roughly a third of the deaf students. Catering to the needs of this rapidly growing group of deaf students soon became a burden for the inexperienced staff. In this context the proposal to open deaf classes at the Al-Sayyid school was finally approved. In 2010 most of the remaining Al-Sayyid students from this cohort were relocated to one of the schools in Al-Sayyid.

The ISL signing of Andalus students is considered "different" by many of the younger Niv (School) cohort signers. While some Niv cohort signers use less neutral terms to describe this variation, such as "broken" and "inaccurate," they do recognise it as ISL. I have recorded several persistent sign variants commonly used by Andalus students, all based on ISL (mostly with variations in hand shape or movement) ${ }^{19}$.

\section{Cohort 4.2 Al-Sayyid school}

Practically none of the deaf children in this cohort have had any direct exposure to first generation signers, even though this group includes grandchildren and great-grandchildren of first generation signers. They do however enjoy increased interaction with hearing signing peers, as they share the same school grounds. Those born in 2000/1 were the first to attend the preschool class opened in Al-Sayyid in 2006. By now more than half of the children in this cohort attend such classes.

From the very start each of these classes had a deaf Al-Sayyid teaching assistant from the late Niv cohort (3.2). Notwithstanding their lower rank (as mostly untrained teaching assistants) the role these deaf instructors play in the education of their students cannot be overrated; not only do they translate the (hearing) teachers' intentions, in several cases they essentially became the primary instructor due to the main instructor's inadequate training. During breaks the deaf assistants often gather and provide a signing environment. As noted above, this cohort (3.2) has a clear preference for using ISL 
to communicate with one another. They do use local signs on school grounds when communicating with hearing students and local staff. However, they insist on using ISL when communicating with their deaf students or with the mostly non Al-Sayyid ${ }^{20}$ teachers of deaf students.

These young deaf assistants make a clear distinction between "school signs (ISL)" and "local signs" and see it as their duty to teach their young students (4.2) ISL. In interviews many have emphasised that ISL will enhance their students' communication beyond the local community and allow them to utilise various services. They point out that unlike themselves and the 4.1 cohort, the members of the Al-Sayyid (4.2) cohort will encounter non Al-Sayyid deaf students later in their educational trajectory. While in this respect the young instructors appear to favor the Niv School compared with the new educational setting, they are more often ambivalent, pointing out the various advantages and disadvantages of each setting.

Students of the Al-Sayyid classes enjoy better communication on school grounds than did the Andalus students, despite the fact that their teachers are non Al-Sayyid, untrained, and inexperienced, as was the case at the Andalus School. However, in the Al-Sayyid classes, in addition to their deaf instructors, they are surrounded by hearing siblings and relatives among staff and peers, many of whom use local signs.

Half of the 50 deaf children in this cohort are attending primary school (including kindergarten classes) in Al-Sayyid. One of the classes is reserved for implanted children, and several children are "mainstreamed" in hearing classes ( 9 of the 14 implanted children are enrolled in supposedly non-signing classes). Some younger children in this cohort are enrolled in daily oral preschool programs for deaf children outside of Al-Sayyid or attend weekly sessions at such programs ${ }^{21}$. Accordingly, the students in this cohort are increasingly subject to segregation and classification according to audiological criteria and oral expectations. While it is becoming increasingly common for deaf children to receive some oral training, systematic oral training in articulation or speech reading is mostly restricted to Cochlear Implant (CI) candidates or implanted children. However, few of these implanted children can actually rely on oral input for instruction. Consequently instructors as well as parents may produce a rather irregular, often denied form of signed communication.

Thus I conclude by repeating the earlier mentioned indefinite classification of this later cohort. The youngest children of this cohort are infants in the early stages of language acquisition. It is too early to conclude what shared sociolinguistic circumstance will shape their future linguistic development. Hence, this relatively large group of roughly 80 youngsters might 
prove to include signers who will have more in common with the succeeding fifth generation of Al-Sayyid signers.

\section{The Major Factors Transforming The Al-Sayyid Sociolinguistic Landscape}

\subsection{Bi/multilingualism and Schooling}

One of the most obvious manifestations of the transforming sociolinguistic landscape among the Al-Sayyid over the last 30 years is the widespread bi/ multilingualism among both deaf and hearing (see fig. 3). The increase in literacy rates is another significant yet unequal sociolinguistic development, with deaf students having relatively poor literacy skills compared to their hearing counterparts. Primarily the result of separate schooling, increases in bilingualism and literacy are accompanied by the shrinking of the social space shared by deaf and hearing. Not only did they attend separate schools, the different social networks and social resources obtained through schooling result in different life trajectories and opportunities for deaf and hearing. For example, so far deaf people have no access to higher education nor a place among the related emerging class of Bedouin young professionals. At the same time, the employment of several deaf women as teaching assistants at local schools results in their slightly higher employment rate compared with their hearing sisters. Increased participation in social activities reserved for deaf people as well as the more recent deaf-deaf marriages of several deaf women all mark a new "sense of Deafhood" (Ladd 2003) and deaf sociality emerging among deaf Al-Sayyid and other Negev Bedouin (Kisch 2007).

Largely mediated by the introduction of deaf schooling, changes in both labour and marriage patterns generally correspond to the grouping of the deaf signers presented in the previous section. Towards the end of this section I will review the major differences in these respects between generations of signers and their sociolinguistic implications.

Despite the differences between deaf and hearing, men and women, the members of the Al-Sayyid shared signing community regularly move between languages; primarily Arabic, Hebrew, the local Al-Sayyid Bedouin Sign Language and Israeli Sign Language. Accordingly, code switching and mixing are increasingly common. Hearing signers often switch between speech and sign, or sign their Arabic. Deaf signers who prefer to communicate in ISL with their peers (those in cohorts 3.2, 4.1, and some from 4.2) increasingly use ISL with (schooled) deaf relatives in home settings where 
they otherwise communicate in ABSL. Deaf children in the youngest cohort who do not have immediate deaf relatives have most often been observed to introduce ISL signs to hearing relatives at home. Both result in common code-switching and mixing ${ }^{22}$.

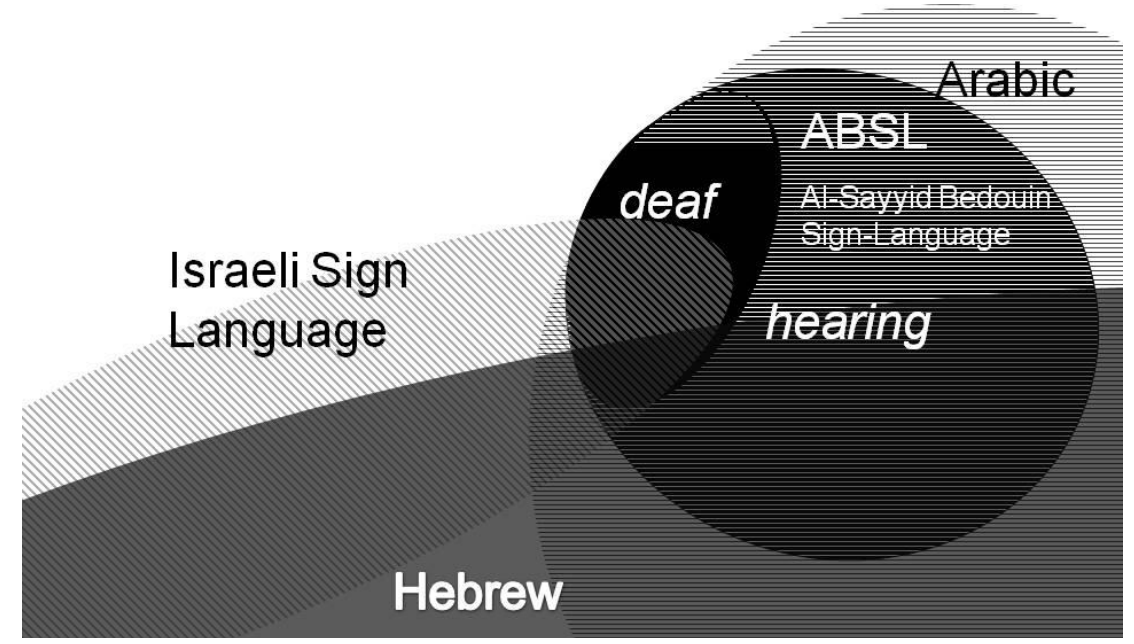

Figure 3. Bi/Multilingualism among deaf and hearing Al-Sayyid signers.

Hearing signers' bilingualism in the local spoken and signed languages (bimodal bilingualism, Emmory et al 2008) is one of the notable attributes of shared signing communities. With the introduction of schooling, increased access to media, and increased participation of hearing men in the labour market, unimodal bilingualism also gradually became more common for both deaf (ABSL and ISL) and hearing (spoken Arabic and Hebrew) people. Deaf people were introduced to the national sign language through schooling, but also through electronic media (such as signed interpretation on TV and more recently also virtual social networks with video communication). When literacy is considered as an additional modality (c.f. Grosjean 2010), deaf people are also increasingly becoming bimodal bilinguals, as literacy introduced deaf people to a new language (Hebrew or Arabic). Yet, not all Niv students have functional literacy skills; others have relatively poor skills ${ }^{23}$ but regularly use Hebrew vocabulary for SMS (Short Message Service).

While the social space shared by deaf and hearing is generally accommodating for deaf people in shared signing communities, social structures and practices should be examined for how they may both facilitate and restrict deaf people (Kisch 2007, 2008). Even though separate deaf schooling and new marriage and work patterns have reduced the social space shared by 


\section{Shifra Kisch}

deaf and hearing, these processes also allow deaf people to gain valuable resources (such as an education, some literacy, a vocation, work and income, and new social networks) to better their lives and opportunities.

\subsection{Changing marriage and labour patterns}

Finally I will review the major differences in marriage and labour patterns between the previously demarcated Al-Sayyid generations of signers, and their sociolinguistic implications.

For the first generation of deaf and hearing signers, marriage across the Al-Sayyid lineages was crucial to exposing a greater number of hearing relatives to frequent signed communication in the then-emerging signing system. All Al-Sayyid lineages included second generation (deaf or hearing) signers. All the deaf signers of this generation married hearing partners ${ }^{24}$, and half of them married across the lineages. This further contributed to the spread of signed communication and the increase of fluent hearing signers.

The more recent occurrence of deaf-deaf marriages of third generation (3.2) brides, have quite a different sociolinguistic effect, enhancing the use of and preference for ISL. Generally, deaf-deaf marriages involve the migration of deaf women from the Al-Sayyid settlement to neighbouring, even distant, locations - reducing their own as well as their children's interaction with both deaf and hearing local signers. Most of these brides gradually adjust to their primarily hearing in-laws' homesigns but communicate in ISL with their husbands.

With one exception, deaf-deaf marriages are restricted to women of the (3.2) cohort who are married to non-Al-Sayyid deaf husbands: one second generation deaf man married a much younger third generation deaf woman as a second wife. They constitute the first and - so far, only - local Al-Sayyid deaf-deaf couple. While they generally communicate in ABSL, the young deaf bride encourages her husband to learn ISL and to use it with their infant deaf daughter. At least three third generation deaf Al-Sayyid men considered finding a deaf partner but ended up marrying hearing relatives. For most of the partners involved, deaf-deaf marriages constitute a new form of deaf sociality corresponding to an emerging and gendered sense of Deafhood among the Negev Bedouin (Kisch, 2007).

The marriage and residential patterns of hearing signers are equally important for tracing the avenues of linguistic transmission and variation. While most men and women remain in the village after marriage, women 
rarely remain in the same residential compounds or clusters, as many marry across the major Al-Sayyid lineages. Thus, concluding that there are strong bonds within but not across families (Goldin-Meadow 2005:2271), is inaccurate.

\subsection{Labour and social networks}

Lastly, generations of signers also differ in the labour patterns that inform their sociolinguistic networks. Mostly, Bedouin (men) inhabit the lower strata of the Israeli labour market. Negev Bedouin's unemployment rates are among the highest in the country (Marx 2000, Jakubowska 2000, Abu-Rabia 2000). Thus, finding work does not only generate social networks but almost entirely depends on them.

Among the Negev Bedouin, the shift from household production to wage labour introduced new inequalities and social gaps. Thus while a class of Bedouin young professionals is emerging, the Bedouin labour pool is still mostly made up of unskilled men with primary school education.

In the era that corresponds to the adult lives of first generation deaf signers, Bedouin were largely kept out of the labour market (Marx 1967), and wage labour was rare. Also farming and animal husbandry- previously the dominant sources of livelihood- were then limited by the restrictions of military rule. Mostly unskilled wage labour started to occur in the next generation (corresponding to second generation signers), but labour was still largely organised through family networks. Most common were families pooling for seasonal agricultural labour; later family cooperations among the Al-Sayyid often involved father and sons, or several brothers sharing the contracting of heavy equipment. In the age group of third generation signers, increasingly more men work in occasional or more stable manual-labour jobs. Yet, kin-based networks often remain important in finding work. For many work involves weekly commuting to the center of the country, sharing lodgings with co-workers and relatives.

It was also during this generation that the already existing occupational differentiation among the Negev Bedouin grew significantly. Among the Al-Sayyid of this generation sources of income are as diverse as: seasonal agricultural workers, watchmen on construction sites, construction workers and several successful building contractors, tractor and truck drivers and several owners, and mechanics' assistants and teachers. While many keep some livestock for domestic use, only very few among the Al- Sayyid maintain larger herds for profit. 


\section{Shifra Kisch}

Increasingly, deaf men from the third generation of signers found employment mediated by their vocational school and their non-kin deaf social network. Vocational training mostly provided them with skills such as welding, assistant mechanics or cooking. However they also entered the labour market at a time of a general slowdown in the national economy and a sharp decline in the number of gainfully employed Bedouin. Despite the fact than more young deaf men have at least some vocational training, the recent recession and further rise in already exceptionally high unemployment rates has left many hearing and most deaf men unemployed and dependent on welfare benefits and occasional informal day work.

Only a small portion of Bedouin women participate in the labour market; those who do are mostly employed as trained and untrained educational staff at the local schools, as is the case for most employed hearing young women among the Al-Sayyid. Employment rates among young deaf women are even slightly higher than those of hearing women of their age.

With some vocational training but poor literacy, deaf men and women occupy the middle range of occupations. Disparities between deaf and hearing are mainly manifested in the ease of access to a (limited) number of vocational training opportunities and the lack of access to higher education and consequently to the highest strata of the labour market ${ }^{25}$. These shifts in the structure of labour have had several sociolinguistic consequences.

Work in the community or through kin-based networks and cooperation enhanced the use of local sign language and the social space shared by deaf and hearing signers. The individual integration of deaf people in the labour market was assisted by some degree of literacy in Hebrew for basic workrelated communication, and often did not enhance any signed communication other than some ad hoc gesturing. Joint employment of deaf young men (commuting and sharing lodging) is mostly an extension of their school networks and enhanced their daily use of ISL.

Deaf women's employment as assistants at the local schools enhanced both their communication with hearing relatives in ABSL as well as their use of ISL with fellow deaf assistants and their students. Their lack of training in Arabic (all assistants so far are of the 3.2 Niv cohort) had occasionally been used to question their qualification for the job. However, their employment has generally contributed to their own status as well as to the acknowledgement of the value of signed communication. Most significantly it has contributed to the shaping of their young students' sociolinguistic environment. 


\section{Conclusion}

Based on analysis of kin and non-kin social networks, the major shifts in the sociolinguistic settings, and the actual observed communication practices of deaf and hearing signers, this paper identifies four generations of Al-Sayyid deaf signers (including 4 sub cohorts). Hearing signers can generally be classified in accordance with the deaf relatives of their age group. However, it is equally important to examine their social networks and establish which signing relatives and individuals they communicated with regularly as indicated by the recording of genealogies, residential patterns, observations and interviews.

The availability and range of signed input is the major factor distinguishing generations of signers. The primary factor distinguishing first and second generation signers was the absence of adult models for the first generation versus the second generation signers' exposure to an emerging sign system and adult (hearing or deaf) signers. As the community grew, births of deaf people were increasingly spread throughout the community. Homesigns have most likely evolved in the hearing families of several second generation signers, inspired by hearing signers who had some exposure to the earlier signing of relatives. Hearing signers soon outnumbered deaf ones and play an important role in the development of the local signing system and in its maintenance and intergenerational transmission.

Besides tracing avenues of intergenerational transmission I underscore the exposure and input from other languages. Here, as is the case for all shared sign languages, the spoken language of all hearing signers is most obvious. Moreover, in the case of Al-Sayyid, deaf signers are exposed to other sign languages and are increasingly bilingual. For first and second generation signers, encounters with other sign languages occurred only in their teens or later and thus interference might be limited. Yet, it is not the case, as concluded, for example, by Aronoff and colleagues (2005), that the first two generations had no contact with other deaf people. Once formal deaf education was introduced, exposure to ISL and to written language (Hebrew or Arabic) could no longer be ignored when defining criteria to distinguish between second and third generation signers. Thus, I urge meticulous consideration of the potential influences of such factors in demarcating the generations that are compared for the purpose of detecting variation in linguistic properties. 


\section{Shifra Kisch}

Regardless of the linguistic skills developed in those languages, exposure to other languages cannot be dismissed or rendered negligible. Even in the absence of fluency or full bilingualism, it has been suggested that very partial linguistic knowledge held by only a few of those involved in the emergence of a novel language may have an influence (Arbib 2009). It would thus be remarkable if widespread (signed, spoken and bimodal) bilingualism did not leave recognizable marks on the development of $\mathrm{ABSL}^{26}$. Before such influences can be persuasively ruled out, it is essential to first group signers in accordance with their different linguistic inputs and sociolinguistic settings. In other words, only careful prior consideration of potential linguistic models and interfaces would corroborate claims regarding their insignificance. Padden et al (2010) briefly mention finding support for the effect of schooling on signers' word order in ABSL. Yet, most of the publications on $\mathrm{ABSL}^{27}$ overlook widespread bilingualism and schooling among Al-Sayyid signers.

The most prominent sociolinguistic factor distinguishing second and third generation (deaf) signers among the Al-Sayyid is access to education. The four cohorts of the third and fourth generation deaf signers are primarily distinguished by their increased and partially formalised linguistic exposure and their social networks resulting from different educational settings. Schooling in general introduced students to a new signing system, and at least some literacy. Additionally, it introduced deaf students to an additional signing (student) community and reduced the social space shared by deaf and hearing. Consequently, deaf schooling transformed the Al-Sayyid sociolinguistic landscape for both deaf and hearing.

Only 14 out of the total of 134 deaf descendants among all generations of Al-Sayyid have not experienced some form of schooling. Two aspects of schooling need to be considered: the exposure to the language used for instruction, and the way schooling shaped students' sociolinguistic networks. These social networks inspired new labour and marriage patterns among deaf people and enhanced the prestige attached to ISL. These factors contribute to students' language development and their use of ISL as much as, if not more than, the official instruction.

In other shared signing communities social factors might interact differently in shaping the sociolinguistic landscape. Factors might not correlate as neatly as Al-Sayyid age groups correspond to school attendance. Additionally, social factors might not be as markedly interrelated; among the Al-Sayyid major shifts in both labour and marriage patterns of deaf men and women are mediated foremost by schooling. In Adamorobe (Ghana), employment is strongly related to migration but few deaf people engage in 
it (Nyst, 2007; Kusters 2011). In Desa Kolok (Bali), deaf men and some deaf women commonly participate in several deaf labour teams, performing distinctive tasks in the village and beyond (Marsaja 2008; de Vos, this volume). Such employment patterns create social space and roles reserved for deaf people. In addition, in Desa Kolok, deaf-deaf marriages occurred early on in the history of the community (Marsaja 2008) unmediated by deaf schooling.

Many social factors have gendered effects, differently shaping men's and women's networks. This is most obviously the case for marriage in both Adamorobe and Al-Sayyid though in utterly different ways. In Adamorobe marriage options are more severely limited for deaf men (Kusters 2011; this volume), possibly restricting their social network and the intergenerational transition of AdaSL. In Al-Sayyid while married men mostly remain in the same residential environment, married women move to a new environment within the village, and more recently away from the village to be wed to deaf partners with whom they communicate in ISL. Also work and schooling differently inform the social networks of Bedouin (deaf and hearing) men and women.

Many more differences exist between these communities (see Kisch 2008; Nyst in press) but these few examples indicate that the sociolinguistic impact or relevance of social factors may vary significantly. My point is that exploring social networks remains a key factor in recognizing the factors transforming sociolinguistic landscapes (c.f. Milroy 1980). Furthermore, when variation in sociolinguistic networks does not correspond to agerelated factors there is no reason to assume variation in signing is solely -or even primarily- along generational lines. All possible avenues for language exposure, spread, and transmission should be considered

For shared sign languages that linguists may consider to be emerging, tracing kinship and descent is essential for the identification of the first assumed signers, and for revealing possible avenues for the intergenerational transmission of their linguistic practices. In studies of shared sign languages that are no longer traceable to their first users, identifying sociolinguistic generations of signers might be less consequential. Nevertheless, given the often prominent role of kinship in shaping social relations and language acquisition, tracing kinship and descent can be instructive in understanding the social and sociolinguistic setting and history. In both cases it remains important to recognise that kinship charts do not represent actual social relations. Moreover, as descent-groups grow their genealogies become increasingly convoluted and ambiguous. It is thus essential to distinguish between structural generations of descent (typically represented in kinship 


\section{Shifra Kisch}

diagrams) and generations of actual contemporaries assumed to have shared socio-cultural experiences. Next the shared circumstances they were actually subject to need to be identified, and their potential sociolinguistic effects analysed. When kinship is assumed to be a dominant factor in shaping social relations, it can be an even greater source of confusion on both the emic and etic levels. Despite cultural idioms, kinship is seldom the only source for networks of affiliation and collaboration. Based on my data, I illustrate the importance of complementing kinship diagrams with an analysis of actual (kin and non-kin) social relations. I show how critical it is to situate such information in its social and historical context, and to consider intra-generational age gaps, residential patterns and actual social relations whenever genealogies are recorded in order to reconstruct the language environment, contemporaries and adult models of past generations.

Finally, to return to the Al-Sayyid shared sign language, bi/multilingualism is characteristic of its sociolinguistic landscape. Deaf and hearing Al-Sayyid signers are embedded in several diverse language communities. Bilingualism and preference for ISL should not only be acknowledged for the sake of recognizing (or refuting) possible influences on ABSL. From an anthropological perspective, it is equally important to recognise how these practices may transform social relations. Among the Al-Sayyid -as is often distinctive to shared signing communities - signing is not used only by deaf people to communicate among themselves. Rather signing is what deaf and hearing people do to communicate. This remains the case for the use of the local sign language (ABSL). Yet, the use of Israeli Sign Language (ISL) is increasingly becoming an instrument of deafness, and something not as readily shared between deaf and hearing people. Thus ISL is emerging as a marker of deaf sociality; it is what young deaf people use among themselves. For younger generations, ABSL might increasingly become the language largely reserved for communicating with hearing people. Such developments both underscore the shared nature of such sign languages and further indicate the shifting boundaries of the Al-Sayyid shared signing community. Ethnographically sound methods reveal that even when the distinctiveness of community boundaries seem apparent to its members and to outside observers, it is important to remember that networks of relationships and 
related sociolinguistic practices have far less distinct boundaries.

\section{Notes}

1. I here avoid the term "village sign language" (Zeshan 2006; Meir et al 2010a); from an anthropological perspective the term "village" is of little descriptive or analytical value.

2. Not all shared sign languages are considered young or emerging languages. Several are no longer traceable to their first users (see Marsaja 2008 for Kata Kolok, Bali; and Nyst 2007 for AdaSL, Ghana). Other cases have been observed to be endangered (Nonaka 2009).

3. The challenge in this case is how to batch the cohorts into generations of signing. Some critics have questioned Senghas et al's (2004) choice of 1984 and 1993 as demarcation line between these cohorts of signers.

4. They define the first generation in terms of structural generations "The first generation in which deafness appeared in the community (the fifth since the founding of the community)" Sandler at al 2005:2662; Aronoff et al 2005:31), while second generation is defined in terms of a general age range (in their 30 and $40 \mathrm{~s}$ one in her 20s), and third generation in the most general terms (teenagers and children). Meir et al 2010b:310 "second generation signers (in their 40s) and four third generation signers (in their teens and early twenties)". In some cases generations are not defined at all (Sandler et al 2011).

5. My native language is Dutch, but I received my high school education in Israel, and my Hebrew education program included classical Arabic as second language.

6. In contrast to the 5th generation reported by Aronoff et al (2004), (2008); Meir (2010); Meir et al (2010a); Sandler et al (2011).

7. Exact figures vary depending on definitions of consanguinity, ranging from first degree cousins to all but non-relatives (e.g. Weitzman 2000; VardiSaliternik et al 2002; Zlotogora et al 2009; The Galilee Society 2011).

8. For other forms of skewed diagrams see Tjon Sie Fat $(1983,1990)$

9. I am grateful to Prof. Tjon Sie Fat for kindly offering to compose these digital kinship diagrams, meticulously transforming several versions of my drawings.

10. It is also reported to be the case for Algerian Jewish Sign Language ( see Lanesman and Meir, this volume)

11. Emmorey et al (2008) have introduced the term code-blending to refer to such simultaneous speech-sign production, found to be common among bimodal bilinguals blending English and American Sign Language.

12. These last 2 are non-signing individuals who independently developed intelligible speech previous to school intervention or oral training and are 


\section{Shifra Kisch}

considered "hard of hearing" or HALF DEAF by both deaf and hearing members of the community. They are however relevant for the calculation of the rate of congenital hearing loss.

13. As mentioned above, four of the initial Al-Sayyid cousin-marriage unions bore 9 deaf descendants among Al-Sayyid's 4th structural generation of descent, however given the age gaps and overlaps, the youngest was born when Hadra was already 44 years old and a mother of 7 . Furthermore, within this time span eight more deaf descendants were born. It is therefore best to frame this generation too by year of birth, rather than following structural generations of descent.

14. Hadra's father ran a British mandatory supply store and one of his deaf sons who assisted him in the store was at least partially literate. At the time most of his (deaf or hearing) peers could not read nor write.

15. This young woman was from a young age enrolled in Arabic schools. Her father, a teacher himself, was the first to be captivated by oral training and mainstreaming, only to be disenchanted later. His daughter (whose name sign alludes to her hearing aid) later joined her slightly younger peers (of the 4.1 cohort) at the Andalus school.

16. http://www.sela.org.il/index.php?page=הריסמי (in Hebrew) http://www.sela.org.il/\%d7\%a2\%d7\%a8\%d7\%91\%d7\%99\%d7\%aa / (in

Arabic). No English page available other than general site http://www.dpii.org/

17. The remaining students continued their studies in the Onim School and the last female students were relocated to the newly opened classes for deaf students in two high schools in Bedouin townships. Four of these female students from Al-Sayyid were aged 14-16 years old when the Niv school closed.

18. The Ministry of Education's 1996 resolution closing the school asserted that students born after 1990 would be referred to (at the time nonexistent) special classes in Arab schools. In fact, all but one of the students born in 1991 were still assigned to Niv.

19. At least one of these signs was introduced by one of the senior (hearing) teachers who taught sign language to her fellow teachers. Though a veteran teacher, she had only taken one basic ISL course herself.

20. These teachers are non-Bedouin Arab teachers or Bedouin from other Negev towns or villages. Roughly $40 \%$ of the teachers in Negev Bedouin schools are recruited from northern or central Israel where there is a surplus of Arab teachers (Abu Rabia 2000; Abu-Rabiyya et al 1996). Consequently those teaching in the Negev are often young and inexperienced and there is a high turnover rate.

21. The main program providing such training is the local branch of Micha, a rehabilitation center for preschool infants and toddlers. Although other Micha centers in Israel provide services in Arabic, Micha Beersheba was established in 1964 but did not have any Arabic speaking staff until 2007. Earlier only few Bedouin children were welcomed by this center, this has changed now 
that increasingly more Bedouin children have CIs and with the passing of the rehabilitative day care act. The center is known for its oral approach and strongly encourages CIs.

22. Another form of mixing, namely the mouthing of Hebrew or Arabic words or names, is uncommon in daily communication (with exceptions among children with CIs, the few deaf signers with significant residual hearing, and some less-fluent hearing signers).

23. The education available to deaf Negev Bedouin is comparable to the education available to their hearing Bedouin peers and to deaf Jewish students, that is to say, all groups fare worse than do hearing Jewish students .

24. Two of these men married hearing women from outside of the community.

25. This would include not only certified teachers and higher ranking educational staff but also more than a dozen (hearing) young men who have studied dentistry, medicine, pharmacology and law abroad.

26. As claimed by Aronoff et al 2005, 2008; Sandler et al 2005.

27. With the exception of Meir 2010 and Padden et al 2010.

\section{References}

Abu-Rabia, A.

$2000 \quad$ Employment and unemployment among Negev Bedouin. Nomadic Peoples 4(2). 84-93.

Abu-Rubiyya Salim, al-Athauna Fawzan, \& Salman al-Bador.

1996 Survey of Bedouin Schools in the Negev. Tel Aviv: ADVA center, Information on Equality and Social Justice in Israel.

Arbib, M.

2009 Invention and community in the emergence of language: Insights from new sign languages. In: Platek, S.M. \& T.K. Shackelford (eds.), Foundations in evolutionary cognitive neuroscience: Introduction to the discipline, 117-152. Cambridge: Cambridge University Press.

Aronoff, M., C. Padden, I. Meir, \& W. Sandler

2005 Morpological universals and the sign language type. In Geert Booij and Jaap van Marle (eds.), Yearbook of Morphology 2004, 19-40. Dordrecht: Kluwer Academic Publishers.

Aronoff, M., C. Padden, I. Meir, \& W. Sandler

2008 The roots of linguistic organization in a new language. Interaction Studies 9(1). 133-153 
Emmorey, K., H. B. Borinstein, R. Thompson \& T. H. Gollan

2008 Bimodal bilingualism. Bilingualism: Language and Cognition. 11(1). 43-61.

Falah, G.

1989 Welfare Geography of a Peripheralised National Minority: The case of Israel's Arab population. Urban Geography 20(5). $417-437$.

Fusellier-Souza, I.

2006 Emergence and development of sign languages: from a semiogenetic point of view. Sign Language Studies 7 (1). 30-56.

Goldin-Meadow, S.

2005 Watching language grow. Proceedings of the National Academy of Sciences of the United States of America 102 (7). 2271-2272.

Grosjean, F.

2010 Bilingualism, biculturalism, and deafness. International Journal of Bilingual Education and Bilingualism 13(2). 133-145.

Jakubowska, L.

2000 Finding Ways To Make A Living: Employment Among Negev Bedouin. Nomadic Peoples 4(2). 94-105.

Kegl, J., A . Senghas \& M. Coppola

1999 Creation through contact: Sign language emergence and sign language change in Nicaragua. In: De Graff, M. (ed.), Language Creation and Language Change: Creolization, Diachrony, and Development, 179-237.. Cambridge, MA: MIT Press.

Kisch, S.

2000 'Deaf discourse': the social construction of deafness in a Bedouin community. Department of Sociology and Anthropology, Tel Aviv University, unpublished MA. [in Hebrew].

Kisch, S.

2004 Negotiating (genetic) deafness in a Bedouin community. In Genetics, disability, and deafness. J. V. van Cleve (ed.), 148-173. Washington DC: Gallaudet University Press.

Kisch, S.

2007 Disablement Gender and Deafhood among the Negev ArabBedouin. Disability Studies Quarterly 27(4).

Kisch, S.

2008 The Social Construction of Deafness in a Bedouin Community in the Negev. Medical Anthropology, 27(3):283-313. 
Kusters, A.

2011 "Since time immemorial until the end of days": An ethnographic study of the production of deaf space in Adamorobe, Ghana. Unpublished dissertation, Centre for Deaf Studies, University of Bristol.

Ladd, P.

2003 Understanding Deaf Culture: In Search of Deafhood. Clevedon: Multilingual Matters.

Laura $\mathrm{P}$.

2005 The emergence of Deaf community in Nicaragua: With sign language you can learn so much. Washington DC: Gallaudet University Press.

Marsaja, I G.

2008 Desa Kolok - A deaf village and its sign language in Bali, Indonesia. Nijmegen: Ishara Press.

Marx, E.

1967 Bedouin of the Negev. Manchester: Manchester University Press.

Marx, E.

2000 Land and work: Negev Bedouin struggle with Israeli bureaucracies. Nomadic Peoples 4(2). 106-121.

Milroy, L.

1980 Language and social networks. University Park Press, Baltimore, MD.

Meir, I.

2010 The emergence of argument structure in two new sign languages. In M. Rappaport Hovav, E. Doron \& I. Sichel (eds.) Syntax, Lexical Semantics and Event Structure, 101-123. Oxford University Press. .

Meir, I. \& W. Sandler

2008 A language in space: The story of Israeli Sign Language. New York: Lawrence Erlbaum.

Meir, I., W. Sandler, C. Padden \& M. Aronoff

2010a Emerging Sign Languages. In M. Marschark and P. Spencer (Eds.) Oxford Handbook of Deaf Studies, Language, and Education, Volume 2, 267-280.. Oxford: Oxford University Press..

Meir, I., W. Sandler, C. Padden \& M. Aronoff

2010b Sign languages and compounding. In Sergio Scalise and Irene Vogel (eds.) Compounding. Amsterdam: John Benjamins. 


\section{Shifra Kisch}

Nonaka, Angela M.

2009 Estimating size, scope, and membership of the speech/ sign communities of undocumented indigenous/village sign languages: The Ban Khor case study. Language \& Communication 29(3). 210-229

Nyst, V.

2007 A descriptive analysis of Adamorobe Sign Language (Ghana). Utrecht: LOT publication.

in press Shared sign languages. in: Pfau, R., M. Steinbach \& B. Woll (eds.), Sign Language; An international handbook. HSK seriesHandbooks on Language and Communication. Berlin : Mouton de Gruyter.

Russo, T. \& V. Volterra.

2005 Comments on Children Creating Core Properties of Language: Evidence from an Emerging Sign Language in Nicaragua Science 309(5731):56.

Padden, C., I. Meir, W. Sandler \& M. Aronoff

2010 Against all expectations: Encoding subjects and objects in a new language. In D. Gerdts, J. Moore \& M. Polinsky, (eds.) Hypothesis A/Hypothesis B: Linguistic Explorations in Honor of David M. Perlmutter, 383-400. Cambridge, MA: MIT Press. .

Sandler, W.

2010 Language-specific phonetics without phonology. presentation at a colloquium of the Amsterdam Centre for Language and Communication Research, the University of Amsterdam. Tuesday, 14th December 2010.

Sandler W., I. Meir, C. Padden \& M. Aronoff

2005 The emergence of grammar in a new sign language, Proceedings of the National Academy of Sciences of the United States of America 102(7): 2661-2665.

Sandler, W., M.Aronoff, I. Meir \& C. Padden

2011 The gradual emergence of phonological form in a new language. Natural Language and Linguistic Theory 29. 503-543

Senghas, A.

2005 Language emergence: Clues from a new Bedouin sign language. Current Biology 15(12). 463-465.

Senghas, A., S. Kita \& A. Ozyurek

2004 Children creating core properties of language: evidence from an emerging sign language in Nicaragua. Science 305. 1779-1782. 
Supalla, J. S.

1991 Manually Coded English: The modality question in signed language development. In P. Siple \& S.D. Fischer (eds) Theoretical Issues in Sign Language Research Volume 2, 85-109: Psychology. Chicago: University of Chicago Press.

The Galilee Society

2011 The Palestinians in Israel, third socioeconomic survey 2010. Shefa-Amr: The Galilee Society.

Tjon Sie Fat, F.

1983 Age metrics and twisted cylinders: Predictions from a structural model. American Ethnologist 10.585-604.

Tjon Sie Fat, F.

1990 Representing kinship: Simple models of elementary structures. Leiden: Faculty of Social Science. (Unpublished $\mathrm{PhD}$ dissertation).

Vardi-Saliternik, R., Y. Friedlander \& T. Cohen

2002 Consanguinity in a population sample of Israeli Muslim Arabs, Christian Arabs and Druze. Annals of Human Biology 29(4). 422-431

Weisel, A., \& S. Zandberg

2002 Hearing impairment: Education of deaf and hard of hearing students. Tel Aviv: Open University (Unit 8). [in Hebrew].

Weitzman D., I. Shoham-Vardi, K. ElBedour, I. Belmaker, Y. Siton \& R. Carmi.

2000 Factors affecting utilization of prenatal testing for fetal abnormalities in a traditional society. Community Genetics. 3(2). 45-92.

Zeshan, Ulrike (ed.).

2006 Interrogative constructions in signed languages. Language 80 (1). 7-39

Zlotogora J, R. Carmi, B. Lev \& S. A. Shalev

2009 A targeted population carrier screening program for severe and frequent genetic diseases in Israel. European Journal of Human Genetics 17. 591-597. 


\title{
The Kata Kolok perfective in child signing: Coordination of manual and non-manual components ${ }^{1}$
}

\author{
Connie de Vos
}

\section{The acquisition of multi-channelled syntactic structures}

In spontaneous language use, signers and speakers alike recruit multiple articulators such as the hands, body, and face to produce composite utterances (McNeill 2000; Kendon 2004; Enfield 2009). Moreover, in the case of sign languages, facial expressions, head and shoulder positions and other non-manual signals are fully integrated into the grammar alongside manual components (Sandler \& Lillo-Martin 2006). As a result, many syntactic constructions require the coordination of multiple articulators. For example, in the expression of questions, both manual wh-signs and furrowed brows indicate content questions in a number of sign languages, see for instance Baker-Shenk (1983) on American Sign Language; Bergman (1984) on Swedish Sign Language, and Coerts (1992) on Sign Language of the Netherlands. Similarly, a multitude of sign languages marks negation by using manual and non-manual forms in concert (Zeshan 2004). This paper revolves around the acquisition of one such simultaneous structure: the syntactic marking of perfective aspect. In Kata Kolok - a village sign language of Bali, the full form of the perfective particle is produced with two 5-hands rapidly turning palm upward along with a lip smack glossed as 'pah'.

The existence of multi-channelled syntactic structures poses unique challenges to the acquisition of sign languages. In particular, children acquiring sign languages need to learn to coordinate manual and non-manual forms in order to produce well-formed utterances (Reilly \& Anderson 2002). One methodological issue in this story is that many non-manual forms find their origins in the co-speech gestures also used by the wider hearing community. Deaf children can therefore produce communicatively valid forms, which are also used by hearing individuals, but which are not fully integrated into their linguistic system. For instance, Anderson \& Reilly (1997) found that, while deaf and hearing children use negative headshakes by the one year mark, deaf children do not start to use manual negative forms until 18-20 months. 


\section{Connie de Vos}

Moreover, this manual form is not accompanied by non-manual marking until one to eight months after its first occurrence. Anderson \& Reilly interpret this first co-occurrence as evidence for a reanalysis of the gestural negative headshake, which is common to both the co-speech gestures of American English speakers and American Sign Language users, as part of linguistic negation within American Sign Language.

In the production of $w h$-questions, too, children do not initially use the appropriate facial expression, resulting in utterances that are ungrammatical in adult users of American Sign Language. Specifically, Reilly \& McIntire (1991) showed that, although toddlers use furrowed eyebrows to express puzzlement, deaf children initially produce $w h$-questions solely through manual signs (e.g., WHAT, WHERE) with no accompanying facial expression. The appropriate non-manual marking, furrowed brows and a head tilt, is on average delayed by more than one year. Based on these and other studies, Reilly (2006) generalises that in the acquisition of multi-channelled linguistic structures, the use of the manual forms precedes the use of coordinated non-manual morphology. She does not indicate however, which mechanism may underlie this observation. In my view, the delayed use of non-manual marking of $w h$-questions may be related to the input that deaf children receive through child-directed signing. That is, Reilly and Bellugi (1996) found that in more than $90 \%$ of the wh-questions that were signed to toddlers younger than 2 years old, deaf mothers did not use the standard furrowed eyebrows. According to Reilly and Bellugi, deaf mothers might have chosen to use neutral and raised eyebrow position as an alternative because furrowed eyebrows are associated with anger. All in all, the omission of potentially confusing non-manual marking in child-directed signing might explain the delay in the acquisition of such features by deaf children acquiring American Sign Language.

The studies on the acquisition of coordinated manual and non-manual forms in American Sign Language leave a number of questions unaddressed. It is conceivable, as state above, that the delayed acquisition of these simultaneous structures could have resulted from the input that these children received, that is to say, from the specific characteristics of child-directed American Sign Language. Alternatively, the coordination and integration of non-manual morphology may be mediated by the acquisition of manual forms more generally, in which case patterns are expect to hold across diverse structures and sign languages. This paper addresses this issue by looking at the distribution of non-manual and manual forms of perfective aspect in spontaneous signing produced by a deaf child acquiring Kata Kolok. As described above, the Kata Kolok perfective is essentially a coordinate 
manual/non-manual structure that combines a lip smack with one or two 5-hands rapidly turning palm upward. ${ }^{2}$ In adult Kata Kolok, this combined form (FINISH\#pah) is highly salient both in terms of frequency and through its use in greeting practices. The non-manual form 'pah' may also function as a bound morpheme that combines with lexical predicates and pointing signs, but this latter usage is marginal in the adult Kata Kolok corpus.

Aspect is possibly one of the most thoroughly researched areas of language development and its acquisition interacts with many linguistic factors. The overview presented below is primarily based on van Hout (forthcoming). The most prominent interaction is probably with lexical aspect, defined by the semantics of the predicate. One such semantic dimension is whether the predicate describes a telic event - an accomplishment or achievement, or an atelic event - a state or process. A typical atelic predicate is 'sleep', and good example of a telic predicate is 'wake up'. Corpus studies have indicated that children use aspectual markers at an early age, but that they initially produce perfective markers with telic predicates, and imperfective markers with atelic ones, presumably because of their semantic affinity. These findings indicate that children may not have fully grasped the meaning of these grammatical markers independent of the predicate. Furthermore, a multitude of studies have indicated that, while children may exhibit adult production patterns, their comprehension may be severely delayed (van Hout forthcoming). Languages mark aspect in different ways, including: verb morphology, analytical constructions, and particles (van Hout forthcoming). As mentioned above, Kata Kolok marks perfective aspect by the particle FINISH\#pah, and it is similar in this respect to Mandarin Chinese. Li \& Bowerman (1998) showed that in this language, the comprehension of aspect does not reach adult levels until the age of 5 . The child under consideration in this study is 24-36 months, and for these and other reasons, the present paper is inherently limited in its generalizations and essentially constitutes a pilot study. Taking this cautionary note into account, the paper represents the first developmental study in a village sign language and raises a few new issues as such.

The structure of this paper is straightforward. Section 2 presents a brief overview of the sociolinguistic facts that are most relevant to the acquisition of Kata Kolok. Before providing a grammatical analysis and examples of perfective aspect in section 4, section 3 describes the Kata Kolok corpus and the linguistic fieldwork on which this description is based. Section 5 explores the distribution of manual and non-manual perfective forms produced by a deaf child acquiring Kata Kolok. The conclusion, Section 6, emphasises how the comparative acquisition of sign languages, in particular of typologi- 
cally different ones, could considerably advance our understanding of the modality-specific and language-specific aspects of first language acquisition.

\section{Growing up in Bengkala: a deaf village in Bali}

Kata Kolok is a sign language that is indigenous to a village community of Bali, which has had a high incidence of deafness due to a recessive gene that has spread throughout the community (Winata et al. 1995). Genetic research indicates that the mutation that causes deafness first occurred between approximately four and nine generations ago (Winata et al. 1995). Notwithstanding the biological time depth of this mutation, the first substantial cohort of deaf signers did not appear until five generations ago, and this event marks the emergence of Kata Kolok (de Vos 2012). Furthermore, similar to Kisch' description of the Al-Sayyed Bedouin community (see Kisch, this volume), deaf adults in Bengkala are not always easily assigned to a single generation because their parents may have been born into different generations, and their peers belong to different generations as well (de Vos 2012). The grammatical description of perfective aspect in Kata Kolok, which is presented in section 4, is primarily based on corpus analyses of deaf signers of the fourth biological generation of signers, who are currently between twenty and sixty years old. The acquisition data stem from a child from the youngest generation of Kata Kolok signers.

In the year 2000, the village of Bengkala was home to 2,186 individuals, of whom 47 were deaf (Marsaja 2008). Based on a linguistic survey conducted in that same year, we learnt that as many as two-thirds of the hearing community members use Kata Kolok, albeit with varying degrees of proficiency (Marsaja 2008). A demographic survey conducted in 2008 has indicated that the village population has increased to 2,740 (Astika 2008). Assuming that the proportion of hearing signers has remained constant, Kata Kolok could be currently used by up to 1,800 hearing signers. Furthermore, a fieldwork visit by the author in September 2011 has indicated that only 38 out 46 deaf individuals born into the community are permanently based within the community (see also the socio-linguistic sketch of Kata Kolok in this Volume). The overall ratio of deaf to hearing signers in Bengkala could thus be estimated as 1:47.

Due to the high proportion of signers in the village, deaf children grow up in a linguistic setting quite similar to hearing children, in terms of acquiring language from birth. Usually their parents can sign, in addition to most of their neighbours and the children they play with. Furthermore, ethno- 
graphic observations of Kata Kolok have revealed the existence of a special register for child-directed signing (Marsaja 2008; see also Nonaka 2004 on child-directed signing in Ban Khor Sign Language). There are currently no existing studies of first language acquisition in such a uniquely rich signing environment, and for this reason, documentation activities by the author have systematically included child signing data (see section 3). Because this language acquisition setting is optimally similar to the native acquisition of spoken languages, differential developmental stages are more easily attributed to differences in the language modality, that is to say to the physical constraints of the organs involved in language production and perception.

In recent years, multiple deaf teenagers from Bengkala have entered the deaf boarding schools in Bali. These adolescents have become fully bilingual in Indonesian Sign Language and Kata Kolok, and such contact situations often result in linguistic change in favour of the majority language associated with perceived educational and professional opportunities. Attendance at this deaf boarding school has also resulted in increased contact between the Kata Kolok community and the larger deaf community of Bali, resulting in changing marital patterns. That is, the intensification of contact between the Kata Kolok signers and Indonesian Sign Language users has also resulted in an increasing number of deaf individuals from Bengkala seeking out deaf spouses from surrounding villages and other parts of Bali. Because deaf individuals outside of Bengkala are not carriers of the identical recessive gene causing deafness, these couples are unlikely to bear deaf offspring (de Vos 2012).

Moreover, this latter tendency, to marry outside the village, is also observed in hearing villagers from Bengkala due to recent socio-economic changes. That is to say, an increasing number of hearing community members has found employment in the tourist industry in the South of Bali, and they end up marrying individuals from other parts of the island. In effect, these changing marital patterns dilute the prevalence of the recessive gene in the population of Bengkala and the incidence of deafness as a result. Even though Kata Kolok is still used by hundreds of hearing signers, chances are that the communicative need for the sign language will rapidly disappear when the number of deaf individuals decreases significantly. Since 2005, no deaf children have been born to parents using Kata Kolok, and this makes the study of its acquisition especially pressing, as another opportunity to study the acquisition of this endangered sign language without the influence of Indonesian Sign Language may not readily occur (de Vos 2012).

Interestingly, in response to this imminent threat, the DeafAlliance-a team of deaf and hearing villagers who advocate the interests of the deaf villagers 


\section{Connie de Vos}

and their relatives - have supported the establishment of Kata Kolok-based deaf education. This inclusive education programme takes the form of a deaf unit within one of the village's elementary schools and is currently attended by eight deaf children (including those described below). In general classes such as religion and gymnastics, the deaf children join the hearing classrooms, but in math and literacy they are exclusively taught within the deaf unit. This deaf unit has been supported by regional and national governments since 2007. Initial observations indicate that Kata Kolok's lexicon is rapidly expanding, presumably because of increased contact with Indonesian, and the youngest deaf children are among the first generation to receive speech therapy. The initiation of deaf education may thus prove to have a profound impact on Kata Kolok's lexicon, and perhaps prompt the emergence of other contact-induced features, such as mouthings (cf. de Vos 2011). At any rate, the sign language use of this youngest generation of signers embodies the locus of linguistic change of this remarkable sign language.

\section{Corpus analysis}

\subsection{Linguistic fieldwork \& corpus construction}

Over the past five years, the author has spent 12 months in Bengkala, during which she interacted with deaf signers on a daily basis. ${ }^{3}$ She has participated in Hindu ceremonies and deaf gatherings, and initiated the establishment of the deaf unit within the village's elementary school (Kortschak 2010; de Vos 2012; de Vos \& Palfreyman forthcoming). This type of participatory linguistic fieldwork allowed her to familiarise herself with local customs, and acquire sufficient fluency in the language. During this time she also coordinated the creation of a digital archive of the language: 100 hours of video data capturing the main contexts in which the language is used. The Kata Kolok corpus currently includes spontaneous video recordings of all deaf Kata Kolok signers as well as a number of hearing signers. This digital repository covers a wide variety of data: culturally entrenched narratives of deaf ghosts, the Bali bombings, and Balinese cock fights in multiple participant configurations; stimulus-based elicited signing; and a special section devoted to child signing.

The documentation of Kata Kolok has resulted from the joint efforts of multiple individuals, not in the least the deaf and hearing community members of Bengkala who agreed to be recorded. Ketut Kanta was born and raised in Bengkala and is a fluent Kata Kolok signer. As a research assis- 
tant he made many video recordings, including longitudinal recordings of multiple deaf children within the village (more detail is provided below). Ketut Kanta has also provided sentence-level translations of a number of the video recordings. English translations of these Indonesian transcripts were provided by Febby Meilissa - a research assistant at the Jakarta Field Station of the Max Planck Institute for Evolutionary Anthropology. It is hoped that these Indonesian and English translations will make the corpus accessible to a national and international (academic) audience in future. The digitisation of all video recordings has been facilitated by Nick Wood and supported by the Max Planck Institute for Psycholinguistics. All data have been deposited by the author and have since been jointly archived by the Max Planck Institute for Psycholinguistics and the International Institute for Sign Languages and Deaf Studies. The video files are also stored locally within Bengkala's village administration.

As mentioned above, a special section of the Kata Kolok corpus targets child signing. From mid-2007 until mid-2009, recordings were made of two deaf children born into deaf families, who were aged 23 and 24 months at the time of the first recording. The parents and older siblings of each child are native Kata Kolok signers. All child signing recordings were made by Ketut Kanta, who has known the deaf families in the village for many years and has worked with them on several occasions. Consequently, he is a familiar face for the children involved in this project, and thus particularly well suited to make the recordings. During the course of the project he has also become the main teacher of the deaf children at the village's school, which was set up in July 2007 in collaboration with local authorities (de Vos \& Palfreyman forthcoming). Recordings were made once or twice a month in systematically varied situations: interacting with a parent or caregiver, interacting with each other or with other deaf and hearing children, and in free play. Each recording session lasted on average half an hour. This resulted in approximately 50 hours of video data of the children between the ages of 23-49 months and 24-48 months.

\subsection{Selected data and transcription}

Adult data. The description of temporal aspect in Kata Kolok in section 4 is based on analyses of the section of the Kata Kolok corpus that features spontaneous signed conversations of exclusively deaf (native) signers. The frequency of the perfective marker was determined on the basis of a subset of those corpus files that had been transcribed in detail. These files comprise 


\section{Connie de Vos}

various participant configurations, including five monologues, seven twoparticipant conversations between signers who have intimate knowledge of each others' lives, and one group conversation. This data set totals six and a half hours of densely transcribed video data, with thirteen different signers represented. The corpus analyses have also been supplemented by ethnographic observations that were made by the author during the extensive periods of fieldwork.

Child data. Due to space considerations, the present study focuses on montly video recordings of one child (henceforth Child 1), between the age of 24 and 36 months. A total of 5.5 hours were selected: 164 minutes between 24-30 months of age and 164 minutes between 32 and 36 months. Due to technical difficulties at the field site no data is available at 26 and 31 months of age.

The initial parts of the video file names presented throughout the paper refer to the entire video file as it was added to the Kata Kolok corpus. Based on this, the reader can thus consult metadata on that file by viewing the corpus online at the following URL: http://corpus1.mpi.nl, and by subsequently navigating to the relevant section by opening the following corpus branches: Sign Language, Sign Language Typology, Village Sign Languages, Bali, Vos, Kata Kolok, Child Signing, Longitudinal, Deaf Children, Child 1.

Both the adult and child signing data presented in this paper are based on corpus transcriptions that were made by the author. These transcription activities were greatly facilitated by the Indonesian and English translations provided by Ketut Kanta and Febby Meilissa as well as the author's own knowledge of the language. The data have been annotated and coded using ELAN annotation software, which is freely available at http:/www.lat-mpi. eu/tools/elan. ELAN enables the researcher to make time-aligned video annotations on multiple tiers, which can be created and arranged according to the nature of the research questions. The coding scheme that has been used throughout the Kata Kolok corpus is based on the transcription format developed by the Sign Language Typology Group at the Max Planck Institute for Psycholinguistics in Nijmegen in June 2005. ${ }^{4}$

\section{Perfective and imperfective aspect in Kata Kolok}

Linguistic tense refers to the timing of an event in terms of future, present or past. The English -ed marker, for instance, transforms a verb into a past tense form. In Kata Kolok tense is not marked on verbs (de Vos 2012). 
From a cross-linguistic perspective this is not surprising; there are not many reports on the marking of tense on verbs in sign language literature (but see Jacobowitz \& Stokoe 1988; Schermer \& Koolhof 1990; Sapountzaki 2007), and additionally, many spoken languages lack past and future tense marking (Dahl \& Velupillai 2011a-b). In contrast to grammatical tense, which marks a past/future distinction, perfective/imperfective aspect is primarily concerned with whether or not an event is completed (for a short introduction to the distinction between tense and aspect see Dahl \& Velupilai 2011d). In a sample of 222 spoken languages, aspect is marked grammatically in nearly half of the cases, and aspectual systems are not uncommon to sign languages either (Dahl \& Velupillai 2011c; Sandler 1990; Zeshan 2003).

In South-East Asian Languages, perfective aspect markers are frequently derived from content words that mean 'finished, already', and this is also the case in Indonesian (sudah) and Balinese (telah) - the spoken languages that are in cross-modal contact with Kata Kolok (Dahl \& Velupilai 2011c). In Kata Kolok, too, temporal aspect revolves around the perfective/imperfective distinction. The perfective marker FINISH\#pah is elsewhere identified as a completive aspect and is expressed by a sign that also means 'finished, already' (de Vos 2012). Marsaja (2008:201) previously described the sign KONDEN 'not-yet' in Kata Kolok as a negative completive. This manual form is glossed as NOT-YET in this paper, and it is formed by a B-hand making a downward movement. The present paper adopts the terms perfective and imperfective respectively, in line with the literature on spoken Balinese and Indonesian and the literature on the acquisition of aspect. The analyses presented in this paper will focus on the marking of perfective aspect as corroborated by the corpus analyses described below and verified by intuitions of various native and fluent Kata Kolok signers.

In its full form, the sign FINISH is produced with two 5 hands rapidly turning palm upward along with a lip smack glossed as 'pah'. In phonetic terms this lip smack is a bilabial glottalised ingressive. Figure 1 below illustrates the initial and the final position of the full form. Neither the manual perfective marker nor its non-manual counterpart 'pah' have been observed in Balinese co-speech gesture, and they do not have any transparent nonlinguistic communicative function. When acquiring the appropriate uses of the perfective marker the child thus relies on the syntactic distribution that is specific to Kata Kolok, and cannot rely on gestural uses of these forms. As becomes clear from the figure, the final mouth position of the perfective marker vaguely resembles the mouth aperture in the pronunciation of sudah and telah and might therefore be considered a mouthing - a meaningful 


\section{Connie de Vos}

mouth movement derived from a spoken word. In the perception of a number of hearing Kata Kolok signers, however, the overlap between these forms seems incidental. Moreover, the lip smack in itself has telic characteristics in terms of its abrupt production. It is presumably this temporal iconicity that has motivated the use of a very similar form as an adverb meaning 'finally!' in American Sign Language as well (Anderson \& Reilly 1998). Unlike Kata Kolok, however, the American Sign Language form PAH! has not been analyzed as a perfective marker, and PAH! is not linked to the American Sign Language form of FINISH in any way.

In adult Kata Kolok signing, the perfective marker is most frequently produced with one hand, but is accompanied by the non-manual component without exception. As will become clear in section 5, the coordination of the manual and non-manual components of this grammatical marker constitute a challenge to the child under consideration here.
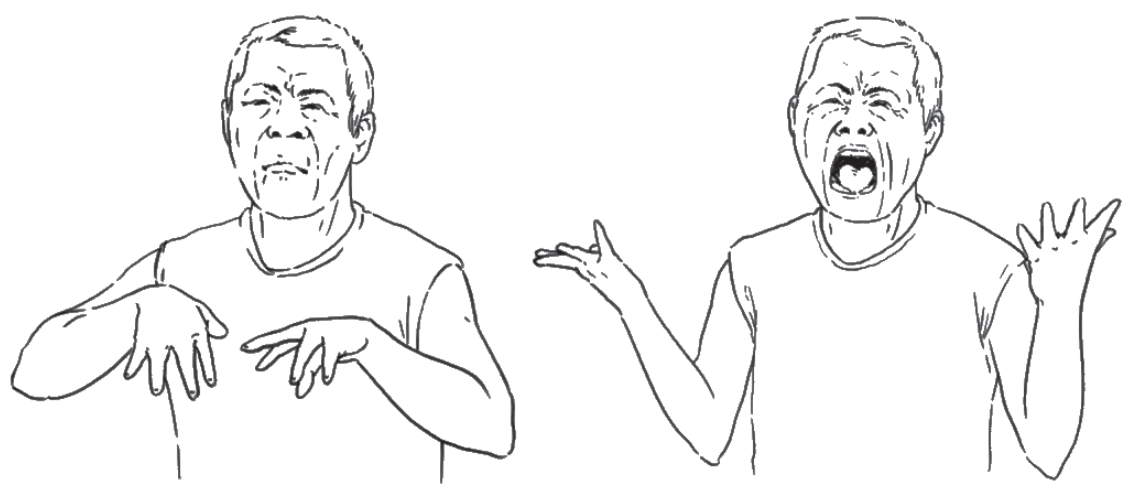

Figure 1. The initial and the final frame of the full perfective aspect marker

The perfective marker forms a crucial component of daily greetings among the inhabitants of Bengkala. In the early evening, the villagers take their mandi 'bath' and have dinner before visiting their relatives and friends for a chat. Upon arrival, and rather than asking how one is doing, a polite way of greeting someone is to ask whether they have had their baths yet, and whether they have had their dinners yet. ${ }^{5}$ Both questions are formed respectively by producing the signs EAT and BATH alongside raised eyebrows and a nod. The designated response is a one-handed FINISH\#pah. In addition to the prominent use of FINISH\#pah in these greetings, the sign is also extremely frequent in spontaneous language use. The analysis of six and half hours of spontaneous Kata Kolok discourse has identified 272 instances of FINISH\#pah in a total of 10,106 manual signs. With an incidence of 2.7\%, 
FINISH\#pah is the third/fourth most frequent sign in the corpus alongside the general negation marker NEG. The sections below present further types of discourse contexts in which the perfective marker is used.

An instance of use of the full form is illustrated below by Example 1. Before explaining this signed example in detail, the transcription conventions adopted in this paper are briefly addressed. The glosses throughout this paper are presented on three independent rows: firstly, NM (Non-Manual) indicates non-manual signals such as facial expressions and body movements; secondly, MG (Main Gloss) is used for signs produced by the dominant hand, or signs that are two-handed; and thirdly, signs produced with the non-dominant hand are presented on the bottom row ND (Non-Dominant hand). In line with conventions used in the field of sign language linguistics, glosses for lexical signs are presented in capital letters. The transcription on multiple independent rows allows for the visual representation of simultaneous signals in the signed sentences. The initial pointing sign in Example 1 , for instance, is produced with raised eyebrows ( $\mathrm{rb}$ ). When simultaneous signals are described in the text the \# symbol is used. The simultaneous uses of the non-manual and manual parts of the perfective aspect marker are thus indicated as FINISH\#pah.

Returning to this fully-fledged form, Example 1 presents an extract of a story about a motorbike accident in which two deaf men died. The signer, the father of one of these men, has just described how his son died directly following a collision with the truck. The other man, a Muslim friend of his son, did not die until a few days later in the hospital. The signer explains that after the accident the injured man was taken to the hospital in the nearest city of Singaraja and was given intravenous fluid. Despite the doctors' best efforts, the man passed away in the hospital after five days.

\section{Example 1}

$\mathrm{NM} \quad \mathrm{rb}$

MG IX'Singaraja’ STICK-NEEDLE INFUSION++ FINISH ND

'There (at the Singaraja hospital), he was given intravenous fluid.'

NM

MG

ND

'After five days, (the deaf Muslim) also died. $\mathrm{pt}$
DIE
COUNTING'one, two, three, four, five'
DIE

FINISH_Gta6oct7.mpg 


\section{Connie de Vos}

When the non-manual perfective marker is attached to a lexical predicate, it can occur without the manual part. Conversely, the manual part of the perfective does not occur without the non-manual component in the spontaneous corpus of adult Kata Kolok signing. In Example 2 the signer combines the sign GO-FROM-HERE-TO-X with the non-manual component of the perfective marker, which has been glossed as 'pah'. The example comes from a narrative about an encounter with a deaf ghost (see also the socio-linguistic sketch of Kata Kolok in this Volume). Although the ghost was deaf, it did not want to talk to the narrator and disappeared in the direction of location ' $\mathrm{X}$ '.

Example 2 Non-manual perfective with predicate I

NM pah

pah

MG GO-FROM-HERE-TO-X GHOST TALK GO-FROM-HERE-TO-X ND

'The ghost went away, (I tried to) talk, (but it) went.'

Gta60ct7_GO-AWAY\#perfectiveaspect.mpg

Another sentence in which the non-manual perfective marker is used with a predicate is shown in Example 3 below. This utterance comes from a narrative by a signer who discusses a financial dispute between his son and daughterin-law, who live in the same compound. The signer explains that he had already given them three hundred thousand rupiah (RED ${ }^{\wedge}$ THREE). (100,000 Indonesian rupiah bank notes are red and in Kata Kolok are referred to as such.) Importantly, although the translation indicates that the event happened in the past by using past tense, the Kata Kolok sentence only indicates that the event is completed, whether in the future or in the past. It is only through pragmatic implication that the historical interpretation arises. De Vos (2012) addresses the relationship between perfective marker and temporal inference in more detail.

\section{Example 3 Non-manual perfective with predicate II}

NM pah

MG GIVE ME RED`THREE

ND

'I had given/gave (them) three hundred thousand rupiah.'

Gta60ct7_GIVE\#perfectiveaspect.mpg

As shown above, FINISH\#pah is extremely frequent in the Kata Kolok corpus, but how about the non-manual marking of predicates with 'pah'? Facial expression has not been systematically transcribed in the present Kata Kolok corpus. In order to assess the frequency of the bound morpheme 'pah', five one-minute stretches of densely transcribed video data were therefore 
sampled from two dialogic conversations and one monologue narrative by five different signers. In these selected stretches of spontaneous discourse, 546 manual signs occurred, including 6 instances of FINISH\#pah. The non-manual aspect marker 'pah' occurred 7 times with a predicate. Three instances concerned the sign THROW\#pah, two of which followed each other closely in a monologue regarding Indonesia's colonial period, and the third instance was used by a deaf woman whilst gossiping about a quarrel between her neighbours. GO-AWAY\#pah occurred four times and was used by two signers, discussing the prices of rice in the different markets of the surrounding villages and the whereabouts of the deaf teenagers. In this stretch of discourse the perfective marker FINISH is thus slightly less frequent than in the overall corpus, but appears equally frequently as the non-manual perfective marker. It is unclear at present what motivates the use of one form over the other, and further analyses are required to determine this. In any case, it would appear that if children acquiring Kata Kolok have a preference for one form over the other, this is not easily explained by an uneven distribution of forms in adult Kata Kolok signing. Recall from the discussion above that child-directed signing may sometimes disfavor the use of non-manual markers because of the associated affective meanings in American Sign Language (Reilly \& McIntire 1991). The non-manual marker 'pah' does not have such a negative connotation however and for this reason it is unlikely that the form would have a different distribution in child-directed Kata Kolok.

Finally, the non-manual part of the perfective marker can also be used with predicative pointing signs. Example 4 presents an example of such a combined structure produced by an 8-year-old deaf girl with deaf parents, who is telling a story about a ghost she saw the night before. She discusses how her neighbour, who is said to have supernatural powers, turns into a ghost at night. This ghost went down a path near to the signer's current setting. She uses her non-dominant hand to indicate that path, tracing it along the horizon with her index finger. The pointing sign ends in the use of the non-manual aspectual marker glossed as 'pah' and produced by smacking the lips. While she holds that sign, she produces the sign GHOST with her dominant hand. She then indicates the location where the ghost stopped again by pointing at that location and producing the aspectual marker along with it. The fact that this pointing sign is produced with the aspectual marker indicates that it is treated as a predicate parallel to lexical signs that can be marked in a similar way. The marking of pointing signs by 'pah' and other grammatical nonmanuals are taken as evidence for the syntactic integration of pointing signs in Kata Kolok (de Vos 2012). 


\section{Example 4 Non-manual perfective marker combined with pointing signs NM pah \\ MG GHOST \\ ND IX'tracing path' pah \\ '(It) went along that path, the ghost, and then it stopped.'}

CGSb14aug7_IX_trace_perfective_aspect.mpg

The sections above have shown that the perfective marker in Kata Kolok occurs both as a fully-fledged form (FINISH\#pah), and as a non-manual form ('pah') that attaches to lexical predicates and pointing signs. FINISH\#pah is highly salient in the language, as evidenced by its frequency in a corpus of spontaneous Kata Kolok signing and its use in ritual greetings. The occurrence of the non-manual perfective marker alongside predicates had not been systematically transcribed within the corpus but a randomly selected five minutes indicates that this use of 'pah' might be equally frequent as FINISH\#pah. In contrast to FINISH\#pah, the use of the non-manual perfective marker alongside pointing signs is relatively infrequent: out of 1,183 index finger pointing signs, only a handful of cases has been identified (de Vos 2012). The non-manual form 'pah' does not occur in isolation in adult Kata Kolok signing and for this reason it is best analysed as a bound morpheme.

\section{An exploration of the perfective in Kata Kolok child signing}

In light of previous work on the acquisition of non-manual morphology, the following questions arise with regard to the acquisition of the perfective marker in Kata Kolok. First of all, by what age does the child acquiring Kata Kolok start to produce both the non-manual and manual perfective markers? Secondly, by what age does the child data start to show the adult distribution of perfective forms? And finally, is there evidence that the manual perfective form takes precedence over the combined non-manual and manual forms in Kata Kolok acquisition, as suggested by Reilly (2006) for American Sign Language?

In order to address these issues, the author analyzed monthly video recordings of a deaf preschooler from 24-36 months of age who is acquiring Kata Kolok natively. As was argued in section 2, the setting in which deaf children acquire sign language in a deaf village is optimally comparable to the acquisition of a spoken language in terms of exposure to the language from birth, numbers of communicative partners and the variety of settings 
in which the language is used. These transcription activities identified 1,119 manual signs, of which $458(41 \%)$ were pointing signs. The total data set counts 84 instances of the perfective marker, including the grammatical form FINISH\#pah, and the grammatical co-production of 'pah' with pointing signs (IX\#pah) and predicates (PREDICATE\#pah), as well as ill-formed instances of 'pah' and FINISH in isolation. ${ }^{6}$ Notably, while the phonological development of these forms could be of interest as well, this paper is mainly concerned with the syntactic distribution and coordination of non-manual and manual perfective forms. There currently are no deaf children within the relevant age range (4-5 years) to test the comprehension of perfective forms by Kata Kolok child signers. The analyses below therefore focus on the spontaneous production of perfective forms in child signing as it differs from the adult use in spontaneous Kata Kolok discourse (see section 4). ${ }^{7}$

\subsection{The full form of the perfective marker - FINISH\#pah}

The sign FINISH was produced 32 times (seven times with two hands). Table 1 presents an overview of the grammatical and ungrammatical instances of this manual perfective marker. FINISH is produced for the first time at 28 months, but without the compulsory non-manual marker 'pah'. The lexical sign FINISH\#pah is produced alongside the non-manual marker 'pah' for the first time at 29 months. By 34 months the sign has a considerable frequency (11 times out of 138 manual signs $(8 \%)$ ). The fact that the child still produces ungrammatical forms, lacking the obligatory non-manual marker, at 34 and 35 months, suggests that he may not have fully acquired the perfective marker, however.

Table 1. The use of the manual perfective marker by a Kata Kolok signer between 24-36 months of age.

\begin{tabular}{lccc}
\hline & $\begin{array}{l}\text { Grammatical forms: } \\
\text { FINISH\#pah }\end{array}$ & $\begin{array}{l}\text { Ungrammatical } \\
\text { forms: FINISH }\end{array}$ & $\begin{array}{l}\text { Total instances of the } \\
\text { manual perfective } \\
\text { marker }\end{array}$ \\
\hline 24 months & 0 & 0 & 0 \\
25 months & 0 & 0 & 0 \\
26 months & No data available & & \\
27 months & 0 & 0 & 0 \\
28 months & 0 & 1 & 1
\end{tabular}




\begin{tabular}{lccc}
29 months & 2 & 0 & 2 \\
30 months & 2 & 0 & 2 \\
31 months & No date available & & \\
32 months & 0 & 0 & 0 \\
33 months & 2 & 2 & 4 \\
34 months & 10 & 1 & 11 \\
35 months & 6 & 4 & 10 \\
36 months & 2 & 0 & 2 \\
& & & total \\
\hline
\end{tabular}

Example 5 below illustrates one of the first instances of the full form of the perfective marker (FINISH\#pah). In many of the sessions, there is at least some reference to the camera being used for recording. In the example below, the child signs FINISH\#pah, followed by a pointing sign at the camera. Although his utterance would suggest otherwise, this sentence occurred in the middle of the session. While the child uses the form FINISH\#pah in a syntactically appropriate slot, he has not fully grasped the aspectual meaning of the perfective marker.

\section{Example 5 FINISH\#pah at 29 months \\ NM Pah pah \\ MG FINISH IX'camera' FINISH IX'camera' \\ ND}

'It (the camera) is finished.'

FINISH\#pah_CSB10oct7.mpg

As explained earlier, most deaf children in Bengkala grow up surrounded by many fluent signers, including hearing adults who live in the same compound or nearby. Example 6 (produced at 34 months) was recorded during a casual conversation of Child 1 with one of his hearing neighbours, a semi-fluent Kata Kolok signer. The child had just been given a snack: some rice and meat. The neighbour signed MEAT\#rb\#nod, thus asking what happened to the meat. The child answered his question by indicating that he had already eaten the meat. This use of the form FINISH\#pah is both contextually and syntactically appropriate. 
Example 6 FINISH\#pah at 34 months

NM

pah

MG

MEAT FINISH

ND

'The meat is finished.'

\section{FINISH\#pah_CSB9mar8.mpg}

As described above, FINISH\#pah is an integral part of daily greetings, and as in Example 6, children are frequently prompted to produce this form in response to similar questions. Of all the instances of FINISH\#pah and *FINISH in Table 1 there is only one example that goes slightly beyond this ritualised response. Example 7 illustrates how the child initiates a conversation with Ketut Kanta, by declaring that he has eaten and his stomach is full.

\section{Example 7 Creative construction at 35 months NM \\ MG EAT FULL-STOMACH FINISH \\ ND \\ 'I ate and my belly is full.'}

The manual form FINISH has an overall incidence of $2.9 \%$ within the child corpus, which is close to the adult use of this form (2.7\%). However, all but one of these forms (see Example 5 above) is produced in response to the questions 'Have you eaten?' and 'Have you bathed?' While these forms are culturally salient in Bengkala, they are infrequent in the adult corpus of spontaneous Kata Kolok signing and it would appear that the frequency of these question types in child-directed signing is a factor in this matter. The child-directed utterances within the Kata Kolok corpus should be coded and compared to regular adult signing to determine such an influence.

\subsection{The non-manual perfective marker - 'pah'}

As was described in section 2, the non-manual part of the perfective marker may also occur along with both lexical predicates and pointing signs, but is ungrammatical on its own. The transcription protocol allowed the nonmanual marker 'pah' to be coded independently of the manual form. Table 2 presents an overview of the instances of 'pah' as it is produced simultaneously with pointing signs, lexical predicates, and also its ungrammatical use in isolation. The table reveals that the child starts to produce 'pah' at 25 months, that is, three months before the manual perfective marker occurs. Based on naturalistic observation of two bilingual Indonesian-Italian chil- 


\section{Connie de Vos}

dren, Soriente (2007) reports the first instances of sudah - the Indonesian perfect particle - by 19 months. In the case of Kata Kolok, 25 months may also not be the actual earliest use of the form by this child, but this study is naturally limited by the recorded data. Tomasello \& Stahl (2004) raise the issue of what statistical conclusions we can draw from developmental corpora, and provide an interesting account of how this can be done by taking the incidence of the linguistic structures into consideration. The perfective marker is among the most frequent structures in Kata Kolok, which increases the reliability of the findings reported below.

The coordination of 'pah' with manual forms appears right from the start, but only alongside lexical predicates and pointing signs. In the adult corpus, instances of IX\#pah are extremely rare: less than $0.05 \%$ of index finger pointing signs are marked by the non-manual perfective marker. Similarly, 14 out of 458 annotated pointing signs are marked in this way, amounting to $0.03 \%$ of the pointing signs in the child data. As was described in section 4, the integrated forms of PREDICATE\#pah are possibly as frequent as FINISH\#pah in adult Kata Kolok signing (up to $2.7 \%$ ). In the child data this combined form is attested in only 17 instances of all 1,119 manual signs $(0.02 \%)$. The combination of the non-manual marker with predicates and pointing signs suggests that there is nothing inherently difficult about the co-production of manual and non-manual components, and that the coordination of manual and non-manual forms of the same grammatical structures is, as Reilly \& Anderson (2002) suggest, a unique challenge in learning a signed language.

Table 2 also reveals that the child produces 'pah' in isolation, without the co-production of any manual forms. The non-adult forms were contextually embedded in each case. Most cases concerned responses to the question 'Have you eaten?' In two cases the child repeated the non-manual aspect marker after it occurred alongside a predicate. From 33 months onwards, the child stops producing the ungrammatical, isolated forms of 'pah', suggesting that he has acquired this specific formal rule concerning perfective aspect in Kata Kolok.

Table 2. The use of the non-manual perfective marker by a Kata Kolok signer between 24-36 months

\begin{tabular}{ccccc}
\hline & $\begin{array}{c}\text { Grammatical forms: } \\
\text { IX\#pah }\end{array}$ & $\begin{array}{l}\text { Ungrammatical } \\
\text { forms: FINISH }\end{array}$ & $\begin{array}{l}\text { Total instances } \\
\text { of the manual } \\
\text { perfective marker }\end{array}$ \\
\hline 24 months & 0 & 0 & 0 & 0 \\
25 months & 1 & 5 & 6 & 12
\end{tabular}




\begin{tabular}{llclr}
26 months & \multicolumn{3}{c}{ No data available } & \\
27 months & 0 & 0 & 0 & 0 \\
28 months & 0 & 0 & 0 & 0 \\
29 months & 2 & 0 & 1 & 3 \\
30 months & 0 & 0 & 0 & 0 \\
31 months & \multicolumn{2}{c}{ No data available } & & \\
32 months & 0 & 10 & 9 & 19 \\
33 months & 7 & 7 & 0 & 14 \\
34 months & 1 & 0 & 0 & 1 \\
35 months & 0 & 0 & 0 & 0 \\
36 months & 3 & 0 & 0 & total
\end{tabular}

Example 8 illustrates one of the first instances of the non-manual perfective marker. Many of the deaf families in Bengkala own livestock such as chickens, pigs, and one or two cows. From a young age, boys in particular are encouraged to contribute to the household by gathering grass for these animals. During the recording session in which this non-manual perfective marker was produced, the child was playing on his parents' farm with an arit (a sickle) and keranjang (a basket woven from bamboo leaves). In the video, the research assistant and the boy were sitting casually in the ground. Without prompting, the boy explained that the knife he is using is broken.

\section{Example 8 Non-manual perfective marker at 25 months \\ NM pah pah \\ MG BREAK BREAK \\ ND \\ 'It (the knife) is broken.'}

BREAK\#pah_CSB8june7.mpg

Example 9 stems from the same causal conversation described in example 5 above. In this case, the child's hearing interlocutor asks the boy where his older sister is by (incorrectly) producing her sign-name. The boy repeats his sister's sign-name, correcting the sign's orientation. Subsequently, he produces a rapid brow raise, which functions as a question tag in Kata Kolok (Marsaja 2008:202-211). Then, the boy indicates that his sister has already gone away (to a deaf boarding school in Jimbaran, in the South of Bali). 
The predicate GO-AWAY is produced simultaneously with the non-manual perfective marker.

Example 9 Non-manual perfective marker at 34 months

NM

$\mathrm{rb}$ pah pah

MG SIGN-NAME'D' GO-AWAY GO-AWAY

ND

'What about D.? - She went away, she went away.'

GO-AWAY\#pah_CSB9mar8.mpg

\subsection{Aspect and telicity}

The predicates that attracted the non-manual and manual perfective marker are limited: BREAK, EAT, THROW, GO-AWAY, FALL, and FLASH. This observation thus corroborates the cross-linguistically robust finding that children initially use the perfective marker with telic predicates such as 'break' and 'throw' while the onset of the less frequent combinations of a perfectiveatelic and imperfective-telic are delayed. Such combinations are therefore taken as a first indication that the child is starting to deduce the grammatical meaning of these aspectual markers. The first instance spotted in this corpus is presented in Example 10 below. At age 33 months the child uses the nonmanual perfective marker with an atelic predicate ('sleep'). As becomes clear from Example 10 however, he combines the non-manual perfective marker with the manual form of the imperfective particle NOT-YET.

\section{Example $10 *$ NOT-YET\#pah at 33 months}

NM pah *pah

MG SLEEP IX SLEEP NOT-YET

ND

'I slept there.'

*NOT-YET\#pah_CSBb19feb8.mpg

The form NOT-YET\#pah in Example 10 represents an intriguing error that results from the simultaneous nature of perfective marking in the language. Given its use with the atelic predicate SLEEP within a sentence it may indicate that the child is on the verge of a developmental stage, starting to grasp the true meaning of FINISH\#pah. In support of that interpretation, the child stops producing ungrammatical, isolated forms of 'pah' at the same age (at 
33 months, see Table 2). From 33 months onwards, the child also tentatively started to use fewer forms of 'pah' with pointing signs, thus moving toward an adult distribution of these forms.

\subsection{Modality-dependent patterns in language development}

The fact that perfective aspect is not fully acquired in the age range examined here makes it impossible to truly test Reilly's (2006) generalisation that the coordinated use of non-manual forms is not acquired before the acquisition of their manual counterparts. However, the fact that the non-manual perfective occurs earlier and more frequently in the corpus, despite the fact that both forms appear equally frequently in adult Kata Kolok, does not counter this interpretation. As such, these findings from Kata Kolok motivate the hypothesis that, with language-specific implementations, children acquiring sign languages may follow modality-dependent developmental stages that can be identified across typologically distinct sign languages. In doing so, they may produce modality-specific errors that result from a difficulty in coordinating manual and non-manual components.

This hypothesis adds to Petitto's (1987) observation that, with modalityspecific implementations, children acquiring American Sign Language follow the same developmental stages (including similar substitution errors) as do hearing children acquiring English. Specifically, her seminal paper showed that deaf children acquire the syntactic distinction between locative and pronominal pointing signs at 25-27 months, around the same time as hearing children acquiring English and Italian learn to use pronouns. As mentioned in section 2, the use of grammatical non-manual markers with pointing signs is taken as key to understanding the syntactic integration of pointing signs within Kata Kolok (de Vos 2012). Taking Petitto's study as a vantage point, then, it is interesting to highlight that the child data in this study indicate that the non-manual perfective marker appears with pointing signs from the start, that is to say, at 25 months. Further exploration of the child signing corpus has identified additional grammatical markers, such as the use of raised eyebrows to indicate a question, and the use of clenched teeth, a non-manual signal which is currently under investigation. These tentative observations suggest that, with language-specific implementations, the acquisition of pointing signs may also exhibit parallels across sign languages. 


\section{Cross-linguistic and cross-modal comparisons in acquisition research}

The linguistic description of village sign languages has contributed considerably to our understanding of the cross-linguistic variability among sign languages (Zeshan \& de Vos, this Volume). Despite its limitations, this paper has aimed to show that recognising this typological diversity is not just a matter of butterfly collecting. By capitalising on these cross-linguistic differences we are able to conduct comparative studies of the acquisition of typologically distinct sign languages for the first time. While temporal aspect is marked in many signed languages, this study presents the first exploration of its development in children. The acquisition of sign language within the context of a deaf village is optimally similar to the situations in which children acquire spoken languages, and comparing the linguistic development of child speakers and signers is thus more likely to reflect genuine differences between the language modalities. Previous research on the acquisition of signed languages has emphasised the stages in language development that are cross-modally robust. The present paper has put forward the hypothesis that there may also be modality-specific developmental steps that occur across sign languages. The simultaneous coordination of non-manual and manual grammatical markers, and more generally, the acquisition of modality-specific structures, inevitably dominates such cross-linguistic investigations. If this approach is extended to include the composite utterances of child speakers, this quest could additionally lead to a deeper understanding of language development from both cross-linguistic and crossmodal perspectives.

\section{Notes}

1. I would like to thank Irit Meir (University of Haifa) and Antonia Soriente (University of Naples 'L'orientale' \& Max Planck Institute for Evolutionary Anthropology) for their comments on a previous draft of this paper.

2. The simultaneous use of manual and non-manual forms is indicated by the \# symbol. Further transcription conventions are addressed in section 4 .

3. I would like to thank the deaf and hearing villagers of Bengkala for welcoming me into their community, and especially Ketut Kanta for his assistance during this period. 
4. Many of the non-manuals that are listed in these transcription conventions are at present unanalysed. The use of non-manual signals for both linguistic and paralinguistic functions is an under-researched area in the description of Kata Kolok.

5. In fact, one of the foreign researchers who worked in the village is now referred to by his 'unusual' greeting: the use of the sign GOOD with raised eyebrows and a smile as in 'How are you?'

6. Please note that italicised capital letters are used here to indicate that PREDICATE is not an actual Kata Kolok sign, but rather represents any lexical predicate within the language.

7. While child-directed signing would have been a better indication of the signed input that this child has received, this aspect of the child-signing sub-corpus had not been transcribed yet.

\section{References}

Anderson, D. \& J. Reilly

1998 PAH! The acquisition of adverbials in ASL. Sign Language \& Linguistics 1. 117-142.

Anderson, D. \& J. Reilly

1997 The puzzle of negation: How children move from communicative to grammatical negation in ASL. Applied Psycholinguistics 18. 4111-429.

Dahl, Ö. \& V. Velupillai

2011a The Past Tense. In: Dryer, M. S. \& M. Haspelmath (eds.) The World Atlas of Language Structures Online. Munich: Max Planck Digital Library, feature 66A. Accessed on 2011-12-17.

Dahl, Ö. \& V. Velupillai

2011b The Future Tense. In: Dryer, Matthew S. \& Haspelmath, Martin (eds.) The World Atlas of Language Structures Online. Munich: Max Planck Digital Library, feature 67A. Accessed on 2011-1217.

Dahl, Ö. \& Velupillai, V.

2011c The Perfect. In: Dryer, M. S. \& M. Haspelmath (eds.) The World Atlas of Language Structures Online. Munich: Max Planck Digital Library, feature 68A. Accessed on 2011-12-17. 
Dahl, Ö. \& V. Velupillai

2011d Tense and Aspect. In: Dryer, Matthew S. \& Haspelmath, Martin (eds.) The World Atlas of Language Structures Online. Munich: Max Planck Digital Library, supplement 7. Accessed on 2011-1217.

De Vos, C.

2011 Kinship in Kata Kolok and Balinese: differences between the signed and spoken language of a single village community. Talk presented at the EuroBABEL workshop on Kinship and Number Systems from Cross-Linguistic and Cross-Modal Perspectives. Preston, 15-16 September.

De Vos, C.

2012 Sign-Spatiality in Kata Kolok: how a village sign language of Bali inscribes its signing space. $\mathrm{PhD}$ dissertation. Nijmegen: Max Planck Institute for Psycholinguistics.

De Vos, C. \& N. Palfreyman.

2012 [Review of the book Deaf Around the World: the impact of Language, edited by Mathur \& Napoli (eds.)]. Journal of Linguistics.

Enfield, N. J.

2009 The Anatomy of Meaning: Speech, Gesture, and Composite Utterances. Cambridge: Cambridge University Press.

Jacobowitz, E. \& W. C. Stokoe

1988 Signs of tense in ASL Verbs. Sign Language Studies 60. 331-340.

Kendon, A.

2004 Gesture: Visible action as utterance. Cambridge: Cambridge University Press.

Kortschak, I.

2010 Where everyone speaks deaf talk. Invisible people: poverty and empowerment in Indonesia (pp. 76-89). Jakarta: PNPM Mandiri.

Li, P. \& M. Bowerman.

1998 The acquisition of lexical and grammatical aspect in Chinese. First Language 18. 311-350.

Marsaja, I. G.

2008 Desa Kolok - A deaf village and its sign language in Bali, Indonesia. Nijmegen: Ishara Press.

McNeill, D.

$2000 \quad$ Language and Gesture. Cambridge: Cambridge University Press. 
Nonaka, N.

2011 Interrogatives in Ban Khor Sign Language: a preliminary description. In G. Mathur \& J.D. Napoli (Eds.) Deaf around the World: the impact of language. London: Oxford University Press.

Petitto, L. A.

1987 On the autonomy of language and gesture: evidence from the acquisition of personal pronouns in American Sign Language. Cognition 27. 1-52.

Reilly, J. \& D. Anderson

2002 FACES: The acquisition on non-manual morphology in ASL. In G. Morgan \& B. Woll (eds.), Directions in Sign Language Acquisition, 159-181. Philadelphia: John Benjamins.

Reilly, J. \& M. L. McIntire

1991 WHERE SHOE: The acquisition of wh-questions in ASL. Papers and Reports in Child Language Development 30104-111.

Reilly, J.

2006 How faces come to serve grammar: The development of nonmanual morphology in American Sign Language (Eds.), B. Schick, M. Marschark, \& P.E. Spencer. Advances in the Sign Language Development of Deaf Children., 262- 290. Cary, NC: Oxford University Press.

Sandler, W.

1990 Temporal Aspects and ASL Phonology. In S. D. Fischer \& P. Siple (eds.), Theoretical Issues in Sign Language Research, vol. 1: Linguistics, 7-35. Chicago: Chicago University Press.

Sapountzaki, G.

2007 Free functional elements of tense, aspect, modality and agreement as possible auxiliaries in Greek Sign Language. Sign Language \& Linguistics 10. 91-99.

Schermer, T. \& C. Koolhof.

1990 The reality of time-lines: Aspects of tense in SLN. In S. Prillwitz \& T. Vollhaber (Eds.) Current trends in Sign Language Research (pp. 295-305). Hamburg: Signum Press.

Soriente, A.

2007 The acquisition of temporal markers in an Italian-Indonesian bilingual child. Talk presented at the Eleventh International Symposium On Malay/Indonesian Linguistics (ISMIL 11). Manokwari, 6-8 August 2007. 
152 Connie de Vos

Tomasello, M. \& D. Stahl

2004 Sampling children's spontaneous speech: how much is enough? Journal of Child Language 31. 101-121.

Van Hout, A.

forthcoming The acquisition of aspect. In J. Lidz, Pater, J. \& W. Snyder (Eds.), Handbook of Developmental Linguistics. London: Oxford University Press.

Zeshan, U.

2003 Indo-Pakistani Sign Language grammar: a typological outline. Sign Language Studies 3(2). 157-212.

Zeshan, U.

2004 Interrogative constructions in signed languages: cross-linguistic perspectives. Language 80(1). 7-39. 


\title{
The survival of Algerian Jewish Sign Language alongside Israeli Sign Language in Israel
}

\author{
Sara Lanesman and Irit Meir
}

\section{Introduction}

We came across Algerian Jewish Sign Language quite accidentally. We were investigating the history of Israeli Sign Language, the dominant sign language in Israel, that emerged in the 1930s, with the formation of the Jewish Deaf community in Israel. One of the tasks we asked our interviewees to do was a simple picture-naming task; we wanted to establish the degree of uniformity in the vocabulary of first and second generation of ISL signers. When we interviewed Y.Z., a 65 year old man who immigrated to Israel from Algeria, he asked us: "Do you want me to use the signs I use with my friends, or the signs I used with my mother?" We were intrigued, and asked him what the difference was. He replied that with his friends he uses ISL, but with his mother he used "Algerian signing". We asked him to sign both. While videotaping him, two things became obvious right away. First, the Algerian signs were very different from the ISL signs. Secondly, it was clear that Y.Z. remembered the Algerian signs very well; these signs were still very much part of his active linguistic repertoire. Every now and then he would comment: "There is no sign for that concept in Algerian signs." Such comments gave further indication that Y.Z. was bilingual in two sign languages, that he kept the two languages apart, and used both.

We started looking for more information on the sign language that he referred to as Algerian sign. Who used it? Where did the language come from? Do people use it until today? Is it passed on to younger generations? As our investigation expanded, we discovered that the language is used among people who came from a specific region in Algeria, the M'zab region, specifically from the city of Ghardaia. These people use the language even today, though almost all of them use ISL as their dominant language. This language, which we termed Algerian Jewish Sign Language (AJSL), contributes to the linguistic mosaic of sign languages in Israel. 
Israel is home to several sign languages. The dominant sign language is Israeli Sign Language (ISL), a language that emerged in the 1930s, with the formation of the Deaf community in Israel and the establishment of the first schools for the deaf. Apparently there were some small signing groups in some towns in the region before that, but little is known about them. The members of the first and second generations of the Deaf community came from different backgrounds, both in terms of their country of origin, and in terms of their language. A few were born in Israel, but the majority were immigrants who came to Israel from Europe (Germany, Austria, France, Hungary, Poland), and later on from North Africa and the Middle East. Some of these immigrants brought with them the sign language of their respective countries (e.g., German Sign Language, Austrian Sign Language, Moroccan Sign Language and others). Others had no signing, or had some kind of a homesign (gestural communication system developed and used among the members of one family, see e.g. Goldin-Meadow 2003). Deaf people started to form a social group that met regularly. In 1944 these social ties were formalised by establishing an association for the deaf in Israel, and creating deaf clubs around the country. Today the community numbers about 10,000 members. The language is quite unified across the country, though there is some regional lexical variation; i,e., some signs are typical of the Tel-Aviv area, while others may be used in Haifa, Be'er-Sheva or Jerusalem. The country of origin of the signers also may have some effect on the lexicon. Some signs are used within families of Moroccan, Algerian, Egyptian or German origin. This latter type of variation is more pervasive among older signers.

In addition to ISL, which developed in an 'urban' setting, there are also several village sign languages that developed in Arab, Bedouin and Druze villages in the country. The most studied of these is Al-Sayyid Bedouin Sign Language (ABSL), a language that emerged about 75 years ago in the Al-Sayyid Bedouin community. The socio-linguistic characteristics of this community were studied and described by Kisch (this volume, 2000, 2007, 2008). Its linguistic structure is described in Sandler et. al (2005), Aronoff et. al (2008) and Padden et. al (2010) and references cited there. Another sign language developed in Kfar Qasem in central Israel. According to preliminary study (Kastner, Meir and Sandler in preparation), deaf children were born into this community in the early 1920, maybe even earlier, so that the sign language that emerged there is probably slightly older than ABSL. Other village sign languages exist in Ein Mahel and Arab El-Naim, a town and a village located in the northern part of Israel. All these sign languages are endangered. People in their 30s and younger attend schools for the deaf 
or deaf classes in regular school in which the teachers use ISL signs. Moreover, recent activities of the Institute for the Advancement of Deaf People in Israel draw together deaf people from the different communities. In such gatherings, people are much more likely to use ISL. Therefore, the signing of children and young adults is heavily influenced by ISL. Yet all the people from the different communities we interviewed, even children, can make a distinction between ISL and the local sign language, and can use the local sign language if they are asked to.

In addition to the languages that emerged and developed in Israel, at least two languages were brought by immigrants and are still used in Israel today: Russian Sign Language (Yoel 2007) brought by immigrants from the former Soviet Union in the 1990s, and Algerian Jewish Sign Language (AJSL), the topic of the present paper, brought by immigrants from the M'zab area in Algeria in the 1960s (see Lanesman and Meir, this volume for a socio-linguistic sketch of the language).

As pointed out above, Algerian immigrants were not the only ones to bring with them a sign language. Immigrants from Germany, Austria, Hungary, Egypt, Morocco and other countries told us that they used a different sign language in their country of origin. ${ }^{1}$ Yet members of the Israeli Deaf community who immigrated to Israel from other countries report that they have forgotten the sign language which they had used in their country of origin. When we asked people to try and remember signs that they used in their country of origin or signs that they used within their families, people often insisted: "I don't remember. I forgot the language. Now I use only ISL." Some people could provide a few signs, but in general, they ceased to use their original sign language long ago. These people exhibit what can be regarded as $L 1$ attrition, that is, the loss of first language by predominant use of the second language. This is very typical of immigrants: "The diminished role of L1 in use and function, exacerbated by separation from the L1 speaking community in the case of immigrants, is one of the significant sociolinguistic variables in the advent and sustenance of first language attrition" (Seliger and Vago, 1991:4). There is not much literature about L1 attrition in sign languages (but see Yoel 2007 for an overview and an analysis of attrition of Russian Sign Language in Russian immigrants in Israel). Yet from our interviews and our acquaintance with the ISL community, it is evident that most members of the community ${ }^{2}$ have forgotten their L1.

This situation stands in a marked contrast with what we found in AJSL users. Although they use ISL daily, even within their nuclear families, they remember their original language and use it to this very day. This special attribute of AJSL users led us to formulate our research question: 
Which factors have contributed to the survival of AJSL in Israel for over two generations alongside ISL? In order to understand the socio-linguistic circumstances that led to the survival of AJSL in Israel, we conducted detailed interviews with nine AJSL users who live in Israel today. In this chapter we describe some of the main results of our research. We first provide a literature review on existing sources about AJSL and the community in which it developed (sections 2 and 3). The research methodology that was used in collecting and analyzing the interviews are detailed in section 4 . The questionnaires were aimed to obtain information about the emergence and use of AJSL in Ghardaia, Alegeria (described in section 5) and the social and linguistic circumstances of its use when the community immigrated to Israel and was confronted with another sign language, ISL (described in section 6). We suggest that the theoretical framework suitable for addressing our research question is that of Ethnoliguistic Vitality (EV, Giles, Bourhis and Taylor 1977), which provides the tools for identifying the factors contributing to the survival of AJSL in Israel in the past two generations, as well as explaining why the language is endangered in the present generation.

\section{Jews in the M'zab}

AJSL developed in several Jewish communities in the region of M'zab in the northern Sahara Desert region of Algeria. This region is isolated from the northern, more densely settled area. The Jewish population in this region lived in several villages and towns, but the main community was in Ghardaia, the largest city of the area.

Ghardaia was founded in the $11^{\text {th }}$ century by Berbers belonging to the Ibadiyya sect, a schismatic Muslim sect who is characterised by a puritanic interpretation of the Koran (Briggs and Guède 1964:9, Nagel 2004:27). The Ibadites, who formed a state around the city of Tahert (often referred to also as Tiaret) in central Algeria, were forced to retreat to the south after their city was destroyed by the Fatimids in $909 \mathrm{CE}$. After more than a century, they settled in the M'zab area, and established a flourishing community there. According to M'zabite and Jewish oral traditions, four Jewish families of craftsmen from Djerba were brought to Ghardaia around the $14^{\text {th }}$ century to work as blacksmiths and jewelers (Briggs and Guède 1964:10). These four families were later joined by Jews from Tamentit, a town situated on the western tip of the north-western Sahara, where a prosperous community developed. In 1447 the community was at the peak of its prosperity, but in 1492, when the Jews were persecuted by the Muslims, they fled and found refuge in the region of M'zab, settling in Ghardaia. 
The Jews living in Ghardaia had several restrictions imposed on them (ibid., p.10). They had to dwell in their own quarter (the mellah) surrounded by a wall, they were not allowed to farm the land, they had to wear black clothes, were not allowed to ride horses or even donkeys, and had to go barefoot outside their own quarter. On the other hand, within their own quarters they were allowed to build a synagogue and perform all the religious and social ceremonies according to their belief and tradition. Marriage was strictly within the community. These conditions gave rise to a closed community. Members of the Jewish community maintained commercial and economical relations with their Muslim neighbours, but other than that, all their social and communal needs were met within their own community. The isolation of this community, however, was not complete. Although the neighbouring towns in the M'zab region did not have Jewish communities, we learned from our interviewees that Ghardaian Jews had some social contact with Jewish people in the towns of Laghouat and Aflou, and that a few Jewish families left Ghardaia and moved to Laghouat and Aflou, probably in search of better sources for livelihood (Joseph Chetrit, p.c. 2012).

Briggs \& Guède (1964) bring some demographic data concerning the Jewish community in Ghardaia, drawn from official archives in the office of the District Commissioner of the M'zab. According to these data, the Jewish community there never exceeded 2,500 people, and usually the numbers were much smaller than that. In 1954, the community numbered 1,091 members. This social isolation, which lasted for at least 500 years, gave rise to a community whose members had several distinct physical characteristics, among them elongated heads, slight tendency towards blond or red hair, and deafness. They also developed some special customs and practices (such as food taboos unrelated to Kosher restrictions. According to these taboos, blind people were prohibited from eating the eyes of a lamb, a lame person was not supposed to eat meat from the leg or foot, and deaf people could not eat tongue, ibid., pp. 33-34), though whether or not these customs are unique to this community or are at least partly shared by other Algerian and North African Jewish communities is a matter of controversy (see e.g. Jacobs 1967).

\section{Deafness in the Jewish community of Ghardaia}

Briggs and Guède's (1964) monograph constitutes a comprehensive description of the lives, customs and traditions of the Jewish community in Ghardaia in the 1950s-1960s, until its last days (in 1962), when the entire community left Algeria and immigrated to Israel and France. In the manuscript there 
are very few mentions of deafness in the community. Yet they constitute the only written source about deafness in the Jewish Ghardaian community, and should therefore be carefully studied, as they provide an initial basis towards our understanding of the life of deaf people in this community.

Briggs and Guède write:

"Inbreeding was doubtless responsible, however, for the relatively high proportion of deaf-mutes among the Jews of Ghardaia, which ran roughly in the neighborhood of 2.5 percent. Luckily for them they were at no great disadvantage in the community, however, for they were treated just like everybody else. Nearly everyone had at least one deaf-mute among his close relatives or neighbor, and so everyone is fluent in sign language. Although these people were completely deaf, they were mute only to the extent that they could not reproduce articulate sounds, words that is. .... Nevertheless, they were extremely noisy. By groaning, grunting, squealing and yelling at the tops of their voices, they called attention to themselves and the ideas which they tried to express by gestures and grimaces. In this way they managed to participate in social activity which they could not enter into as we do by simple conversation. Their efforts in this respect were so successful, however, that they had about as rich a social life as anyone; they had little or no difficulty in finding normal wives or husbands and, in general, seemed very well adjusted. Social gatherings were never so gay and lively as when two or more deaf-mutes were present, for they loved to laugh and delight in acting out their jokes." (ibid., 12).

This short description provides some important pieces of information. The first is the demographic data, namely that deaf people constituted $2.5 \%$ of the population in the community. Second, it seems that deafness ran in several families and was not confined to one family, since many people were in touch with deaf individuals. The observation that "everyone is fluent in sign language" is, of course, extremely important. It asserts that there was a sign language in use in this community, and that its use was widespread enough that even outsiders (such as Briggs and Guède) noticed it. It is not clear what is the basis for their observation that deaf people were fully integrated into the community (that is, whether these were their own impressions or whether they interviewed people concerning this issue), but their description of deaf people in social gatherings clearly show that deaf people took part in the social life of the community, and were able to convey important and intricate information (such as jokes). The fact that deaf people were married, usually to hearing people, is also an important fact indicating that they were socially active in this community.

Since we found no other written sources about deaf people and their language in the Jewish community of Ghardaia, it became clear that in 
order to get more information about the language we would have to use oral histories, that is, to rely on interviews with members of the community. In the following section, we describe our methodology.

\section{Methodology}

We interviewed nine adults, between the ages of 50 and 85 . Seven out of nine participants are deaf, and two are hearing adults who were born to deaf parents. Six of the subjects are women (5 deaf and one hearing) and three men (two deaf and one hearing). Eight were born in Algeria and one was born in Israel. The details of the subjects can be seen in Table 1.

The most important factor in choosing the interviewees was that they are fluent in AJSL and still use it extensively. This is not trivial, since most Algerians in Israel today are bilingual, using ISL to communicate with deaf people not of Algerian origin, and using AJSL only among family members and people from the AJSL community. Therefore it was important to choose interviewees that use the language today with at least several close acquaintances or family members.

We decided to include hearing people in the study, because hearing people are an integral part of the community that uses AJSL, as is evident from the description in Briggs and Guède above, and as we learned from our interviews. In order to gain a deeper understanding of the details and social intricacies of the community and the relationship between deaf and hearing, it is important to interview the hearing members alongside the deaf members of the community.

Three types of data were collected: personal details, life histories and lexical items from the AJSL lexicon. Only the first two are relevant for the present chapter, so we do not expand here on vocabulary elicitation. Personal details were collected by using questionnaires (see Appendix), which included questions about the Jewish community in Ghardaia, the conditions of the deaf in the Jewish community, and the ways of communication between hearing and deaf people in the community. Other questions focused on the immigration to Israel, life in Israel and the use of AJSL in Israel. The questions were presented to the subjects in ISL (by the first author, a fluent ISL signer), and they replied in the same language, as all interviewees are fluent in ISL.

Participants were also asked to share and discuss their life stories with another AJSL signer. These narrations were conducted in AJSL, and their purpose was to enrich our understanding of the socio-linguistic history 
of the community, as well as to videotape AJSL conversations. Both the questionnaires and the conversations were video-taped (with the consent of the participants), and transferred to a digital format. The conversations were then edited using a split screen format. This format enables the viewer to see both subjects in full view and to simultaneously follow both sides of the conversation.

The data was then coded and analysed as follows. Each interviewee's responses to the questionnaire were summarised in writing, creating personal profiles containing personal details with relevance to the research. The life stories were transcribed and translated to Hebrew. They were then divided into short segments, each related to a specific topic. These topics were assembled to a few general themes that directly bear on the research question, namely the survival of AJSL in Israel alongside ISL. The following sections present our findings concerning the language and its community in both locales - Ghardaia and Israel.

Table 1. Personal details of the interviewees

\begin{tabular}{|c|c|c|c|c|c|}
\hline $\begin{array}{l}\text { No. and } \\
\text { Initials } \\
\text { of } \\
\text { subject }\end{array}$ & Gender & $\begin{array}{l}\text { Deaf/ } \\
\text { hearing }\end{array}$ & $\begin{array}{l}\text { Country } \\
\text { of birth }\end{array}$ & $\begin{array}{l}\text { Year } \\
\text { of } \\
\text { birth }\end{array}$ & $\begin{array}{l}\text { Other deaf family } \\
\text { members }\end{array}$ \\
\hline 1. Y.Z. & Male & Deaf & $\begin{array}{l}\text { Ghardaia, } \\
\text { Algeria }\end{array}$ & 1940 & $\begin{array}{l}\text { Two sons, wife, father, } \\
\text { four brothers, more } \\
\text { deaf relatives }\end{array}$ \\
\hline 2. M.G. & Female & Deaf & $\begin{array}{l}\text { Ghardaia, } \\
\text { Algeria }\end{array}$ & 1946 & $\begin{array}{l}\text { Brother, father, more } \\
\text { relatives (sister of } \\
\text { 9.Y.S.) }\end{array}$ \\
\hline 3. M.S. & Female & Deaf & $\begin{array}{l}\text { Aflou, } \\
\text { Algeria }\end{array}$ & 1936 & $\begin{array}{l}\text { Two sisters, brother, } \\
\text { husband, three } \\
\text { children }\end{array}$ \\
\hline 4. L.P. & Female & Deaf & $\begin{array}{l}\text { Ghardaia, } \\
\text { Algeria }\end{array}$ & 1955 & $\begin{array}{l}\text { Three sisters, one } \\
\text { brother, father, more } \\
\text { relatives (sister of } \\
\text { 5.E.S.) }\end{array}$ \\
\hline \multirow[t]{2}{*}{ 5. E.S. } & Female & Deaf & $\begin{array}{l}\text { Ghardaia, } \\
\text { Algeria }\end{array}$ & 1953 & $\begin{array}{l}\text { Three sisters, one } \\
\text { brother, father, more } \\
\text { relatives (sister of } \\
\text { 4.L.P.) }\end{array}$ \\
\hline & & & \multicolumn{3}{|c|}{$\begin{array}{r}\text { Unauthenticated } \\
\text { Download Date | 3/22/19 10:57 AM }\end{array}$} \\
\hline
\end{tabular}




\begin{tabular}{|c|c|c|c|c|c|}
\hline 6. Z.M. & Female & Deaf & Israel & 1957 & $\begin{array}{l}\text { Grandmother, more } \\
\text { relatives (daughter of } \\
\text { 7.M.A.) }\end{array}$ \\
\hline 7. M.A. & Male & Hearing & $\begin{array}{l}\text { Ghardaia, } \\
\text { Algeria }\end{array}$ & 1923 & $\begin{array}{l}\text { Mother, daughter, } \\
\text { two uncles, more } \\
\text { relatives (father of } \\
\text { 6.Z.M.) }\end{array}$ \\
\hline 8. S.S. & Female & Hearing & $\begin{array}{l}\text { Ghardaia, } \\
\text { Algeria }\end{array}$ & 1924 & $\begin{array}{l}\text { Husband, sister, } \\
\text { brother, four children, } \\
\text { more relatives (mother } \\
\text { of 5.E.S. and 4.L.P.) }\end{array}$ \\
\hline 9. Y.S. & Male & Deaf & $\begin{array}{l}\text { Ghardaia, } \\
\text { Algeria }\end{array}$ & 1943 & $\begin{array}{l}\text { Father, sister, more } \\
\text { relatives (brother of } \\
\text { 2.M.G.) }\end{array}$ \\
\hline
\end{tabular}

\section{Deafness and sign language in Ghardaia}

\subsection{The status of deaf people in the community}

Deafness in this community ran in certain families. All our interviewees had other deaf members in their families: E.S. and L.P. (females, age 55 and 53 respectively) have a deaf father and a hearing mother. They also have two other deaf sisters, one deaf brother, and one hearing sister. Y.Z. (male, 65) had a deaf father and a hearing mother. He has three deaf brothers and one hearing sister. M.S. (female, 70) has three deaf siblings and three deaf children, two daughters and one son. M.G. (female, 60) has a deaf father and hearing mother, as well as one deaf brother. Z.M. (female, 50) says: "My paternal grandmother was deaf. Part of my family on both sides is deaf. Part of my family is deaf... In the second and third generations there are many deaf people."

As can be seen, deaf people, both women and men, were married. In Algeria, they were always married to hearing spouses. It is not clear whether there was a restriction on deaf-deaf marriage or that it just never happened. This marriage pattern might indicate that deaf people were considered in need of assistance of a hearing spouse in everyday life. Alternatively, it could also indicate that 'hearing/deafness' status was not an important factor in spouse choice. Our participants did not give a specific reason for this marriage pattern. One participant, M.G., mentioned that what was important 
was getting married; whether the spouse was hearing or deaf was less important. This marriage pattern is different from that of 'urban' signing communities, where deaf-deaf marriage is the norm. In other village communities, the pattern varies. On Martha's vineyard, Groce (1985) reports that deaf people married both deaf and hearing spouses. In Desa Kolok, Marsaja (2008:60) mentions that of the 407 families in the village, there are 13 deaf couples, and two deaf-hearing couples. In the Al-Sayyid community, deaf people used to marry hearing spouses, as in the Ghardaia community, though recently there have been a few deaf-deaf marriages. In Adamarobe, deaf people mostly marry other deaf people, while hearing marry hearing. The Ghana government outlawed marriage between two deaf people, in an attempt to decrease the incidence of deafness. Ironically, most deaf children are born into families with two hearing parents. Deaf women seem to have no trouble getting married, especially in cases of polygamy, but deaf men often do not marry (Nyst, 2007:28).

The social and economical status of the deaf in the community varied as with the hearing people within the community. Some were prosperous and of high status, while others were poor. E.S. and L.P. told us that their father was rich, had a big house, and married 5 wives. His financial situation was such that he donated food to poor families. Y.Z. (male, 65), on the other hand, reports that his family was poor and had a hard life.

An important difference between deaf and hearing boys concerned education and literacy. Deaf boys did not attend school, as there were no special educational settings for deaf students. Consequently, all deaf men were monolinguals in AJSL and illiterate (as is not unusual situation in other village communities; see e.g., Marsaja 2008:77 for a similar situation in Desa Kolok), which was a constant source for anguish and frustration. The older deaf boys usually worked and helped with the family's livelihood, while younger boys just stayed at home: "In Algeria I played with a rope and a football and that was all. It bored me terribly. There was no deaf school. It was boring and I sat outside and wandered around all the time, playing marbles" (Y.Z.). Most girls, both deaf and hearing, did not attend school, but rather stayed at home and helped their mothers with the housekeeping. In that respect, then, hearing and deaf girls were alike.

\subsection{The sign language that developed in the community, AJSL}

As we can learn from the description in Briggs and Guède, in the 1950s there was a sign language in the community, used by both deaf and hearing 
members. We do not know when this language emerged and whether it was influenced by other sign languages. One of our interviewees, M.A., a man 84 years of age, told us that his grandmother was deaf. E.S. and L.P. had a deaf father, who died in 1995, at the age of 96 . We can deduce, then, that deafness in the community runs at least for five generations, some 110 years ago. Although it is impossible to give an exact estimation of the age of the language, it is clear that it was passed down for at least three generations, as six of our interviewees had deaf parents and one had a deaf grandmother. Importantly, both deaf and hearing members were involved in the process of acquiring and transmitting the language. As for possible contacts with other sign languages and signing systems, since deaf children did not go to school, the educational system could not have been the source for the sign language. It is possible that there were deaf in the Muslim population of Ghardaia, but we have found no information whatsoever about that. To the best of our knowledge, there are no dictionaries or any other documentation of Algerian Sign Language, so it is impossible to establish any relationship (or the lack of it) between the two languages. ${ }^{3}$ The only relevant piece of information regarding possible influence from a sign language used by Algerian Muslims was provided by U.B, a deaf man, 55 years old, who immigrated to Israel from Morocco. U.B. has a Muslim friend from Algeria, and he also has friends who use AJSL. He says the signs used by his Muslim friend are different from those of his friends who are AJSL signers.

Whatever the source of the language might be, it is clear that it served as a main means of communication in families with deaf members, as evidenced in the following vivid description: E.S. (female, deaf, 55):

"My mother signs AJSL to this day...Every time my aunt and my mother would talk about different issues and gossip, I would cry. After they would eat and my aunt would go home, my mother would call me over and say: 'Let me tell you about all the different things that your aunt told me - about her quarrel with her husband, and about how her husband refused to give her money'. It was good for me to hear these things from my mother, because we are very close and she tells me everything. This connection with my mother is very important to me. My mother shares with me everything from her heart."

L.P. (female, 60) points out that "The whole family from Ghardaia knows AJSL excellently and the hearing have full command over AJSL, exactly like the deaf."

The language was not confined to the family unit. L. P. reports that "In my neighbourhood in Ghardaia we had Arab neighbours and we always spoke in AJSL. ${ }^{4}$ They knew our language. But outside the village the situation was absolutely different, not the same. Only the neighbours know and recognise 
that this is local AJSL... my uncle knows AJSL very well." While this description raises the possibility of influence from the signing of deaf Muslims, we don't have any positive evidence for that. As pointed out above, we haven't been able to find any information about Algerian Sign Language, apart from the fact the language used by the Algerian Deaf community today is related to French Sign Language. It is not clear, though, whether the language in use today is related to any signing systems that were used by deaf people in the M'zab area during the first half of the $20^{\text {th }}$ century.

We do know, however, that there were deaf individuals in two other towns in the area: Laghouat and Aflou. Laghouat is $280 \mathrm{~km}$. north of Ghardaia, and Aflou is $80 \mathrm{~km}$. north-east of Laghouat. We interviewed two people from Aflou and one from Laghouat. All three people have other deaf members in their families, and all three are AJSL users. Apparently, people from the three towns visited each other, and as mentioned above, we learnt that some families from Ghardaia moved to these towns, probably in search of better livelihood. It may well be that some of these families had deaf members, but we do not have any information about that as yet.

Deaf-born children acquired AJSL from their deaf family members. When deaf children were born to hearing families, they acquired the language from deaf adults in the vicinity - extended family members, neighbours or friends, or hearing family members who knew how to communicate in sign language. Hearing people who married deaf people acquired the language from their spouses if they did not know it previous to marriage.

S.S., a hearing woman (age 80) who married a deaf man, describes how she learned to sign: "I can hear and I speak and understand the language well. Once I did not know how to use AJSL and now I know...my husband was deaf. He taught me AJSL and I began to learn and grasp the signs slowly. I did not work outside the home. I was only at home..."

It is interesting that in this signing community, some deaf members acquired AJSL not from older deaf language models, but from fluent hearing signers, a situation very different from urban signing communities in which deaf children often acquire the language from deaf peers. Although Y.Z.'s mother was hearing, she learned AJSL to communicate with her deaf children, so they acquired it naturally from her as well as from other community members. Z.M., who is deaf, was not exposed to AJSL at home. She acquired the language from interactions with Y.Z.'s (hearing) mother: "In the beginning I looked at Y.Z. and his brothers but I did not understand one word of AJSL. Y.Z.'s mother loved me very much. I looked at how she signed and I learnt the sign language from her. Now our communication is good." These instances illustrate that hearing people played an important role along with deaf members of the Ghar- 
daia Jewish community in the acquisition and transmission of this language. Signing hearing people in Ghardaia, then, were multilinguals; they used AJSL with the deaf members of the community, and they used at least one spoken language, the local Arabic dialect. Men also studied Hebrew as the language of prayer and religious rites, and French as the language of commerce. The deaf people in the community were monolingual in AJSL.

In summary, the picture that emerges is that there was no 'deaf community' per se in Ghardaia; rather, the deaf were part of the entire community. This is characteristic of many villages where a sign language developed (see Meir et al 2010 and references there). The deaf people living in Ghardaia were integrated in the community in many ways. Communication was accessible, they held ordinary jobs, were married to hearing spouses, and enjoyed an economical situation that was similar to their hearing counterparts. Their life was very similar to the lives of the hearing members of the community. However, there are two respects in which deaf people differed from hearing people, as pointed out above. The first is education: deaf boys did not attend schools while hearing boys did. Thus, the critical skills of reading and writing were denied them, damaging their ability to participate in Torah reading and later to accommodate to life in Israel. Second, deaf people always married hearing spouses, whereas hearing people were not restricted in their choice of spouse. These socio-linguistic characteristics changed drastically when the community emigrated from Algeria.

\section{The use of AJSL in Israel}

Between the years 1943 and 1962 the entire Jewish community left Ghardaia and immigrated, mainly to Israel and to France. The first wave of immigration was between 1943-1950. Due to growing tension between Berbers, Muslims and Jews in the M'zab area and in Algeria in general, 500-600 Jews immigrated to Israel and France (Briggs and Guède, 1964). In 1950-1951 the tensions in Algeria diminished to some extent and the Jews stopped leaving Algeria. Some immigrants who were unsatisfied with life in Israel returned to the M'zab region at that time (from the archives of Beit HaTfutsot \#73772).

In the $1950 \mathrm{~s}$, a second wave immigration began, motivated both by the establishment of the State of Israel in 1948, and by the Algerian War of Independence with France. The Jews of the region were regarded as French allies, and as such they felt increasingly unsafe in their homes and began to leave, again. The last wave of immigration from Algeria to Israel was in 1962. No Jews remained in Algeria today. 
The emigration from Algeria changed the life of the community members in every aspect of life. First and foremost, the community itself disintegrated. Part of the community immigrated to France while the other part moved to Israel. According to Nagel (2004), the majority of the wealthier Jews moved to France, while less affluent members of the community moved to Israel. Those who moved to Israel settled in different places in the country. Thus, members of the Ghardaia community no longer shared a physical location, and consequently the close-knit relationships between the community members collapsed.

Second, the immigrants had to adjust to many changes, among them the fact that the Jewish society in Israel was, by and large, secular, while the Ghardaian immigrants were observant Jews. They had to learn a new language, Hebrew, to find housing and to find jobs. Many families had had to sell all their property in order to raise the money for the trip to Israel, and came to Israel penniless. All of our interviewees describe their first years in Israel as years of poverty and hardships.

For the deaf members of the community, the immigration entailed further changes, the two most significant were the educational system for deaf children and the encounter with the emerging Deaf community in Israel, and its language, Israeli Sign Language (ISL). The educational system for the deaf in the 1950s and early 1960s consisted of several schools, nursery schools and special classes for the deaf (in Jerusalem, Tel Aviv, Haifa, Beer Sheva and Nazareth Illit, Plaut 2007). The schools in Jerusalem and Tel Aviv were boarding schools with dormitories. There was also a vocational rehabilitation center in Tel-Aviv. The Ghardaian deaf immigrants met, for the first time in their lives, deaf people who went to school and could read and write. Though the immigrants joined the deaf schools in Israel, those who were already in their teens found the studies in school very frustrating; they were not accustomed to the discipline and learning habits required in school. Moreover, they had to learn a new spoken language, Hebrew. As a result many of them left school after a few years and did not attain an adequate level of literacy. A vivid description of the experience was narrated by Y.Z.:

"My friends and I attended Niv, the school for the deaf. I began to learn how to go to school. I slowly understood how to find my way into school. I sat quietly with a group of new immigrants. I am from Algeria, one immigrant was from Egypt and two were from Romania. All of us came here. The class consisted of a mixed audience. I stayed in the class and studied. My mother made an effort for me to study at the school for the deaf. I studied with four other friends in my class. I was a new immigrant and studied but I did not fully understand how to write on the blackboard A-B-C-D...". 
Upon entering the educational system, the new immigrants encountered another sign language, ISL. Though the schools for the deaf adhered to an oral approach, the children used sign language among themselves (Meir and Sandler 2008). ISL, although a very young language at the time (about 20-30 years old), served as the common sign language for deaf people who came from different countries and different backgrounds.

The second major factor influencing and changing the lives of deaf immigrants from Ghardaia was the Deaf community in Israel. As mentioned in the introduction, the Deaf community in Israel developed in the late 1930s. Its members came from different backgrounds, both in terms of their country of origin, and in terms of their language. A few were born in Israel, and some of them went to the school for the deaf in Jerusalem that was founded in 1932, but the majority were immigrants who came to Israel from Europe (Germany, Austria, France, Hungary, Poland), and later on from North Africa and the Middle East. Some of these immigrants brought with them the sign language of their respective communities. Others had no signing, or used some kind of home sign. ${ }^{5}$ These deaf individuals started looking for other deaf, and formed small social groups that began to meet on a regular basis, creating the founding group of the Deaf community in the country. This burgeoning group attracted more deaf people. In 1943 the temporary committee of the association was set up and the following year the Association of the Deaf was officially founded. The association building that was built by 1958 was used as place for social gatherings of the deaf, and as a place where group activities and trade courses were provided.

The meetings with other deaf people eventually drew the immigrants from Algeria to the Deaf clubs. This was a very big change in their life style. In Ghardaia (as in other towns of North Africa at the time) there were no Deaf clubs. The seminal social unit was the family. Going to a social gathering in a club was something very new, and at times threatening, to people coming from a very conservative community. This was especially true of young girls. Both M.S. and E.S. recount that it was very difficult to persuade their fathers to let them go to the club. In the case of E.S., it was only after a respectable deaf man from the Israeli community made a promise to look after her that her father granted her the permission to go to the club.

At the Deaf club, the young Algerian immigrants met educated deaf people. The difference between the educated and non-educated members of the community was widely felt those days:

"One member, who had arrived at the time of the establishment of the state and had learned Hebrew well, tells how occasionally, while recounting a story, he would find himself stumped over the lack of a sign and would 
have to use speech. In such cases, only the educated could understand. To get the meaning across to the others who could not lipread, it was necessary to compose whole stories, and create a situation in which the meaning would be brought home." (J. Shunari 1969;4, in Meir and Sandler 2008;195)

The ISL community members came from many different countries in Europe, Africa, and Asia, but the AJSL users nevertheless stood out as a distinct group. The AJSL signers shared their place of origin, a sign language, the North African Jewish tradition and ways of life, and they were illiterate. These characteristics singled them out, and, according to their own recollections and feelings, not favourably. Z.M. says:

"It is said that people of Algerian origin are dangerous. It is said that we are vengeful and stubborn, but this is not true. I prefer to use AJSL in private conversations on the side and not in front of everybody, so that the other deaf people don't get offended or say that we are dangerous. I would like to explain to them about Algeria very much, although people have classified the Algerians in a negative way. Now I speak the sign language naturally. I am not ashamed of my sign language. Everyone has their own natural language."

Within the Deaf community, it seems that the most salient characteristic of this group was their language, AJSL. Since they felt stigmatised, and they felt that their language was "responsible" for singling them out, they tried to avoid using it when ISL signers were around, as is evidenced in Z.M.'s quote above. E.S. also notes that "When speaking in AJSL, we speak in secret. The other deaf people looked and asked what it is: 'Is the AJSL secretive?' I told them that this is just the way it is. This is the Algerian Sign Language." Thus, AJSL users who associated with other deaf people became bilingual in sign: they used ISL to communicate with members of the Deaf community, and kept AJSL for private, family settings. Crucially, AJSL remained the only means of communication with their hearing family members: E.S.: "I communicate only in ISL with friends at all times, but when I talk to my mother, I immediately switch to AJSL. I talk with my deaf sister in ISL but with my mother mainly in AJSL and sometimes in ISL. Every Saturday the whole family comes to visit my mother. There are two hearing brothers, one hearing sister, and five deaf brothers and sisters, and we all communicate in AJSL with a few word signs in ISL - a mixture of ISL and AJSL." The choice between using AJSL or ISL became an 'act of identity' in the sense of Le Page \& Tabouret-Keller (1985). By choosing to use ISL in the public sphere, AJSL users signalled their identity as members of the Israeli Deaf community. Their Ghardaian identity, displayed by the use of AJSL, was confined to the family setting. 
In spite of the strong sense of inferiority and stigmatization, deaf Algerian individuals became integrated into the Deaf community, and most of them married deaf spouses of non-Algerian origin. Of our 7 deaf interviewees, only two married a deaf Algerian spouse. ${ }^{7}$ This marriage pattern resulted in a shift towards ISL within the nuclear family, as the non-Algerian spouses usually did not learn AJSL. The children born to these families grew up with ISL, and indeed their competence in AJSL is very weak: L.P.: "My eldest son knows quite enough AJSL but my other sons can't communicate in the language." E.S.: "I used to speak with my sisters in AJSL at all times, but today it is different because of my children (who only know ISL). Sometimes, depending when, we still speak in AJSL." Both Y.Z. and M.S. have deaf children, but they cannot sign or understand AJSL.

It seems, then, that the immigration to Israel entailed drastic changes in the social structure of the community and consequently in their patterns of language use. The community disintegrated, and deaf people found themselves drawn to social networks that were based on deafness rather than on family ties. This shift also brought about a change in language use. Deaf Ghardaians were exposed to ISL, and became bi-lingual in sign. Because of the stigma associated with AJSL, it disappeared from the public sphere and was restricted to communicating with family members, especially hearing family members, who remained monolingual in sign as they were not exposed to ISL.

The integration with the Israeli Deaf community brought about changes in marriage patterns: AJSL users married members of the Deaf community, usually of non-Algerian origin. Therefore, AJSL is not used in the new families that were established in Israel, and the language is not passed on to the younger generation. According to various scales of language endangerment, a language that is not passed down to a younger generation is moribund (Krauss 2001) or dying (Hudson and McConvell 1984, Fishman 1991). It seems then, that if nothing drastic happens, AJSL will disappear with the current generation of users. However, importantly, in the past few years AJSL users feel that they would like to use the language more often. This might be the result of two processes: first, the feeling of stigmatization has waned over the years as AJSL users became part of the Deaf community, and many of them who grew up in Israel from an early age went to school, acquired literacy, and hold jobs. Second, they may also feel that the language is endangered, and that measures should be taken to preserve it and the heritage that it represents. As Z.M. points out: "The history and geography of Algeria is very interesting. In the future our heritage will disappear, AJSL will disappear and it is a shame. Very few people speak in AJSL." 


\section{The survival of AJSL in Israel alongside ISL: what makes AJSL different?}

As we mentioned in the introduction to this chapter, most of the deaf immigrants who came to Israel and brought with them another sign language or signing system did not maintain their original sign language, and switched to use ISL. As the people themselves testify in our interviews with them, they do not remember the lexicon of their original language. This could be regarded as a case of $L 1$ attrition, the decline in native language proficiency among immigrants (see inter alia Köpke et al 2007 and references there). In case of the Israeli Deaf community, the decline was rather final, as the immigrants stopped using their L1, and eventually forgot it. Among these immigrants, AJSL users stand apart; they did not forget their L1. Though most of them use ISL as their main language of communication in everyday life, they still remember AJSL and can hold a conversation in it. AJSL seems to be much more durable, as it continued to exist in Israel alongside ISL for about 50 years (however, its vitality is diminishing now, as we point out at the end of this section). What may explain it?

The notion of Ethnolinguisitc Vitality (EV) seems relevant here; AJSL exhibits much more EV than other L1 sign languages. This notion was first introduced by Giles, Bourhis and Taylor (1977), when trying to characterise ethnolinguistic groups in terms of their ability to maintain distinctive collective identity in intergroup settings. They define EV as "that which makes a group likely to behave as a distinctive and active collective entity in intergroup situations" (p.306). They propose that there is a correlation between social and psychological factors and linguistic behaviour when ethnic groups come into contact. Dimensions such as institutional support, control over resources, social status, demographic strength (related to sheer numbers of ethnolinguistic group members as well as to their distribution throughout a particular territory) all contribute to the likelihood of an ethnolinguistic group to survive as a distinct group in intergroup settings.

The notion of EV has received a considerable amount of attention over the years (see e.g. the recent volume of Journal of Multilingual and Multicultural Development, 2011, volume 32;2 that was devoted to EV). However, it has hardly been applied to sign languages. The only analysis of the vitality of a signed community based on the notion of EV is Judith Yoel's (2007) analysis of L1 attrition in Russian deaf immigrants in Israel. Based on a model of EV developed by Allard and Landry $(1986,1992)$, Yoel suggests that the attrition of Russian Sign Language in Russian deaf immigrants in Israel (evidenced by their difficulties in two naming tasks) is related to 
various sociological, sociopsychological and psychological factors. For example, in the Former Soviet Union (FSU), residential schools served as central locations for groups of deaf people, providing them with social clubs, sports clubs and job opportunities. Such locations played an important role in creating group identity and ample opportunities for daily communication. In Israel, deaf immigrants from FSU were dispersed across the country, sometimes in peripheral areas with very few other deaf people. This resulted in a drastic weakening of their demographic and economic status, lack of opportunities to interact with other Russian deaf immigrants and a decrease in the status of their language and identity as a group. All these contribute to the fact that their L1, RSL, is losing grounds to the dominant sign language in the country, ISL.

We would like to apply the notion of EV to analyse the socio-linguistic situation of AJSL users in Israel in order to explain its relative vitality to other L1 sign languages. We adopt a model developed by Landweer (2000) for analyzing potential viability of languages of Papua New Guinea. She suggests eight factors which are indicative of the direction a speech community takes with respect to the maintenance of or shift from its traditional language. We present these factors and explore whether and how they can be implemented with respect to AJSL.

The first factor is related to the relative position on the urban-rural continuum: the more rural the community is, the less it is likely to be in contact with other languages, and therefore the vitality of its language is stronger. Regarding AJSL, the language arose in an urban setting, within the city of Ghardaia. Yet within this urban setting, the community was isolated from the surrounding Muslim community. According to our interviews, Jewish deaf individuals did not have regular contact with other deaf people, and their language developed and thrived as it was not threatened by contact with another sign language. This might suggest that the relevant factor is the degree of social isolation rather than the geographic isolation or remoteness, although more information about other sign languages in the area is needed to support this claim.

In Israel, the situation has changed drastically. The Ghardaian community disintegrated, and most of the deaf members came into contact with deaf people using another sign language, ISL, and became sign-bilingual. It could be expected that AJSL would lose ground to ISL. However, this process is much slower than could have been expected, as many AJSL signers still use the language today.

A second factor has to do with population and group dynamics: there should be a critical mass of fluent users of a language for it to survive, 
though the exact numbers may vary in different situations. Regarding AJSL, it is difficult to assess the number of people who use it. As documented by Briggs and Guède, in 1960 there were 25 deaf people in the community. Yet the number of AJSL users was higher than that, since many hearing family members used the language as well. We do not know how many of the deaf immigrated to Israel. Furthermore, we have no demographic statistics regarding the number of deaf people of Algerian origin in Israel, nor do we know how many hearing people use the language. But the role of the hearing people in expanding the number of AJSL users is critical. Deaf immigrants from other countries did not have a substantial number of hearing people as part of their linguistic community.

The hearing members of the AJSL community played additional roles in other factors as well. Landweer (2000) refers to frequency and type of codeswitching: frequent individual unbounded code-switching is regarded as the most threatening form of bilingualism to the vitality of a language, whereas a community with a majority of members with monolingual allegiance is the least threatened. The hearing AJSL signers remained monolingual in sign even in Israel. ${ }^{8}$ The deaf AJSL users met other deaf people and another sign language when the immigrated, and therefore many of them became bilingual, and some of them use ISL in many more communication domains than their use of AJSL. But the hearing family members did not go to Deaf clubs and were not exposed to ISL. They remained (sign) monolingual in AJSL. Therefore, deaf AJSL users had to continue to use AJSL with the hearing family members. There was no other way for them to continue the rich and natural inter-family communication they were used to in Ghardaia.

Another important factor in determining the ethnovitality of a language has to do with domains in which the language is used: languages that are used for communication in more domains in life (cultural events, social events, home, education) are stronger than languages used in fewer domains. AJSL is used in one domain that other sign languages brought by immigrants were not used in: the nuclear and extended family. Deaf people from other countries often remark that they did not use sign language with the hearing members of their families - parents, siblings, aunts and uncles etc. They used sign language to communicate with other deaf people, mainly in social gatherings in the Deaf club. These social circumstances offered intensive language contact between the languages brought by immigrants of different countries. The immigrants did not have a domain where they could or should have used their L1. For AJSL signers, the nuclear and extended family provided such a domain, thus increasing the vitality of the language. In addition, it also enriched the social networking of AJSL users, since the 
members of this language community were related to each other not only by social acquaintance but also by family ties, and many of them knew each other, maintaining closed-knit social networks (cf. Milroy 1980).

AJSL does not fare so well on the following two indicators: (a) language prestige: a language that has prestige among other languages in the region or country has greater potential for use in the foreseeable future; and (b) access to a stable and acceptable economic base: a language that benefits its users economically has greater potential for survival.

AJSLwas not prestigious, and it did not have any economical advantages. As indicated by the quotes in section 6, deaf Algerian immigrants were uneducated, and felt inferior to other members of the Deaf community in Israel. We can infer from their reports that they felt that other deaf people looked down on them, so much so that they were hesitant to use their language in the presence of other deaf people. As for economic benefits, sign languages in general do not provide strong economic basis for their users, as most professions require the knowledge of the ambient spoken language. But when compared to other sign languages in Israel, any resources available in sign, such as vocational courses and use in the educational system, are provided solely in ISL.

Internal and/or external recognition of the language community as a separate entity within the larger community also contributes to the strength of the language. AJSL offers an interesting perspective on this issue. It is clear that AJSL users were marked as a distinct group within the Deaf community in Israel, characterised both by their ethnic origin and by their language. However, this group identity was not perceived as a positive feature. The group and its language were looked down upon. Nevertheless, despite its negative connotation, the language seems to have served as a marker of group identity, possibly supporting the group's sense of identity and promoting the continuous use of the language. The lesson to be learnt from AJSL is that a language need not be perceived as a positive characteristic of a community in order to function as a supporting factor in reinforcing ethnolinguistic vitality.

By examining AJSL within the framework of EV, we gain unique insights into the factors involved in the endangerment and maintenance of sign languages. It seems that there are two main factors that contributed to the vitality of AJSL as L1 relative to other L1 sign languages among deaf immigrants to Israel. The first is that the language served as the main means for communication within the family unit. This had several implications. For one, the AJSL community was larger than the number of deaf people in the community. Hearing signers significantly increased the number of people using the language. Second, AJSL continued to be a useful and central 
means of communication even after the Ghardaia community disintegrated, since the family continued to serve as a vital social unit in the new country. Third, the hearing AJSL users remained sign-monolingual. Those community members who became bilingual (the deaf signers who became part of the Deaf community in Israel) had to use their L1 with their hearing family members. Therefore, the linguistic community had enough monolinguals in its new surroundings for the language to survive the contact with the dominant sign language, ISL. This analysis, then, highlights the crucial role that the hearing signers played in preserving the language's vitality.

Immigrants from other countries were in a different socio-linguistic situation. In Europe, deaf children were often sent to boarding schools (for example, there was a famous school for the deaf in Wissensee, Berlin; see Biesold 1993). From interviews with people who grew up in Europe (see endnote 1), we learn that they used sign language with their deaf peers, not with their hearing family members. In Israel they met with deaf people from different countries, and hence their L1 could no longer serve as a means for communication, and therefore they stopped using it. People who immigrated to Israel from North African countries such as Morocco and Egypt often had deaf siblings, with whom they presumably communicated in sign. It might also be the case that some of the hearing family members used signing to some degree to communicate with their deaf family members. But from what little we know about these signing systems, they seemed to be more like extended homesign systems, that is, a communication system that emerges within a family with deaf members, and is restricted to that family. We know of no community who used a sign language except for the Ghardaia-M'zab community. Therefore people from these other countries had very few individuals to communicate with in sign, and once they became part of the Deaf community in Israel, they acquired and switched to ISL.

The second factor that contributed to the relative vitality of AJSL is that it served as a characteristic of a group, and therefore strengthened the feelings of group identity among its members. As pointed out above, interestingly, this characteristic was not perceived as positive or prestigious, yet it helped in strengthening bonds within community members. AJSL was something they "did" when they were on their own, something they did not wish to share with others.

AJSL managed to survive in Israel for over 50 years in close co-existence with ISL, despite not being prestigious or of economic benefit. Our study provides unique insights into the endangerment of sign languages. It indicates that the family and the community play an important role in contributing to the vitality of the language. Moreover, it shows that hearing signers 
can be a major factor in the vitality of a sign language. It also shows that in-group cohesion can be maintained even when the language they use is not prestigious.

With these understandings in mind, we can turn from the past and present to the future: What is awaiting for AJSL in the near future? Unfortunately, the future does not seem to hold great promise to AJSL, because the factors that contributed to its survival are diminishing. There are less and less hearing family members who still use AJSL. The majority of hearing family members who were born and grew up in Israel adapted to the general attitude of hearing people towards signing in the country, namely that it is something that belongs to deaf people. So AJSL is less and less used in families. As mentioned in section 6, most deaf AJSL users marry people of non-Algerian origin, so they do not use the language with their spouses, and consequently it is not used with the younger deaf generation. The language, then, is not passed down to a new generation. Finally, Algerian signers are much less stigmatised nowadays, and they feel less inferior and marginalised. The consequences of this positive development are that they are more fully integrated into the Israeli Deaf community, and hence are more likely to use ISL rather than AJSL.

Yet there are two points of hope. First, there are also AJSL users in France. It might be that the circumstances there are somewhat different, and that the language is still passed on to children. The AJSL community in France should be the focus of future studies, as it will provide a more complete picture about the language and its vitality. Second, it is our hope that the study and the documentation of the language, which is currently in its initial stages, will help to raise more awareness of the language, and this in turn might have some positive effect on its vitality. 


\section{Sara Lanesman and Irit Meir}

\section{Acknowledgements}

The results reported in this paper are based on Sara Lanesman's MA thesis, written under the supervision of Irit Meir and Ulrike Zeshan. The research project was supported by ISF research grant \#553/04 to Irit Meir, and ISF research grant \#580/09 to Irit Meir and Wendy Sandler. It is also part of the EuroBABEL 08-EuroBABELFP-007 project, headed by Ulrike Zeshan. Information regarding members of the ISL community of non-Algerian origin is based on interviews with 33 ISL signers which were conducted during 2004-2008, as part of a research project titled "The birth and development of a language: The sign language of the Deaf community in Israel”, supported by an ISF grant \#553/04 to Irit Meir.

\section{Notes}

1. This information is based on interviews with 33 ISL signers of different background, which were conducted during 2004-2008, as part of a research project titled "The birth and development of a language: The sign language of the Deaf community in Israel".

2. The first author is a member of the ISL community.

3. For details, see the sociolinguistic sketch of AJSL, this volume.

4. When referring to "Arab neighbours", L.P. means those Arab people living close to the Jewish quarter. No Muslims lived inside the Jewish quarters.

5. For a description of the history of the Deaf community in Israel and the development of ISL, see Meir \& Sandler (2008).

6. The term "Algerian Sign Language" is used by E.S., though it refers to AJSL. The term AJSL was coined by the authors. As mentioned above (section 5.2), we do not know whether AJSL is related to Algerian Sign Language.

7. Notice that this marriage pattern was different from that of Algeria. In Algeria, deaf people married hearing people. In Israel, deaf-deaf marriages are the norm.

8. A similar situation is described in Dikyuva, Panda, Escobedo, \& Zeshan (this volume) with regard to Mardin Sign Language. 


\section{References}

Allard, R. \& R. Landry

1986 Subjective ethnolinguistic vitality viewed as a belief system. Journal of Multilingual and Multicultural Development 7(1). $1-12$.

Allard, R. \& R. Landry

1992 Ethnolinguistic vitality beliefs. In W. Fase (ed.), Maintenance and loss of minority languages, 171-95. Amsterdam: John Benjamins.

Aronoff, M., I. Meir, C. A. Padden \& W. Sandler

2008 The roots of linguistic organization in a new language. Interaction Studies: A Special Issue on Holophrasis vs. Compositionality in the Emergence of Protolanguage 9(1). 131-150.

Biesold, $\mathrm{H}$.

1993 The Fate of the Israelite Asylum for the Deaf and Dumb in Berlin. In R.Fischer and H. Lane (eds.), Looking Back: A Reader on the History of Deaf Communities and their Sign Languages, 157-169. Hamburg: Signum.

Briggs, L C., \& N. L. Guède

1964 No more forever: A Saharan Jewish town. Cambridge, Mass.: The Peabody Museum.

Fishman, J.A.,

1991 Reversing Language Shift. Clevedon, England: Multilingual Matters.

Giles, H., R. Y. BouD. rhis \& Taylor

1977 Towards a theory of language in ethnic group relations. In H. Giles (ed.), Language, Ethnicity and Intergroup Relations, 307-348. London: Academic Press.

Goldin-Meadow, S.

2003 The resilience of language: What gesture creation in deaf children can tell us about how all children learn language. New York: Psychology Press.

Groce, Nora E.

1985 Everyone here spoke sign language: Hereditary deafness on Martha's Vineyard. Cambridge, Mass: Harvard University Press.

Hudson, J. \& P. McConvell

1984 Keeping language strong: Report of the pilot study for Kimberley Language Resource Centre. Broome: Kimberley Language Resource Centre. 
Jacobs, M.

1967 The isolation of a 1,000-year-old Saharan trading town. American Anthropologist, New Series, Vol. 69(2). 220-222.

Kastner, I., I. Meir and W. Sandler

(in preparation) Kfar Qasem Sign Language.

Kisch, S.

2000 Deaf discourse: The social construction of deafness in a Bedouin community. MA Thesis, Tel-Aviv University, Israel (in Hebrew).

Kisch, S.

2007 Disablement Gender and Deafhood among the Negev Arab-Bedouin. Disability Studies Quarterly 27(4). Accessed December 5, 2007 at http://www.dsq-sds.org/handle/osul. dsq/1208533130.

Kisch, S.

2008 The Social Construction of Deafness in a Bedouin Community in the Negev. Medical Anthropology 27(3). 283-313.

Köpke, B., M.S. Schmid, M. Keijzer \& S. Dostert (eds.).

2007 Language Attrition: Theoretical perspectives. Amsterdam: John Benjamins.

Krauss, M.,

2001 Mass language extinction, and documentation: the race against time. In Osamu Sakiyama (ed.) Lectures on Endangered Languages: 2 - from Kyoto Conference 2000 (Endangered Languages of the Pacific Rim C002). Osaka: Osaka Gakuin University.

Landweer, M. L.

2000 Indicators of ethnolinguistic vitality. Notes on sociolinguistics 5.1:5-22. Accessed February 2012 at http://www.sil.org/sociolx/ ndg-lg-indicators.htm.

Le Page, R. \& A. Tabouret-Keller.

1985 Acts of identity:creole-based approaches to language and ethnicity. Cambridge: Cambridge University Press.

Marsaja, I. G.

2008 Desa Kolok - a deaf village and its sign language in Bali, Indonesia. Nijmegen: Ishara Press.

Meir, I., \& Sandler, W.

2008 A language in space: The story of Israeli Sign Language. New York: Lawrence Erlbaum Associates 
Meir, I., Sandler, W., Padden, C., \& Aronoff, M.

2010 Emerging Sign Languages. In M. Marschark and P. Spencer (eds.) Oxford Handbook of Deaf Studies, Language, and Education, Volume 2, 267-280. Oxford: Oxford University Press.

Milroy, L.

1980 Language and social networks. Language in Society 2. Oxford: Blackwell.

Nagel, R. L

2004 Jews of the Sahara. Einstein Journal of Biological Medicine 21: $25-32$.

Nyst, V.

2007 A descriptive analysis of Adamorobe Sign Language (Ghana). Utrecht: LOT.

Padden, C. A., I. Meir, W. Sandler \& M. Aronoff

$2010 \quad$ Against all expectations: Encoding subjects and objects in a new language. In D. B. Gerdts, J. C. Moore \& M. Polinsky (eds.), Hypothesis A/Hypothesis B, 383-400. Cambridge, MA: MIT Press.

Plaut, A.

2007 The history of educational frameworks for students with hearing impairments in Israel 1932-2005. The Mofet Institute [in Hebrew].

Sandler, W., I. Meir, C. A. Padden \& M. Aronoff

2005 The emergence of grammar: Systematic structure in a new language. $P N A S$ 102(7). 2661-2665.

Seliger, H. W. \& R. M. Vago.

1991 First language attrition. Cambridge: Cambridge University Press.

Yoel, J.

2007 Evidence for first-language attrition of Russian Sign Language among immigrants to Israel. In Quinto-Pozos, D. (ed.), Sociolinguistics in Deaf Communities, Volume 13, Washington D.C.: Gallaudet University Press. 


\title{
Signing in the Arctic: External influences on Inuit Sign Language
}

\author{
Joke Schuit
}

\section{Introduction}

Even a linguist who strongly opposes the views of linguistic relativism would probably agree that the environment in which a language is used may exert some influence on that language - however marginal that influence may be. It is likely to be no coincidence, for instance, that Dutch has many words related to water. This is probably due to the geographic setting, as the Netherlands lies below sea level and has both a long coast line and many inland areas of water. This is also reflected in the many proverbs and sayings in Dutch that are related to water, a few examples are given in (1).

(1) a) Water naar zee dragen.

'To do something superfluous' (lit. to carry water to sea)

b) Het water staat hem tot aan de lippen.

'He's up to his neck in difficulties.' (lit. the water reaches his lips)

The aim of this chapter is to describe several external influences on Inuit Sign Language (IUR). IUR is the language used by deaf Inuit, who live in the Canadian territory of Nunavut. In this chapter, the geographical situation is of particular importance to IUR, while the other external influences, (i.e. the demographic situation, the gestures of the wider community, and the language contact situation) are also significant with respect to other sign languages. Obviously, there are more factors that might influence a language, but these are outside the scope of this chapter. Influences that are prominent in the IUR context are here reflected upon more broadly, in terms of how they affect other signed and spoken languages.

This chapter is organised as follows. In the second section of the introduction, IUR is introduced, followed by the methodology. Then in section 2, I focus on the physical environment, and discuss geographical influences on the spoken and signed languages of the Inuit. Section 3 addresses the influence of the demographic environment on spoken and signed languages, 
and I show that IUR is comparable in this respect with village sign languages. Section 4 discusses gestural influences from the wider community on sign languages in general, and on IUR specifically. Influences from language contact on sign languages are addressed in section 5.1, and in section $5.2 \mathrm{I}$ describe how the sociolinguistic situation impacts IUR. A descriptive conclusion with suggestions for further research is provided in the final section (6).

\subsection{The Inuit and Inuit Sign Language}

Inuit Sign Language, or Inuit Uukturausingit in Inuktitut (abbreviated as IUR), is the language used as the main means of communication by deaf Inuit $^{1}$ in Nunavut, Canada. Inuit are the native people of the Arctic, and different bands live from Alaska to Greenland. It is possible that IUR is used among deaf people in other regions as well, but the focus of my research has been on Nunavut (see Figure 1 below).

Nunavut is Canada's largest territory (about 2 million $\mathrm{km}^{2}$ or 787,000 square miles), but has only 32,000 inhabitants who live in 25 communities spread throughout the territory (Census of Canada 2011, available online).

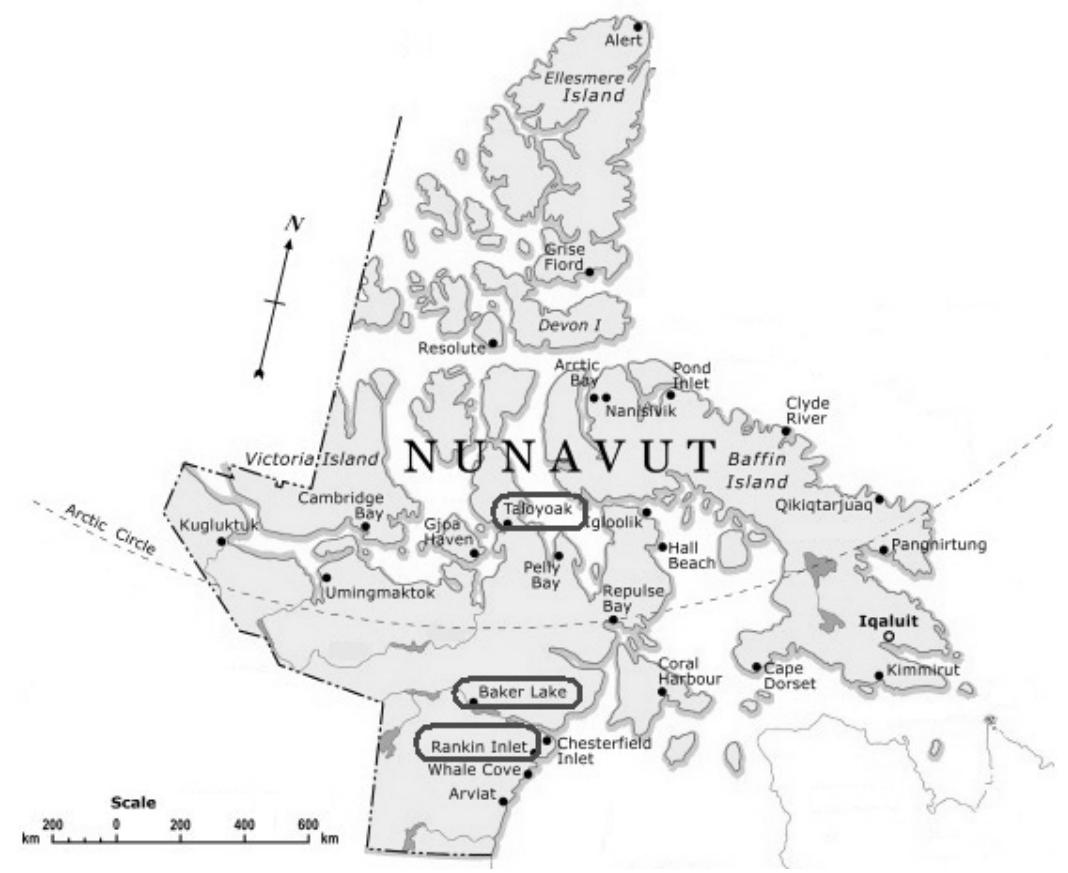

Figure 1. Map of Nunavut, with fieldwork locations indicated. 
Travel to and between Nunavut's communities is by airplane, as there are no roads between the communities. These flights are rather expensive, and face-to-face contact between deaf Inuit from different communities is scarce. IUR is thus used in an extraordinary sociolinguistic setting: for about 47 deaf signers ${ }^{2}$ (MacDougall 2000), it is the sole means of communication, but these people live in separate communities spread across the immense area. It is difficult to give an estimate of the number of hearing signers, especially since it differs from community to community. I estimate there are at least two hearing signers to each deaf signer, who have learned IUR because they are in close contact with a deaf IUR signer.

IUR is not used in a single village community like the other sign languages described in this volume. The geographical spread of the sign language is somewhat reminiscent of Plains Indian Sign Language (anonymous reviewer). But the similarity ends there, because Plains Indian SL is mainly used as an alternate sign language by hearing people (Davis 2010), while IUR is only used by deaf people. In the past, however, hearing Inuit used IUR to communicate across different dialects (Olsthoorn 2010), and possibly also to trade with other peoples. Effects of this widespread sociolinguistic setting are described in section 2 below.

The Inuit traditionally were nomadic hunter-gatherers (inter alia Wachowich 1999). For this reason, MacDougall (2000) suggests that the origins of IUR could be similar to those described for Aboriginal people in North and South America and Australia. Sign languages in those communities developed as alternate communication systems used during hunts, and/or as lingua francas to bridge mutually unintelligible languages or dialects (MacDougall 2000). Indeed, signs were used in Inuit culture as early as the 18th century to communicate among different bands (Olsthoorn 2010). Due to unknown reasons, a rather high percentage of Inuit were born deaf or became deaf. The signs already existing in Inuit culture probably were used with the deaf children, and then evolved into a language.

At the present time in Nunavut, varying degrees of subsistence hunting exist, although dogsleds and spears are no longer used. Snow mobiles, rifles, and shotguns have made hunting easier. In the first part of the 20th century, the Inuit lived nomadically, and different bands could be found from Alaska to Greenland. It is possible that IUR is used in other areas of the Arctic as well, but further travel is outside the scope of the current documentation project.

The spoken language of the Inuit is Inuktitut, which is a member of the Eskimo-Aleut language family. This family of languages is spoken in the Arctic region from the Aleutian Islands west of Alaska to Greenland in the 
east. The Inuit branch has many dialects, of which both Dorais $(1990,2010)$ and Harper (2003) describe four groups, each named for its region: Alaskan Inupiaq, Western Canadian Inuktun, Eastern Canadian Inuktitut, and Greenlandic Kalaallisut. Inuktitut itself has several subdialects (see Dorais 2010 for an overview), but it is unclear as yet whether these have had distinct influences on IUR, so I will not account for them here.

Inuktitut is a highly polysynthetic language, which allows for grammatical morphemes to attach to a lexical stem. The examples in (2) below illustrate that the lexical stem is either nominal or verbal (indicated in bold), and that grammatical morphemes can be polymorphemic, i.e. denoting more than one meaning, as -tara in (2.b) which indicates that the first person (1s) sees (as the verb is taku-) a third person (3s), in this case the dog (qimmiq).
a) Arna- u- junga.
Woman-be- INTR.PART.1s
'I'm a woman.' (Johns 2007:544)
b) Qimmiq taku- lauq- tara. Dog see- DIST.PAST- TR.PART $1 \mathrm{~S} / 3 \mathrm{~S}$
'I saw the/a dog.' (Johns 2007:546)

Inuktitut

Inuktitut will be of importance in section 5.2 about language contact.

In the current documentation project, the focus is on Nunavut, Canada's Arctic territory, where IUR is currently used as the predominant language by roughly 47 people (MacDougall 2000, but see also note 2). Because deaf, monolingual IUR signers live in different communities, interactions are rare and lexical variety abounds. They seem to have no difficulties understanding each other, however.

The prevalence of deafness in Nunavut is considered to be 5.7 in 1,000, an incidence that is almost six times higher than in southern Canada (StamosDestounis 1993 in MacDougall 2000). This led MacDougall to estimate that there are about 155 deaf people in Nunavut; most were identified in Nunavut's communities, and many of them use a sign language. Those who use sign language are generally surrounded by a network of family and friends who also sign. MacDougall (2000:13) found "little or no evidence of 'social stigma' associated with deafness in the communities [...] and there was no apparent social exclusion because of deafness".

About two-thirds of the deaf Inuit use American Sign Language (ASL) or Manually Coded English ${ }^{3}$ (MCE), while the remaining one-third use IUR. Also, a few deaf Inuit are bilingual in ASL and IUR (MacDougall 2000). The use of ASL as opposed to IUR is mainly related to formal education. Formally-educated deaf Inuit aged over 40 attended residential schools for 
the deaf in southern Canada where ASL was used. Some of these people do know some IUR signs, but do not use the language regularly. Deaf Inuit children nowadays attend the mainstream school in their home community, with the aid of a qualified ASL interpreter, and do not know IUR. This is another contributing factor to the endangerment of IUR. In fact, IUR is only used as a primary language by those deaf individuals who have not been to school, or only attended school for a short period.

\subsection{Methodology}

The author collected data for documentation and description purposes, which is the underlying aim of her project. Data collection is aggravated by the fact that it is difficult to find out which of the many communities in Nunavut are home to deaf IUR users. The three communities visited, i.e. Rankin Inlet, Baker Lake and Taloyoak (see Figure 1 above), were chosen because of familiarity with the deaf IUR users there. The following sections provide information on these three communities and the respective informants.

\subsubsection{Participants}

In Rankin Inlet, the data were collected from two deaf men and one hearing woman. PU is deaf, in his early forties and bilingual in IUR and ASL. He has three deaf and five hearing siblings. The language used in the family is a combination of ASL, fingerspelling, and some IUR with the elders. PU learned IUR from the age of 12 from YS, a deaf man now in his late sixties who grew up with a deaf brother (deceased), and about five hearing siblings (one brother survives). YS is monolingual in IUR, but as he and PU have been friends for over 25 years, he has learned some fingerspelling and uses some ASL signs. Both signers recognise which signs are from IUR and which are from ASL, and consider these languages as different but equal. Both men are skilled artists and work in a ceramic workshop. The hearing informant SS, wife of YS, was only able to take part in two recording sessions. Other family members of PU and YS did not participate in this documentation project due to various personal reasons.

In Baker Lake the data come from one deaf and one hearing man, both in their early forties. BS was deafened at the age of seven and therefore acquired Inuktitut and English as first languages. From the age of seven he learned IUR, which is now his main means of communication. He does not use spoken or written Inuktitut and English. He has no deaf relatives. Since 
the early 2000s, BS has also learned some ASL, and some IUR signs have now been replaced by their ASL counterparts. DK is a hearing friend of BS, and has been using IUR with him since they were in their mid-teens. DK also speaks Inuktitut and English, and often interpreted BS's signing for the researcher. As BS also acquired English and Inuktitut as a child, he uses more mouthings than the other deaf informants. Both men in Baker Lake also recognise the different signs of IUR and ASL, but in contrast to the informants in Rankin Inlet, they consider ASL to be more elaborate than IUR, although not necessarily better.

Three other deaf adults live in Baker Lake, as well as one deaf child, who is related to PU from Rankin Inlet. Allegedly, two of the adults use IUR, and the third uses ASL. The researcher met one of the deaf adults who uses IUR, and found her using ASL signs also. This might be due to the researcher not being Inuk. The lady was too shy to participate in the project.

In Taloyoak, the informants are from one family. JU is the only deaf member in the family. His hearing wife, MU, is bilingual in IUR, Inuktitut and English, and therefore also acted as interpreter for the researcher. An overview of the participants' characteristics is provided in Table 1.

Table 1. IUR study: Participants' characteristics

\begin{tabular}{|c|c|c|c|c|c|c|}
\hline Location & Informant & Gender & $\begin{array}{l}\text { Hearing } \\
\text { status }\end{array}$ & Age & $\begin{array}{l}\text { Deaf } \\
\text { relatives? }\end{array}$ & Languages \\
\hline \multirow[t]{3}{*}{$\begin{array}{l}\text { Rankin } \\
\text { Inlet }\end{array}$} & PU & male & deaf & $\begin{array}{l}\text { early } \\
40 \mathrm{~s}\end{array}$ & $\begin{array}{l}3 \text { deaf } \\
\text { siblings }\end{array}$ & IUR, ASL \\
\hline & YS & male & deaf & $\begin{array}{l}\text { late } \\
60 \mathrm{~s}\end{array}$ & $\begin{array}{l}\text { deaf } \\
\text { brother }\end{array}$ & IUR \\
\hline & SS & female & hearing & $\begin{array}{l}\text { late } \\
50 \mathrm{~s}\end{array}$ & $\begin{array}{l}\text { (deaf } \\
\text { husband) }\end{array}$ & $\begin{array}{l}\text { IUR, Inukt., } \\
\text { Engl. }\end{array}$ \\
\hline \multirow[t]{2}{*}{$\begin{array}{l}\text { Baker } \\
\text { Lake }\end{array}$} & $\mathrm{BS}$ & male & deaf & $\begin{array}{l}\text { early } \\
40 \mathrm{~s}\end{array}$ & --- & $\begin{array}{l}\text { IUR (Inukt., } \\
\text { Engl.) }\end{array}$ \\
\hline & DK & male & hearing & $\begin{array}{l}\text { early } \\
40 \mathrm{~s}\end{array}$ & --- & $\begin{array}{l}\text { IUR, Inukt., } \\
\text { Engl. }\end{array}$ \\
\hline \multirow[t]{2}{*}{ Taloyoak } & JU & male & deaf & $\begin{array}{l}\text { late } \\
60 \mathrm{~s}\end{array}$ & --- & IUR \\
\hline & MU & female & hearing & $\begin{array}{l}\text { late } \\
50 \mathrm{~s}\end{array}$ & $\begin{array}{l}\text { (deaf } \\
\text { husband) }\end{array}$ & $\begin{array}{l}\text { IUR, Inukt., } \\
\text { Engl. }\end{array}$ \\
\hline
\end{tabular}


All participants have travelled outside of their home community a number of times. Most have travelled within Canada. They all live among non-Inuit people, as not all community members are Inuit. All communities are visited by tourists, and all informants have been in contact with people from abroad. Whether these visitors were signers is unknown.

\subsubsection{Data collection procedure}

Data in this chapter was collected during three fieldwork trips. In March and April of 2009, seven weeks were spent in Rankin Inlet and one week in Baker Lake. In May and June of 2010, three weeks were spent in Rankin Inlet as well as three weeks in Baker Lake. Three succeeding weeks in Taloyoak had to be cancelled due to informants' personal reasons, so an extra trip was planned in August 2010. Due to financial reasons, the researcher could spend only ten days in Taloyoak.

Data recording in Rankin Inlet was done in the large kitchen of Kivalliq Hall, the campus building of Nunavut Arctic College. In Baker Lake, recording took place either in the home of one of the two informants or in the apartment where the researcher was staying. In Taloyoak, the recordings were made in the home of JU and MU. The researcher was present during all recording sessions, but sometimes left for a few minutes. These minutes, still recorded on camera with consent from the informants, showed that the language used in absence of the researcher was not different from the language used in her presence.

Data come from an unstructured interview setting. The researcher usually asked some general questions about Inuit culture, often via a bilingual informant. This led the informants to narrate stories about past and present life. In Rankin Inlet, the monolingual informant YS was dominant in the conversation. In Baker Lake both informants were equally dominant, as in Taloyoak.

In 2009, the bilingual informant PU translated what the monolingual informant YS signed into (a form of) Manually Coded English ${ }^{4}$ for the researcher, and often also added information. This was also recorded on video. A year later, this translation was no longer necessary, as the researcher understood most of the IUR stories of YS. Furthermore, all recorded data was watched with PU a couple of days after the recording session, allowing the researcher to clear up any uncertainties. In Baker Lake during both fieldwork trips, the bilingual informant DK translated what the monolingual informant BS signed into English for the researcher. During both fieldwork trips, the 
translation was necessary, as the researcher was not able to understand all of the IUR of BS. This was partly due to the large number of Inuktitut and English mouthings, and partly due to the first visit in 2009 being rather short because of financial limitations. In Taloyoak, the interview was in English with MU who translated some questions into IUR for JU so that he was able to participate somewhat. The researcher had not taken into account that questions intended for JU could be answered by MU, his wife. Instead of translating the questions, she simply answered them. Because of the short time spent in Taloyoak, not much data was recorded, but there was enough for the purposes of this chapter.

During the fieldwork trips, over 17 hours of material were recorded. As mentioned, this includes the translations and additional information of PU in Rankin Inlet in 2009, of DK in Baker Lake in 2009 and 2010, and of MU in Taloyoak in 2010. About six and a half hours of video material have been translated, of which two and a half have been fully annotated in ELAN. This includes data from all three communities. The remainder of this chapter presents findings from the translated data. First, geographical influences on the spoken and signed language of the Inuit are examined.

\section{Geographical influences}

When discussing geographical influences in the context of Inuit Sign Language, it is almost impossible not to mention the famous claim that the Inuit language has hundreds of words for 'snow'. In various forms, this idea has circulated for many years in numerous linguistic, anthropological and popular science publications. Martin (1986) describes how this notion can be traced to Whorf (1940/1956), who used it as an illustration of how languages differ in their classification of the environment:

"We have the same word for falling snow, snow on the ground, snow packed hard like ice, slushy snow, wind-driven flying snow - whatever the situation may be. To an Eskimo, this all-inclusive word would be almost unthinkable; he would say that falling snow, slushy snow, and so on, are sensuously and operationally different, different things to contend with; he uses different words for them and for other kinds of snow." (Whorf 1940/1956:216)

Pullum (1991) argues that this alleged proliferation of terms is a "hoax", referring to Martin's (1986) research. She claims that Inuit languages actually have only two root terms for 'snow', while Huhn (2004), on the other hand, claims that "the Eskimo snow vocabulary example is no hoax" (137-8). 
Dorais (1990) and Kaplan (2003) list about two dozen lexemes for 'snow' and 'ice' in two different Inuit languages. Kaplan (2005) concludes by saying that it should not be surprising that the Inuit pay attention to features of their landscape, as this is crucial for survival. Therefore their language has labels for the different occurrences of snow and ice. ${ }^{5}$

Examples like this one can be found in many languages of the world, mostly within the lexical domain. Concepts that are relevant to a people are lexicalised in the language, while the same concepts may be irrelevant for others and are therefore not lexicalised in other languages. While influences of social environment on language structure have occasionally been described for sign languages, to date, no study has addressed the possible influences of climate and landscape. I could speculate how climate, landscape and foliage could influence a sign language, but as their communities differ in many additional domains, it would be tricky to argue that a specific feature, for instance, a larger signing space, results from a warm climate.

Nonetheless, when looking at IUR, the influence of the environment seems an appropriate factor to take into account. The Inuit live in an Arctic environment where weather conditions are extremely cold. Body parts, in particular the extremities, suffer when exposed to the weather. Since sign languages rely on the use of these extremities i.e. the hands, arms and face, it is interesting to investigate how the climate affects the language. It might be expected that signs are formed in a smaller space, to reduce the loss of body heat. One might also expect a smaller set of handshapes, as people usually wear mittens, which would prohibit distinctions between the fingers. Thus one might predict only handshapes that differ in the opening and closing of the hand (a flat, open hand versus a fist, for instance), and/or in thumb extension. However, this is not what occurs.

A preliminary study of IUR handshapes has revealed the use of at least 27 different ones. A thorough phonological analysis has not been conducted as yet, so it is not known which of them are in fact phonemic. It is clear, however, that IUR has a set of phonetic handshapes that is at least as large as that of Adamorobe Sign Language (AdaSL), a sign language used in the village of Adamorobe in Ghana. AdaSL has 29 phonetic handshapes (Nyst 2007), of which at least 15 are identical to those of IUR. Although the set of IUR handshapes is small compared to the 70+ handshapes of the Sign Language of the Netherlands (NGT) (Van der Kooij 2002), it seems unlikely that this disparity can be attributed to the cold climate given that AdaSL is used in Ghana.

This is not to say, however, that the Arctic climate does not have any influence on IUR. Informants reported that messages tend to be short when 
communicating outside in cold weather. People wait until they are back inside a house before expanding the conversation. Only absolutely necessary information is transmitted outside. The climate therefore seems to have affected IUR mainly pragmatically, but not phonologically. As it was not possible to interview people outside due to the cold, the exact nature of this shortening of messages is unknown. For this same reason, no signs for 'snow' or 'ice' were elicited, as research regarding these terms would have had to have been done outside. Pictures or movie clips would not be sufficient to show all the differences in snow or ice conditions which may be encountered in the Arctic. The difference between hard and soft snow on the ground for instance, is not clearly visible in a picture. In spontaneous texts, weather conditions were hardly ever discussed.

Another important factor that has influenced IUR is the fact that the language is used in a large area. As mentioned above, the Inuit of Nunavut are spread across the territory in 25 communities. Because of the distances involved, deaf Inuit only have contact with those who happen to live in the same community. In the past however, the Inuit lived a nomadic life, travelling across the Arctic. When nomadic life was abandoned, the extensive contact between people from different regions decreased considerably (Condon 1983; Wachowich 1999), and face-to-face contact between deaf monolingual signers today is rare. As a result, one can now detect lexical variety in IUR. MacDougall (2000) describes how approximately one-third of the signs in his spontaneous data corpus was found to be different, but one of his informants reported that these were still "easily understandable". An initial aim of the current project was to determine the lexical variation between communities, but methodological problems prevented the researcher from performing either a comparative analysis or a frequency count. Firstly, eliciting specific signs was regarded as inapt: direct questioning is considered inappropriate in Inuit culture (Rasing, in prep.). Secondly, using the spontaneous data to perform this task was not feasible, as these data differ too much with respect

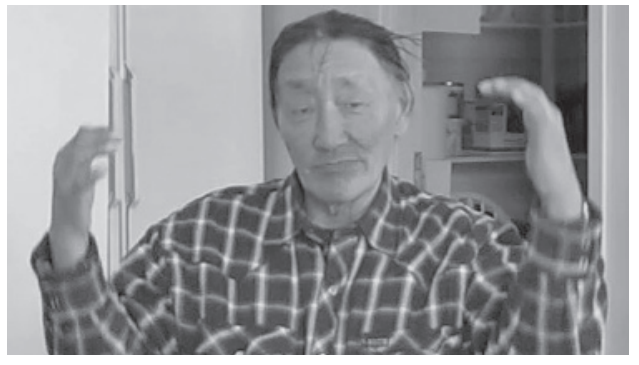

The sign CARIBOU in Rankin Inlet

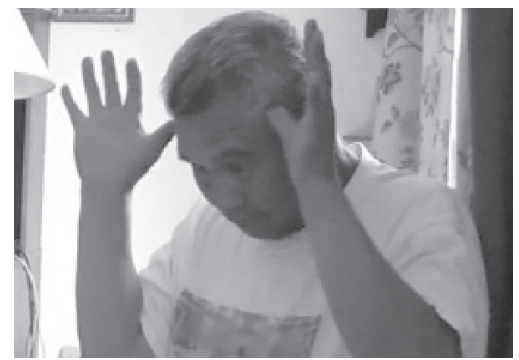

The sign CARIBOU in Taloyoak

Figure 2. Lexical variety for the sign CARIBOU. 
to content among the three communities. One observation that could be made clearly, however, is that signs for animals differ among the communities. Due to the high iconicity of these signs, people have little trouble understanding each other. Figure 2 shows two variants of the sign CARIBOU. Each of the signs uses the same synecdoche, i.e. the antlers of a caribou, but how this is incorporated in the sign differs, as should be clear from the pictures.

The following section explores how a language community can further influence a language.

\section{Demographic influences}

The size and composition of a community can affect its language. For spoken languages, Kusters (2003) and Lupyan and Dale (2010) describe how language complexity can be related to community size: small communities with many first language speakers have morphologically more complex languages compared to large communities with many second language speakers. For sign languages, it seems to be different: village sign languages, i.e. sign languages used in small communities, have different structures than urban sign languages, i.e. sign languages with a larger Deaf $\operatorname{community}^{6}$ (see also other chapters in this volume).

Meier (2002) argued that sign languages are more uniform because of the possibilities the visual-manual modality affords them. When village sign languages were studied, however, their structures were found to be rather distinct from those of urban sign languages. Some of these differences were associated with the sociolinguistic situation of village sign languages; these communities share several socio-cultural characteristics. All communities have a high incidence of deafness, and a considerable number of hearing people sign. Usually, there is no Deaf community and the sign language is endangered, often by the national sign language (Nyst, 2012). Some or all of these demographic aspects may have influenced various village sign languages. Washabaugh (1986) argues that the lack of a Deaf community resulted in the divergence between ASL and Providence Island Sign Language, the latter of which he describes as highly context dependent and immature.

Nyst (2007) suggests that AdaSL is strongly influenced by the high percentage of hearing signers. She argues that the virtual absence of entity classifiers in AdaSL is related to the co-speech gestures used by the hearing people: they do not make use of the observer perspective, so neither do deaf people. The observer perspective occurs when a storyteller is in the role 
of narrator. The storyteller makes use of entity classifiers, which are handshapes that represent people, animals or objects, and can be used in motion verbs. For instance, an NGT signer could sign MAN PERSON-MOVES-FROM-LEFTTO-RIGHT, meaning 'a man walks from left to right'. The sign PERSON-MOVESFROM-LEFT-TO-RIGHT is an upright index finger that moves from left to right. The handshape, i.e. the upright index finger, is the entity classifier, in this case referring to the semantic class of people. The other perspective that we find is the character perspective, where the storyteller takes on the role of one or more characters. The observer perspective is used more often in Western Europe and among hearing people in their co-speech gestures.

Nyst (2007) suggests that the large proportion of hearing second language signers has influenced the structure of AdaSL significantly. For instance, AdaSL has relatively many iconic signs, as these are easier to learn for L2 signers. Also, non-native signers have a tendency to proximalise articulation, resulting in a larger signing space (Nyst 2007). AdaSL is thus different from NGT (and perhaps other urban sign languages) with respect to these features because of the large number of L2 signers.

These factors also affect IUR, which is the main means of daily communication for only 47 deaf people. At least twice as many hearing people use this language, so the vast majority of IUR users are second language signers. As Nyst (2007) suggested for AdaSL, this has influenced the structure of IUR. The handshapes for instance, are much laxer than observed in urban sign languages, and preliminary analysis indicates a rather flexible ordering of signs. The signs themselves have also been impacted by the large proportion of hearing signers. Like many sign languages, IUR uses manual and non-manual gestures, as well as borrowings from spoken languages (Inuktitut and English). The next section examines gestural influences in IUR and other sign languages, and the borrowings are discussed in section 5.2.

\section{Gestural influences}

Manual gestures that already exist in a population are often used in the sign language. This has been described for both urban and village sign languages. Wilcox (2004) shows how gestures can be the input of a sign language's vocabulary, by becoming either lexical content signs or grammatical function signs. An example of content signs (the main focus here) is the Dutch gesture for 'tasty', i.e. moving an open hand backwards and forwards next to one's head. This is used in NGT as well, with the same meaning (LEKKER 'tasty'). As the gesture in the hearing culture, the sign is restricted to food, 
although the word lekker (nice) can be used in other contexts as well. Another example, from a village sign language, is given by Nyst (2007). She describes the use of measure signs in AdaSL, which are not only used in the village of Adamorobe, but in wider African culture as well.

A study focusing on the gestures accompanying spoken English of hearing Americans shows that the head gestures used in this population are found in ASL as well, and McClave (2001) therefore suggests that this is also a type of linguistic borrowing. In addition, she describes how hearing Americans use gestural space to localise referents in space; for instance, an informant consistently used the same location to refer to one referent.

Non-manual gestures from the hearing culture are thus also used in the sign language, and have the same meaning. A headshake denotes negation in both Dutch hearing and Deaf culture, while the nod means affirmation. However, the non-manuals in NGT are grammatical markers, while in spoken Dutch, these are not grammatical (cf. Zeshan 2004). As described for other sign languages also, it is possible to negate a statement with a headshake in NGT (3), but not in Dutch (4).

$\frac{\mathrm{hs}}{\text { MAN HOUSE GO }}$

$N G T$

(4) ${ }^{*}$ De man gaat naar huis

Dutch

the man goes to house

'The man doesn't go home.'

In both Greek Sign Language and Turkish Sign Language, a backward head tilt denotes negation (Antzakas and Woll 2002 and Zeshan 2003, respectively), and this is a non-manual gesture used by the wider population in both countries.

Many non-manual gestures can be found in IUR. Inuit culture has several non-manual gestures; most often 'yes' and 'no' are indicated by facial gestures, although Inuktitut also has lexical items, $i i$ and $a h k a$ respectively (Kulchyski 2006). The non-manual gesture for 'yes' is a raising of the eyebrows and a widening of the eyes, while 'no' involves furrowing the brow, squinting, and wrinkling the nose (ibid). Kulchyski (2006) adds that the non-manual gestures might be accompanied by a smile or a frown. His research was carried out in the community of Pangirtung, but these non-manual gestures have also been observed in the authors' three fieldwork locations and in Greenland (Cornelia Buijs, Museum Volkenkunde (Ethnology), Leiden, p.c.). 
Figure 3 depicts the non-manual 'no', which is commonly seen in IUR. However, signers often add a headshake, as well as a manual sign. In fact, a short study of negation in IUR has shown that the non-manual negation marker, i.e. the wrinkled nose and lateral headshake combined, are not used without manual negation, as can be seen in (5). That is, it is not possible to negate a sign with non-manual features exclusively, as can be seen in (6) below.

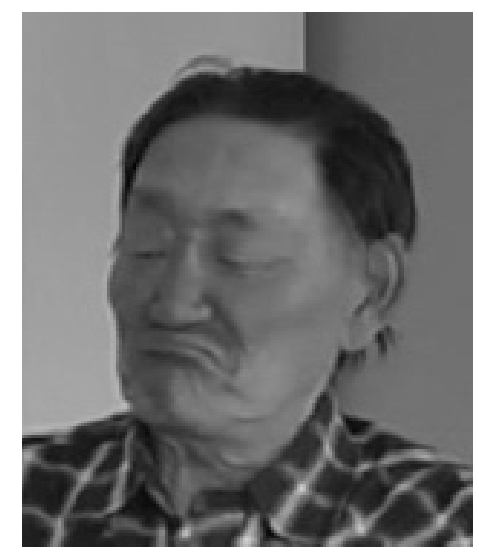

Figure 3. IUR non-manual 'no'.

(5) POLAR-BEAR SEE NEG ROUND-EARS ${ }^{\wedge}{ }_{\text {LONG-NAILS }}^{\wedge}$ ANIMAL $\frac{\text { neg }}{N E G}$
'I didn't see any polar bear, or any grizzly bears.'

(6)

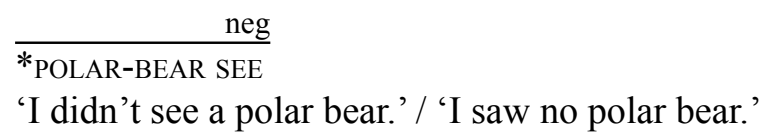

Also manual gestures from Inuit culture are incorporated into IUR. As in some other sign languages, the numbers 1 to 10 are derived from the gestures that hearing people use. For the IUR numbers 'one' to 'three', two synonyms are found. These variants are motivated by which finger is selected to express ONE. The first variant selects the little finger for ONE, as can be seen in the first picture in Figure 4, ONE-1. The subsequent numbers follow the first finger. In contrast, the other variant selects the index finger for the first number, as can be seen in the second picture in Figure 4, ONE-2. Though both variants are used regularly, the variant that selects the little finger is regarded as more traditional, and is the only option used in enumeration. In enumeration, the non-dominant hand takes on the function of buoy, while the dominant hand points to (and possibly touches) the fingers associated with the appropriate 
ordinal, e.g. the dominant hand points to the little finger to indicate 'first'. The other fingers may or may not be extended.

Both variants show a preference for the orientation pictured in Figure 4, with the palm of the hand toward the signer. Occasionally, the palm is away from the signer, and slightly toward the floor. This occurs most often when signers are trying to remember an exact number.

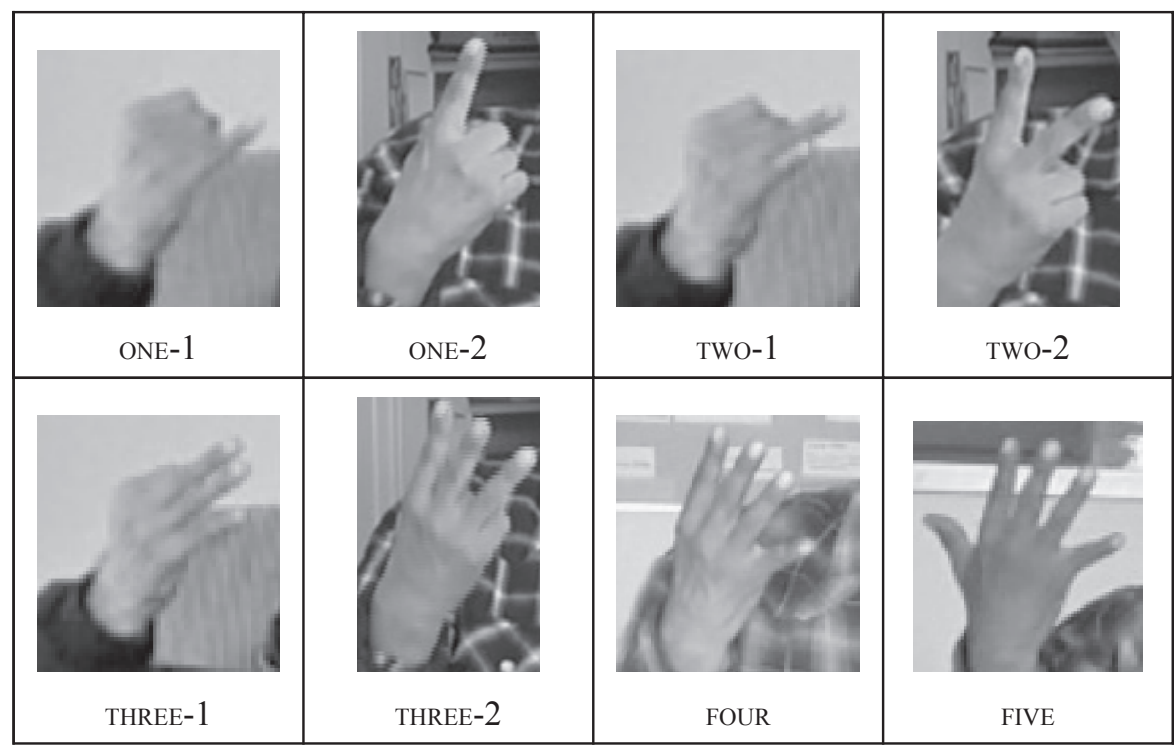

Figure 4. IUR numbers 1-5, with both variants for ONE, TWO and THREE.

Further research is needed to investigate whether there are more manual and non-manual Inuit gestures in IUR. Influences from Inuktitut are described in the section on language contact below.

\section{Language contact}

Languages in contact influence each other. A description of all possible outcomes of language contact is beyond the scope of this chapter. As borrowing is attested for in IUR, this is the main focus of this section. First, the different types of borrowings found in sign languages are discussed in section 5.1 about sign language contact. Then in 5.2, the language contact situation of IUR is described, and the borrowings are split into three different types: lexical items, loan translations, and mouthings. 


\subsection{Sign language borrowing}

Sign languages can incorporate borrowings in different ways. The most straightforward way is borrowing a sign from another sign language. As for spoken loan words, the borrowing is adapted to the borrowing language's phonological rules. Loan signs can be borrowed to fill a gap in the lexicon, or to replace an old sign. Sutton-Spence and Woll (1999) describe how British Sign Language (BSL) filled a gap in its lexicon by borrowing the sign CLASSIFIER from ASL. The ASL classifier handshape that denotes the class of vehicles, became, with an added movement, the BSL sign CLASSIFIER. Replacement borrowings can be found in many of the signs for countries, which are borrowed from the sign languages of those countries. For example, the current BSL signs JAPAN, DENMARK and THAILAND are borrowed from those countries, and the old signs for these are now obsolete (Sutton-Spence and Woll 1999).

Another form of sign language borrowing is a loan translation. This is a literal translation of the sign, or better said, of the meaning of the sign. This process is very common in name signs. BSL examples are the signs ICELAND and GREENLAND, which are composed of the signs ICE and LAND, and GREEN and LAND, respectively (Sutton-Spence and Woll 1999). Another example is the sign for the city of Islamabad in Indo-Pakistani Sign Language. a:ba:d means 'dwelling, settlement' in Urdu. The combination of ISLA:M 'Islam' and JAGAH 'place' forms the sign for Islamabad (Zeshan 2000). Loan translations are also found in village sign languages. AdaSL borrows from Akan, in which the sign HOUR represents hitting a bell. The Akan word $d J n$ means both 'hour' and 'bell' (Nyst 2007).

Fingerspelling is conventionally seen as borrowed from spoken language. As it has been part of many sign languages for a long time though, the relation with the spoken language is distant, and at least should be regarded as removed from spoken language, as it is in fact the representation of written language (Brentari and Padden 2001). The manual alphabet consists of various handshapes to represent the written letters of the alphabet. Not all sign languages have a manual alphabet, but each sign language that does, has its own manual alphabet, even where their surrounding cultures make use of the same written alphabet. For instance, ASL and BSL are used alongside the same written language, but use two totally different manual alphabets. ASL's alphabet is one-handed, while BSL uses a two-handed alphabet. A manual alphabet is used primarily to spell out written words. In some cases these fingerspelled words are abbreviated, and become akin to a lexical sign. 
Examples are the signs CLUB (c-b) in BSL (Brennan 2001), BLUE (b-l) in NGT and WOULD (w-d) in ASL (Brentari and Padden 2001). Some sign languages even allow abbreviated words to be incorporated into new handshapes. The NGT sign LUI (lazy) consists of a handshape that combines the NGT handshapes L, U, and I into one new handshape (all fingers extended, apart from the ring finger which is bent; middle and index fingers together). In LSQ, the sign ROI 'king' combines the three letters, again creating a new handshape (Miller 2001).

Additionally, the individual handshapes can also be adopted to form new signs in a process known as initialisation. Signs are initialised when the handshape of the sign refers to the first letter of the written word. For ASL, initialisation has been described as "one of the most productive of wordbuilding processes"(Brentari \& Padden 2001:104) since the handshapes of the alphabet can be used to systematically distinguish associated signs, for instance in GROUP, TEAM, and ASSOCIATION. These signs have similar movement and location, but their handshapes differ, referring to the letters $\mathrm{G}, \mathrm{T}$, and A respectively. For other sign languages such as NGT, the process is not as productive as in ASL, but this does not mean there is no initialisation in NGT.

Finally, a large part of borrowing from spoken languages happens through mouthings, which are mouth patterns derived from spoken language, resembling spoken words (Boyes Braem \& Sutton-Spence 2001). Over half of the NGT signs produced in both structured and spontaneous texts are produced in combination with a mouthing (Schermer 1990). Schermer (1990) divides what she called "spoken components" into three types. The first are spoken components that represent only part of a Dutch lexical item, as for example $k o f$ accompanying the sign KOFFIE (coffee) or $n$ with the sign NIET (not). Where spoken components are reduced, it is the first part of the Dutch word that is truncated. The second type is spoken components that "specify, complement or disambiguate the signs they accompany" (Schermer 1990:111). Examples are grazen (to graze) with the sign ETEN (to eat); kwam (came) with the sign KOMEN (to come); and amman with the sign SCHOOL (Amman is the name of the school). The last type consists of spoken components that have the same meaning as the sign. This category contains the largest number of utterances, for example huis with HUIS (house); avond with AVOND (evening); leven with LEVEN (to live); and rood with ROOD (red). Although mouthings are often thought to be less native to the sign language, or even 'too hearing', Schermer (1990) clearly proves that mouthings are part of the grammar of NGT, and are used in many contexts. 


\subsection{Inuit Sign Language and its contact languages}

IUR is in contact with languages from different modalities (signed and spoken) and from different morphological types (polysynthetic and isolating). Thus it can be expected that the language contact influences on IUR are myriad. This section relates some influences of ASL, Inuktitut, and English on IUR. As ASL is used in the same visual-manual modality as IUR, lexical items can be incorporated, but it is shown here that some have been adapted to fit IUR's phonological system. The different types of borrowings from spoken language described for other sign languages (see section 5.1 above) have been found in IUR as well. Interestingly, both Inuktitut and English are sources of loan translation in IUR (5.2.2), as well as of mouthings (5.2.3).

\subsubsection{Lexical items from $A S L$}

As briefly explained in the introduction, in the 1970s (and maybe before and after), deaf Inuit children were sent to schools for the deaf in southern Canada, mainly in Winnipeg, Edmonton and Vancouver. The language of instruction ranged ${ }^{7}$ from sign language (ASL), to a sign system (Manually Coded English), to spoken English. Through contact among deaf Inuit, ASL has influenced IUR. The reverse might be true also for those deaf Inuit who use ASL as their main means of communication, but no research has been conducted from that angle. Most borrowed signs from ASL are recognised by signers as such.

ASL is used in the same modality as IUR, making incorporation of lexical items possible. These signs are adapted to the phonological system of IUR. The amount of borrowing differs from signer to signer. When analysing the borrowings, the ASL signs of bilingual deaf signer PU were considered as the basis, since he is the main model for ASL signs of the other deaf IUR informants. As his conversations with the researcher were also recorded (see section 1.2.2), his ASL forms could be analysed closely.

Some lexical borrowings seem to be signs for which no lexeme existed in IUR. An example is the sign HOME, which is borrowed in IUR without the movement. ${ }^{8}$ Other examples are the signs BOY and GIRL. The sign WATER is also borrowed, and used alongside the native IUR sign WATER (see Figure 5 for some illustrative examples). A reason people gave for borrowing the ASL sign WATER is that ASL signs are more easily understood by tourists who come to Nunavut. The truth of this is rather dubious, but nevertheless, apparently some deaf signers assume that ASL signs are widely known among hearing Canadians. 


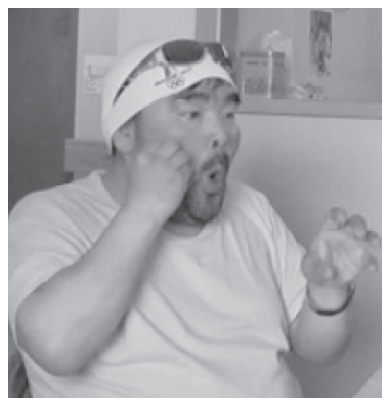

a) HOME

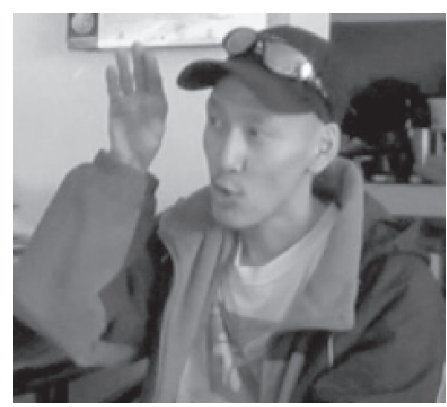

b) BоY (hand closes twice)

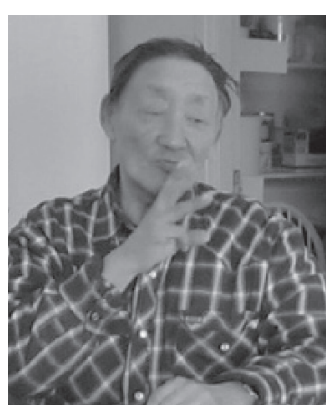

c) WATER

Figure 5. IUR signs borrowed from ASL.

ASL numbers SIX to NINE and the hand alphabet are also borrowed into IUR, but are used with limitations. Numbers are mainly used to refer to years, e.g. 1967. For counting and enumerating, IUR numbers are used (see section 4). The IUR numbers SIX to NINE are all two-handed, while the ASL equivalents are one-handed. Both are pictured in Figure 6.

\begin{tabular}{|l|l|l|l|l|}
\hline Number & Six & Seven & Eight & Nine \\
\hline In IUR & & & & \\
& & &
\end{tabular}

Figure 6. Numbers six to nine in IUR and ASL (ASL pictures cropped from http://lifeprint.com/asl101/pages-signs/n/numbers.htm)

IUR itself has no manual alphabet, probably because there was never a need to spell Inuktitut words (remember there is no education in IUR). Since many monolingual deaf IUR informants are illiterate, they do not use the ASL hand alphabet to spell out words. But manual letters are used to refer to certain people and places. It can be argued that some place names are borrowings from ASL, however. For instance, the ASL sign WINNIPEG is signed with an ASL W-handshape (index, middle and ring fingers extended) with a turning motion at the wrist. In IUR, the W simply stands for Winnipeg. It is likely 
that this is a borrowing from ASL adapted to IUR, i.e. deleting the movement. For other place names, there is no ASL sign, and for those the first letter of the place name is used: B for Baker Lake, R for Rankin Inlet. The bilingual signer PU and other deaf Inuit ASL signers would sign B-L and R-I. Tentatively, I would propose that since these place names are a nonnative phenomenon, the signs referring to them are non-native as well, especially since other toponyms have lexical signs, which are described in the following section.

\subsubsection{Loan translations}

Several loan translations from both Inuktitut and English have been established. As mentioned above, these are signs in which the meaning of words or their components is translated into an IUR sign. An example is the sign QALLUNAAQ, 'white man', which is a compound of EYEBROW and BELLY. It is a widespread assumption that the Inuktitut word qallunaaq is a compound of qallu 'eyebrow', and naaq 'belly'. Inuktitut does not allow compounding in this way, but many Inuit believe this to be true. Indeed, Dorais (2010:88) states that Qallunaaq means 'outstanding eyebrows'. The sign QALLUNAAQ is thus a loan translation, but of a misconception.

Other loan translations can be found in toponyms, or place names. Toponyms often have an Inuktitut name and an English equivalent, making it hard to determine which language the loan translation is from. A clear example from Inuktitut is the sign for Nunavut's capital Iqaluit. The place name means '(place of) many fishes', and the sign IQALUIT is the same as the sign FISH. An example from English is the literal translation for the Back River, which uses the sign BACK (which refers to a person's back) and RIVER. Some Inuit refer to this river as Ukkuhikhalik, meaning 'a place to find stone for making pots and oil lamps' (Nunavut Parks website). These are clearly different meanings, which makes it easier to conclude that the base of the loan translation in IUR is the English name.

A possible loan translation from Inuktitut can be found in the signs RED and BLOOD. In Inuktitut, aupaluktuq ('red', lit. 'it is blood-coloured') is derived from auk ('blood'). In IUR, the sign RED is identical to the sign BLOOD. It should be noted, however, that in other languages, the term for 'red' is also derived from or the same as 'blood', as for instance in Mian (Fedden 2011) and Warlpiri (Reece 1970), to name a few. 
Also there is similarity in how 'grey' is described in IUR and Inuktitut. In IUR, this is signed BLACK DARK LITTLE 'a little black'. In Inuktitut, similar strategies are found to denote grey, as qirnajuktuq literally means 'it tends to be blackish'. Other colour analogies however are non-existent. IUR has only two colour terms, namely BLACK and RED, while Inuktitut has two basic colour terms ('black' and 'white') and five other colour terms that are derived from nouns (like the word for 'red'). Other village sign languages in general have at least signs for 'black' and 'white', in line with Berlin and Kay's (1969) proposed universals for colour terms. This has been attested for the sign languages of Providence Island (Washabaugh 1980), Ban Khor (Nonaka 2004), and Adamorobe (Nyst 2007), as well as for Al-Sayyid Bedouin Sign Language and Kata Kolok (de Vos 2011). IUR is thus an interesting exception with respect to colour terminology (see Schuit (in prep.) for more information).

\subsubsection{Mouthings from English and Inuktitut}

The last type of borrowing attested for in IUR is mouthings. As IUR is in contact with at least two spoken languages, i.e. Inuktitut and English, it is not surprising that mouthings from those languages are found. IUR uses some English mouthings that accompany borrowed ASL signs. The aforementioned borrowing of HOME takes the mouthing of 'om', and some of the borrowed numbers take mouthings as well. However, English mouthings can also be found with IUR signs, and they follow the patterns Schermer (1990) found for NGT, discussed in section 5.1 above. Examples are TWELVEo'CLOCK with a mouthing of 'twelve'; 'airport' with the sign AIRPLANE; and 'time' with the sign TIME.

Some mouthings come from Inuktitut, but they are rare. This can be explained by the polysynthetic nature of Inuktitut, and the fact that roots hardly ever occur in isolation. Therefore, borrowing a mouthing that refers to one word, which could be mapped onto a single sign, seems problematic. Shortening of words, as described for NGT KOFFIE (coffee) above ('kof' as mouthing) might occur, but the researcher's knowledge of Inuktitut was too limited to do extensive research with respect to this question. Some examples are found nonetheless, for instance the sign FAR-AWAY, which is accompanied by a mouthing of patua ('far away'), and SNOW-ON-THE-GROUND, accompanied by aputi ('snow on the ground'). 
It seems odd that IUR borrows mouthings from both English and Inuktitut, as those two languages have very different morphologies typologically. But IUR is also used by many hearing Inuit, who are in close contact with a deaf Inuk, and most speak both Inuktitut and English. Indeed, analysis of the semi-spontaneous corpus data indicates that hearing signers use mouthings from both languages while signing IUR. Deaf monolingual signers are likely to have copied some of these mouthings from their hearing friends and family.

It is clear that each of the languages IUR is in contact with has had its individual influence on IUR. Borrowings from ASL occur alongside and in addition to IUR signs, and some loan translations from Inuktitut and English are found. Mouthings from Inuktitut and English occur with both IUR signs and borrowed signs.

\section{Conclusion}

Languages are influenced on many levels. In this chapter, the focus has been on the geographical and social environment in which languages, particularly IUR, exist. I have explained how the climate affects the use of IUR since communication outside tends to be brief, and how the geographical spread of the communities has influenced this language's lexical variety. The large proportion of hearing second language signers has influenced the structure of IUR in that handshapes are lax, and the order of signs is rather flexible.

Cross-linguistic studies have found correlations between the social and morphosyntactic structure of spoken languages (Kusters 2003; Lupyan and Dale 2010), an effect that seems to be reversed for sign languages (Washabaugh 1986; Nyst 2007): while small, isolated communities seem to have structurally more complex spoken languages, they seem to have structurally less complex sign languages. Both these correlations seem to hold for the Inuit community: their spoken language Inuktitut is polysynthetic and has strict morphological and syntactic rules. IUR on the other hand, seems to have a flexible syntax, and few morphological rules.

Both manual and non-manual gestures used in a hearing community can be incorporated into a sign language, and it is therefore perhaps unsurprising that gestures used by hearing Inuit are used in IUR. I described how IUR numbers come from the Inuit manual gestures, and how the non-manual gestures for 'yes' and 'no' are found in IUR as well. 
Finally, I described three different types of borrowing, which also occur in IUR. Lexical items are borrowed from ASL, and adapted to fit IUR's structure. Loan translations in IUR can come from both English and Inuktitut since it is possible to translate the meaning of a word from either language into IUR. Also, mouthings from both spoken languages can be found in IUR.

As the research regarding demographic influences on sign languages is scant, it would be apt to study the effects of community size on these languages cross-linguistically. However, it is hardly possible to control for other effects, like the high proportion of hearing second language signers in village communities (c.f. this volume). However, one may be able to find urban settings with large and small Deaf communities, and in those cases, the situations would be more comparable. Also, it would be interesting to study the amount of pointing to actual locations in urban sign language. Absolute pointing has been described in several village sign languages (see for instance Nonaka (2007) for Ban Khor Sign Language, and Marsaja (2008) for Kata Kolok) but remains under-researched in urban sign languages. In IUR, pointing is used abundantly as well, for example to refer to people and locations. Pointing is also used to denote the colour 'white', probably because there is always something white to point at, which is perhaps also a reason for not needing many signs for 'snow'.

\section{Acknowledgements}

This research was funded by a grant from the Amsterdam Center for Language and Communication, the Hans Rausing Endangered Languages Program - ELDP grant IGS0078, and by a Student Research Award of the Association for Canadian Studies in the Netherlands. I thank the Research Group Circumpolar Studies, Anne Baker, Roland Pfau, and three anonymous reviewers for comments on an earlier version of this chapter. This research could not have taken place without my informants in Nunavut, to whom I will be forever grateful. 


\section{Notes}

1. It should be noted that the term Eskimo though still in use in Alaska, has fallen out of favour in Canada and Greenland for referring to the people who live there. Only the language family continues to be called 'Eskimo', and this is the only use of the term 'Eskimo' in this chapter, except where other authors are quoted directly. Instead, the people will be referred to as Inuit (or singular Inuk), the accepted term in Canada.

2. MacDougall (2000) estimated this number based on previous research and statistics. However, with the information from the current documentation project, I think this estimate is too high. I was able to get information from inhabitants of nine communities about deaf people in their community. If the percentage given by MacDougall is accurate, there would have to be 75 deaf people in those communities (based on the Canadian Census 2006). However, the actual number of deaf people identified by the inhabitants of those communities totalled 20.

3. Manually Coded English is a sign system, using ASL signs but English word order, and adding signs encoding English grammatical items. It is outside the scope of this research to determine whether the deaf Inuit who do not use IUR, use ASL or MCE specifically, or maybe a version in between these two. As most lexical signs in MCE come from ASL, the non-native sign language is referred to as ASL in this chapter.

4. It should be clear that the language used between the researcher and PU is not ASL, as the researcher only has a basic knowledge of ASL. The communication took place in a form of Manually Coded English, in which PU was very comfortable, and the researcher adapted to him as much as possible. See note 3 also.

5. But c.f. Pullum (1991) who argues that English, like other languages, also has quite a number of words for 'snow' and 'ice'.

6. A Deaf community is a community of Deaf people that share a culture and a sign language.

7. Please note that this describes which languages were used in these schools at the time the deaf Inuit adults attended. No claims are made about the schools' languages nowadays.

8. I thank Terry Janzen (University of Manitoba) for pointing out that HOME in ASL conversation might lose the movement, too. The citation form of the sign has a movement though, which is retained by PU who, as described in the text, is the model of the ASL signs for other IUR signers. 


\section{References}

Antzakas, K. \& B. Woll

2002 Head movements and negation in Greek Sign Language. In: I. Wachsmuth and T. Sowa (eds.) Gesture and Sign Language in Human-Computer Interaction, 193-196. Berlin: Springer.

Boyes Braem, P. \& R. Sutton-Spence (eds.)

2001 The hands are the head of the mouth: The mouth as articulator in sign languages, Hamburg: Signum.

Brennan, M.

2001 Making borrowings work in British Sign Language. In: D. Brentari (ed.), 49-86.

Brentari, D. (ed.)

2001 Foreign vocabulary in sign languages. A cross-linguistic investigation of word formation, Mahwah: Lawrence Erlbaum.

Brentari, D. \& C.A. Padden

2001 Native and foreign vocabulary in American Sign Language: A lexicon with multiple origins. In: D. Brentari (ed.), 87-120.

Condon, R.

1983 Inuit behavior and seasonal change in the Canadian Arctic. Ann Arbor: UMI Research Press.

Davis, J.E.

2010 Hand Talk. Sign Language among American Indian Nations, Cambridge : Cambridge University Press.

De Vos, C.

2011 Kata Kolok color terms and the emergence of lexical signs in rural signing communities. The Senses \& Society 6:1. 68-76.

Dorais, L.-J.

1990 Inuit uqausiqatigitt. Inuit languages and dialects. Iqaluit: Arctic College.

Dorais, L.-J.

2010 The Language of the Inuit. Syntax, semantics, and society in the Arctic, McGill-Queen's University Press.

Fedden, S.

2011 A Grammar of Mian, Berlin: De Gruyter Mouton 
Harper, K.

2003 Inuit writing systems in Nunavut: Issues and challenges. In F. Trudel (ed.), Building Capacity in Arctic societies: Dynamics and shifting perspectives. Proceedings of the $2^{\text {nd }}$ IPSSAS Seminar. Québec City: CIÉRA, 91-100.

Huhn, E.S.

2004 Knowledge systems. In: T. Biolsi (ed.), A Companion to the Anthropology of American Indians, 133-153. Oxford: Blackwell,

Johns, A.

2007 Restricting noun incorporation: root movement. Natural Language and Linguistic Theory 25.3. 535-576.

Kaplan, L.D.

2005 Inuit snow terms: how many and what does it mean? In F. Trudel (ed.), Building Capacity in Arctic Societies: Dynamics and shifting perspectives. Proceedings of the $2^{\text {nd }}$ IPSSAS Seminar, Québec City: CIÉRA 249-255.

Kulchyski, P.

2006 Six gestures. In: P. Stern and L. Stevenson (eds.) Critical Inuit Studies. An anthology of contemporary Arctic ethnography, 155167. Lincoln: University of Nebraska Press.

Kusters, W.

2003 Linguistic Complexity. PhD dissertation, Universiteit Leiden. Utrecht: LOT Dissertation Series No. 77.

Lupyan, G. \& R. Dale

$2010 \quad$ Language structure is partly determined by social structure, PLoS ONE, 5:1. 1-10.

MacDougall, J.

2000 Access to justice for deaf persons in Nunavut: Focus on signed languages. Report for the Research and Statistics Division, Dept. of Justice, Canada.

Marsaja, I G.

2008 Desa Kolok. A deaf village and its sign language in Bali, Indonesia. Nijmegen: Ishara Press.

Martin, L.

1986 "Eskimo words for snow." A case study in the genesis and decay of an anthropological example. American Anthropologist 88. 41842 
McGrath, R.

1984 Canadian Inuit Literature: The development of a tradition. Ottawa: National Museums of Canada.

Meier, R. P.

2002 'Why different, why the same? Explaining effects and non-effects of modality upon linguistic structure in sign and speech.' In: R.P. Meier, K.A. Cormier and D.G. Quito-Pozos (eds.), Modality in Signed and Spoken Languages, 1-25. Cambridge: Cambridge University Press.

Miller, C.

2001 The adaptation of loan words in Quebec Sign Language: Multiple sources, multiple processes. In: D. Brentari (ed.), 139-174.

Nonaka, A.

2004 The forgotten endangered languages: Lessons on the importance of remembering from Thailand's Ban Khor Sign Language, Language in Society 33. 737-767.

Nonaka, A.

2007 Emergence of an indigenous sign language and a speech/sign community in Ban Khor, Thailand. Unpublished PhD Dissertation, University of California Los Angeles.

Nyst, V.

2007 A Descriptive analysis of Adamorobe Sign Language (Ghana). $\mathrm{PhD}$ dissertation, Universiteit van Amsterdam. Utrecht: LOT Dissertation Series No. 151

Nyst, V.

2012 Shared sign languages. In: R. Pfau, M. Steinbach \& B. Woll (eds.), Sign Languages, 552-574. Berlin: Mouton de Gruyter.

Olsthoorn, T.

2010 Die Erkundungsreisen der Herrnhuter Missionare nach Labrador (1752-1770). Kommunikation mit Menschen einer nichtschriftlichen Kultur, Hildesheim: Olms

Pullum, G.

1991 The great Eskimo vocabulary hoax and other irreverent essays on the study of language. Chicago: University of Chicago Press

Rasing, W.C.E.

in prep. The transformation and transfer or Inuit knowledge. Notes on isumaqsajuq, ilisajuq and qaujumajatuqangit.

Reece, L.

1970 Grammar of the Wailbri language of Central Australia, Oceania Linguistic Monograph 13, University of Sydney 
Schermer, G.M.

1990 In Search of a Language. Influences from spoken Dutch on Sign Languages of the Netherlands, Delft: Eburon

Sutton-Spence, R. \& B. Woll

1999 The Linguistics of British Sign Language, Cambridge: Cambridge University Press

Van der Kooij, E.

2002 Phonological Categories in Sign Language of the Netherlands. The role of phonetic implementation and iconicity. $\mathrm{PhD}$ Dissertation, Universiteit Leiden. Utrecht: LOT Dissertation Series No. 55

Wachowich, N.

1999 Saqiyuq. Stories from the lives of three Inuit Women. Montréal: McGill-Queen's University Press

Washabaugh, W.

1980 The Organization and Use of Providence Island Sign Language, Sign Language Studies 26. 65-92

Washabaugh, W.

1986 Five Fingers for Survival, Ann Arbor: Karoma

Whorf, B.L.

1940 Science and linguistics. Technology Review (MIT) 42:6. 229-31 \& 247-248. Reprinted in J.B. Carroll (ed.).

Whorf, B.L.

1956 Language, Thought, and Reality: Selected writings of Benjamin Lee Whorf, Cambridge: MIT Press, 207-219

Zeshan, U.

2000 Sign Language in Indo-Pakistan. A description of a signed language, Amsterdam: John Benjamins Publishing Co.

Zeshan, U.

2003 Aspects of Türk İşaret Dili (Turkish Sign Language). Sign Language and Linguistics 6:1. 43-75

Zeshan, U.

2004 Hands, head, and face: Negative constructions in sign languages.

Linguistic Typology 8. 1-58. 


\title{
An exploration in the domain of time: From Yucatec Maya time gestures to Yucatec Maya Sign Language time signs
}

\author{
Olivier Le Guen
}

\section{Introduction}

Time is generally considered an abstract conceptual domain and although it can be divided on the basis of more or less complex calendar calculations, all human cultural groups have some ways of expressing temporal relations in language, which can be spoken or signed. This chapter discusses how linguistic divisions for time have been constructed in an emerging language, Yucatec Maya Sign Language (hereafter YMSL) used by individuals in the Yucatán Peninsula in Mexico. Because of its sociolinguistic setting, YMSL is actively in contact not only with spoken Yucatec Maya but also with Yucatec Maya co-speech gestures that provide signers with manual input for the construction of the time domain. I will argue that Yucatec Maya speakers produce gestures with time reference that have been taken up and adapted in YMSL by signers.

Languages have diverse strategies for the linguistic expression of time (see Klein, 2010 for a comprehensive review). This chapter focuses on the deictic and sequential expression of time in Yucatec Maya speech and gesture and in YMSL. In the deictic expression of time, the time of an event is localised with respect to the time of the production of speech (e.g. I'll leave tomorrow), while in the sequential expression of time, events are often related to each other independently of the time of speech production (e.g. I will leave after the party, August follows July).

Many languages use spatial metaphors to talk about time and Fauconnier and Turner (2008, p. 55), for instance, assert that "Time as Space is a deep metaphor for all human beings. It is common across cultures, psychologically real, productive and profoundly entrenched in thought and language." However, more recent studies in non-Western settings suggest that this mapping may not be universal (Sinha, Sinha, Zinken, \& Sampaio, 2011). 
I will show that spoken Yucatec Maya has relatively few linguistic tools to talk about sequential time (compared to English or French for instance) and that sequential events are expressed with a rolling gesture that implies cyclicity rather than linearization of events or orientation of time flow. In other words, there is no metaphorical time line in the Yucatec Maya gestural space, and this specific form of gesturing about time is taken up and adapted in YMSL. This contrasts with speakers of languages such as French (Calbris, 1990), who use a time line to organise sequences of events as left-past to right-future, or signers of many sign languages, who productively use a front-future and back-past strategy (Kendon, 1993; Meir \& Sandler, 2008; Valli, Lucas, Mulrooney, \& Villanueva, 2000, inter alia).

The chapter is structured as follows: Section 1 gives an overview of the sociocultural context shared by Yucatec Maya and YMSL. Section 2 summarises the main linguistic strategies for the expression of time in spoken Yucatec Maya and section 3 examines the co-speech gestures that relate to time. In section 4, I discuss the expression of time in YMSL and how time gestures have been adapted as signs in YMSL. Section 5 summarises the main issues raised in the chapter.

\section{The sociocultural context}

Yucatec Maya Sign Language (or YMSL) is defined here as a signed language that develops in a Yucatec Maya speech community. This implies that YMSL signers share a cultural background with Yucatec Maya speakers and that spoken Yucatec Maya and YMSL are two languages actively in contact. This chapter focuses on a particular village, Chican, where many deaf and hearing individuals have been developing a sign language also referred as Chican Sign Language. Johnson (1991) gives a comprehensive overview of the sociolinguistic situation of Chican in the late 1980's. Escobedo Delgado, this volume, provides an updated sociolinguistic sketch (see also Zeshan et al., this volume).

\subsection{The socio-linguistic context of spoken Yucatec Maya and YMSL in Chican}

Yucatec Maya is a language spoken in the Yucatán peninsula in Mexico and Northern Belize, with the number of speakers approximating 786,000 in 2010 (INEGI, 2010). The Yucatán peninsula is a flat terrain covered with semi-tropical forest. 
In Chican (or Chi' Kaan 'the snake's mouth' in Yucatec Maya), ${ }^{1}$ all women older than 60 are monolingual in Yucatec Maya and even if most men and members of the younger generation are bilingual, interactions in the village are still conducted in Yucatec Maya. Spanish is learnt at school and used only with non-Mayan interlocutors. However, many children are socialised in Spanish and now speak it within the household. Dramatic linguistic changes may be occurring in the next generations.

Many men in Chican practice subsistence corn farming, using a slash and burn type of agriculture. A number of families also have pigs, poultry, or even some cows. The basic staples cultivated in Chican, as in many other Mayan populations, are corn (prepared as tortilla), beans, and other cucurbitaceous vegetables (e.g. pumpkins, squashes).

In the last few years, different sources of income have emerged. Currently, many people from the village go to work in the city. Typically, men take jobs in the construction industry (albañiles) and women work as housekeepers. Two Chican deaf women work as housekeepers in Mérida, the state capital, and come back to the village regularly on the weekends, although not every week (LTP and MCC, see Table 1 below). Handicrafts, especially hammock weaving (wak'k'áan) have become a significant source of income for many deaf and hearing families in the village. An increasing tendency, at least among hearing men, is to go to the USA or Canada for temporary work.

The forest in this region is low and big trees and palms are rare. In the past, houses were made of wood; walls were built with thin branches of wood woven together (kolox che') covered with clay, and thatched roofs were made of palm leaves. But because such material is now hard to find or too expensive to import from other states of the Peninsula, the current tendency, often supported by governmental support, is to build houses with concrete blocks.

As far as its sociological composition, I would consider Chican a 'family village,' insofar as the village was founded by members of a single family, and most of the villagers are related to some extent by kinship. One crucial cue is given by the family surnames that, in Mexico, encompass both the father's and the mother's side. It is significant that most of the villagers, and many of the deaf in particular, bear identical first and last surnames (Collí Collí in this case), indicating that their father and mother are somehow related. This means that the deafness in Chican probably has a genetic origin. The fact that one deaf couple has two deaf children also provides additional support for the genetic hypothesis of deafness in this village.

The YMSL Chican community is, to this day, the largest deaf community identified in the Yucatec Peninsula. It comprises 17 signers. In Yucatec Maya, 
various terms are used to refer to deaf persons. The term kook means 'deaf' and refers more generally to people with any kind of hearing impairment. One term that specifically applies to 'deaf people' is toot but it is mostly used by elders. Due to the general influence of Spanish language and Mexican culture (in Chican, this influence also includes other non-Mayan nonMexican visitors) the loan term soordo-muudo 'deaf-mute' is also widely used. Generally, Yucatec Maya people recognise deaf as people 'having no (verbal) words/voice,' mina'an ut'an. Interestingly, to refer to how signers communicate or to cite a signed utterance, Yucatec Maya speakers use the verb e'es 'show' (as in ba'ax kuye'esik ... 'what (s)he is showing is...') and not the verb $a$ 'al 'say' used for the spoken language.

\subsection{Yucatec Maya attitudes towards deafness}

As a community, the Yucatec Maya are tolerant towards deafness. As noted by Johnson (1991), and in contrast with western settings where deaf communities arise, there is no discrimination against the deaf. A similar attitude is also noted by Branson et al. (2002), as well as in de Vos, this volume, for the village locally known as Desa Kolok in Bali. More generally, there is no standard in the Yucatec Maya ideology of a 'normal' or 'fully capacitated' human being. In the Yucatec Maya culture, everyone is considered different and each person is apprehended as a 'different word' (kaada máak yáanal mundo, see Hanks (1993, p. 221)). In general, Yucatec Mayas believe that God created children just the way they are and that they should be accepted that way. This fact was explicitly stated by parents of deaf children interviewed during fieldwork. In a sense, deafness is considered by the Yucatec Maya as a trait of the individual, as is temperament (some people are ts 'ilk, 'fierce' and, according to informants, "that's the way they are," beey umoodoo') or skin colour (a common girl nickname is $x$ 'Boxi 'the black' and the author's nickname is griingo 'the white foreigner'). The perception of deafness as a personal characteristic rather than a handicap contrasts with the perception of deaf people in non-indigenous Mexico who use the Spanish term: discapacitado 'disabled, handicapped.'

Sociologically, deaf individuals are fully integrated into Yucatec Maya society. As pointed out by Johnson (1991), deaf people work and marry just as hearing people do. As long as one participates and contributes productively to daily activities and chores, he or she is fully incorporated into the Maya social setting. As do their hearing counterparts, deaf women cook, make handicrafts and take care of children, and deaf men can work in the 
fields or engage in other manual work just as hearing men do. There is no restriction on deaf people in their choice of spouse and deaf people marry other deaf people, as well as hearing people. In Chican, two men are married to hearing women and there are two deaf couples, one with 2 children.

The only real sociological difference from hearing people lies in the education deaf individuals receive. As pointed out by Poy Solano (2011), deaf children are not literate because the Mexican educational system cannot integrate them as students, making them de facto monolingual in YMSL. In contrast, many inhabitants of Chican are trilingual to various degrees in Yucatec Maya, Spanish, and YMSL.

\subsection{Speech communities}

Typically, signers of YMSL are persons who are born deaf (see Escobedo Delgado, this volume, for data on the deafness ratio in Chican). In contrast with some deaf children who are raised in urban environments with highly restricted language input (see for instance Goldin-Meadow and Mylander (1984)), Yucatec Maya deaf children are not isolated interactionally and are surrounded by many individuals. A typical Yucatec Maya family is composed of at least three children (and often up to eight or ten). Linguistic interactions in a typical Yucatec household rely heavily on multimodal channels and especially on the gestural channel. This particular sociolinguistic situation is characterised by a high degree of use of 'quotable gestures' (Kendon, 1992), and thus provides a deaf child growing up in a Yucatec Maya environment the systematic input required to develop a signed language. That is, Yucatec Maya speakers produce a lot of gestures, and these gestures have, in their majority, a consistent form as well as retrievable and constant semantics (see Le Guen (2011a) for the case of space). One cue that supports the hypothesis of transfer from gestures to signs is that in domains that are not systematically encoded in Yucatec Maya gestures (e.g. colours) YMSL tends to be more idiosyncratic, among speakers as well as among variants.

One important feature to point out is that a deaf child (or adult) in a Yucatec Maya setting is never alone. Yucatec hearing people acquire YMSL through extensive interaction with the deaf (there is no formal teaching), but during this process they also participate in constructing the sign language on the basis of their extensive gestural repertoire and gestural habits. We can distinguish two types of hearing interlocutors, or co-signers, who are all bimodal bilinguals in spoken Yucatec Maya and YMSL: those who have a deaf person in their family and those who live in the same village but are 


\section{Olivier Le Guen}

not related (or very distantly related) to a deaf person. The family members who live with or close to a deaf person greatly stimulate the development of the signed language. Peers usually constitute everyday interlocutors and are themselves fluent in sign language. In contrast, the parents of deaf children are usually poor signers. This is due to the fact that among Yucatec Mayas, parents usually interact quite minimally and asymmetrically with their children, primarily sending them to do chores (Gaskins, 1999, 2006). The second type of interlocutors are other members of the village, whether distant family or unrelated, who display varying degrees of proficiency in the sign language. The speakers who have good metalinguistic awareness of the Yucatec Maya gestural repertoire can easily communicate with deaf signers at a basic level, even if they have never before been in the presence of deaf people. ${ }^{2}$ Having a good grasp of one's gestural repertoire is a first step to understanding YMSL.

Deaf people engage in chatting daily and are often accompanied by hearing people (Johnson 1991, pp. 468-469). It is mostly men who gather in the street, usually at night, while women, following a more general Mayan pattern, rarely leave the household except for short trips during the day (to go shopping or to grind corn) and for public events. The 'deaf-only' gatherings that happened in Chican were always triggered by external factors, such as meetings initiated by the government or by foreign researchers. ${ }^{3}$

\subsection{Interactional groups}

Defining generations of signers is a delicate issue since age alone is not a straightforward criterion. According to Kisch (this volume), groups of signers are better defined in terms of cohorts or interactional groups, i.e. speakers who have been socialised as a coherent speech community. Another important feature that frames communication in the Yucatec Maya cultural setting is the family network. Mayan people primarily interact with their family members, and proximity between households does not necessarily guarantee social interaction. Non-kin neighbours habitually do not engage in communicative exchanges (chatting, asking favours, etc.) unless they have no other choice. ${ }^{4}$ For instance, the neighbours who lived in front of a family with various deaf signers explained to the author that they do not know the sign language, for they never interacted with deaf people.

In Chican, I consider there to be six interactional groups of signers, overlapping to a great degree with settlements. The age range of deaf people is between 78 and 10 years of age, and some interactional groups comprise siblings/cousins or deaf parents with deaf children. The first generation is 
composed of only one signer, who is the oldest deaf person in the village (DnT, approximately 78 years old); the second generation is made up of 13 adults (from 19 to 57 years of age), and the third generation is comprised of 3 deaf children aged 10,13, and 14 years. Data collected during the summer of 2011 suggest some intergenerational differences. Preliminary results from tasks that were meant to elicit ditransitive constructions tend to show an evolution in the use of space among the members of the second generation, who are deaf children born to deaf parents. This is particularly interesting because similar processes of conventionalization have been described in other incipient sign languages; see for instance Senghas et al. (2001; 2004; 2002) on Nicaraguan Sign Language or Sandler et al. (2005) on Al-Sayyid Bedouin Sign Language.

Across the three generations described above, six interactional groups can be identified (summarised in Table 1). A family of four constitutes the first interactional group, two deaf parents and their two deaf children. Three other relatives of interactional group 1 (brothers and cousins) form interactional group 2. In interactional group 2, StCC is married to a hearing woman and they have two hearing children. GUC has two hearing parents and hearing siblings. Interactional groups 1 and 2 live almost in front of each other and interact regularly. Adults of interactional groups 1 and 2 have been the main informants of Johnson and have been in close contact with the cinematographer Hubert Smith. MCC, the older sister in interactional group 2, goes to work regularly in Mérida and has not been continuously present in the village. Interactional group 3 contains three deaf siblings and their various hearing family members. LTP also has been working for several years as a housekeeper in Mérida, for a wealthy family who decided to help her financially so that she could take lessons in Mexican Sign Language. As a result she shows some peculiarities in her signing with respect to other YMSL signers. However, she and other members of her family said that her Maya interlocutors did not like the borrowed MSL signs she was using at first and that she now shifts from MSL to YMSL whenever she comes back to Chican. The third member of interactional group 3, BTP, has a young hearing child. Interactional group 4 was originally composed of 3 deaf siblings and their hearing siblings and parents. However, in 2010 CCC died unexpectedly of a heart attack and a marriage is planned between LCC and ACC (from interactional group 5), which means that she will leave her home and go to live with (or nearby) the members of interactional group 5. Interactional group 5 is formed by 3 deaf siblings who live alone in the same house. Signers of interactional group 5 and interactional group 3 are related by kin, live close by and have regular interactions with each other. Interactional group 6 is formed 


\section{Olivier Le Guen}

by a deaf man married to a hearing wife with several children. Finally, interactional group 7 is formed by the oldest deaf person in the village and his hearing family members: his son and his son's wife and children.

Table 1. Interactional groups with deaf signers in Chican

\begin{tabular}{|c|c|c|c|c|}
\hline & & $\begin{array}{c}\text { age } \\
\text { (approx.) }\end{array}$ & gender & $\begin{array}{l}\text { hearing members in the } \\
\text { interactional group }\end{array}$ \\
\hline \multirow[t]{4}{*}{ group 1} & $\mathrm{JCC}$ & 55 & male & NO \\
\hline & $\mathrm{NCT}$ & 47 & female & \\
\hline & $\mathrm{MlCC}$ & 15 & female & \\
\hline & $\mathrm{CaCC}$ & 10 & male & \\
\hline \multirow[t]{3}{*}{ group 2} & $\mathrm{StCC}$ & 45 & male & YES \\
\hline & $\mathrm{MCC}$ & 57 & female & \\
\hline & GUC & 25 & male & \\
\hline \multirow[t]{3}{*}{ group 3} & BTP & 20 & female & YES \\
\hline & LTP & 18 & female & \\
\hline & RTP & 13 & male & \\
\hline \multirow[t]{3}{*}{ group 4} & LCC & 55 & female & YES \\
\hline & $\dagger \mathrm{CCC}$ & 42 & male & \\
\hline & VCC & 35 & male & \\
\hline \multirow[t]{3}{*}{ group 5} & $\mathrm{ACC}$ & 45 & male & NO \\
\hline & SICC & 50 & female & \\
\hline & $\mathrm{ECC}$ & 55 & female & \\
\hline group 6 & ICC & 45 & male & YES \\
\hline group 7 & DnT & 78 & male & YES \\
\hline
\end{tabular}

$\dagger$ : died in 2010

Importantly, there are two types of interactional groups, those composed of deaf people surrounded by hearing interlocutors such as parents, siblings, spouses, and children (interactional groups 2, 3, 4, 6 and 7) and the interactional groups constituted by deaf individuals only (interactional groups 1 and 5). Undoubtedly, the fact that interactional groups comprise either hearing and deaf people, or deaf people only gives different impulses to the sign language varieties in those interactional groups. It is important to under- 
line that YMSL is what I would call an 'opportunistic language' since its primary interest lies in the efficiency of communication and not so much in the systematization of the language. As a consequence, we notice in the lexicon some idiosyncrasies within interactional groups, acknowledged by the signers themselves, and even among individuals. For instance, although ECC lives with her two deaf siblings, she has created some signs of her own. However, in the domain of time, no significant variations were noted (besides lexical ones, such as the days of the week, see below).

In the following section I explore in more detail the conception of time in spoken Yucatec Maya, in co-speech gestures and in YMSL.

\section{Time in spoken Yucatec Maya}

Yucatec Maya lacks grammatical tense. In short, this means that its resources for relating two events that occur at different times from the moment of the utterance are highly constrained. For instance, example (1) in English would have to be expressed as (2) in Yucatec Maya. Note that example (2) can be a present, past or future event.

(1) Lila entered while Joe was speaking on the phone

(2) táan u-tsikbal ti' telefono Jo(e)-e' $k a$ ' h-hook Liila PROG 3A-talk FOC phone Joe-TD CONJ CP-enter Lila

'Joe is speaking on the phone and Lila enters'

In (2), only progressive and completive aspect is marked, which means that in the absence of other temporal information, the event could be occurring at the moment of utterance production. Secondly, the ordering of the events in Yucatec Maya should fit their chronological order in the utterance. The conjunction $k a^{\prime}(a h)$ is only a generic temporal connective and can be translated according to context as when, then or and. In (2), the conjunction could have been replaced by a full stop, changing the coordinate clause introduced by $k a$ ' $(a h)$ into a construction with two juxtaposed main clauses. The conjunction $k a$ ' $(a h)$ does not express any ordering relation; it only indicates that the time of the main clause is somehow related to the time of the coordinate clause. The order of events is then inferred from the order of the clauses on the basis of implicature. Because Yucatec Maya also lacks time connectors (e.g. before, after, while), the ordering of the events is crucial to the meaning of the sentence. 


\subsection{The expression of sequential time}

Bohnemeyer (2009) proposes that Yucatec Maya relies on temporal anaphora, which is "the contextual determination of topic times" in any given utterance. ${ }^{5}$ He shows that the ordering of aspectual operators is crucial to understanding sequences of events: Whereas completive aspect implies a new topic time, the use of imperfective or progressive aspect includes the sentence in the running time of the event described, until a new completive marker comes to 'reset' the running discourse time. Therefore, in order to express sequences, Yucatec Maya uses completive markers as "natural reference points", for instance the expression ken ts'o'ohke'/ka'ah ts'o'oke' 'when it will be/was done.' In order to convey the meaning of example (3), Yucatec Maya should make explicit the state of completion of each event, which is presented separately and ordered chronologically, as in (4). A more extensive discussion on time in Yucatec Maya grammar can be found in Bohnemeyer $(2003,2009)$ and Vapnarsky (1999). A discussion on time sequence and spatial metaphors in Yucatec Maya is also presented in Le Guen and Pool Balam (2012).

(3) wash your hands before and after eating

\begin{tabular}{|c|c|c|c|}
\hline ken ts'o'ok-ok & $a-p^{\prime} o^{\prime}-i k$ & $a-k^{\prime} a b-e^{\prime}$ & k-a-taal hanal \\
\hline CONJ finish-SUBJ & 2A-wash-TR.IC & 2A-hand-TD & HAB-2A-come eat \\
\hline ken ts'o'ok-ok & $k-a-b i n$ & $a-p^{\prime} o^{\prime}-i k$ & $a-k^{\prime} a b$ \\
\hline CONJ finish-SUBJ & HAB-2A-go & 2A-wash & 2A-hand \\
\hline
\end{tabular}

'when you're done washing your hands, you come eat, when it's done, you go wash your hands'

\subsection{The expression of deictic time}

If Yucatec Maya only has a limited set of linguistic strategies to express sequences of events, forms of expressing deictic time are abundant. Crucially, deictic time expression always considers the time of the production of the utterance. Yucatec uses a large number of adverbs and particles to express deictic time such as 'uch, 'distal past time', $k a$ 'achi', 'distal past time (within lifetime frame)', ho 'oloh, 'the day before', sáam(y-ak), 'recent past (within the day)', táant, 'immediate past in terms of minutes (within the day)', be'oora,'now', 
walak(-il-a'),'now/at the same time as now', ta'ayt(-ak), 'immediate future in terms of minutes (within the day)', mun-xáan-tal,'immediate future in terms of minutes, hours (within the day)', mun-(y)úuch-tal,'immediate future in terms of days', biin, 'remote, prophetic future.'

In addition, Yucatec Maya has a set of what I would refer to as 'indexical adverbs' that specifically refer to past and future days with respect to the time of the production of the utterance, such as óxyak, 'three days ago', ka'ahvyak, 'two days ago', ho'olyak, 'yesterday', o'nyahak, 'yesterday in the evening', behla('ak)e', 'today, nowadays', sáamal, 'tomorrow', ka'abeh ', 'in two days', óoxeh, 'in three days.'

Temporal adverbs can be used to set up a reference point in discourse to locate the time of the events, as in (5). Indexical adverbs on the other hand tend to take the syntactic slot of the aspect marker, as in (6). The implication is that indexical adverbs directly tie the narrated event to the time of utterance production.

(5) úuch-il-ak-e'

táan u-máan Hesukrìisto way yóok'ol kàab-e' AM-NOM-TEMP-TD PROG 3E-pass Jesus here on earth-TD

'Long ago, Jesus-Christ walked this Earth' [lit. 'In remote past, JesusChrist is walking here on the Earth']

(6)

$\begin{array}{ll}\text { óoxeh } & \text { in-bin } \\ \text { +3.days } & \text { 1A-go }\end{array}$

'I'll go in three days' [lit. 'three days from now, I go']

In sum, in Yucatec Maya, the expression of sequences of events is highly constrained: events should be ordered chronologically because the existing connectors express essentially the completion (or non-completion) of events. Importantly, in Yucatec Maya, each sentence bears its own aspect and events are considered separately. In Yucatec Maya however, it is possible to insert past events like flashbacks in discourse under specific conditions (see Bohnemeyer, 2003, pp. 155-156 for details). On the other hand, Yucatec Maya is able to express deictic time with precision and has an important set of adverb markers that can be used to place events with respect to the moment of the utterance production. 


\section{Time Gestures in Yucatec Maya}

In order to address the question of time gestures, spontaneous discourses (including one natural conversation in which the researcher was not present) were analysed. ${ }^{6}$ The data were collected among Yucatec Maya speakers of Kopchen and Chemax who are not acquainted with deaf people or YMSL signers. We looked specifically at gestures produced with time reference in a corpus of 4 different contexts that totalled 63 minutes. We concentrated on deictic adverbs that set a reference point in time (e.g. úuch 'a long time ago') and on indexical time adverbs (e.g. sáamal 'tomorrow'). Data and results are presented in Table 2 and Table 3.

Additionally, we also asked five speakers to produce some conventional gestures, among them some time gestures, and speakers produced the citation form for each gesture. That is, these gestures are usually well-formed and bigger than what we found in the spontaneous data, though the gesture shape is similar. We asked participants how they would gesture the following deictic time expressions: be'oora/behlae' 'now/these days,' sáamal 'tomorrow,' ho'olyak 'yesterday,' ts'uyúuchtal 'it was a long time ago,' yan uyúuchtal 'it will be in a long time,' sansáamal 'everyday,' kaada áanyo 'every year.'

Results from the analysis of spontaneous and elicited gestures show three main types of time gestures used among Yucatec Maya speakers. All three gesture types are mapped onto the spatial domain. 


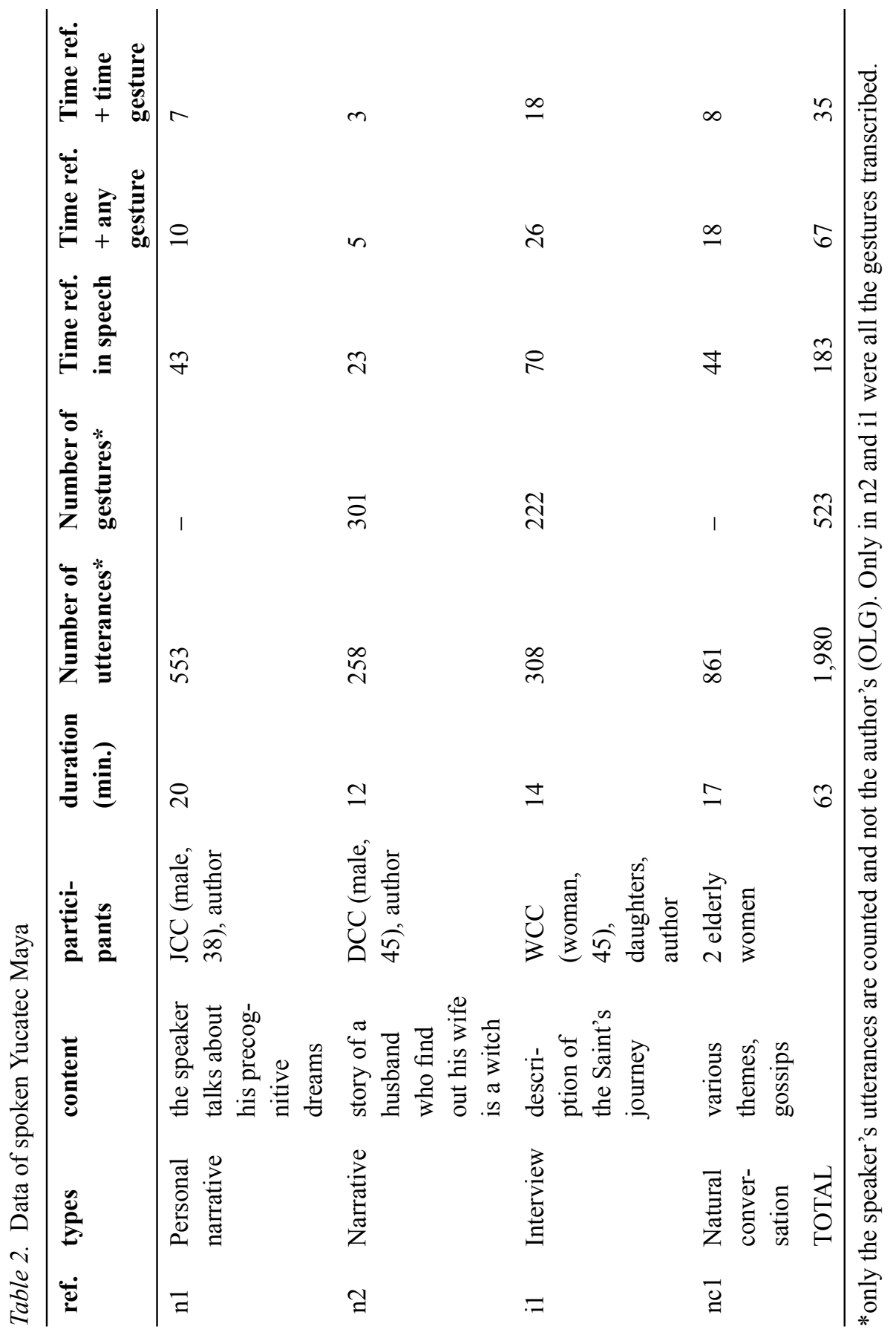


Table 3. Gesture types occurring with time adverbs and time reference

\begin{tabular}{lccccc}
\hline Gesture type & Here-now & Distant & Rolling & Pointing & Counting \\
\hline $\begin{array}{l}\text { Personal } \\
\text { narrative (n1) }\end{array}$ & 2 & - & 6 & - & - \\
Narrative (n2) & 1 & - & 1 & 1 & - \\
$\begin{array}{l}\text { Interview (i1) } \\
\text { Natural }\end{array}$ & - & - & 6 & 5 & 4 \\
$\begin{array}{l}\text { conversation } \\
\text { (nc1) }\end{array}$ & & 3 & 3 & 1 & 2 \\
TOTAL & $\mathbf{3}$ & $\mathbf{3}$ & $\mathbf{1 6}$ & $\mathbf{7}$ & $\mathbf{6}$ \\
\hline
\end{tabular}

\subsection{The here-now gesture}

The here-now gesture is used to refer to precise space (way-e' 'here') and metaphorically precise time (now). This gesture is widely used across cultures and languages and is not in any way specific to Yucatec Maya. This here-now gesture usually co-occurs with time references such as be'oora 'now' or te' semana he'ela' 'this week.' It is typically done with a finger pointing towards the feet of the speaker.

\subsection{The distant time and space gesture}

The distant time and space gesture is used to refer to distant space (very far and/or not known/uncertain) and metaphorically to ancient or future time. This type of gesture is primarily used for unknown space. Yucatec Maya speakers (and YMSL signers) use a geocentric Frame of Reference and tend to use all the gestural space that surrounds the speaker for spatial information. They always use direct pointing to refer to existing places, and not metaphorical pointing when the referent is too distant or if its location is unknown, as Westerners do, see McNeill (2003), McNeill et al. (1993). Thus, if a distant or remote referent lies behind them, they will point in that direction (see Le Guen, 2011b for a detailed explanation of gesture production in relation to frames of references). Basically, when Yucatec Mayas point to existing places the orientation of their gestures is always accurate with respect to the location of the place mentioned. This is not specific to Yucatec Maya, but also occurs in other spoken languages (cf. Haviland (1993, 2000), Levinson (2003) or 
Dasen and Mishra (2010), inter alia). Furthermore, Yucatec Mayas also use the space surrounding their bodies to locate a distant figure and a distant ground in virtual space with respect to their actual location, i.e. if the figure is north and the ground south, they will point to locate the figure to the north of their body and place the ground southward, usually south of their body (see Le Guen (2011a) for more details). Such use of the gesture space to express spatial information involves a continuum from very precise information indicated in the here-now gestural space, towards a more distant-remoteunknown information located upwards; the middle space is commonly used to point to existing locations, i.e. only for spatial reference. Remote space is localised on top of the head of the speaker, and this is where distant time is mapped. Interestingly, in Yucatec Maya gestures, both the past and the future are metaphorically mapped onto the same distant space gestural space: above the speaker's head, but never backwards. The distant time and space gesture usually occurs with time references such as úuch (ka'achi') 'very distant past', but also with yan uyúuchtal 'distant future' (see Figure 2 in Section 3.4). The lack of metaphorical timeline for temporal gestures that oppose 'not now' vs. remote time has not yet been documented in spoken languages but it is attested in sign languages (see section 5). This conflation of past and future also exists, unsurprisingly, in YMSL, as shown below.

\subsection{The rolling gesture}

The rolling gesture is used to refer to repetitive events and time unfolding. Elicitations conducted with several informants as well as results from nonverbal tasks (see Le Guen and Pool Balam, 2012) made clear that Yucatec Maya speakers do not conceive of time unfolding as a line, i.e. events are not organised along a metaphorical line in space (neither front-back, leftright or down-up). Yucatec Maya speakers, as the linguistics of time in their language would predict, conceive of events in terms of their completion and, to put it briefly, for Yucatec Mayas 'time does not go anywhere.' In order to represent an event's completion or more generally time unfolding, speakers use variations of the rolling gesture, allowing them to represent it visuo-spatially, as happening at one and the same point in space. Among Yucatec Mayas, the rolling gesture seems the only way to represent time unfolding. In the representation of sequences of events, one rolling gesture would represent one event and the next gesture the following event, etc., and this corresponds to the more general non-linear cyclic conception of time in this culture (see Le Guen and Pool Balam, 2012, for more detail). The rolling 
gesture occurs in spontaneous discourse with time references such as kàada áanyo 'every year' but also tusigyèente diya/ken sáaschahke' 'the next day.' The rolling gesture occurred 16 times out of the 35 total time gestures (46\%). This gesture is performed with one hand or one finger (10 gestures, $63 \%)$ or with both hands, one rotating around the other (6 gestures, 38\%). The rolling gesture is not, however, always performed as a full circle (i.e. a 360 degree movement, Figure 1a) but is also realised as a half circle (i.e. a 180 degree movement, Figure 1b). Often a flat hand or a finger is placed at the chest level, around which the dominant hand rotates, as presented in Figure $1 \mathrm{~b}$.
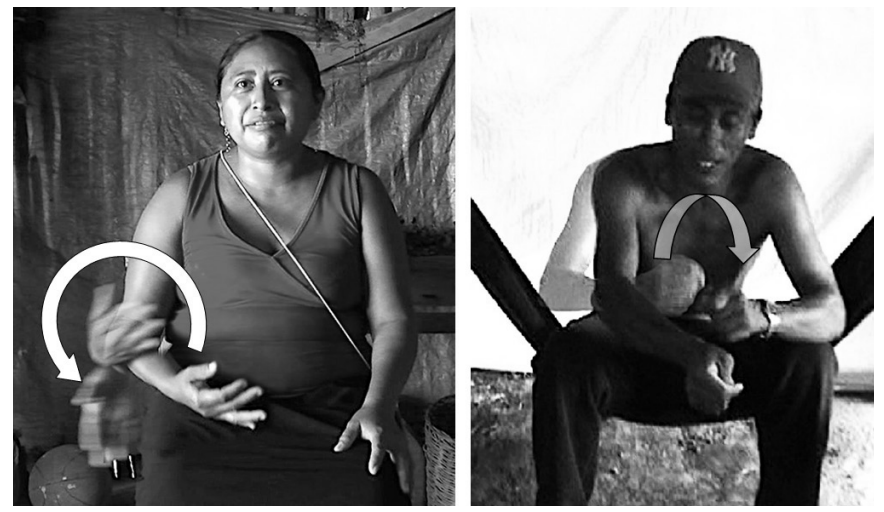

Figure 1. Example of rolling gesture (a) 360 degrees and (b) 180 degrees

\subsection{No distinction in gesture between past and future direction}

What is striking in the way that the Yucatec Maya gesture about time is the fact that they do not distinguish past and future. This contrasts with many spoken languages where speakers consistently use a metaphorical gestural time line (e.g. front-back) to make this opposition between past and future (Calbris, 1990; Casasanto \& Jasmin, in press; Cooperrider \& Núñez, 2009; de Jorio, 2000; Kendon, 1993; Núñez \& Sweetser, 2006, inter alia). The absence of a time line in the Yucatec Maya gestural space also coincides nicely with the way in which a succession of events is linguistically expressed, i.e. in terms of completion, with no directionality. Additionally, it also reflects the more general cyclic conception of time where events are thought to unfold and replace each other in the same place.

Data from elicitations and interviews shows that the distant time and space gesture forms used to express past and future are similar to each other, 
as shown in the following examples of participants gesturing ts' uyúuchtal '(it was) long ago' (Figure 2a) vs. yan uyúuchtal 'it will be in a long time' (Figure $2 b)$. Equally, when participants were asked to gesture sáamal 'tomorrow' vs. ho'olyak 'yesterday' they did not contrast the orientation of the gesture for past and future, as in Figure 2c,d; instead, they produced two rolling gestures (180 degrees) with a similar orientation for both past and future.
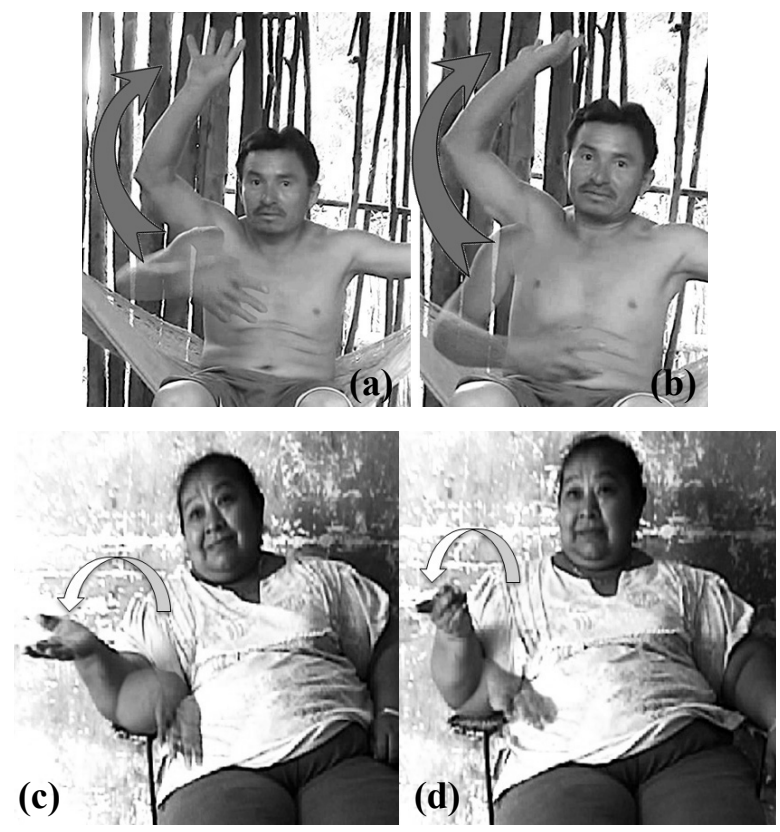

Figure 2. Gestures for (a) ts'uyúuchtal '(it was) long ago' and (b) yan uyúuchtal 'it will be in a long time' [IPM]; and gestures for (c) sáamal 'tomorrow' and (d) ho'olyak 'yesterday' [MBC]

In sum, for Yucatec Maya speakers, there is no metaphorical time line that expresses time unfolding. The here-now gesture used for precise time (and space) contrasts with distant/remote non-precise gesture for time (and space). It is also clear that in Yucatec Maya gestures for time, there is no opposition in directionality between past and future. In order to be able to gesture about time unfolding, the Yucatec Maya use the rolling gesture that, again, does not contrast past and future. Elicitations with informants show that they instead conceive of events as replacing each other in space (see Le Guen and Pool Balam 2012, for more details on this point). As a consequence, sequences of events have no linear organisation and no direction. The use of a geocentric frame of reference also constrains the use of the gestural space for time. 


\section{From time gesture to time sign}

In order to describe how time reference is accomplished in YMSL, I rely on two types of data: structured interviews designed to specifically elicit time signs and monologue narratives produced spontaneously by signers, addressed to me and to other signers. In this section, I show that many of the gestural strategies used among speakers of Yucatec Maya are taken up in YMSL and adapted to the specificities of this language. Like spoken Yucatec Maya, YMSL seems to lack grammatical tense (although it has lexical aspect). Additionally, as in Yucatec Maya, there is no metaphorical time line to organise sequences of events in space.

The reader will notice that not all the signs presented in this chapter are glossed in the same way. Some signs are specific to sign language grammars and are glossed accordingly (e.g. PRO-1 for first person). Others are glossed in Yucatec Maya and translated into English. The motivation for this choice lies in the fact that many signs have an equivalent in the Yucatec Maya gestural repertoire, from which they originate. In some cases, signs are calques from Yucatec Maya idiomatic expressions. Another fact that supports this choice is that translations of signs by bilinguals are usually in Yucatec Maya; when signs are translated into Spanish for non-Maya speakers, this is via the initial translation into Yucatec Maya. Not only are some of the bilingual informants more comfortable glossing signs in Yucatec Maya, but when conversing with a deaf person, they often start the first few sentences by signing and speaking Yucatec Maya at the same time. Finally, the lexical signs that have no direct equivalent in the Yucatec Maya gestural repertoire are directly glossed in English for convenience.

\subsection{Other forms of time keeping in Yucatec Maya and YMSL}

In this section I mainly describe forms of time keeping in YMSL that have their roots in gesture. More information on this matter can be found in Le Guen and Pool Balam (2012).

Although there is no metaphorical time line among Yucatec Mayas, they use the movement of the sun and of the moon to indicate the time of the day along a 'celestial time line' (cf. DeVos, forthcoming, for Kata Kolok). Speakers use metonymic pointing to indicate the position of the sun or the 
moon in the sky (in absentia) in order to refer to the time of the day. Thus, pointing straight up to indicate the position of the sun means midday and pointing 45 degrees east expresses a time around 10am. Crucially, these types of reference are limited to time of day and cannot be used to refer to past or future time in general.

Another common gestural strategy used by Yucatec Maya speakers to keep track of time is referring to the number, age and height of children. Speakers commonly refer to a particular event showing the height of a child (e.g. 'Last time you came, my first born was this tall [+ flat hand gesture].' This height gesture is used in YMSL as a human classifier (see Figure 12). ${ }^{7}$

Finally, in order to indicate sequences of events, Yucatec Maya speakers generally count on their fingers starting with the little finger (the smallest one meaning the smallest number) up to the thumb. For instance, in a discourse about the activities conducted during the Holy Days, the speaker refers to day 1 pointing to her little finger, to day 2 on the ring finger, etc. This strategy is also productive in YMSL (see Figure 13 below). These strategies are taken up and adapted in YMSL (see sections 4.3 and 4.4).

\subsection{Time units}

In YMSL there is no sign for 'day.' The use of a celestial time line, such as pointing to the sun to indicate the time of day is, surprisingly, not common among signers, although it is among speakers. As far as I am aware, the point to the moon is not used in YMSL. ${ }^{8}$ Still, signers are aware of the position of the sun and they indicate events like 'dawn' and 'dusk' instead of a particular hour. Conventional signs for (TUN) SÁASTAL '(BECOMING) CLEAR (i.e. DAWN)' and (TUN) E'HOCH'E'ENTAL '(BECOMING) DUSKY' have their roots in Yucatec Maya gestures. Examples in Figure 3 and Figure 4 show how the Yucatec Maya idiom for dawn tunp'il uyich k'iin 'the sun opens its eye' is gestured by a speaker from Kopchen not acquainted with deaf using opening hands (Figure 3 ) and how it is signed by a signer from Chican (Figure 4). In order to disambiguate the idiom "the sun opens its eye" from the action of "opening an eye" the signer first points to the east where the sun comes up. The YMSL sign seems to be a calque from the expression in Yucatec Maya language and gesture. The sign for dusk is done the opposite way by closing hands. 


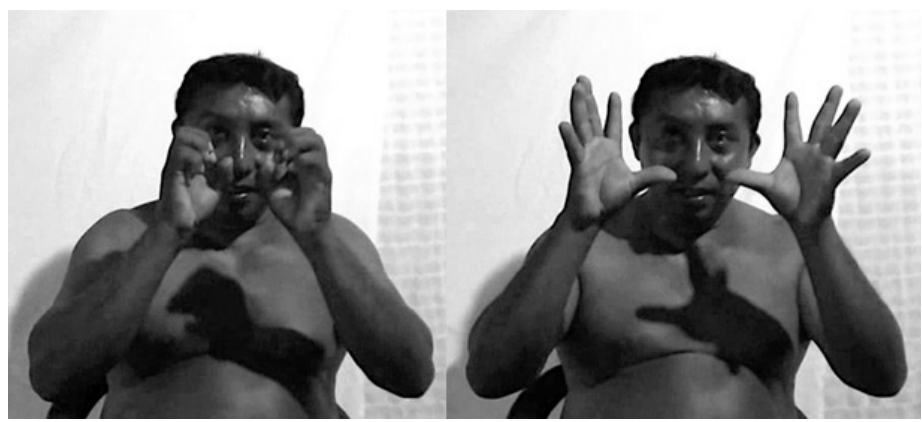

Figure 3. Speaker from Kopchen gesturing tunsáastal, tunp'il uyich $k$ 'iin 'it's becoming clear (dawn), the sun opens its eye' [DCC]

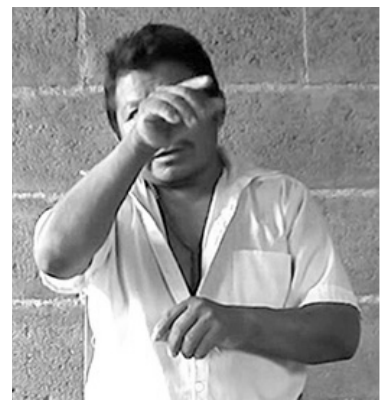

EAST

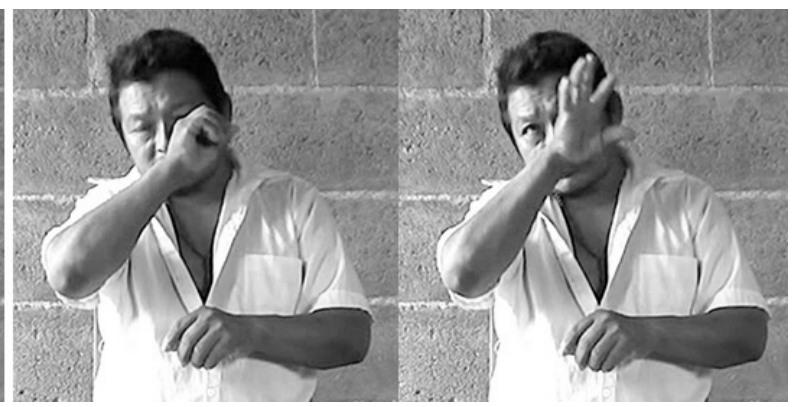

(TUN)P’IL UYICH (K'IIN) 'OPEN EYE (SUN)'

Figure 4. Signer from Chican signing DAWN [StCC]

Signers from Chican have invented signs for each day of the week and some signs for the months. The signs for the days are presented in Figure 5 by a woman bilingual in spoken Yucatec and YMSL, who is the sister and niece of various deaf signers of interactional groups 1 and 2. The sign for SUNDAY is done with both hands: thumbs up and index fingers extended in order to iconically represent a rifle. This is also the sign for RIFLE and HUNT, both $t s^{\prime}$ on in Yucatec Maya, because Sunday is typically the day when people go to hunt collectively (called $p$ 'uh in Yucatec Maya). The sign for MONDAY reproduces the salute to the flag, the Spanish saludo a la bandera, done by students on Monday when entering school. Note that in interactional groups 4 and 5, for instance, signers do not use the sign for MONDAY, but the sign for SUNDAY plus one rolling gesture, which is the sign PASSt(ime) (see Section 4.3), i.e.: SUNDAY+1. The sign for TUESDAY is MONDAY +1, and, in some interactional groups, it is SUNDAY+2 (i.e. two rolling signs). WEDNESDAY is done either with two fingers next to the head opening and 
closing, or with the hand above or on top of the head and shaking with all the finger tips pointing upward. According to some informants, this sign refers to a children's program that is broadcast on Wednesdays. However, in some interactional groups, WEDNESDAY is signed with the index finger oriented towards one's arm to iconically represent a syringe or more broadly 'getting a shot,' indexing the fact that government doctors and nurses come on Wednesdays to provide consultations and medicines. The sign for THURSDAY is WEDNESDAY (either sign) +1 . The sign for FRIDAY is done with an open hand waving from one side to the other. This sign represents the movement of the fanning of the matador's cape and refers to the televised corrida (bullfight) on Friday. Other bilingual informants have also identified the same sign as meaning HAMMOCK, for Friday is the day when the hammocks woven by people in Chican are picked up by foreign contractors. SATURDAY is done with a fist punching the jaw and represents the act of boxing, indexing the airing of boxing on Saturday. It is important to recall that at less than 70 years old, YMSL is a young language that evolved with modern media, including television, a visual input par excellence.

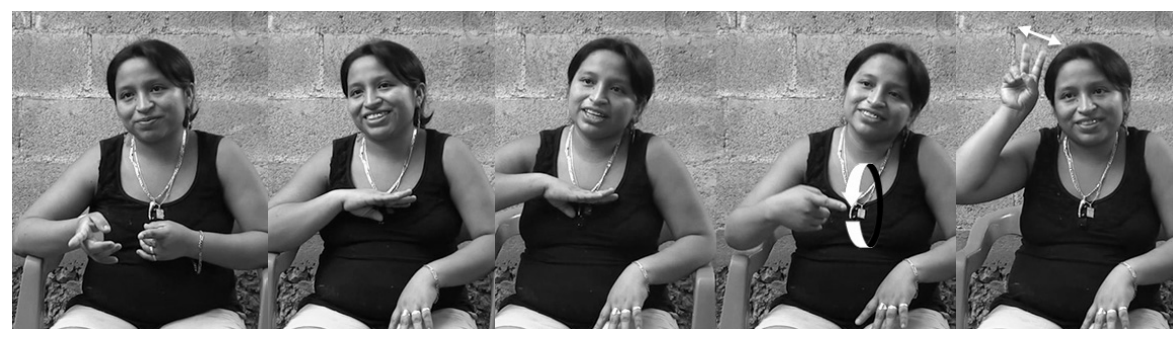

SUNDAY

MONDAY

TUESDAY (MONDAY +1) WEDNESDAY

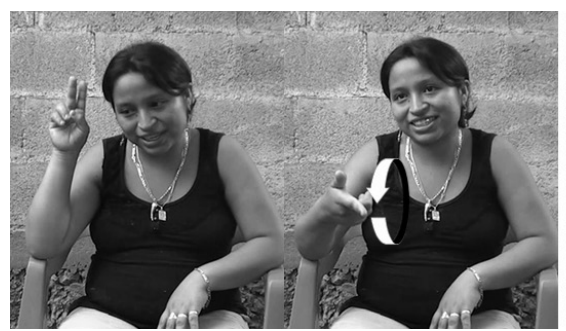

THURSDAY (WEDNESDAY +1)

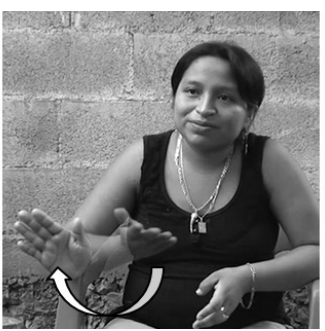

FRIDAY

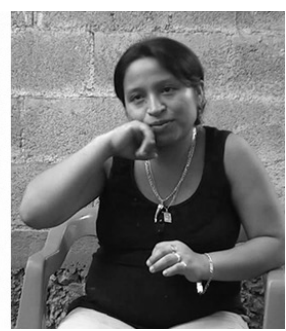

SATURDAY

Figure 5. The signs for days of the week in Chican [PUC]

Signs for names of months were only mentioned by bilinguals of interactional groups 1 and 2 and do not seem to be in use in everyday conversation. Signs for the days also vary among interactional groups and some signs seem to be known by everyone while others are not. However, this is 
not a problem for communication since signers always have the resource of adding the rolling sign, similar to the rolling gesture of the speakers, to add one or several day(s) to a sign for a day of the week. As shown in Figure 5, in Chican TUESDAY and THURSDAY are compounded as MONDAY +1 and WEDNESDAY +1 respectively. All signs of the days are iconic-indexical, at least in their original state, meaning that they iconically represent an event or part of an event and index the day that this event occurs. However, when deaf individuals and their co-signers were asked about the origins of signs, most of them could only retrieve with confidence SUNDAY, MONDAY and sometimes SATURDAY. This indicates that signs tend to lose their iconic meaning when they become conventionalised symbols (see for instance Keller, 1998).

Additionally, signers can also make use of numbers and days of the week to refer to deictic time (i.e. date is calculated from the moment of the utterance). For instance, a signer from Chican explains that she will get married in 7 weeks, using the names of the days and numbers to refer to a future event: PRO-1 7 SATURDAY 7 'I (will get married) in 7 Saturdays (from now).'

\subsection{Temporal and indexical adverbs}

Like spoken Yucatec Maya, YMSL has a number of ways to refer to deictic time, i.e. reference with respect to the moment of utterance production. Time adverbs are used, like in Yucatec Maya, to define a reference point in time. YMSL distinguishes between temporal adverbs NOW vs. REMOTE TIME (past or future) and indexical time deictics such as TOMORROW or IN 2 DAYS.

Time deictics in YMSL oppose present and non-present. The signs are formally and (to some extent) semantically equivalent to the spontaneous gestures described in section 3.1, the "here-now" gesture pointing at the speaker's feet, and the "distant time" gesture for remote past or future, performed over the speaker's head. I gloss the corresponding signs in YMSL as 'HERE-NOW' and as ÚUCH '(a) LONG TIME.' ${ }^{9}$ Figure 6a presents the sign for NOW / TODAY that can also mean 'here' (see section 5.1 on disambiguation). Figure $6 \mathrm{~b}$ presents the sign for ÚUCH 'LONG TIME' here produced to refer to past time. Figure $6 \mathrm{c}$ shows an idiomatic expression calqued from Yucatec Maya, ya'ab ubin 'in a long time in the future' (lit. 'a lot is to go') reproduced almost literally in sign. It is composed of the sign YA'AB 'A LOT' (done the same way in Yucatec Maya co-speech gesture but 
here with an additional intensifier expressed via a facial cue) and the ÚUCH sign. Interestingly, the mapping to space is equivalent since arguably, the distant time/space sign ÚUCH resembles the gesture typically produced with the verb bin 'go.' 10

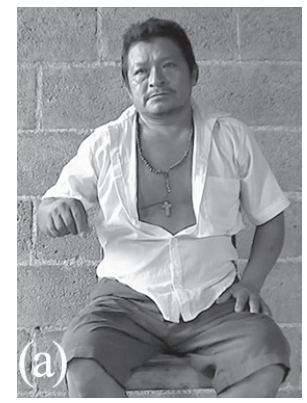

BE'OORA/BEHLA'AKE' NOW(ADAYS)/TODAY

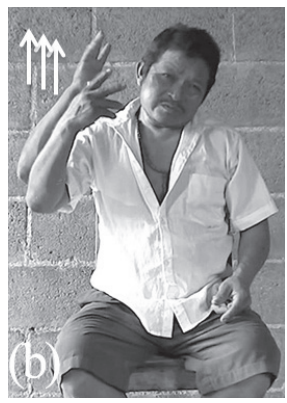

ÚUCH

LONG TIME

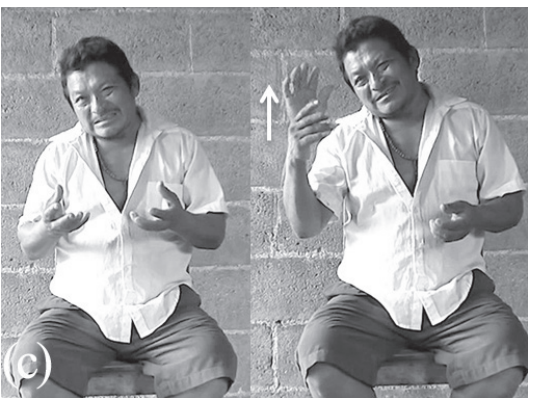

ÚUCH

IN A LONG TIME (future)

Figure 6. Time deictics in YMSL [StCC]

The rolling gesture has also been adapted in YMSL. Since in Yucatec Maya the rolling sign is glossed as bey umáan k'iin, lit. 'like time is passing' (from the verb máan 'pass by, move') but meaning something closer to the English 'time unfolding, time evolution', I shall gloss the sign PASSt(ime). Just as the rolling gesture described in section 3.3, the PASSt sign is produced either as a 180 degree semi-circle (Figure $7 \mathrm{a}$ ) or as a 360 degree rotation. It can mean either TOMORROW or YESTERDAY depending on the context. Some variation exists among signers and a specific sign for YESTERDAY, also sometimes used for past times, is occasionally produced as PASSt rotated backward, as in Figure 7b. However, note that in the elicited form presented in Figure 7b the signer first uses the sign TS'OK 'FINISH' (see below) in order to specify that he is talking about something that ended - that is, in the past - which contrasts with the sign he previously made for TOMORROW in Figure 7a. The use of the TS'OK sign is one strategy used to disambiguate future from past times (see section 4.4), as is the use of the sign PASSt produced with a backward rotation, although the latter does not yet seem to be systematised in the language and is rare in spontaneous discourse. As in ASL for instance (Liddell, 2003, p. 20), the integration of numbers in deictic time reference is productive in YMSL. In Figure 7c, the signers produced the number 2 and the PASSt sign to refer to KA'ABEH 'IN 2 DAYS.' 


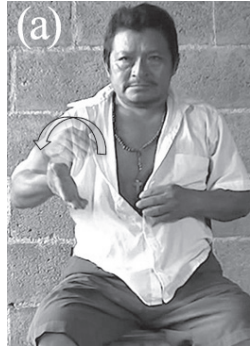

SÁAMAL/ HO'OLYAK

'TOMORROW/

YESTERDAY'

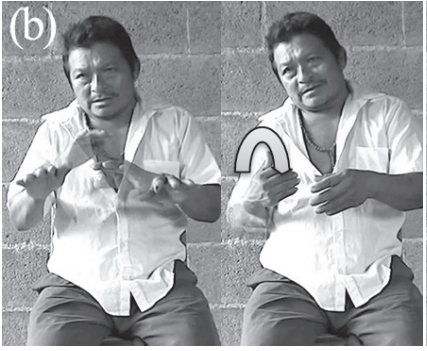

HO'OLYAK

'YESTERDAY'

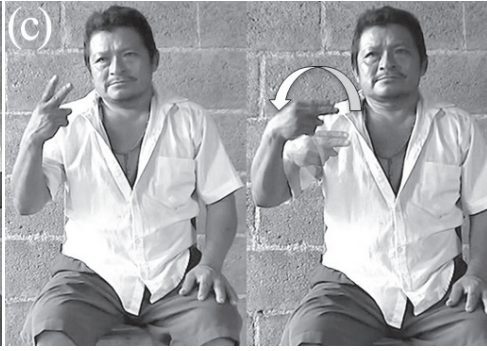

KA'ABEH

'IN 2 DAYS'

Figure 7. Indexical time signs in YMSL $\quad[\mathrm{StCC}]$

The sign EVERYDAY / A LONG TIME is done with the PASSt sign repeated several times. In this case, the sign can also be intensified either by slowing down the movement and/or making multiple circles and complemented with a non-manual facial marker ('pain face'). In Figure 8, a signer mentions that she is going to work tomorrow. The meaning of the PASSt sign as 'tomorrow' in this particular context relies on the fact that it is produced after the sign for HERE-NOW. Additionally, the reference to the work activity P'O' 'WASH' is a synecdoche for all the types of work she does (see also Figure 13 in Section 4.4), and the distant place (in this case Mérida, where the pointing is directed) reinforces the contextual interpretation of the sign as MONDAY, the day she is going back to work in the city. Note that the PASSt is done with both hands, one being stable at the chest, around which the other rotates 180 degrees, the same way it is performed in Yucatec Maya spontaneous gesture (Figure $1 b$ in section 3.3).

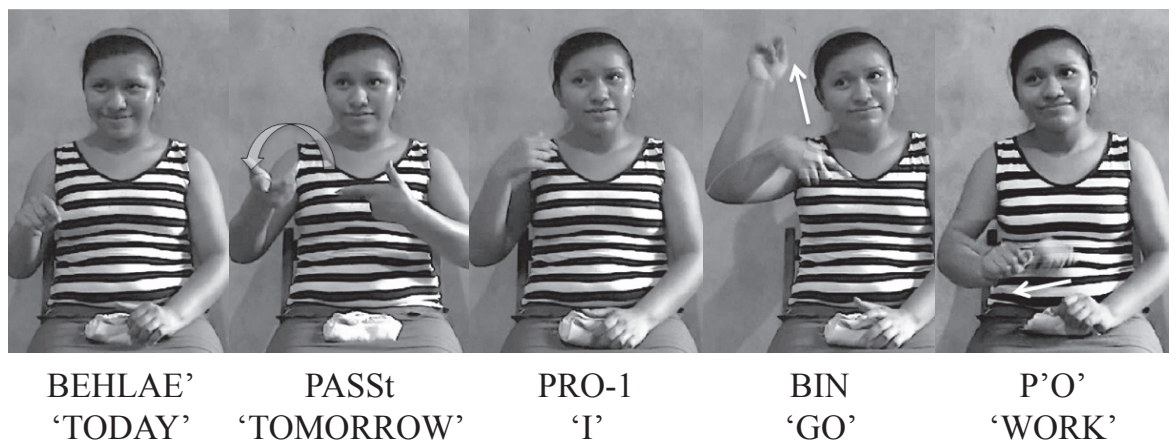

Figure 8. 'Tomorrow, I'll go to work (in Mérida)' [LTP] 
The sign PASSt can also be used as a deictic marker meaning 'several days from now,' as in Figure 9 where the signer explains that they will have a meal organised by a researcher (E.) with all the deaf in the village. In this case, the signer (GUC) does not give any precise time. The iterative production of PASSt (done twice, Figure 9c) conveys the meaning of 'several days' (from now). As a matter of fact, the same sign could also mean 'in 2 days from now,' but the prosody and the shared background knowledge that the meal will take place in a few more days is enough to disambiguate the meaning of the sign in this utterance. As mentioned above, if the speaker had wanted to be more precise about the date, he could have used a numeral along with the PASSt sign, as in Figure 7c.

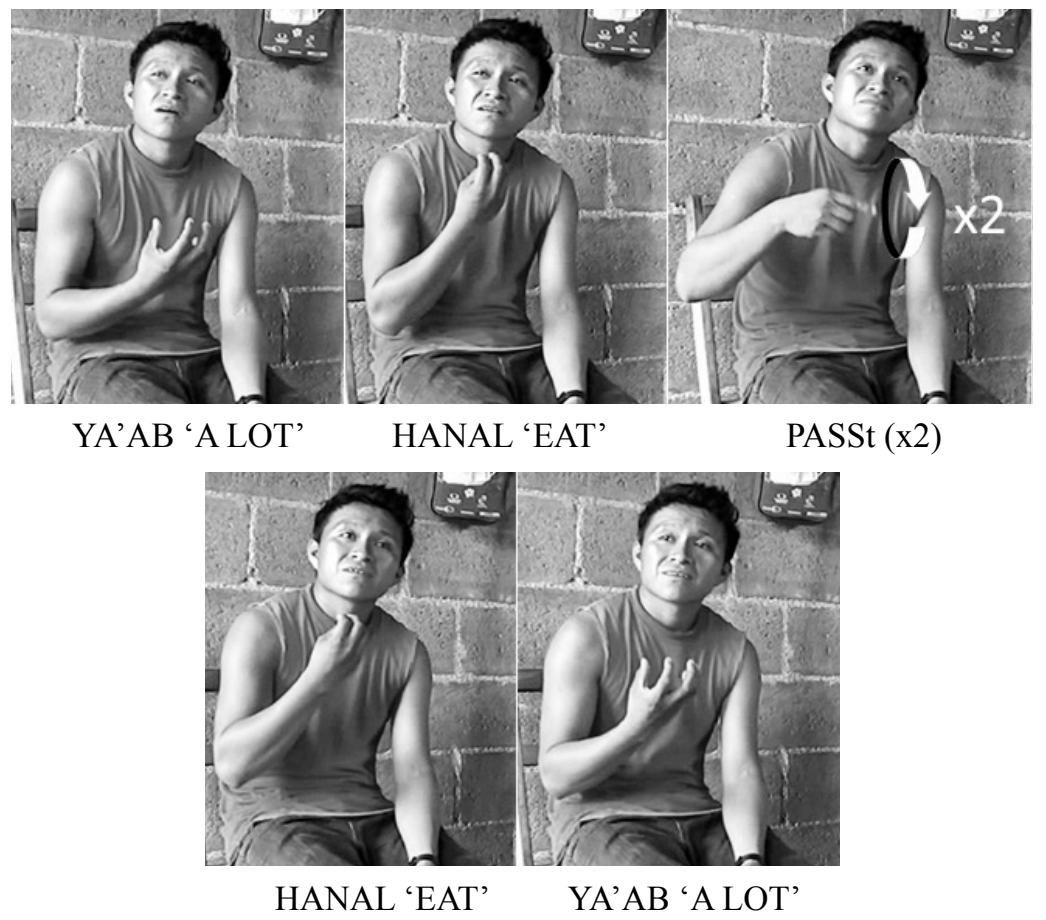

Figure 9. 'We will get together to eat in a few days, we'll eat together' [GUC]

An example used with 7 and PASSt to represent ' 7 weeks' is presented below in Figure 10. In this case, the inference that the event described will take place in seven weeks and not in seven days or months is based on previous shared knowledge. 


\subsection{Other signs used for time}

TS'OK sign could be glossed as 'FINISH.' The sign originates from the Yucatec Maya gesture TS'OK, which co-occurs with the completive marker $t s^{\prime}(o k)$ or other verbal references to completive events. Use of the YMSL sign resembles the completive aspect $t s^{\prime}(o k)$ of spoken Yucatec Maya, and therefore I shall gloss it as TS'OK. Note that in many sign languages, signs such as FINISH or WILL are used as aspect markers (see for instance Fischer and Gough (1999) or Valli et al. (2000) for ASL).

The gesture TS'OK is often produced at the end of Yucatec Maya narratives, usually with the verbal expression $t i$ ' $t s^{\prime} o$ 'oki' 'there it ends.' The gesture and the sign are performed in the same way: both flat hands cross each other at the centre of the body and move outwards towards the left and right. The gesture and the sign can also be performed with one hand. In YMSL, we note a similar use of the sign TS'OK at the end of narratives. But the use of the TS'OK sign in YMSL is much broader than the gesture use and seems to have evolved to act as a discourse maker, equivalent to a full stop in written language. It is very frequent at the end of utterances or chunks of discourses (see $\mathrm{TS}^{\prime} \mathrm{OK}_{2}$ in Figure 10 for instance). A similar description of completive aspect in Kata Kolok can also be found in de Vos (this volume).

The sign TS'OK seems to also be used as a marker of completive aspect, for instance before or after any given time sign. In Figure 10, a signer is talking about his future wedding. Since he is not married yet, we can safely assume that the events he describes are located in the future. In Figure 10, $\mathrm{TS}^{\prime} \mathrm{OK}{ }_{1}$ is performed after the PASSt sign is done twice and implies a new temporal reference 'when some time has passed' (in this case 7 weeks). The sign $\mathrm{TS}^{\prime} \mathrm{OK}_{2}$ on the other hand marks the end of the utterance. We note that the second TS'OK is larger than the first. Additionally, TS' $\mathrm{OK}_{1}$ is not prosodically detached from the surrounding signs, while for $\mathrm{TS}^{\prime} \mathrm{OK}_{2}$ the signer makes a short pause (1s) after the preparation phase of the sign (i.e. when the arms are crossed) and before the stroke (extended arms). 

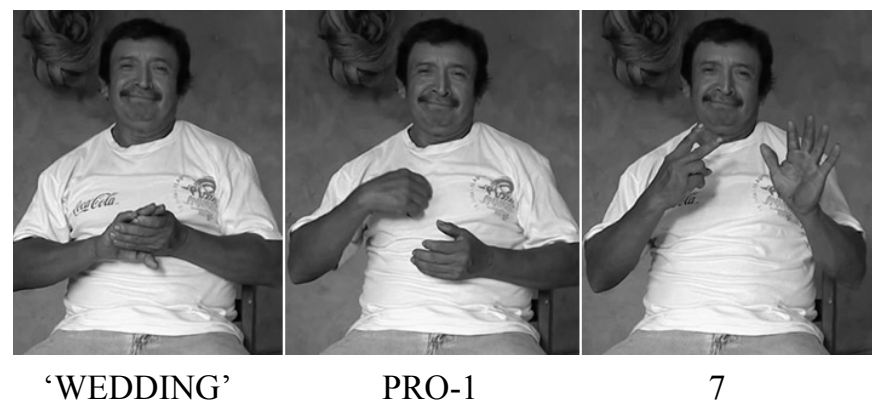

7

'I'

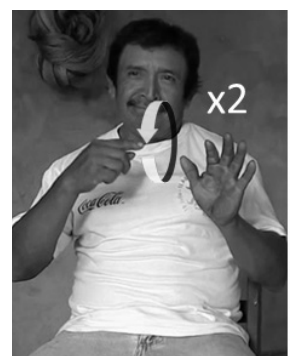

PASSt (x2)

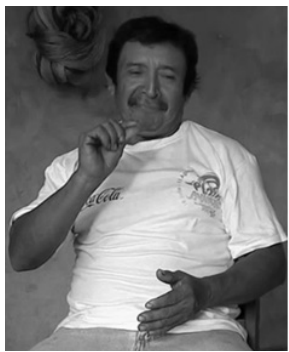

HUMP'ÍT

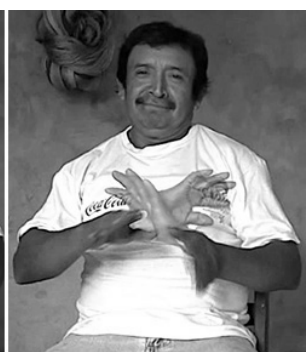

$\mathrm{TS}^{\prime} \mathrm{OK}_{1}$

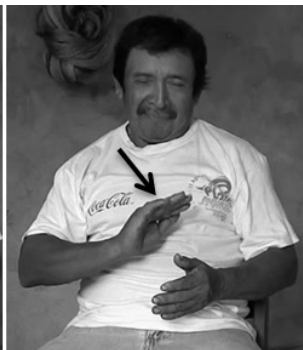

PA'ATIKI' 'WAIT'

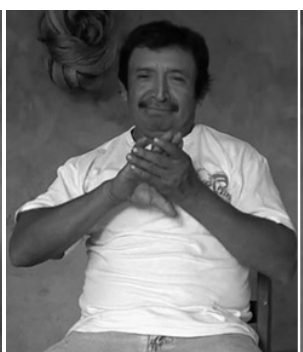

'WEDDING'

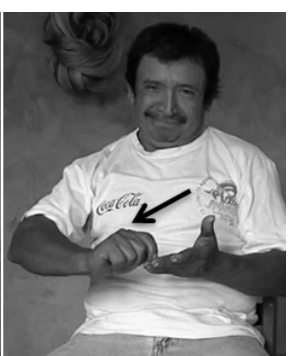

SIGN

'A LITTLE'

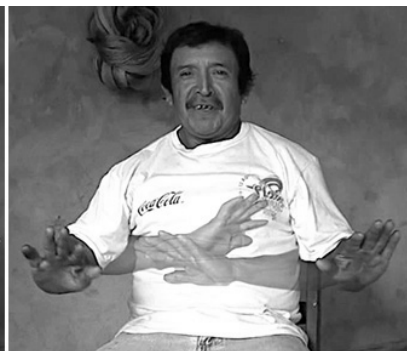

$\mathrm{TS}^{\prime} \mathrm{OK}_{2}$

Figure 10. 'I will get married in 7 weeks. It is a civil wedding (we'll sign the papers). Soon now (= in a little bit)!' [ACC]

Another use of TS'OK, also somewhat similar to Yucatec Maya, is to talk about sequences. In this sense, this use of TS'OK in YMSL is parallel to the conjunction $\mathrm{ka}^{\prime}$ 'ts'ohke'/ken ts'o'okok 'when it was/will be done' also used to describe sequences of events in spoken Yucatec Maya (see example 4 above). In the following example (Figure 11), a young signer describes the kind of work she does in the city. In order to separate each event, she uses the sign TS'OK that could be translated in English as 'and' or only as a comma. 


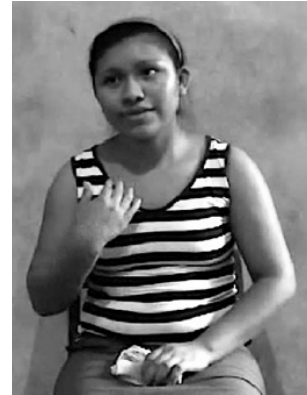

PRO-1 'I'

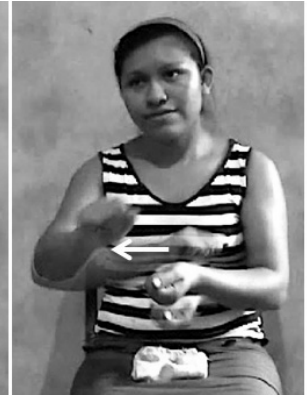

P'O' 'WASH'

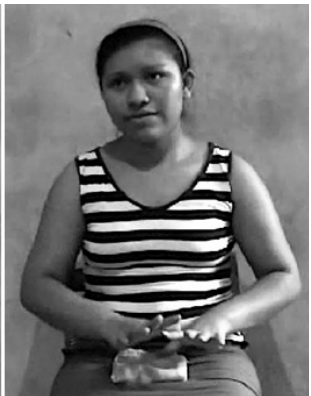

TS'OK

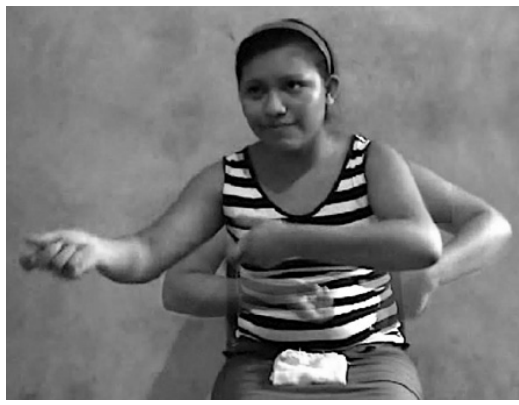

MOP

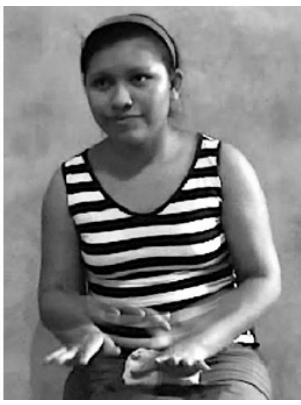

TS'OK

Figure 11. 'I wash (clothes) and I mop, and ...' [LTP]

Another sign also used for temporal reference is the sign HUMP'IIT 'A LITTLE.' This sign has a direct equivalent in Yucatec Maya gesture and, as in YMSL, can also refer to quantity of objects or quantity of time. It is done using the thumb and the index finger close to one another as if showing the size of something small. The sign is presented in Figure 10 (still 3 of the second line).

The sign PA'ATIKI' 'WAIT (FOR IT)' can also be used to refer to time. Among the Yucatec Mayas, this gesture is performed with an open flat hand that moves forward one or more times. PA'ATIKI' is a holophrastic gesture (i.e. a gesture that can replace a verbal utterance) meaning that it can be used with or without speech, and has some illocutionary or performative force, as in the case of 'come here' or 'go' (see Poggi cited in Kendon 1992). Holophrastic gestures occur frequently in Yucatec Maya conversation. Often, holophrastic gestures replace speech in a preliminary non-verbal stage of conversation among Yucatec Mayas. For instance, it is not uncommon for two speakers at some distance to communicate 'what's going on?', 'where are you going?', etc., using, e.g., a gesture with open hands moving outwards that functions as a question marker (used also as such in YMSL) and a point in the direction that one is going. In YMSL, although PA'ATIKI' can have 
illocutionary force, the sign is also used for temporal reference meaning 'wait for some time'; the two uses are differentiated by their syntactic context. When used for time reference, PA'ATIKI' seems to mark periods or intervals of time, though without precision. In some cases, it is used to refer to a period of time between two future events, as in Figure 10 where it follows the HUMP'IIT 'A LITTLE' sign. It is the sequential arrangement of the two signs that gives them their temporal meaning. In Figure 12, a signer relates how some people get robbed by government administrators, who keep for themselves the money they should distribute. In the following extract, the signer is talking about the case of his sister, who is single and does not get paid, although everyone else does, for the money comes from a Mexican national program. In the extract, the sign PA'ATIKI' is followed by the sign PASSt and implies that some time passes between the two events that precede and follow.

Finally, YMSL also uses buoys to make reference to time or sequences of events, a strategy also found in ASL (Liddell 2003, pp. 223ff). Figure 13 presents an example of the use of buoys in YMSL. In this example the signer summarises all the tasks she does as a housekeeper in Mérida. In order to describe the sequences of events (i.e. the different tasks) she uses buoys. Interestingly, buoys are used in the same way in YMSL and among Yucatec Maya speakers: counting from the little finger up to the thumb and again on the other hand (consider for instance the use of 6 in Figure 13 still 4 , second line). This manner of counting using buoys in YMSL contrasts with ASL, where signers use the index finger to stand as 1 and count up to the little finger (4) and, if necessary, add to thumb for FIVE-LIST buoys (Liddell 2003, p. 228). Note also the use of TS'OK 'FINISH' in Figure 13 to demarcate the two first events; TS'OK is not used afterward since it is redundant with the use of buoys. 


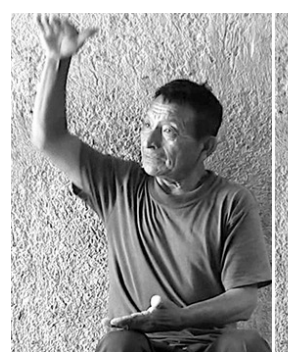

CLAS.H 'SISTER'
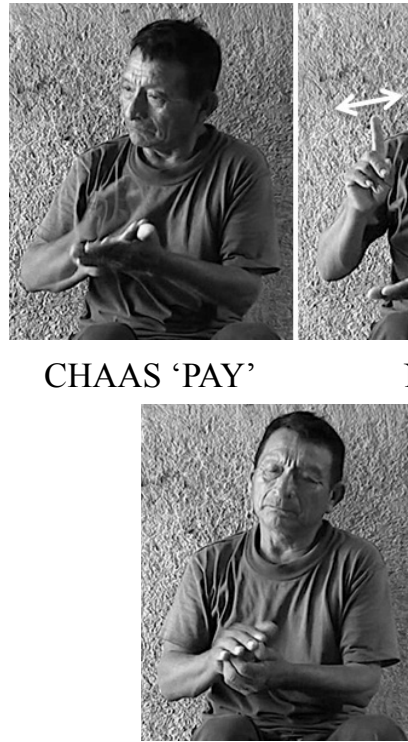

FOLD

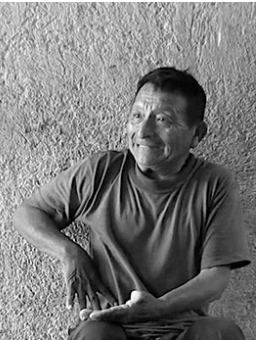

SIGN NAME 'MCC'

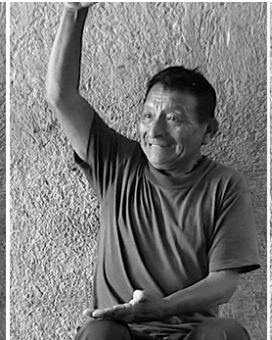

CLAS.H 'SISTER'

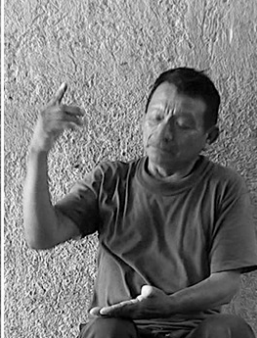

1 'ON ITS OWN'

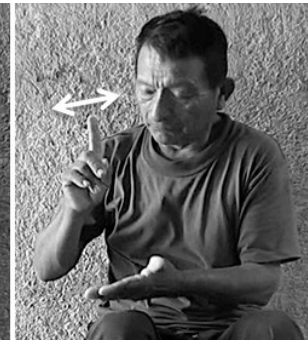

NEG.

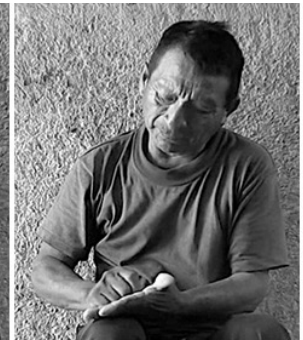

WRITE

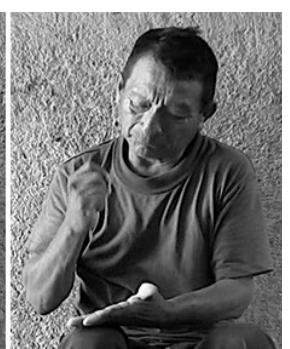

STAMP

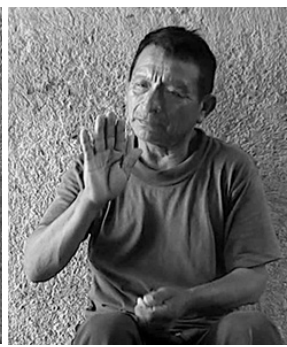

PA'ATIKI'

'WAIT FOR IT'

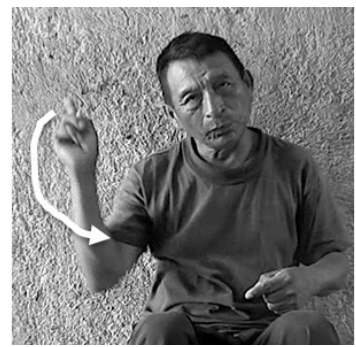

COME HERE

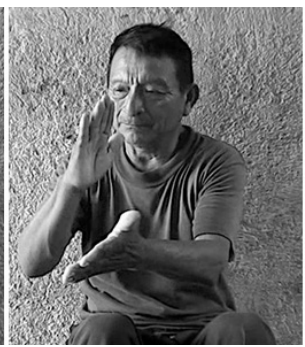

CHAAS 'PAY'

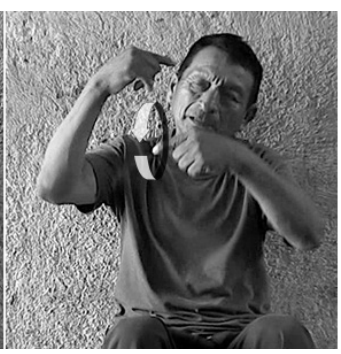

PASSt

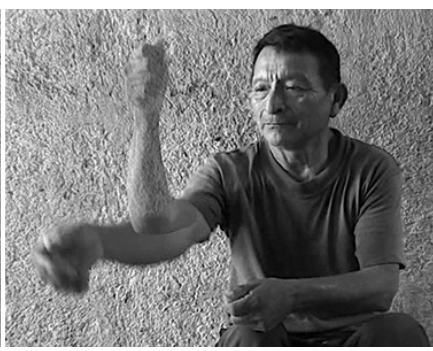

BO'OTIK 'PAY.trans.distrib.'

Figure 12. 'My sister MCC, she's on her own (not married), (but) she doesn't get paid, she (goes to) do all the paperwork, time passes and when (the government person) comes (in the village) he pays everyone' [JCC] 

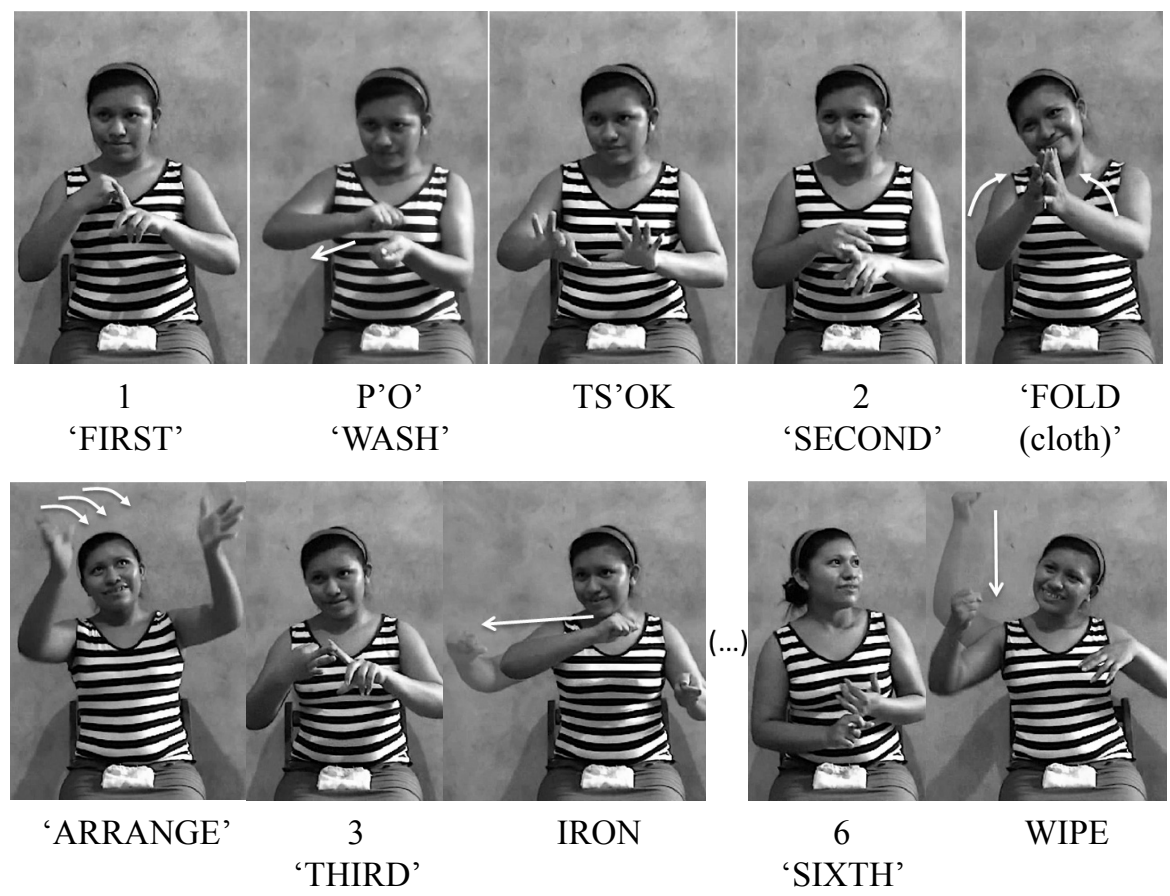

Figure 13. 'One thing I do is (hand-)wash (clothes). That's it (= that's one thing). Second, I fold (the clothes and) stack them. Third, I iron (the clothes) (...). Six, I clean the windows' [LTP]

\section{Discussion}

An examination of the conception of time in speech and gesture in Yucatec Maya and in YMSL shows that both are quite similar in the way they linguistically conceptualise deictic and sequential time. However, even if we can argue that Yucatec Maya and YMSL are two languages in contact, YMSL is an independent language that has developed specific strategies to talk about time, given its expression in only the manual modality in contrast with Yucatec Maya, which makes use of both verbal and manual channels. In this section, I discuss in more detail the linguistic evolution from Yucatec Maya gestures to YMSL signs in the domain of time.

\subsection{Disambiguation of time adverbs}

In the absence of grammatical tense, Yucatec Maya as well as YMSL use temporal adverbs to express discourse time and aspect (see de Vos, this 
volume for discussion of a similar phenomenon in Kata Kolok). Despite the mapping of space onto time and the lack of opposition between past and future in gesture, Yucatec Maya speakers can always use words to clarify or disambiguate when gesturing about time or space, past or present. However, when YMSL signers use the time and space gestures, they face two problems: first, disambiguating space from time, and second, in temporal reference, disambiguating past from future. Two main strategies emerge from the data for disambiguation in YMSL. The first happens at the discourse level and mainly relies on previous shared background knowledge about the event described (as exemplified in Figure 9 and Figure 10). At the utterance level, various forms of disambiguation are possible. One is compositionality. What I mean by compositionality is that two signs are produced contiguously and are not semantically independent from each other. That is, for some signs the spatial or temporal meaning is determined by the following sign. This is the case in Figure 7, Figure 8, Figure 10 and Figure 12. A second strategy relies on the utterance elements or surrounding context that allows for inference about space or time and past or future. For instance, in Figure 8 the signer says she is going to work and since the recording was made on Sunday we can infer that she means Monday. Finally, we note a tendency among certain bilingual signers and in particularly ambiguous contexts, to perform the PASSt backward (i.e. with a reversed rotation) to represent the past. This iconic solution allows disambiguation and is particularly suited to expressing time contrasts in the gestural modality.

\subsection{Evolution of co-speech gesture into sign}

It is striking that a large number of signs in YMSL originate in Yucatec Maya co-speech gestures (Shuman, 1980). This means that the Yucatec Maya gestural repertoire is not only vast but that many gestures belong to the category of 'quotable gestures' (Kendon, 1992) having a stable and systematic form and meaning. As a matter of fact, when speakers are asked to gesture concepts of time (as well as more lexical gestures, e.g. YA'AB 'many'), the data show a consistency in the gesture forms produced that suggests easy recall and recognition on the part of Yucatec Maya speakers. The fact that the two languages share a lot of gestural forms is one of the reasons why Yucatec Maya speakers acquire YMSL with relative ease.

Additionally, we also notice some calques (i.e. word-to-word translations) from Yucatec to the target language YMSL. This is unsurprising given that most of the signers are in fact bilingual in spoken Yucatec Maya and 
YMSL. The expression tunp 'il uyich k'iin 'the sun opens its eye' (Figure 4) is based on a cultural conception of the sun is an animate being having an eye, more precisely, a divinity referred to as Yum K' $i i n$ 'Lord Sun.' Such use in YMSL suggests cultural transmission and calibration between hearing and deaf people.

When gestures are adapted as signs, they undergo some important modifications. Besides the obvious changes in syntactic position, gestures become significantly more reduced when transformed into signs, and points of articulation may be modified. Compare, for instance, the citation form for UUCH in co-speech gesture and sign (Figure 2 vs. Figure 6). Phonological reduction is expected for sign language in order to facilitate language production; Zeshan (2003) considers this process of grammaticalization from gesture to sign under the category of 'loss of phonetic substance'.

YMSL has inherited from Yucatec Maya the absence of a metaphorical time line and, to some extent, the non-directionality of time flow. This reminds us that sign languages are not independent from the surrounding linguistic and cultural context in which they emerge. For instance, French, English (British and US) and Italian all show the use of a metaphorical time line used for deictic time in which the speaker's body represents the here and now, the future lies ahead, and the past is to the speaker's back. Not coincidentally, French, American, British and Italian sign languages also show a similar time line (see Kendon (1993), Valli et al. (2000) for ASL). However, de Vos (forthcoming) shows for Kata Kolok that signers do not use any metaphorical time line but only oppose present vs. non-present time. Kendon (1993) indicates that in Warlpiri, a secondary sign language used by aboriginal communities of Australia during mourning, the signs for future are not spatially distinct from those that refer to the past and that no movement (i.e. directionality) is involved. Unfortunately, these authors do not mention if the surrounding communities of speakers share the same absence of time lines in their co-speech gestures.

The data discussed in this chapter suggest that, like other sign languages, YMSL is constrained by the pre-existing linguistic/gestural material available among speakers. Imagine that signers of YMSL were to invent a time line similar to Israeli Sign Language (Meir \& Sandler 2008, pp. 101ff), using the space behind the speaker for the past and the space in front for the future, this would go against Yucatec Maya speakers' intuition about time flow, and would, in the end, seriously compromise interactions between deaf individuals and hearing bilinguals.

We also notice similar or parallel strategies to keep track of time between Yucatec Maya and YMSL. For instance, the use of numerals for deictic 
time reference is present in both Yucatec Maya (e.g. ka'a-beh 'in 2 days' is literally '2-paths', óox-yak '3 days ago', lit. 3-past.marker ) and in YMSL. However, Yucatec Maya has only lexicalised words for three days in the past and three days in the future and then jumps to eight days for a week cycle (for instance *ho'beeh 'in five days' does not exist in Yucatec Maya). YMSL signers in contrast use numeral incorporation productively. We also notice that YMSL as well as Yucatec Maya make use of buoys in order to refer to series and sequential time in the same way, starting from the little finger and going up to the thumb.

There are some arguments to suggest that absence of grammatical tense in YMSL is not inherited from Yucatec Maya. Pfau and Steinbach (2006) suggest that the grammaticalization of temporal concepts in language starts from lexical elements (nouns or verbs), then evolves to functional elements (adverbs) and eventually develops into affixation (i.e. tense or agreement). In sign languages, grammatical tense is rare, even if the surrounding spoken language has tense. For instance, although Hebrew and standard Arabic have grammatical tense, Israeli Sign Language does not (Meir \& Sandler, 2008, p. 89). ISL, like many sign languages, has a number of adverbial forms for time and several aspects (see also de Vos (forthcoming) for Kata Kolok). Tense as an inflectional category on verbs seems to be problematic for sign languages, especially tense inflection of verbs (but see Fridman-Mintz (2010) on Mexican SL). Movements in space are often already exploited to inflect verbs for pronominal reference, and additional changes in the form of the sign would increase difficulty in sign recognition. Several spoken languages around the world also lack to various degrees grammatical tense (see Bohnemeyer (2009) for references). The fact that both Yucatec Maya and YMSL rely on an identical system without grammatical tense makes it easier for bilinguals to express temporal relationships. In my own experience as a native speaker of French and speaker of other Indo-European languages, expressing sequences of time in Yucatec Maya represents a tremendous mental exercise, since using only temporal anaphora is not an intuitive strategy for speakers of languages with grammatical tense.

This exploration in the domain of time gesture and time signs shows that Yucatec Maya and YMSL are two languages in contact and that there are important transfers from Yucatec Maya to the emerging YMSL, facilitated by the vast repertoire of systematic co-speech quotable gestures of Yucatec Maya. We notice a similar conception of time and parallel forms to express deictic and sequential time in both languages.

Another conclusion that we can draw from the examination of the domain of time in Yucatec Maya and in YMSL is that village sign languages 
are not independent of their surrounding sociolinguistic context. It is not uncommon in sign language research to reject or simply ignore the importance of the gestures used in the surrounding speaking community as input for the construction of a sign language. Various researchers have however pointed out the limitation of such a stance (Fusellier-Souza, 2004; Marsaja, 2008; Russo \& Volterra, 2005, inter alia). Considering US English co-speech gestures, McNeill (1992) argues that these are mainly improvised with speech and hence have no stable form or meaning, unlike signs in sign languages. Such lack of systematicity in co-speech gestures is not without consequences in the development of non-institutional sign languages. For instance, GoldinMeadow and Mylander (1984) emphasise the poor input from American parents in the development of Home Sign systems in the US.

In this respect the Yucatec Maya setting contrasts drastically with the US setting. Yucatec Maya speakers gesture a lot (see Table 2) and accompany their speech with a substantial number of quotable gestures that have a stable form and meaning. As shown in this chapter, the importance of quotable gestures as an input for YMSL is visible in a semantic domain like time, but also in many lexical entries of YMSL, some shown throughout this paper. Crucially, the lack of systematicity between speakers or variants in certain semantic domains of YMSL arises in domains that are not 'gestured' in Yucatec Maya. For instance, colour terms vary dramatically between the two variants of YMSL in Chican and Nohkop because Yucatec Maya uses only spoken lexical items for this domain and no gestures. As a consequence, signers have to invent signs from scratch. In the time domain, we also notice some variation in sub-domains such as the names of the days. Yucatec Maya uses a verbal lexicon with loan words from Spanish for the names of the days of the week. Not surprisingly, this is where variations arise in YMSL: the Nohkop variant has only one sign for this sub-domain, the one for SUNDAY: K'OP (the sign is glossed after a conventional way of knocking someone's head with the fist). The sign, as many signs for days in Chican, has been invented with reference to a TV show for children aired on Sunday. In this show, one kid usually knocks his acolyte on the head. Calculation of the following/preceding days is done in reference to Sunday with the addition of the PASSt sign, as in Chican. In Chican, individual variations also exist among signers for the names of the days (see section 4.1), but not for the deictic time markers that are similar to or adapted from Yucatec Maya co-speech gestures. In sum, the specificities of the Yucatec Maya setting (notably the presence of an important quotable gesture repertoire but also the Maya attitude towards deafness) mean that deaf persons born in this setting are much better off in terms of communication and social integration than 


\section{Olivier Le Guen}

in most western contexts (where deaf people need to be taught conventional sign language within an institution, usually separate from other institutional settings where hearing people communicate).

Finally, the absence of a time line to order events sequentially and of directionality of time flow is original with respect to many spoken and sign languages described. Evans and Levinson (2009) point out the impoverished exploration of the possibility space for linguistic evolution in the linguistics research field, especially because of the focus on Indo-European languages. Hence, descriptions of new emergent non-western sign languages are crucial for sign language typology in particular, but also for language typology in general.

\section{Notes}

1. The reason for this name lies, according to some villagers, in that the village was constructed on a prehispanic setting and a big snake's head was found there. An alternative explanation for the name, also given by the villagers, is that it would originate from chi' ikam 'jicama' for the setting of the village would have been a place of abundance of this plant. Note that the village has changed name and is previously known as Nohya or, in Yucatec Maya, noh ya' '(the) big Chicozapote.' Note that official village names get reduced according to the phonology of Spanish and hence get modified in writing.

2. Several informants who have minimal interaction with deaf individuals have pointed out this fact. Also, my own experience as a fluent speaker of Yucatec Maya and that of my colleague, Lorena Pool Balam, a native speaker of Yucatec Maya, supports this notion.

3. During our stays in Chican with my colleague Lorena Pool Balam, we never attempted to gather deaf signers and have only visited deaf signers in their home.

4. Note that most of the time, extended family live in a similar neighbourhood so neighbours are kin (usually, cousins), but this is not always the case.

5. Klein (2009) contrasts the time of utterance (i.e. the time at which the utterance is expressed), the topic time (i.e. the time about which something is asserted or asked) and the time of the situation (i.e. the time at which the situation obtains or occurs)

6. For this research I benefited from the help of Lorena Pool Balam.

7. Nyst (2007) mentions a similar strategy in Adamorobe Sign Language, which she calls 'growth-line.' 
8. Note that among Yucatec Mayas pointing to the moon in another form of time keeping (see Le Guen and Pool Balam, 2012, for details).

9. This is the gloss used by bilinguals for this sign.

10. The quotable gesture bin 'go' looks like the temporal gesture in Figure 2 . However, the upward gesture used with spatial reference is more refined in this domain, so speakers can vary meaningfully the height and the direction of the gesture, even the movement and the hand shape (see Le Guen 2011b for details).

\section{References}

Bohnemeyer, J.

2003 Invisible time lines in the fabric of events: Temporal coherence in Yucatec narratives. Journal of Linguistic Anthropology, 13(2). 139-162.

Bohnemeyer, J.

2009 Temporal anaphora in a tenseless language. In W. Klein \& L. Ping (Eds.), Expression of Time (pp. 83-128). Berlin, New York: Mouton de Gruyter.

Branson, J., D Miller \& I. G. Marsaja

2002 Sign languages as a natural part of the linguistic mosaic: The impact of deaf people on discourse forms in North Bali, Indonesia. In E. Winston (Ed.), Storytelling and Conversation Discourse in Deaf Communities, 109-148. Washington, D.C.: Gallaudet University Press.

Calbris, G.

1990 The Semiotics of French gestures. Bloomington, IN: Indiana University Press.

Casasanto, D., \& K. Jasmin

in press The hands of time: Temporal gestures in English speakers. Cognitive Linguistics.

Cooperrider, K., \& R. Núñez

2009 Across time, across the body Transversal temporal gestures. Gesture, 9(2). 181-206.

Dasen, P., \& R. C. Mishra

2010 Development of geocentric spatial language and cognition. an eco-cultural perspective. Cambridge Studies in Cognitive and Perceptual Development (No. 12) Cambridge: Cambridge University Press. 
De Jorio, A.

2000 Gesture in Naples and gesture in classical antiquity. (A. Kendon, Trans.). Bloomington, IN: Indiana University Press.

De Vos, C.

forthcoming Sign-Spatiality in Kata Kolok: how a village sign language of Bali inscribes its signing space. PhD Thesis. Nijmegen, The Netherlands: Max Planck Institute for Psycholinguistics.

Evans, N., \& S. C. Levinson

2009 The myth of language universals: Language diversity and its importance for cognitive science. Behavioral and Brain Sciences, 32(05). 429-448.

Fauconnier, G., \& M. Turner, M.

2008 Rethinking metaphor. In R. Gibbs (ed.), The Cambridge handbook of metaphor and thought, 53-66. Cambridge: Cambridge University Press.

Fischer, S., \& B. Gough

1999 Some unfinished thoughts on FINISH. Sign Language \& Linguistics, (2), 67-77.

Fridman-Mintz, B.

2010 Tense and aspect inflections in Mexican Sign Language verbs: A Cognitive Grammar approach to morphology and phonology in signed languages. Saarbrücken, Germany: LAP LAMBERT Academic Publishing.

Fusellier-Souza, I.

2004 Sémiogenèse des Langues des Signes: Étude de Langues des Signes Primaires (LSP) Pratiquées par des Sourds Brésiliens Unpublished doctoral dissertation. Université Paris 8, Saint Denis.

Gaskins, S.

1999 Children's daily lives in a Mayan Village: A case of study of culturally constructed roles and activities. In A. Göngü (Ed.), Children's engagement in the world: Sociocultural perspectives, 25-59. Cambridge: Cambridge University Press.

Gaskins, S. 2006 Cultural Perspectives on Infant-Caregiver Interaction. In N. J. Enfield \& S. C. Levinson (eds.), Roots of Human Sociality, 279-298. New York: Berg Publishers.

Goldin-Meadow, S. \& C. Mylander

1984 Gestural communication in deaf children: The effects and non-effects of parental input on early language development. Monographs of the Society for Research in Child Development, No. 207, 49(3). 
Hanks, W. F.

1993 Copresencia y alteridad en la practica ritual Maya (Copresence and alterity in Maya ritual practice). In M. L. Portilla, M. G. Estevez, G. G., \& J. J. Klor de Alva (Eds.), De Palabra y Obra en le Nuevo Mundo, Vol. 3, Siglo XXI (pp. 75-117). Madrid: España Editores, S.A.

Haviland, J. B.

1993 Anchoring, iconicity, and orientation in Guugu Yimithirr pointing gestures. Journal of Linguistic Anthropology, 3(1). 3-45.

Haviland, J. B.

2000 Pointing, gesture spaces, and mental maps. In D. McNeill (Ed.),

Language and Gesture, 13-46. Cambridge: Cambridge University Press.

INEGI.

$2010 \quad$ III Conteo de población y vivienda 2010. Mexico.

Johnson, R. E.

1991 Sign language, culture and community in a traditional Yucatec Maya village. Sign Language Studies 73. 461-474.

Keller, R.

1998 A theory of linguistic signs. Oxford: Oxford University Press.

Kendon, A.

1992 Some recent work from Italy on quotable gestures (Emblems). Journal of Linguistic Anthropology 2(1). 92-108.

Kendon, A.

1993 Space, time and gesture. Degrès, 1993. Vingt et unième année No. 74b. 3-16.

Klein, W.

2010 How time is encoded. In W. Klein \& P. Li (eds.), The expression of time, 39-82. The Hague: Mouton de Gruyter.

Le Guen, O.

2011a Speech and gesture in spatial language and cognition among the Yucatec Mayas. Cognitive Science, 35(5). 905-938.

Le Guen, O.

2011b Modes of pointing to existing spaces and the use of frames of reference. Gesture. 11(3). 271-307.

Le Guen, O., \& L. Pool Balam

2012 No metaphorical timeline in gesture and cognition among Yucatec Mayas. Frontiers in Cultural Psychology, July 2012, Volume 3, Article 271. 
Levinson, S. C.

2003 Space in language and cognition: explorations in cognitive diversity. Language, culture and cognition; 5. Cambridge: Cambridge University Press.

Liddell, S. K.

2003 Grammar, gesture, and meaning in American Sign Language. Cambridge: Cambridge University Press.

Marsaja, I. G.

2008 Desa kolok: a deaf village and its sign language in bali, indonesia. Nijmegen: Ishara Press.

McNeill, D.

1992 Hand and mind. Chicago: University of Chicago Press.

McNeill, D.

2003 Pointing and Mmorality in Chicago. In S. Kita (ed.), Pointing: Where Language, Culture and Cognition Meet, 293-306. Mahwah, NJ: Erlbaum.

McNeill, D., J. Cassell \& E. T. Levy

1993 Abstract deixis. Semiotica 95(1-2). 5-20.

Meir, I., \& W. Sandler

2008 A Language in Space: The Story of Israeli Sign Language. New York, NY: Lawrence Erlbaum Associates.

Núñez, R. E., \& E. Sweetser

2006 With the future behind them: Convergent evidence from Aymara language and gesture in the crosslinguistic comparison of spatial construals of time. Cognitive Science 30(3). 401-450.

Nyst, V. A. S.

2007 A descriptive analysis of Adamorobe sign language (Ghana). University of Amsterdam. Retrieved from http://dare.uva.nl/ document $/ 93469$

Pfau, R., \& M. Steinbach

2006 Modality-independent and modality-specific aspects of grammaticalization in sign languages (Vols. 1-24). Potsdam: Linguistics in Potsdam.

Poy Solano, L.

2011 Margina el sistema educativo a niños con problemas de lenguaje. La Jornada. México. Retrieved from http://www.jornada.unam. $\mathrm{mx} / 2011 / 07 / 05 /$ sociedad/036n1 soc 
Russo, T., \& V. Volterra

2005 Comment on "Children Creating Core Properties of Language: Evidence from an Emerging Sign Language in Nicaragua." Science, 309(5731), 56-56.

Sandler, W., I. Meir, C. Padden \& M. Aronoff

2005 The emergence of grammar: Systematic structure in a new language. PNAS, 102(7), 2661-2665.

Senghas, A., \& M. Coppola

2001 Children creating language: How Nicaraguan Sign Language acquired a spatial grammar. Psychological Science 12(4). 323328.

Senghas, A., S. Kita \& A. Özyürek

2004 Children creating core properties of language: Evidence from an emerging sign language in Nicaragua. Science 305(5691). 17791782.

Senghas, A., A. Özyürek \& S. Kita

2002 Encoding motion events in an emerging sign language: From Nicaraguan gestures to Nicaraguan signs. In A. Baker, B. van den Bogaerde, \& O. Crasborn (eds.), Cross-linguistic perspectives in sign language research. Hamburg: Signum Press.

Shuman, M. K.

1980 The sound of silence in Nohya: A preliminary account of sign language use by the deaf in a Maya community in Yucatan, Mexico. Language sciences, 2(1), 144-173.

Sinha, C., V. D. S. Sinha, VJ. Zinken \& W. Sampaio

2011 When time is not space: The social and linguistic construction of time intervals and temporal event relations in an Amazonian culture. Language and Cognition 3(1). 137-169.

Valli, C., C. Lucas, K. J. Mulrooney \& M. Villanueva

2000 Linguistics of American Sign Language: An Introduction (3rd ed.). Washington, D.C.: Gallaudet University Press.

Vapnarsky, V.

1999 Expressions et conceptions de la temporalité chez les Mayas yucatecos (Mexique). Unpublished Doctoral dissertation, University of Nanterre, Paris 10.

Zeshan, U.

2003 "Classificatory" Constructions in Indo-Pakistani Sign Language: Grammaticalization and Lexicalization Processes. In K. Emmorey (ed.), Perspectives on Classifier Constructions in Sign Languages, 113-142. Mahwah, N.J.: Lawrence Erlbaum Associates. 


\title{
Deaf signers in Douentza, a rural area in Mali
}

\author{
Victoria Nyst, Kara Sylla and Moustapha Magassouba
}

\section{Introduction ${ }^{1}$}

Over the past 5 decades of sign language research, studies have focused on the sign languages of national deaf communities, based in Western or Asian cultures (cf. Brentari, 2010; Pfau et al. in press). In addition to those sign languages, home signing, i.e. the signing of deaf children growing up in entirely hearing, non-signing environments, has been studied extensively (Goldin-Meadow, 2003). Again, the majority of these studies are done in Western or Asian cultures where deaf education is available. What many of the home sign studies have in common is that the interaction studied is deliberately kept free of sign language exposure, as a result of a speechcentred educational approach. Another important bias in our understanding of home signing is the fact that most studies are based on the home signing of children. More recently, a third type of signing has come under study, i.e. that found in communities with a high incidence of hereditary deafness, the topic of this volume. The findings emerging from these latter studies show significant structural differences with the sign languages of large, national deaf communities.

The current focus in sign language studies leaves two important questions unanswered. What do the sign languages of national deaf communities in Africa look like? And, what does the sociolinguistic situation and the signing of home signers on the African continent look like? This paper addresses the latter question. An important motivation for it is the fact that the majority of deaf Africans have no access to deaf education. It is likely that most of them are not in regular contact with a large, stable deaf community. As such, they are often qualified as home signers. However, little is known about these signers. $^{2}$ This is striking, as in fact the majority of deaf people in the world are in precisely this situation, i.e. isolated from large deaf communities, with limited (if any) access to health care or education. A pertinent question is the extent to which such people and their signing fit the labels presently available in our research vocabulary. The notion of 'home sign' in particular 
seems to require refinement. Also, knowing more about signers and their languages in areas without deaf education and large deaf communities helps us develop more reliable hypotheses about the diachronic development of sign languages, both young and old.

This chapter reports on a survey of deaf signers and signing communities in the administrative unit of the cercle of Douentza in central Mali, West Africa (see Figure 1). This survey was part of a larger effort to investigate signing in the wider Dogon area of Mali, roughly covering the cercle of Douentza and the neighboring cercles of Koro, Bankass and Bandiagara. With limited access to medical care, the incidence of deafness is relatively high (around 0.4\%, see below). Deaf education is not available. As such, the area is representative of most rural areas in Mali and West Africa more generally. Using snowball sampling, a deaf-led team of Malian signers traced and recorded deaf signers in Douentza. The deaf signers as well as selected hearing interlocutors or family members were interviewed about their deafness and interaction patterns. Although the observations are preliminary and often impressionistic, they do allow a basic assessment of the sociolinguistic settings of most signers and of how they compare to the types of sociolinguistic settings identified in the sign language literature.

The paper starts with an introduction of deafness and sign language in Mali in $\S 2$. In $\S 3$, the Dogon Sign Language Corpus and the sign language survey in Douentza are presented. The signing situation in seven places in the cercle of Douentza is presented in this section, followed by a discussion in $\S 4$ of the findings and their implications for our understanding of sign linguistics and a conclusion in $\S 5$.

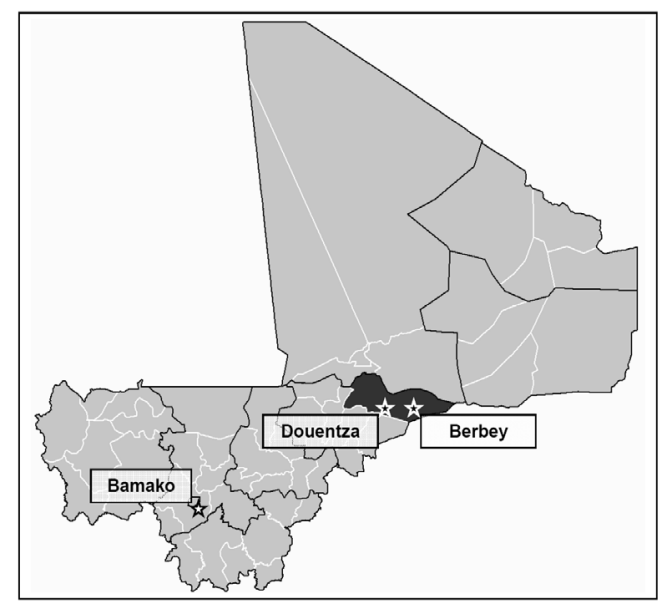

Figure 1. The map of Mali, with the cercle of Douentza shaded in black. ${ }^{3}$ 


\section{Deafness and sign languages in Mali}

No official figures are available for the incidence of deafness in Mali. Evaluating several studies done on deafness in West Africa, McPherson and Swart (1997) conclude that the prevalence of severe/profound bilateral hearing loss in West Africa is about three to four times the prevalence rate in industrialised nations. UNICEF (1985) gives an estimate of $0.5 \%$ for the prevalence of moderate to severe hearing loss in developing countries. If profound deafness is between $0.3 \%$ and $0.5 \%$ in Mali as well, this would mean the number of deaf Malians can be estimated to be between 40,000 - 70,000 given a total population of 14 million. The number of deaf Malians relying on signed communication is probably relatively high, as the availability of hearing aids and speech therapy is virtually non-existent.

The cercle of Douentza has a total population of 247,794 , with an estimated deaf population numbering between 700 and 1,300.

Mali is a multilingual country, both in terms of spoken languages (as will be discussed in \$2.5) and sign languages. A person's age and access to deaf education determines which sign language they are likely to use. Young, urban signers with access to education tend to use Langue des Signes de l'Afrique Francophone (henceforth LSAF), which is a variety of American Sign Language with influences from French and local signs and gestures. Older urban signers typically use the local Malian sign language, as do younger signers who live in rural areas and/or have no access to deaf education or to LSAF signers. Langue des Signes Française and Langue des Signes Quebecoise have been introduced to the Deaf community at some point in time, but these languages have had less lasting impact. No sign language has been recognised by the Malian constitution so far, but LSAF is the sign language used in education (see $\$ 2.3$ ).

\subsection{Malian Sign Language in Bamako}

The Ethnologue of the world's languages mentions the term Bamako Sign Language to refer to the variety of Langue des Signes Malienne as used in Bamako, the capital of Mali (www.ethnologue.org). The ISO code for this variety is [bog]. In speech and writing, the language is sometimes referred to as Langue des Signes Bambara. Because of the absence of a particular link between Bambara language and culture, I will use the more general term Malian Sign Language in this chapter, sometimes abbreviated as LaSiMa, based on the French name Langue des Signes Malienne. 
At present, only anecdotal information is available about the relation between the local sign language of Bamako and sign languages in other parts of Mali. Several deaf signers have made journeys in Mali and to surrounding countries, to meet other signers and learn new signs. They report no problems of mutual intelligibility inside Mali. Yet they also report differences in lexical signs, e.g. in monetary terms. Such variation is also found within the LaSiMa community in Bamako. Lexical variation is widely attested in many sign languages, where often each variant is centred around a deaf school (see e.g. Lucas et al. 2003 for ASL). The variation in Bamako is mostly centred around grins, places where deaf people, mostly men, gather in the afternoon to chat and have tea together, usually at the house or workplace of a popular deaf person.

Most sign language research in Mali has focused on the local sign language as used in the capital of Bamako. In 1999, the first and so far only dictionary of Malian Sign Language appeared, containing 570 signs as used in Bamako (Pinsonneault, 1999). In 2007, Projet LaSiMa was launched, aiming at the description and documentation of the LaSiMa variety of Bamako, funded by the Endangered Language Documentation Program of the Hans Rausing Endangered Language Project. The project, based at Leiden University and the Institut des Langues Abdoulaye Barry, in collaboration with AMASOURDS, was completed in 2010, resulting in a corpus of over 27 hours of recorded discourse in Malian Sign Language, featuring 65 signers (Nyst, Magassouba and Sylla, 2011). Recordings were mainly made in Bamako. In addition, about four hours of signing were recorded in Mopti, a town in the centre of Mali with a small signing community of about 10-15 deaf persons. The recordings in Mopti revealed that despite the absence of deaf education in this city, LSAF had found its way into the signing community, due to regular contact with deaf signers in and from Bamako. A more in-depth description of Malian Sign Language as used in Bamako, as well as its corpus, can be found in Nyst (2008; 2010).

\subsection{ASL and LSAF}

The deaf community in Bamako is shifting to ASL/LSAF. This sign language is referred to by the ASL sign for SIGN, whereby two upright index fingers make a backward, alternating, circular movement, palms facing to the front. The same sign also refers to the general notion of 'sign language'. The spread of ASL in Mali is part of a larger picture in which this sign language is used in deaf education in virtually all countries in West and Central Africa, albeit 
often alongside other sign languages (Nyst, 2010). Several factors have contributed to the spread of ASL in Deaf education in West Africa, especially the efforts of Reverend Andrew Foster. Between 1957 and 1987, he set up schools in 22 countries, mainly in West and Central Africa. In the 1980s, he attempted to set up a school in Mali, but failed.

Foster used Signed English with ASL signs in his schools (Oteng 1997). Considerable modifications took place in the case of ASL in Francophone countries in West Africa, whereby English loan elements were replaced with French elements (Kamei, 2006). These adaptations mainly concerned initialised signs, whose handshapes were changed so that they referred to the corresponding words in French instead of English. In addition, accompanying articulations or mouthings from English were replaced with French ones. Kamei (2006) argues this sign language is the result of a creolisation process and proposes the term Langue des Signes de l'Afrique Francophone (LSAF) for this variety of ASL. Despite the differences mentioned, signers of North American ASL tend to immediately recognise these varieties as being ASL-based. To what extent the ASL-based varieties have diverged from their source language and from each other remains to be determined. Malian signers of ASL/LSAF do not seem to perceive the two language varieties as different and use the same sign to refer to both. For a more extensive treatment of LSAF and ASL in (West) Africa, see Kamei (2006) and Nyst (2010).

\subsection{Sign language planning, facilities and endangerment in Mali}

Deaf schools are a crucial factor in the transmission of sign languages and in determining their status. Present and future deaf children in Bamako are likely to grow up using an ASL-based variety. Adult deaf signers too are eager to learn and use ASL and are often bilingual in LaSiMa and ASL. Having virtually no child users and a lower status compared to ASL, ASL is replacing LaSiMa in the Deaf community in Bamako and other places with deaf schools. Moreover, ASL is also spreading in places without deaf schools, e.g. Mopti, through contact with ASL signers in other parts of the country. As such, LaSiMa can be considered endangered.

Like many West African countries, Mali has no qualified sign language interpreters, neither for Malian Sign Language, nor for ASL. No regular sign languages classes are available for hearing people. The absence of such basic sign language facilities limits the participation of Deaf people in Malian society: deaf children do not have access to quality education as teachers do not have sufficient signing skills; Deaf organisations cannot lobby govern- 
mental and non-governmental bodies for their rights; and deaf children and adults have no access to vital information spread in public information outlets.

The multilingual sign language situation raises additional challenges. Educated Deaf signers, most of whom are active members of the Malian Deaf Association AMASOURDS, favour the use of ASL, whereas the vast majority of signers do not have formal education and use Malian Sign Language. Every initiative for setting up a sign language facility faces this language policy question and as mentioned before, several foreign NGOs have resolved this problem by introducing the sign language of their home country. Obviously, the introduction of foreign sign languages only adds to the existing challenges. At present, the Association Malienne pour les Personnes Sourdes does not have an official language policy. An extensive discussion of the distribution of local and foreign sign languages in West Africa is found in Nyst (2010).

\subsection{Deafness and sign language in Douentza}

Prior to this study, there was no information available about deafness in Douentza or the sign language situation there. Based on the total population, the number of deaf people in the Douentza cercle is estimated to be between 700 and 1300 (see §2). Currently, there is no school for the deaf in the cercle or in the wider region of Mopti. Due to the large number of deaf children encountered in the area surrounding Douentza, a deaf school is now being set up in Douentza. Currently, five deaf children are being taught at a mainstream school, awaiting the construction of the deaf school. The establishment of deaf education is likely to impact the sign language situation, as all Malian deaf schools use ASL at present. However, if the Malian policy of pédagogie convergente, in which children start primary education in their mother tongue before shifting to French, is extended to deaf education, the local sign language used in Douentza and surrounding communities may continue to be used. Blench (2005) encountered a small number of deaf signers in a Tebul Ure speaking hamlet in Koro, a cercle adjacent to the cercle of Douentza. He also recorded sample sentences and narratives in the sign language, in spoken Tebul Ure and in French. The deaf signers fluently communicated with the hearing people in their community. The Ethnologue has listed the language, and given it the ISO code [tsy] (www.ethnologue. com, 16/2/2012). 


\subsection{Spoken languages and language policies}

The official language of Mali is French. In addition, the Malian constitution recognises 13 spoken Malian languages as 'national languages', including Bamanankan, Fulfulde and Songhay, as well as one Dogon language, Toro So (www.ethnologue.com, 5/11/2010). Bamanankan (or Bambara, a Mande language from the Niger-Congo phylum) functions as a lingua franca and is spoken in varying degrees by $80 \%$ of the Malian population. For national languages, several facilities are provided, such as mother tongue education at the primary level, radio programs, weekly news bulletins on national TV, and newspapers. As mentioned above, the government supports the use of a bilingual approach in primary education referred to as pédagogie convergente, whereby children start off learning their mother tongue, and gradually shift to French (Traoré, 2001).

This paper results from sign language research in the Dogon area of Mali, an area about the size of the Netherlands which is highly multilingual. In his on-going survey and description project, Heath distinguishes 20 related Dogon languages and one isolate (www.dogonlanguages.org). The paper reports on a sign language survey in the town of Douentza and its surrounding communities. In Douentza, the Atlantic-Congo language Fulfulde is the lingua franca, but several other related Dogon languages are spoken in Douentza and the surrounding communities, including Jamsaytegu, Najdamba (Bondum-dom), Tommo-so, and Toro-tegu (Hochstettler et al. 2004). The report also includes one location a little north of the Dogon area, the village of Berbey. There, the Humburi Senni variety of Songhay is spoken (Heath, in preparation). The locations visited during the survey are presented in section 3.1.

\section{Dogon Sign Language Project: the Douentza survey}

In 2009, the project 'Documentation and Analysis of West African Sign Languages' was initiated at the University of Leiden, in affiliation with the Institut des Langues Abdoullaye Barry in Mali, aiming at the creation of a representative, digital corpus of the signing used in the Dogon area as well as a corpus of Adamorobe Sign Language, used in a village in Ghana (Nyst, 2007, Nyst, 2012a; Kusters, this volume). Data collection for the Dogon corpus was carried out in 2010 and 2011. Prior to this, a few major issues had to be tackled. The first was how to find and sample the set of signers contributing to the corpus. With very limited information about the incidence 
of deafness and signing in the area, in addition to limited resources, it turned out to be impossible to design a strategy that would lead to a statistically representative sample of deaf signers in the entire Dogon area. In addition, Nyst, principal investigator of the project, was not able to visit the field site. Fortunately, Magassouba had ample experience collecting sign language data in Bamako for the Malian Sign Language Project (2007-2010). In view of the above challenges and of a possible stigma associated with deafness, snowball (or chain referral) sampling seemed to best fit our survey aims. In snowball sampling, participants refer the researchers to other participants. This method is used in various types of studies where potential participants are hard to locate. During two visits, a team of signers, led by Magassouba, visited the Dogon area looking for deaf people in the urban centres who would then be asked to bring them into contact with other (deaf or hearing) signers. Obviously, this methodology has its constraints, for example a potential bias toward signers considered to be part of a (deaf) network, but it also brought about the possibility of new pathways and hence results.

During the first fieldtrip, the 'deaf snowball' brought the team to Mopti, where recordings were made as part of the earlier Malian Sign Language Project. Additional recordings were made during this visit as well. Consequently, the team continued to Bandiagara, and a few days later to Douentza, where it was based at the field station of the Dogon languages project led by Jeff Heath. In Douentza and surrounding communities, considerable numbers of deaf people were encountered. Two factors favoured the localisation of deaf people in and around Douentza. Firstly, with their extensive knowledge of Dogon communities, as well as practical help, the Dogon languages project supported the process of identifying deaf people in the area. Secondly, a young deaf man named Alassane Djepkele appeared to know an extensive network of deaf people. He was a vital link between the documentation team and deaf signers.

In Bandiagara, 10 deaf signers were filmed, and in Douentza, 19 were filmed, but the actual number of deaf people in Douentza was significantly higher. A considerable number of deaf signers in Douentza refused to be filmed. In the area around Douentza, 27 deaf signers were filmed in the six surrounding villages of Koubewel Koundia (10), Drimbé (4), Fombori (4), Ourodou Lamordé (3), Wakaraga-Na (3) and Madina (1). Inhabitants of these villages regularly visit the weekly market in Douentza to sell their produce and buy necessities. Some deaf people visit Douentza or other villages from time to time; others have never left their village (see $\S 3.2$ for a descrip- 
tion of the contacts between signers from the same and different places). During a second field visit, more data were collected with a selection of these signers. In addition, the team visited a reportedly 'deaf' family with six deaf members in the aforementioned village of Berbey, a Songhay village about 80 kilometers from Douentza.

For most deaf signers, interviews or narratives about their personal history or daily life were collected. In several cases, mainly those of young children who did not sign, deaf or hearing family or community members gave such accounts. In addition, for those signers with whom reasonable communication could be established, single signs were elicited using the lexical questionnaire developed by Parks \& Parks (2008), who also made available a set of accompanying images. As quite a number of pictures were not directly transparent in the Malian context, these were replaced with equivalent pictures taken in Mali, or by the real object represented in the picture.

\subsection{Results}

So far, the corpus contains 32 hours of video recordings, cut into 341 clips of varying lengths. After capturing the raw video data with Adobe Premiere CS5 and cutting them into sessions, the resulting AVI clips were compressed with TMPGEnc into both MPG1 and MPG2. Currently, 87 (25\%) of the clips are annotated at the gloss level. A lexical database is under construction. It is our aim to create a lexical database containing at least 200 signs, annotated for selected features pertaining to the phonology, semantics and iconicity of the signs.

The recordings, which were made in 13 locations (see Table 1 below), feature the signing of 41 men and 27 women. Out of these 68 signers, 59 are deaf, two are hard of hearing and seven are hearing. Of the deaf signers, 26 were reportedly born deaf, while 31 acquired deafness after birth. For two deaf signers, the time of onset of deafness was reported as unknown. The majority of deaf signers (54) did not make use of speech to communicate. Only two signers had ASL skills, following prolonged stays in Bamako. The youngest signer recorded was three years old at the time of filming, while the oldest signer was 80 . The average age was 30 . The signers recorded were born in 17 different places, with one deaf signer being born in Ivory Coast (see Table 1). 
Table 1. Places of birth and recording in the Dogon Sign Language Corpus

\begin{tabular}{|c|c|c|c|c|}
\hline Place & $\begin{array}{c}\text { Total } \\
\text { population }\end{array}$ & $\begin{array}{l}\text { Deaf signers } \\
\text { filmed here }\end{array}$ & $\begin{array}{l}\text { Deaf signers } \\
\text { born here }\end{array}$ & $\begin{array}{c}\text { Spoken } \\
\text { language(s) }\end{array}$ \\
\hline \multicolumn{5}{|c|}{ Cercle of Douentza } \\
\hline Douentza & 10722 & 19 & 12 & $\begin{array}{l}\text { Fulfulde, Jamsay } \\
\text { Tegu, Bondum } \\
\text { dom, Tommo So, } \\
\text { French }\end{array}$ \\
\hline Berbey & & 5 & 5 & Humburi Senni \\
\hline Fombori & 962 & 5 & 7 & Jamsay Tegu \\
\hline $\begin{array}{l}\text { Koubewel- } \\
\text { koundia }\end{array}$ & 1200 & 9 & 9 & Nadjamba \\
\hline $\begin{array}{l}\text { Ourodou } \\
\text { Lamorde }\end{array}$ & 603 & 4 & 3 & Nadjamba \\
\hline Madina & 300 & 2 & 2 & Nadjamba \\
\hline Diony & 225 & 0 & 1 & Nadjamba \\
\hline Diona & 1173 & 0 & 1 & Tommo so \\
\hline Drimbé & 635 & 5 & 5 & non Dogon \\
\hline Dala & ca. 800 & 0 & 1 & unknown \\
\hline \multicolumn{5}{|c|}{ Cercle of Ningari } \\
\hline Saré-dina & 1304 & 0 & 1 & $\begin{array}{l}\text { Pomuru, Bondun } \\
\text { dom }\end{array}$ \\
\hline Wakaraga-Na & 951 & 3 & 3 & Nadjamba \\
\hline \multicolumn{5}{|c|}{ Cercle unknown } \\
\hline Fanga & Unknown & 0 & 1 & unknown \\
\hline Darli & Unknown & 0 & 1 & unknown \\
\hline Fana & Unknown & 0 & 1 & unknown \\
\hline
\end{tabular}




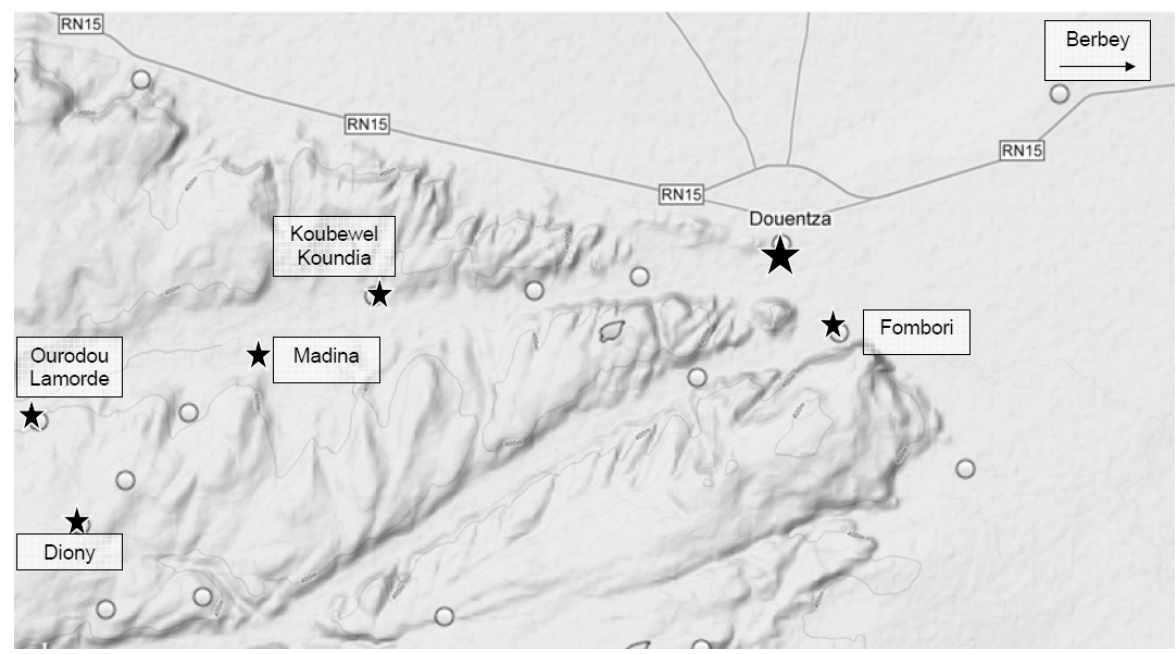

Figure 2. A map of Douentza and the villages where deaf people were encountered

Following approaches developed for earlier sign language corpora (e.g. the corpora for NGT (Crasborn et al. 2008), Auslan (Johnston 2008) and LaSiMa (Nyst et al. 2011), several types of data were collected for the Dogon Sign Language Corpus:

- personal narratives

- interviews about personal history

- signed guided tours by deaf signers around the house and outdoors

- elicited lexical data

- reports by the team members of the data collection

Metadata will be stored in the ISLE MetaData Initiative (IMDI), using the sign language profile (Wittenburg et al, 2000; Crasborn \& Hanke, 2003). The entire corpus, i.e. the video clips, annotations and metadata, will be stored in the archive of the Documentation of Endangered Languages program (DoBeS) at the Max Planck Institute for Psycholinguistics in Nijmegen. A web portal is currently under construction to inform the wider public about Malian Sign Language and the corpora compiled in the Dogon area as well as in Bamako. 


\subsection{Signers and signing communities encountered}

In this section, I present an overview of the signers encountered in Douentza and surrounding communities as well as in Berbey. As far as collected by the documentation team, information is presented about a) their sign fluency and b) their interaction with deaf and hearing people. Although this information is somehow anecdotal in nature, it serves to show that there is no one way to characterise the sociolinguistic setting of rural deaf signers and their signing skills.

\subsubsection{The town of Douentza}

The largest number of deaf signers was found in the town of Douentza, where 19 deaf signers were filmed. As stated above, there were also a considerable number of deaf signers who did not want to be recorded. The total number of deaf people encountered by the team in Douentza was estimated at around 35. This number roughly corresponds with the number of deaf people expected on the basis of a total population of 10.722 and an incidence of deafness of $0.4 \%$, which would be 42 deaf people. Strikingly, the survey team reported encountering more deaf people in Douentza than in the much larger regional capital of Mopti.

Although most deaf people do not meet on a regular basis, there are two or three places where a few deaf people do meet from time to time. The number of deaf people in Douentza fluctuates according to the season and the work that needs to be done in the fields.

There are two villages close to Douentza that were said to have many signers; Koubewel Koundia to the west and Fombori to the east. The survey team visited both villages. Koubewel Koundia turned out to have an especially high incidence of deafness. They also visited villages around Koubewel Koundia that were said to have deaf inhabitants. These surrounding villages include Ourodou Lamordé (three deaf people), Diony (one deaf person), Madina (one deaf person) and Wakaraga-Na (three deaf people).

\subsubsection{Fombori}

Fombori is a Jamsay speaking village a little to the east of Douentza. During the two visits to Fombori, the survey team found five deaf adults and three deaf children, in a total population of 962 . The oldest deaf person is a 
40-year-old man who contracted deafness as an adult. He now communicates very little, mainly with his wife and children, using signs. A 25-yearold cousin of his is also deaf. She is married and has three hearing children. Before her marriage, she spent some time in Bamako, as her father works there as an imam. She is a fluent signer and an active member of her family and the wider village community. Lastly, there are three deaf boys, aged between nine and eleven. They spend their time working in the fields and playing, and they communicate with their hearing and deaf friends using signs. All of the deaf people in Fombori were born deaf, apart from the aforesaid 40-year-old man.

\subsubsection{Koubewel Koundia}

Koubewel-Koundia is a Najamba (Bondum-dom) speaking village about nine kilometers west of Douentza, with a high incidence of deafness. It has 1,200 inhabitants, 10 of whom are deaf, i.e. $0.85 \%$. Deafness appears to have various causes here. Most deaf signers reportedly acquired deafness postlingually as a result of meningitis or another illness. Such infectious diseases are the primary cause of deafness in Bamako as well (Ag Mohamed et al. 1996). No close family relations were found to exist between deaf individuals, which makes a hereditary cause for deafness unlikely in most cases. Strikingly, all deaf signers are male and six of them are under the age of 10.

There are four adult deaf men. The youngest of them is 35 , and he is also the only adult who was born deaf. He is married and has fathered nine children, four of whom have died. He works in the fields in the morning, and in the afternoon he takes tea with his hearing friends. On Sundays, he goes to Douentza for the cattle market. He is also an active family man and a fluent signer. The other three men, aged 45, 60 and 66, all became deaf around the age of seven. The 45-year-old deaf man can understand spoken Najamba through lip-reading. He was married, but is now a widower with two hearing children. He has a limited social network, but extensive practical skills, including masonry, tailoring and waterhole construction. The two eldest men are both heads of large families. One of them has married two wives and has fathered nine children, five of whom have died. The other man has five children and enjoys spending time with them. Strikingly, both older deaf men reportedly have limited signing skills. None of the deaf men in Koubewel Koundia seek each other out for communication.

The six deaf boys in Koubewel Koundia are not close friends either. The oldest works in the fields all day and has no time to play with other chil- 
dren. His signing skills are limited and he uses relatively much pointing and gesturing to communicate. As such, his interaction patterns contrast sharply with those of eight-year-old Allaye, who has a large number of hearing friends he plays with regularly. He herds his family's cattle and runs small errands. He signs fluently and effectively communicates in this way with his friends and family. The youngest deaf boy is three. The survey team reported not having seen him sign. As for the other three deaf boys, no information is available at this point about their daily activities or signing skills.

Several hearing people were found to communicate effectively using signing. Most of them are family members of deaf signers, living on the same compound. The signing skills of the deaf signers vary significantly.

Some deaf signers of Koubewel-Koundia occasionally have contact with deaf people in Douentza and in surrounding villages, including the Najamba speaking villages of Diony, Madina and Ourodou Lamorde.

\subsubsection{Ourodou Lamorde}

Ourodou Lamorde is a village on the side of a hill, about three kilometers from Koubewel Koundia. Like the latter, it is a Najamba-speaking village. It has a population of 603 , three of whom are deaf. The eldest deaf person is an 80 -year-old widow, mother of 10 . She became deaf during her third pregnancy. She still mainly relies on spoken Najamba for communication, although her speech has become hard to comprehend. The two other deaf people are a father (50 years old) and his son ( 21 years old), both of whom have been deaf since birth. After the death of his first wife, the father remarried and has five hearing children from this second marriage. This deaf father is a man of principles and one of them is that he does not chat with his wife or children. The deaf son is from his father's first marriage, and he lost his mother when he was two years old. He works on the fields and herds the cattle. He is very timid and does not leave the village of Ourodou Lamorde. The deaf son communicates very little with other people and does not sign well. He is regularly visited by his deaf cousin from the neighbouring village of Diony.

\subsubsection{Diony}

When the documentation team came to Ourodou Lamorde, Bakaye was called for from Diony. He came with a hearing younger brother. Diony is 
a Najamba speaking village on a hill about 1-2 kilometers to the west of Ourodou Lamorde. There is only one deaf person in this village of 225 inhabitants. Bakaye, 21 years old, was born deaf. He is related to the deaf father and son in Ourodou Lamorde. Like other young men of his age, he works on the farming grounds during the rainy season and herds the cattle. Being the sole deaf person in the entire village, Bakaye seems to come closest to the definition of a home signer of all signers presented so far. From this perspective, the contrast between Bakaye and his deaf cousin in Ourodou in terms of signing skills is surprising. Bakaye has excellent signing skills and communicates well with the hearing people in his and neighbouring villages. He has his own signs to communicate, but also knows the signs used in Koubewel and Douentza, paying regular visits to these communities. His deaf cousin in Ourodou, on the other hand, has limited signing skills despite living with his deaf father.

\subsubsection{Madina}

Madina, a Najamba village with 300 inhabitants at 1.5 kilometers east of Koubewel, has one deaf inhabitant, a 23-year-old, married mother of one child. She was born deaf. Currently she is waiting to join her husband who went to work abroad four years ago. She is not very communicative, uses a lot of pointing and mainly converses with her friend, for whom she is employed as a domestic worker. In the cluster of Najamba-speaking villages, she seems to be the only deaf person who lives in an entirely hearing environment and has limited signing skills.

\subsubsection{Berbey}

Towards the end of the last field visit, the research team was informed of a deaf family in Berbey, at about $155 \mathrm{~km}$ east of Douentza and $5 \mathrm{~km}$ west of Hombori. Berbey, the Songhay village mentioned above, is situated on a hill close to Mount Hombori, the highest point in Mali. The villagers' spoken language is the Humburi Senni variety of Songhay (Heath,in preparation). Berbey has an estimated 450 inhabitants, five of whom are deaf.

The "deaf family" in Berbey consists of the families of two brothers, one hearing, one deaf, living on two neighbouring compounds. The hearing brother has married a hearing wife, and together they have one hearing and one deaf son ( 25 years and 22 years old respectively) and two deaf daughters 
(20 and 18 years old). The 25-year-old hearing son has a very good relationship with his deaf siblings and fluent signing skills. When the rainy season is over, he goes to Bamako to generate additional income. His younger brother was born deaf and works in the fields. He sometimes spends the dry season in Ségou to work there as a shoe polisher. He once visited Bamako. Their two younger sisters are also deaf. One of them sells juice at the Hombori market, and she has visited the Douentza market with her mother several times. When there is no market, she helps with household chores. She is very bright, has a good understanding of money and is very communicative. She is in charge of doing most of the shopping for the family. The younger deaf sister is 18 and helps her parents in the house and fields. She has never left the Hombori area. She is a good signer who spends most of her time with family members.

On the neighbouring compound lives the family of the deaf brother (50 years old), who also married a hearing spouse. He is a farmer, but during the harvest as well as market days he goes out to collect money for his family. He was away at the time the documentation team visited Berbey, and was still absent when the team visited again a few days later in the hope of meeting him. The deaf brother and his wife have a deaf daughter of 23 and a hearing son. The deaf daughter is the only child of the two families who has married, but she is now divorced. She has one child. She works in both the house and fields, and has been employed for two years in Mopti as a domestic worker. She seems to have some residual hearing and sometimes speaks, albeit unintelligibly. The documentation team did not report on the interaction patterns of the hearing brother.

Interviews with the family members revealed that the late father of the two brothers was also deaf. When asked about his signing, hearing family members told the documentation team that he signed like the divorced deaf daughter, i.e. with (unintelligible) voicing. From the spread of the deafness in the family, it seems apparent that the deafness is caused by a recessive gene, occurring in at least three generations. In fact, all deaf signers of the last generation were born deaf. The hearing and deaf children of both families get along very well and spend a lot of time together. All children are very competent signers. It was the impression of the documentation team that the signers of Berbey were the most fluent of all signers they had encountered during their visits to Douentza and surrounding villages. Lexically, there was also a significant divergence from the signing found in the latter places. 


\section{Discussion}

4.1. Findings of the survey project in Douentza: deaf villages, family sign language and fluent home signers

Having no deaf school or deaf community available, the situation in the cercle of Douentza can inform us about the interaction, communication strategies and general sociolinguistic circumstances of deaf people in areas with no access to education.

The signers and communities encountered during the survey in Douentza vary greatly. Some signers are very fluent and communicative, whereas others have limited skills. In the sign language linguistics literature, the number of deaf signers interacting on a regular basis is what distinguishes fully-fledged sign language from home sign. Interestingly, the variation in fluency in Douentza is not due per se to the number of deaf people found in a given community, or even in a given family. For example, Bakaye, the only deaf person in Diony, is a fluent signer. His deaf cousin Oumar, on the other hand, has limited signing skills, despite growing up with a deaf father. In other words, being the sole deaf person in a village does not mean one cannot become a fluent signer, and being a deaf child of a deaf adult does not mean one automatically acquires fluent signing skills. Similarly, the case of the deaf signers in Koubewel Koundia shows a not-so-straightforward distribution of skill, with some being very fluent and others much less so. The fact that the deaf signers do not seek each other out for communication suggests that the signing of older people does not represent the main language input for the younger generation. Rather, the signing of fluent and less fluent hearing family and community members is likely to provide the main language input. The case of the "deaf family" in Berbey, whose signing was judged by the survey team as the most fluent and expanded, does suggest however that transmission within a family can favor the acquisition of signing skills by individuals.

The signers and their communities also show striking similarities in one respect, namely that in all cases, hearing family or community members readily interact with deaf people using signs; there seems to be no stigma whatsoever attached to the use of gestures. Another notable feature is that all deaf adults are married, and many of them participate actively in family and community life. 


\subsection{Rethinking current labels}

The variation and similarities found in a) the social setting and b) the signing observed during the survey do not readily fall into the clear-cut categories of large Deaf community sign language, home signing or "village sign language". None of the sign languages found is used by a large Deaf community. In this section I reflect on how the signing described compares with home sign and the languages of communities with a high incidence of hereditary deafness. I argue that the term 'home signing' needs fine-tuning to be able to apply it to some of the signing in the corpus. The communities here also differ from those with a high incidence of deafness as a result of heredity, and I argue that instead of - or at least before - positing an increasing number of sign language types, it is more useful to describe and analyse a wider range of individual microcommunity sign languages. This enables one to properly appreciate the impact of the various idiosyncratic, sociolinguistic features.

\subsubsection{Home sign and home sign: the need for redefinition}

Despite the preliminary and impressionistic nature of the observations in the cercle of Douentza, three significant issues come to the fore.

Firstly, it becomes clear that the term 'home sign' needs rethinking. The main body of literature on home sign is based on deaf children growing up in hearing environments with no exposure to a conventional sign language, following oralist educational advice (c.f. Goldin-Meadow, 2003). The term 'home sign' is also used to refer to the signing of a deaf person who lives in a hearing environment in a typically rural area where deaf education and/or a signing community is not available (cf. Yau, 1992; Coppola \& Newport, 2005; Fusellier de Souza, 2006). The social settings of these two types of home signers are likely to differ quite radically in a number of ways, many of which are known to affect language and communication. It is likely that spending one's life in the same community where one is born, where literacy is no major factor in daily activities, and where gesturing/signing is considered the natural way of communicating with deaf people, favours the expansion and conventionalisation of the home signer's communication. Thus, the two types of home signing, oral and rural, are likely to differ significantly in their structural features.

Secondly, the role of hearing signers may be very different for these two types of home sign. As noted above, hearing signers seem to play a central role in the transmission of the signing to deaf children. This is all the more 
interesting as there seems to be a good deal of lexical consistency across the various signing varieties in the cercle of Douentza (and even in the signs used in Bamako). It appears worthwhile to consider the scenarios that may have led to this consistency. Is it the result of the irregular contact some deaf people have with each other? Or does mainstream hearing culture exploit an extensive, conventional vocabulary of gestures/signs to communicate with deaf people or others in the absence of a shared language? An analysis of the data collected will clarify how consistent the lexicon actually is. To study the nature and extent of the hypothesised conventional gesture vocabulary in mainstream hearing culture, new data need to be collected with hearing people in communities with and without deaf members.

Lastly, distinguishing between the home signing of children in the oralist tradition and that of adults in rural, gesture-prone environments raises an important question about the linguistic status of home signing. Generally, home sign is considered a system, rather than a language, because it does not fulfill two of the defining criteria formulated for human language, i.e. shared use across a user community and transmission across generations (Frishberg, 1987). The above findings suggest that these disqualifying features may be applicable to oralist home sign, but cannot be automatically generalised to rural home sign. Firstly, several home signers are fluent signers with no apparent communicative limitations outside the ordinary. Secondly, the evaluation of the defining criteria of a user community and intergenerational transmission with respect to rural home signing in Douentza is largely determined by the role of hearing signers. Deaf signers and their hearing interlocutors together form a community in which home sign is the means of communication. If indeed mainstream hearing culture avails of an extensive conventional gesture vocabulary, this implies it is transmitted across generations as well. Although the conventional vocabulary is hypothetical, and its relation to the actual home signing found is not clear, the current findings suggest intergenerational transmission cannot be ruled out either.

Furthermore, rural home sign varieties meet the criteria of a) a community of users, and b) transmission across generations for human languages as much as sign languages of large Deaf communities, albeit in different ways. Where it comes to the functionality for communicative purposes, rural home signing may differ categorically from oralist home signing in linguistic status.

Granting the signing of fluent rural home signers the status of language instead of merely a pre-linguistic system has important implications theoretically as well as practically. Considering these signers to be users of a full, natural language has important implications for the field of sign linguistics 
as well, in particular for our understanding of sign language genesis. All sign languages are assumed to have started out with one or more home sign systems. For sign languages of deaf communities that evolved in the context of deaf education, it is assumed that the first stage of the language was characterised by a merging of the various home sign systems contributed by the first children attending the school. This scenario has been documented in Nicaragua and Mauritius (see Kegl, Senghas and Coppola, 1999, and Adone, 2004 , respectively). For sign languages of communities with a high incidence of deafness, it is assumed that the initial input of the sign language consisted of the home signing of the first deaf person in the community. This process has been documented in the case of Al-Sayyid Bedouin Sign Language (see Kisch, this volume). However, if the home signing of the first generation of these new languages is actually a language, this implies that new sign languages actually present cases of radical language restructuring rather than of language genesis.

Practically, it is important that decisions concerning language policies, e.g. in deaf education, are informed correctly about the nature of the language varieties found in a given area. In the absence of a well-defined deaf community, linguistic studies on the signing of rural deaf signers are typically lacking. In such situations, the signing of rural deaf people is usually referred to as "home sign". If one infers the findings on oralist home sign in judgments on "home sign", the rural home sign languages risk being ignored as a suitable language for various applied purposes, including deaf education, which may lead to the introduction of foreign sign languages instead.

\subsubsection{Communities with a high incidence of deafness}

At least four of the signing communities in the corpus have a relatively high incidence of deafness, three of which were revisited during the second visit of the survey team: Koubewel Koundia, Fombori and Berbey. ${ }^{4}$ However, the distribution of deafness in all three communities differs from the communities with a high incidence of hereditary deafness (CHIDs) that are the topic of most chapters in this volume.

Deafness in Koubewel Koundia and Fombori is not mainly caused by hereditary deafness. In both villages, deafness has multiple causes and many signers became deaf postlingually, following a disease. This is likely to affect the patterns of language transmission. When deaf people are not bound by family ties, they are less likely to be exposed to the signing of deaf adults. Thus, the influence of hearing signers is likely to be even larger than 
in the case of CHIDs (cf. Nyst, 2007). Also, the high incidence of deafness in Koubewel and Fombori is likely to have a different historical pattern as compared to CHIDs. Berbey differs from Koubewel Koundia and Fombori in that the deafness there is hereditary, as in CHIDs. Unlike CHIDs, however, its occurrence has not spread to other families in the village community. If generations to come maintain an equally high incidence of deafness, the deafness and the sign language may spread out more widely, thus following the scenario of Mardin Sign Language (see the sociolinguistic description by Dikyuva, this volume) or other CHID languages. As mentioned, it was the impression of the survey team that the signing in Berbey was the most expanded and fluent encountered during the survey visits. It is probable that the frequent interaction provided by a harmonious family setting, with several deaf members of the same age group, stimulates the use and hence the expansion of the language.

It is likely that the sociolinguistic differences between Koubewel Koundia, Fombori and Berbey affect the structure of the signing in each, causing them to diverge from the signing in CHIDs, as well as from each other. Thus, we might want to refer to Koubewel Koundia and Fombori as "communities with a high incidence of non-hereditary deafness (CHINDs)" and to the sign language of Berbey as a "family sign language". However, rather than (or at least prior to) suggesting an increased number of sign language types, it is more useful at this point to describe and analyse a wider range of individual microcommunity sign languages, and thus to facilitate a proper appreciation of the impact of their various idiosyncratic and sociolinguistic features.

\section{Conclusion}

The survey in Douentza has resulted in a large annotated corpus of the signing of deaf people in various types of communities (Nyst, Magassouba and Sylla, 2012b). The corpus includes signing from villages with a high incidence of deafness, the signing of fluent and less fluent home signers, and a family sign language. From a methodological perspective, the survey showed that Deaf-led snowball sampling is an effective way to identify deaf signers in rural areas.

The preliminary analysis presented here suggests features of the signing community, such as the number of deaf people, the incidence of deafness, and the type of deafness (i.e. hereditary or non-hereditary), have sociolinguistic reflections. These reflections include the frequency and distribution of deaf-deaf and deaf-hearing interaction, the status of deaf people and hence 
their sign language, and the onset of sign language acquisition. The sociolinguistic features can safely be assumed to affect the structure of the sign languages through well-known linguistic processes, e.g. language acquisition and contact. The rich diversity of signers, languages and communities encountered during the survey, as well as the features they share (such as existing in areas with no deaf education and an average incidence of deafness) provide us with a natural laboratory to study how sociolinguistic setting interacts with modality in shaping visual language.

An important point that became clear is that the term 'home sign' is currently used to refer to the signing of deaf people in widely divergent and basically incomparable settings. To avoid overgeneralisations, it may be constructive to explicitly distinguish oralist home sign from rural home sign. ${ }^{5}$

The survey also encountered two communities that have a high incidence of non-hereditary deafness and a community that has a signing family with three generations of deafness. One can assume that these communities and their sign languages will differ from the existing categories of sign languages and/or signing communities, leading to two new types of sign languages: CHIND sign languages and family sign languages. However, in view of the dearth of knowledge about microcommunity sign languages and their communities, it is probably more effective to first systematically investigate an extensive range of such signing communities and the varieties in them to come to a deeper understanding of which sociolinguistic factors crucially influence the structure of signed languages.

\section{Notes}

1. We wish to thank the deaf and hearing signers who participated in this survey, Prof. Jeff Heath and his team of co-workers, Mr. Alassane Djepkele and the Institut des Langues Abdoullaye Barry for their generous and invaluable contributions to the survey.

2. The few studies focusing on adult home signers in rural areas include the work of Yau (1992); Coppola on Nicaraguan home signers (e.g. Coppola \& Newport, 2005); and Fusselier de Souza on adult home signers in Brazil (2006).

3. Image from http://upload.wikimedia.org/wikipedia/commons/7/79/Cercle_ of_Douentza.png. Date of download: 30/ 05/2012

4. Due to practical circumstances, Drimbé, with 5 deaf signers on a total population of 635 , could not be revisited. 
5. Zeshan (2010) uses the term 'communal home sign system' to refer to rural home signers in communities where hearing community members sign as well. The term 'rural home signer' is more neutral and does not make a statement about the interaction patterns with the hearing environment per se, but mainly serves to distinguish the term from home signing children in oral environments.

\section{References}

Adone, D.

2004 "Acquisition without a Language Model: The Case of Mauritian Home Sign". In: Alejna Brugos, Manuella R. Clark-Cotton, \& Seungwan Ha (eds.) BUCLD 29: Proceedings of the $29^{\text {th }}$ annual Boston University Conference on Language Development.12-23 Massachussets: Cascadilla Press.

Ag Mohamed, Soumaoro, Timbo \& Konipo-Togola

1996 Surdité de l'enfant en Afrique noire : cas de l'école des jeunes sourds de Bamako (Mali) In: Médecine d’Afrique Noire: 1996, 43 (11).

Blench, R. \& Mallam Dendo

2005a A survey of Dogon languages in Mali: Overview". OGMIOS: Newsletter of Foundation for Endangered Languages 3.02 (26): 14-15. http://www.ogmios.org/ogmios_files/266.htm

Brentari, D.

2010 Sign Languages: A Cambridge Language Survey. Cambridge, UK: Cambridge University Press.

Census

2009 Résultats Provisoires Nationales du RGPH 2009 http://instat.gov. $\mathrm{ml} /$ voir_actu.aspx?lactu $=44$

Coppola, M. \& E. L. Newport.

2005 Grammatical subjects in home sign: Abstract linguistic structure in adult primary gesture systems without linguistic input. Proceedings of the National Academy of Sciences 102(52) 1924919253.

Crasborn, O. \& T. Hanke

2003 Additions to the IMDI metadata set for sign language corpora. Agreements at an ECHO workshop, May $8+9,2003$, Radboud University Nijmegen. PDF manuscript. 
Crasborn, O., I.Zwitserlood \& J. Ros

2008 Het Corpus NGT. Een digitaal open access corpus van flimpjes en annotaties van de Nederlandse Gebarentaal. Centre for Language Studies, Radboud Universiteit Nijmegen. URL: http://www.ru.nl/ corpusngt/.

Frishberg, N.

1987 Home sign. In J. Van Cleve (ed.) Gallaudet Encyclopedia of Deaf People and Deafness (Vol. 3), 128-131. New York: McGraw Hill.

Fusellier-Souza, I.

2006 Emergence and development of signed languages: From a semiogenetic point of view, in Sign Language Studies 7:1, 30-56.

Goldin-Meadow, S.

2003 The resilience of language: What gesture creation in deaf children can tell us about how all children learn language. New York: Psychology Press.

Johnston, T.

2008 The Auslan archive and corpus. In D. Nathan (Ed.), The Endangered Languages Archive. Available at: http://elar.soas. ac.uk/languages. London: Hans Rausing Endangered Languages Documentation Project, School of Oriental and African Studies, University of London.

Heath, J.

1999 Grammar of Koyraboro (Koroboro) Senni, the Songhay of Gao. Rüdiger Köppe Verlag, Köln.

Heath, J.

in preparation Grammar of Humburi Senni, unpublished manuscript.

Hochstetler, J. L., J.A. Durieux \& E.I.K. Durieux-Boon

2004 Sociolinguistic Survey of the Dogon Language Area. SIL International.

Johnston, T.

2009 Creating a Corpus of Auslan within an Australian National Corpus. In Selected Proceedings of the 2008 HCSNet Workshop on Designing the Australian National Corpus, ed. Michael Haugh et al., 87-95. Somerville, MA: Cascadilla Proceedings Project.

Kamei, N.

2006 'The birth of Langue des Signes Franco-Africaine: Creole ASL in West and Central French-speaking Africa', in: Online conference paper of Languages and Education in Africa Conference (LEA2006). Oslo: University of Oslo 
Kegl J., A. Senghas \& M. Coppola

1999 Creation through contact: Sign language emergence and sign language change in Nicaragua. In M. DeGraff (ed), Comparative Grammatical Change: The Intersection of Language Acquisition, Creole Genesis, and Diachronic Syntax, 179-237. Cambridge, MA: MIT Press.

Lucas, C., R. Bayley \& C. Valli

2003 What's your sign for PIZZA? An introduction to variation in American Sign Language. Washington DC: Gallaudet University Press.

McPherson, B \& S. M. Swart

1997 'Childhood hearing loss in sub-Saharan Africa: a review and recommendations' International Journal of Pediatric Otorhinolaryngology 1997; 40:1-18.

Nyst, V.

2007 A descriptive analysis of Adamorobe Sign Language (Ghana) Utrecht: LOT.

Nyst, V.

2008 Documenting an Endangered Sign Language: Constructing a Corpus of Langue des Signes Malienne (CLaSiMa) In: Proceedings of LREC 2008, Sixth International Conference on Language Resources and Evaluation.

Nyst, V.

2010 Sign languages in West Africa. In Brentari, D. (ed.) Sign Languages: A Cambridge Language Survey, 405-432. Cambridge: Cambridge University Press.

Oteng, F. S.

1997 Deaf Adwoa Benewaa. Kumasi: Kumasi Catholic Press.

Parks, E. \& J. Parks

2008 Sociolinguistic survey report of the deaf community of Guatemala.

SIL International.

Pfau, R., M. Steinbach \& B. Woll (eds.)

in press Sign language. An international handbook (HSK - Handbooks of linguistics and communication science). Berlin: Mouton de Gruyter.

Pinsonneault, D.

1999 Lexique des signes utilisés par les sourds au Mali. Bamako : Editions Donniya. 
Traoré, S.

2001 La pédagogie convergente : son expérimentation au Mali et son impact sur le système éducatif. Monographies Innodata 6, UNESCO : Bureau International d'Education.

UNICEF

1985 UNICEF report on prevention of Deafness: hearing aids. London:UNICEF.

Wittenburg, P., H. Brugman, A. Russel, A. Klassmann, \& H. Sloetjes

2006 ELAN: a Professional Framework for Multimodality Research. In: Proceedings of LREC 2006, Fifth International Conference on Language Resources and Evaluation.

Wittenburg, P., D. Broeder, \& B. Sloman

2000 EAGLES/ISLE: A Proposal for a Meta Description Standard for Language Resources, White Paper. LREC 2000 Workshop, Athens.

Yau, Shun-Chiu

1992 Création gestuelle et début du langage-Création de langues gestuelles chez les sourds isolés. Hong-Kong: Langages Croisés.

Zeshan, U.

2010 Village sign languages - A commentary. In: Napoli. Donna Jo \& Gaurav Mathur (eds.) Deaf Around the World: The Impact of Language. Oxford University Press.

\section{Corpora}

Nyst, V.

2012a A Reference Corpus of Adamorobe Sign Language. A digital, annotated video corpus of the sign language used in the village of Adamorobe, Ghana. Leiden University Centre for Linguistics, Universiteit Leiden.

Nyst, V., M. Magassouba \& K. Sylla

2011 Un Corpus de Reference de la Langues des Signes Malienne I. A digital, annotated video corpus of the local sign language used in Bamako and Mopti, Mali. Leiden University Centre for Linguistics, Universiteit Leiden.

Nyst, V., M. Magassouba \& K. Sylla

2012b Un Corpus de Reference de la Langue des Signes Malienne II. A digital, annotated video corpus of local sign language use in the Dogon area of Mali. Leiden University Centre for Linguistics, Universiteit Leiden.

\section{Websites}

www.dogonlanguages.org 


\title{
Language ecological change in Ban Khor, Thailand: An ethnographic case study of village sign language endangerment
}

\author{
Angela M. Nonaka
}

\section{Introduction}

Still under linguistic typological investigation, 'village' sign languages are currently distinguished from other kinds of manual-visual languages by the particular sociolinguistic context in which they develop. This rare language variety develops in relatively small, face-to-face communities that exhibit considerable geographic and cultural variability but also exhibit remarkable structural and demographic similarity-i.e., significant numbers of deaf residents, high degrees of kin relatedness, labor-intensive economies, and low degrees of occupational and educational differentiation between deaf and hearing people. 'Village' (a.k.a., 'indigenous') sign languages are some of the world's least documented languages. Severely under-described, little is yet known about their characteristic linguistic features. Because they remain poorly understood typologically, it is sociolinguistic context and function that currently distinguish village sign languages from other manual-visual language varieties-i.e., 'national,' 'original,' or 'urban' sign languages (Woodward 2000, Zeshan 2004).

The first schema for categorizing diverse manual-visual language varieties was offered by James Woodward (2000). His tri-partite model identifies three major types of sign languages, which he terms 'national,' 'original,' and 'indigenous' sign languages. According to Woodward, a national sign language typically refers to the dominant sign language(s) of the national Deaf community of a given country. Original sign languages, which often pre-date development of a national sign language, are hypothesised to develop in areas where deaf people have regular and sustained opportunities to meet and converse (e.g., in market towns and urban centers). Indigenous sign languages are assumed to emerge de novo without contact with or influence from other sign languages. Although never overtly stated, Woodward's 
classification system is a hybrid model, one that integrates insights and assumptions from historical linguistics (e.g., aspects of areal and genetic linguistic typologies) and from sociolinguistics (e.g., language contact, variation, and use vis-à-vis speech communities).

Just as Woodward's model reflects his scholarly training in sociolinguistics and historical linguistics, subsequent critiques and alternative models are also indicative of their authors' intellectual expertise. For instance, the sociocultural anthropologist Erich Fox Tree has problematised implicit assumptions about language contact and language emergence in Woodward's model. Highlighting an all too common analytical trope in which indigeneity is falsely equated with isolation, Fox Tree's research (2009) illustrates the rich avenues for and effects of local lingua-cultural contact for sign language emergence and categorization. An alternative model has been proffered for classifying sign languages by the language typologist, Ulrike Zeshan (2004, 2006), who is endeavoring to develop a formal typology of manual-visual languages. Zeshan's evolving model works from very broad, preliminary correlations between social contexts/structures and linguistic features/structures. Her model initially divides extant sign languages into two broad categories: 'urban' versus 'village' sign languages. Like in any good linguistic typological study, (non)relationships between and among languages are then delineated based on robust, comparative, feature-based analyses.

Classificatory systems in general are epistemological grids that cut across different dimensions or qualities of target phenomena and are imposed for particular analytical purposes. Classification is always provisional, but good classification allows for distinctions and invites refinement. These dynamics are apparent in contemporary efforts to classify sign languages. The development and application, as well as the acceptance and establishment of classificatory nomenclature, are complex, often contentious, processes that emerge and change over time. Typologies are inevitably imperfect, but nonetheless useful. Whatever their shortcomings, each model discussed above makes an important and long overdue contribution to our collective knowledge of sign language diversity. In this publication, I have incorporated terms from both Woodward's and Zeshan's models which best describe the social dynamics that impact the endangerment of sign language varieties used to the village communities where they spontaneously develop.

An apparent hallmark of village sign languages is their widespread endangerment. Like other small language isolates, their local language ecologies are delicate. To date, however, relatively little is known about how and why this language variety is so widely threatened. Based on case study analysis of Ban Khor Sign Language (BKSL), an endangered village sign 
language in Thailand, this chapter examines the causes and consequences of language ecological change in Ban Khor. The local sign language is imperiled by a complex combination of macro-level forces and micro-level practices that include: rapid demographic and socioeconomic structural transformations; heightened contact with the national sign language and Deaf community; and shifting language ideologies and patterns of language use. This analysis, built on rich ethnographic data spanning more than a decade, illustrates the efficacy of a holistic anthropological approach for explicating village sign language vitality or vulnerability.

\section{Language Ecology and Endangerment of Village Sign Languages: An Overview}

Village sign languages are associated with an unusual kind of language ecology, one that motivates the etymology of their classification. Prototypically a language isolate, this type of language is found and used in restricted settings - namely, relatively small, face-to-face communities. Small-scale societies with indigenous sign languages have been identified around the world, across time and space (Bahan and Poole-Nash, 1996; Branson and Miller, 1996; Cumberbatch, 2006; Ferreiro-Brito, 1983; Frishberg, 1987; Groce, 1985; Kakumasu, 1968; Kisch, 2004, 2006; Kuschel, 1973; Johnson, 1991, 1994; Marsaja, 2008; Nonaka, 2007; Nyst, 2007; Sandler et al., 2005; Shuman, 1980; Torigoe et al., 1995; Van den Bogaerde, 2006; Washabaugh, 1978, 1979, 1981, 1986; Woodward, 1982).

As the individual chapters of this volume illustrate, each village and its local sign language are geographically, historically, and culturally unique. Yet there are also striking similarities in the language ecology(ies) ${ }^{1}$ of village sign languages, including: unusually high incidences of deafness in the population; high degrees of biological and/or non-biological kinship; laborintensive, non-industrial local economies; low intra-community educational differentiation between deaf and hearing people; and low intra-community occupational differentiation between deaf and hearing people. In addition to these shared structural features, there are also broad resemblances involving the socio-communicative function as well as the language ideologies and practices associated with village sign languages and their attendant 'speech/ sign communities' (Nonaka 2009). For instance, in the villages where local sign languages spontaneously develop, it is not uncommon to find: widespread fluency in the local sign language among hearing as well as deaf people; neutral to positive attitudes toward sign language and deaf people; 
successful integration of deaf people into the mainstream of village life; and minimal interaction and/or identification with national or international Deaf communities and "big D" notions of Deafness (cf. Woodward 1975; Lane 1984; Reagan 1995; Ladd 2003).

Another similarity among village sign languages is the fragility of their signature language ecology(ies). With a life cycle that is often abbreviated, this language variety is vulnerable to extinction. Village sign languages are known to arise quickly, within just one to two generations, or less than 100 years (Nonaka 2009; Sandler et al. 2005). Village sign languages' rapid emergence has made them a focal phenomenon in contemporary studies of language emergence and evolution.

Far less attention, however, has been paid to the fragility and endangerment of this particular language variety. Even village sign languages like Martha's Vineyard Sign Language, which was used for a century or more on an island community with a large sustained local deaf population (Bahan and Poole-Nash, 1996; Groce 1985), are susceptible to endangerment and often rapid disappearance. How and why that is the case is not yet entirely clear, but developing detailed accounts of their delicate language ecology(ies) is crucial for understanding, and where appropriate, reversing, the widespread endangerment of this manual-visual language variety.

Languages have routinely appeared and disappeared since time immemorial, part of a normal cycle of development, diversification through divergence, and perpetuation or decline. "Language change and language loss" of this sort "are inherent to all language situations" (Grenoble 2011: 27). In recent decades, however, languages have begun disappearing on an unprecedented scale and at an unparalleled speed - a magnitude and pace that threaten to further diminish linguistic diversity by disrupting linguistic differentiation through normal processes of historical linguistic change. Whereas in past millennia there was a continual process of contraction and expansion of linguistic diversity, "...the situation now is that linguistic diversity is simply being lost without being replaced" (Ash et al. 2001:19).

The primary cause of the current spike in widespread language death is 'language shift,' a trend in language (dis)use whereby speakers cease speaking their native language in favor of a more socially, politically and/or economically dominant one. It can happen gradually or quickly, unintentionally or deliberately, willingly or unwillingly. Language shift is a complex matter. Multiple variables (e.g., demographic, economic, environmental, historical, ideological, pedagogical, political, psychological, and social) operating simultaneously at different levels (e.g., micro and macro, as well as local, national, international or supranational), contribute to language shift. 
Investigation of the complex, multi-faceted phenomena of language endangerment and language shift demands a comprehensive approach, and in that vein, 'Language Ecology,' a.k.a. 'Ecolinguistics,' (e.g., Haugen 1972; Enninger and Haynes 1984; Fill and Mühlhäusler 2001) is a powerful paradigm for studying language endangerment and shift.

In any ecology the environment and its inhabitants are functionally linked in a dynamic system of interdependence. In language ecology studies, 'the term ecology is a heuristic metaphor-a tool helping researchers capture the complex relationships that obtain between varieties of speaking, speakers, and the world in which the speakers move" (Mühlhäusler 1997:4). Language ecology research centers on study of language and language use in context - more precisely in multiple contexts, nested and overlapping - that are historically situated and dynamic. The analytical power of the paradigm derives both from the social scientific acknowledgement and demonstration that "...language is not isolated from other social cultural and ecological factors but interacts with them. Such factors include those which are traditionally considered to be within the realm of linguistics such as the presence and use of other languages, as well as those which are not, such as economics, politics, and the physical and natural environment" (Grenoble 2011:30).

Language Ecology's breadth of analytical scope is well suited for examining the intricacies of language endangerment and language shift. Various academic (sub)disciplines ${ }^{2}$ invoke and use the paradigm, but there is a strong intellectual affinity between Language Ecology and Anthropology. In their theoretical orientations, both emphasise holism - its merits and applications for conceptualizing and organizing the study of language(s), speakers, and their use of language(s) in situ. Methodologically there is a deep resonance too, since most language ecology research adopts, in part or in toto, anthropology's hallmark methodology - ethnography - a grounded-theoretical approach based on in-depth case study analysis incorporating a combination of diverse qualitative and quantitative techniques (Fishman 1964, Sommer 1997). ${ }^{3}$

A growing number of case studies of endangered spoken languages around the world illustrate the utility of ethnographically-informed research for explicating the causes, processes and consequences of language shift (Gal 1979; Dorian 1981, 1989; Phillips 1983; Garrett 1990, 2005, 2006; Kulick 1992; Field 1998; Jones 1998; Fader 2006, 2007, 2009; Meek 2001, 2007; Paugh 2001; Howard 2003, 2004; Augsburger 2004; Hoffman 2007; Leonard 2007, 2008; etc.). To date, those studies have focused on imperiled spoken languages. Here, language ecological research is expanded to include a case study of sign language endangerment. 
Synthesizing diverse quantitative and qualitative data collected during the course of more than a decade of linguistic anthropological research, ${ }^{4}$ this chapter provides ethnographic case study analysis of Ban Khor Sign Language (BKSL), an endangered village sign language in Thailand. Historically, the language's development and maintenance have been sustained by a complex web of interwoven factors, including: close geo-spatial proximity, demography, hereditary deafness, socioeconomic organization, religious ethos, language socialization ideologies and practices, as well as interactional patterns of daily life. Recently, however, alterations in and to that delicate ecological balance have occurred, resulting in rapid language shift and endangerment of BKSL.

The remainder of this chapter provides in-depth description of language ecological change in Ban Khor. By explicating the particular nature of and reasons for those changes in the context of Ban Khor, Thailand, this case study analysis also illustrates the methodological and theoretical contributions of holistic ethnographic research for investigating and understanding the causes and consequences of the widespread endangerment of village sign languages.

\section{Ecological Conditions Supporting Development and Spread of Ban Khor Sign Language}

\subsection{Geo-spatial Proximity and Demography}

Ban Khor is a village in the northeastern Issarn region of Thailand, founded around 1883 by Khun Khor, a low-ranking Thai-Lao nobleman. Geographically, the community is small and is organised as a classic 'nucleated settlement.' Occupying an area of just 1.8 square miles, the village consists of a densely clustered residential core surrounded in all four directions by several miles of rice fields, streams, and forests that separate Ban Khor from neighboring hamlets. ${ }^{5}$

Ban Khor is a village like many others in northeastern Thailand: a Theravada Buddhist community of subsistence rice agriculturalists who supplement their daily diets by foraging or fishing, and who augment their annual incomes by conducting small-scale economic activities or working as seasonal migrant laborers outside the village. Save for the number of deaf residents, Ban Khor is demographically unremarkable. In 2003, there were 16 (formerly 18) native deaf villagers in a population of 2,741 (close 
to $0.6 \%$ ), a number that is numerically small but statistically significant, given that the expected incidence of congenital hearing loss is just $1 / 1,000$ or 0.1\% (Reardon et al. 2004: 8).

The linguistic anthropological impact of Ban Khor's deaf population, proportional to its size, has been great. The initial appearance of deafness beginning in the 1930s (Nonaka 2007:30-32) and its steady expansion in the population set the stage for the creation of a new sign language. Ban Khor Sign Language (BKSL) arose de novo around 75 years ago. It began as a home sign system among members of the family to whom the first two deaf individuals were born, but quickly became a full-fledged sign language that is now three generations deep, used by more than 400 people in diverse interactional contexts.

\subsection{Hereditary Deafness}

Ban Khorians know that deafness is more prevalent in their village than in other communities. Recognizing that it occurs across generations but only in certain families, they acknowledge deafness to be 'hereditary.' Rather than a biomedical phenomenon (e.g., a spontaneous, non-sex-linked genetic mutation transmitted in a dominant pattern underlying a syndromic form of deafness with variable expression), however, in Ban Khor, kamma phan (heredity) is understood to be a karmic matter - a consequence of barp (karmic demerit, misdeed, sin). While there is radical divergence at the level of ultimate causation between the genetic and the karmic explanations for deafness, there are also remarkable parallels between the two explanatory models. For instance, both posit: 1) some sort of intergenerational transmission; 2) clustering according to family bloodline; 3) phenotypic distinctions correspondent with differences in origin/cause of deafness; and 4) increased likelihood but imprecise predictability of individuals being born deaf.

Within the local karmic explanatory model, the appearance and persistence of deafness in two families is locally attributed to two different incidences of barp involving unnecessary cruelty to and killing of an animal. These acts were committed by two men whose respective children and grandchildren were subsequently born deaf. ${ }^{6}$ This "sins of the father revisited on the son" argument (Groce 1985; Hand 1980; Weiss 1980: 98-99), as it is referred to in the Judeo-Christian tradition, is known as the "cause and effect retribution" idea in the Buddhist-Hindu tradition (Pappu 1987; Prasad 1989; Roeder 2001; Uchikawa 1991). Formal theosophical debates to the contrary notwithstanding, in popular Buddhism, demerit transference and 
bad karma remain powerful and pervasive explanations for myriad suffering and misfortune.

Given its purported causal origin, hereditary deafness could have led to stigmatization and ostracization of deaf people, but in Ban Khor, it did not. Instead, the tendency has been toward inclusion and participation. Integration is apparent along several social and economic parameters, such as marriage, kinship, land ownership, education, occupation, and daily routines.

\subsection{Socio-Economic Organization}

In Thailand, individuals are generally free to choose their own marriage partners, and "there are no prescriptive marriage rules other than that which prohibits marriage between those who are living or have lived together in the same household" (Keyes 1995: 134). Within this system, historically, deaf Ban Khorians, both men and women, have married and formed families with their hearing counterparts rather than with other deaf villagers. There is no single, compelling reason that explains why this pattern developed, but anecdotal evidence suggests that it has been in part a pragmatic choice based on a projected communicative advantage, one that obtains differently than in most other language communities, hearing or Deaf. In the context of the Ban Khor speech/sign community, where many people can and do sign, three deaf villagers married to hearing partners independently mentioned communicative convenience as a positive reason for marrying their spouses. More specifically, they indicated that it would be convenient to have a hearing spouse in case a stranger came to their house or in case they had to conduct business outside of the village.

Kinship is bilateral cognatic (a.k.a. consanguineal), and inheritance is flexible, although ideally, "[rice] paddy fields should be divided equally among all children" (Mizuno 1971: 87). In 2003, a survey investigating the local work activities and daily routines of Ban Khorians was administered to all adult deaf villagers who were resident in the village at the time, their close family members and neighbors who sign, and a group of randomly sampled farmers in the community. The survey revealed remarkable similarity among all three groups. For example, for all those surveyed, wet rice agriculture was their primary activity. Almost all of the respondents also engaged in similar small-scale, supplemental economic activities locally in the community. Many villagers, especially men, worked seasonally outside of the village. The preference for equal inheritance, the inherent labor demands of wet rice agriculture and other local work activities, and the traditionally low educational 
level among Ban Khorians, regardless of audiological status, appear to have combined to encourage economic participation by deaf people whose daily routines evince a remarkably high degree of similarity with those of hearing villagers.

Integration of deaf people in Ban Khor has been motivated, not only structurally-functionally, but also ideologically by the notion of karma, which is part and parcel of larger cooperative ethos. Conceptually, karma is inextricably linked not only to barp, but also to bun or 'merit' (see Hanks 1962, Ingersoll 1975, Kirsch 1977, Keyes 1983a, 1983b etc.). In the Thai Theravadin tradition, "individuals frequently account for events and experiences in their lives in terms of their relative store of merit; all statuses, situations, and events can - potentially, at least - be interpreted and explained in terms of merit" (Kirsch 1977:246). In that context, the pursuit of merit-making and avoidance of demerit accumulation are active processes played out in the course of everyday life.

\subsection{Karma and the Moral Logic of Inclusion}

As a manifestation of social ideology and praxis, karma vis-à-vis hereditary deafness in Ban Khor has been something of a double-edged sword because, while deafness is attributed to misdeed and demerit, rejection of deaf people would easily constitute a new barp, whereas neutral to positive treatment of deaf people could be a means of earning merit. Thus, besides offering a causal explanation for the presence of hereditary deafness in Ban Khor, the cultural logic of karma provides a formidable disincentive for discrimination against deaf people, and a strong incentive for their social inclusion.

The appearance of 'hereditary' deafness in Ban Khor had profound implications for the village's sociolinguistic ecology since, before there were deaf people, there was no sign language in the community. Absent a timetravel machine, it is impossible to reconstruct precisely how Ban Khor Sign Language evolved, but this much can be surmised: BKSL appears to have emerged spontaneously in the home of the family into which the first two deaf people were born. ${ }^{7}$ The language, which arose out of communicative necessity, developed rapidly and began spreading widely throughout the community.

While deaf people were crucial for the emergence of BKSL, both deaf and hearing villagers have been vital for its maintenance. Unlike most speech communities, where deaf people are expected to make linguistic accommodations (e.g. learn to speak or write the dominant language or use 
an interpreter), in Ban Khor, significant linguistic accommodation is made by hearing people who acquire the local sign language. This unusual sociolinguistic dynamic - a hallmark characteristic of the language ecologies of communities where village sign languages develop - is crucial for the spread and maintenance of village sign languages.

\subsection{Language Socialization: Ideologies, Practices, and Patterns of Every- day Life}

The willingness of hearing villagers to learn and use BKSL is consistent with broader concessionary linguistic accommodations that they routinely make. With the exception of Thai, all of the vernacular languages spoken in Ban Khor are sociolinguistically marginal (Smalley 1994). The prospect of learning one more-BKSL - is unproblematic in a community where multilingualism is the norm. These two patterns of linguistic accommodation derive from more basic Thai patterns of enculturation that have been critical to the maintenance and spread of Ban Khor Sign Language.

As it is classically understood in anthropological language socialization studies, 'accommodation' refers to the tendency of adults in a society to adapt themselves, their language, and the interactional situation to the needs and abilities of the child. By contrast, 'non-accommodation' describes an expectation that children should adjust their communicative interactions to the requirements of the situation (Ochs and Schieffelin 1984). Comparative ethnographic research on language socialization recognises a cross-cultural continuum of accommodation versus nonaccommodation, and Ban Khor falls somewhere midway along that continuum.

"In their communicative interactions with babies, Ban Khorians tend to let them be. Infants are carefully monitored and lovingly attended, but if they are not nursing or in need of immediate attention, they are often left bundled in blankets under mosquito netting. Babies are seldom construed as conversational partners, although this changes as they grow."

Nonaka 2011:621

Accommodation is evident in the primary language socialization of children aged nine to twenty-four months. BKSL has a Baby Talk register. Its classic characteristics mirror those of Baby Talk in American Sign Language (Erting et al. 1990:105) and include: "(i) heightened affect, (ii) active physical stimulation of the child, (iii) signing more slowly than usual, (iv) signing close to the child to maximise visual attention, (v) signing on the child's body, and (vi) repetition" (Nonaka 2004: 754). Adults often talk/sign to 
toddlers using Baby Talk or other child-directed utterances. Playing peek-aboo and other linguistic games is common too.

By age three, however, use of Baby Talk with children ceases in Ban Khor. Child rearing in Thailand is permissive (Piker 1964) in most ways, save one-Thai society is quite hierarchical, and early on, children are socialised to begin adjusting their communicative interactions to conform to the cultural norms of hierarchy. They should be polite and demonstrate respect, both linguistically and nonlinguistically (Howard 2003).

Cooperation is also highly valued in rural Thai society. Ban Khorians expect and are expected to help one another; they do so often and, usually, reciprocally. Mutual assistance is extended to family members, neighbors, and friends, but also to community members at large. This cooperative ethos is manifested in everyday practices and cultural patterns of caregiving that have contributed to the maintenance and spread of BKSL. As will be demonstrated below, multiparty and flexible care giving influences language socialization.

In Thailand, the basic family unit is the nuclear family. As mentioned earlier, kinship and descent are bilateral and, ideally, postmarital residence is matrilocal. Upon marriage, the groom moves into his wife's natal home (or her mother's family compound), where the newlyweds live for a few years or permanently. Thus, when the new couple becomes parents, there is abundant social support.

Most children are born at home, and for a few days or weeks after giving birth the new mother is literally expected to 'lie by the fire' - a postpartum tradition that is both a curative practice and a rite of passage whereby a woman 'cooks/ripens' or fully matures (Hanks 1963). While she lies by the fire, the new mother is exempted from all work. Her only duties are to nurse the newborn, to drink special hot herbal water that promotes richer breast milk production, and to heal her genitals by washing with another special herbal water mix. During this period of recuperation, her husband and relatives assume all of her normal household responsibilities and also attend to the needs of the baby. Extended family members are in charge of bathing the child, changing and washing soiled clothing and bedding, arranging a Buddhist initiation and naming ceremony, and even taking the newborn to the health center to register its birth.

Distributed multiparty caregiving is the norm in Ban Khor. Once a child is weaned, it is quite common for others in the household (e.g. young, unmarried aunts or cousins) to assume primary childcare responsibilities. When they are slightly older, children sometimes choose to live in other homes in their maternal grandmother's compound or at the houses of other rela- 
tives. Flexible caregiving of this sort influences language socialization in interesting ways. For example, in several instances, a deaf aunt became the primary caregiver for a hearing niece or nephew who grew up to be fluent in BKSL.

As in other societies, Ban Khorian children are first exposed to the language(s) of their community at home. Many of the most fluent hearing BKSL signers are the close relatives (e.g. children or siblings) of deaf people with whom they live. Prototypical primary language socialization among co-resident family members is insufficient, however, to explain the spread of BKSL to 15-26 percent of all villagers within less than a century because in Ban Khor, there are only nine households with deaf residents, who total fewer than 20 village-wide. Yet, there are hundreds of people who can sign.

The rapid transmission of BKSL has not occurred randomly. Of those who know BKSL, 73 percent reside in the same area of the village where most deaf Ban Khorians live. Hearing signers also draw almost exclusively from one social class - they are farmers, as are all the deaf people and their families. Relatives and neighbors of deaf people are more likely to acquire BKSL. In short, there are clear correlations between a hearing person's proximity (e.g. relational links) to and interactions with deaf people and his/her signing ability - the closer and more frequent, the better (Nonaka 2009:221-225).

The rapid spread of Ban Khor Sign Language by way of close and repeated interactional proximity between deaf and hearing people has been sustained, not only by the socio-cultural structures, ideologies, and practices described above, but also by the organization and function of local economic life. Ban Khor's economy is marked by a high degree of labor intensity and a low degree of automation. Human labor is critical for economic survival there. Historically, the value of human capital has derived from practical experiential competence rather than from formal education. In that environment, deafness has posed no impediment to the performance of traditional work, and the nature of those activities, in turn, has encouraged the inclusion and participation of deaf people.

In Ban Khor, the overwhelming majority of residents are farmers. Traditionally, most have practiced subsistence wet rice agriculture, supplementing their daily diets through fishing and foraging, and augmenting their annual incomes through various small-scale economic activities like weaving, basket-making, gardening, herding water buffalo, and so forth.

Unlike other areas of the country, in Thailand's northeastern Issarn region, climate limits the number of rice agricultural cycles to one per annum. Farmers have a single opportunity to grow all the rice (the primary 
staple of their diet) required to feed themselves and their families for a year, and excess rice is sold for profit. The manpower needs associated with this form of agriculture are very high, and to meet those demands, villagers form nawan or labor exchange groups. Another manifestation of the Thai cultural ethos of cooperation, nawan are also important social networks within and through which deaf and hearing individuals interact and the latter acquire BKSL.

In recent decades, some people, especially men, have begun seeking employment outside the community. Some people leave Ban Khor for extended periods of time, but more typically, villagers choose to work a seasonal migration circuit. According to this pattern, they go to another province to pick rambutan fruit or to cut sugarcane for a relatively brief period of time (e.g., about one to two months), but always return to the village to resume wet rice agricultural work. Even while they are away from home, however, their primarily social and communicative interactions are with other Ban Khorians because, as short-term migrant laborers, they typically travel together in groups with fellow villagers, especially kinsmen and friends.

\section{Changing Language Ecological Conditions Contributing to the Decline of BKSL}

Although it has thrived for nearly a century, Ban Khor Sign Language is now imperiled. The causes of endangerment are complex - a combination of interwoven macro- and micro-level processes that include: dramatic economic transformations; marked social and demographic changes; and heightened contact with the national sign language and Deaf community that is changing local language ideologies and patterns of language use. Together, these forces are rapidly altering Ban Khor's language ecology and undermining the continued viability of BKSL.

Thailand has experienced profound and on-going politico-economic and social change during the last century and a half. As in other places in the world, processes of 'modernization' and 'development' have occurred not in linear fashion, but rather, in punctuated waves, the latest of which has exerted profound change even in far corners of the Thai countryside. No discussion of the full scope of those transformations is attempted here, save the most striking changes and their impact on the local language ecology. 


\subsection{Economic Transformations}

Wet rice agriculture remains the primary economic activity in Ban Khor, but farmers have increasingly moved away from subsistence to for-profit farming. In recent years, they have not only diversified their cash crops, which now include sweet corn and tapioca, but also have begun converting their rice fields into rubber tree farming plots. At the same time, the lumber industry has begun harvesting the forests of Ban Khor and surrounding communities. Deforestation has occurred rapidly and altered the traditional ecological balance. Many villagers continue to forage and fish for daily sustenance, but to do so, they must traverse ever-greater distances to exploit depleted natural resources. Thus, greater numbers of people now purchase foodstuffs.

In addition to food, an unprecedented number and variety of material objects are now available for purchase, not only in cities and market towns, but, to some extent, even within the Ban Khor community. Consumer products, large and small, are, for the most part, cheaper and more abundant than ever before due to the establishment of wholesale retailing, which has transformed supply chain networks throughout the country. Telecommunications products and services in particular have been utterly transformed. Similarly, transportation options - personal and public - have multiplied both in quantity and quality, dramatically increasing the range and frequency of villagers' mobility. More Ban Khorians of both sexes are now working outside of the village. Most still work the seasonal migrant circuit, although some commute, bi-weekly if not daily. While those who work outside the community still tend to travel in groups with other villagers, they do so in smaller numbers or sometimes not at all. All of these changes are part and parcel of the local shift from a subsistence economy to a complex cash economy. The latter has existed in Thailand for many decades, but the new supremacy of the cash economy in the hinterlands of the country underscores the depth and breadth of the transformation - one with subtle but crucial implications for the local language ecology.

In the past all Ban Khorians, save a handful of monks and a few civil servants, were peers 8 engaged in common daily activities, and the nature of local work indirectly fostered the spread of Ban Khor Sign Language. All traditional economic activities (e.g., wet rice agriculture, foraging, fishing, herding) were both highly labor-intensive and highly cooperative. Hearing loss posed no particular impediment to participating in those activities, and the inherent need for human labor encouraged social inclusion. The frequency and routinization of local economic activities kept villagers in close contact, encouraging regular deaf-hearing communicative interactions, which in turn 
provided opportunities for exposure to and acquisition of BKSL by a significant portion of local the population. ${ }^{9}$

By contrast, the dramatic economic changes now underway in Ban Khor are altering the traditional language ecological balance. There is growing differentiation among villagers with respect to their daily activities and their socioeconomic statuses. Projected over time, this trend, especially when combined with concurrent shifts toward greater educational and social differentiation, has adverse implications for the maintenance of BKSL.

\subsection{Social and Demographic Changes}

The traditional economic structuring of village life along with close residential proximity, a natural outgrowth of the nucleated settlement pattern, have optimised opportunities for deaf-hearing socio-communicative interactions, which in turn have supported language maintenance of Ban Khor Sign Language. Acceptance of BKSL into the community's language repertoire has also been supported by a complex constellation of demographic and social factors, such as: small population size and high kin relatedness, low educational differentiation among villagers, and a cooperative cultural ethos that encourages accommodation and inclusion. In addition to undergoing major economic transformation, the community of Ban Khor is also experiencing significant demographic and social changes that impact the local language ecology-loosening traditional community bonds and social ties that have sustained BKSL.

The population of Ban Khor has grown steadily and significantly over the last decade. Ban Khorians' social networks now include more people from outside the community, a fact illustrated by the significant increase in new surnames found in village registries. Hearing outsiders who marry into the village do not know BKSL and are less likely than in the past to interact with deaf people because of population growth and also because of increasing occupational and educational differentiation between deaf and non-deaf individuals.

When research commenced in Ban Khor, circa 1996, the average educational level among hearing people in the community was at the primary sixth (P6) grade level, although officially, education had recently been made compulsory for nine years. Subsequently increased by an additional three years, the local village school system now provides a full six-year (M6) high school education. Historically, there have been no local opportunities for formal schooling for deaf people. 
Formal deaf education ${ }^{10}$ began in Thailand after the Second World War, with the opening of The School for Deaf Children at Dusit District, Bangkok, the forerunner of the institution now known as Sesathien School for the Deaf, which was established in the 1950s. Deaf education was centralised in Bangkok for a number of years, but gradually, residential schools were built in other regions of the country, including in the northeastern Issarn region. Until recently actual attendance rates have lagged or fluctuated, however, especially in rural areas like Ban Khor.

In 2000, no deaf Ban Khorians over the age of 25 had any formal educational training, although all those under that age either were enrolled in or had attended, if only briefly, special deaf schools. Today, all eligible children attend residential deaf school beginning in elementary school. To get an education, deaf Ban Khorians must leave their community and enroll in boarding schools that are located several hours away by car. They reside there for months at a time, returning home only a few times per year. The language of instruction and of social life at those schools is Thai Sign Language (TSL), the language of the country's national Deaf community, which is used by an estimated 56,000 deaf people in Thailand (Reilly and Suvannus 1999). At school, Deaf children quickly acquire TSL and then introduce it back into their home village's language repertoire.

\subsection{Contact with Thai Sign Language}

A decade ago, Ban Khorians were keenly aware of and quick to point out differences, especially lexical ones, between their local sign language and other manual-visual languages. In 1996, when study of Ban Khor and its sign language began, there were striking differences between core vocabulary signs in BKSL and TSL, and lexico-statistical analysis of comparative data from the two languages, collected using a modified version of the Swadesh list, underscored the fact that Ban Khor Sign Language and Thai Sign Language were distinct, genetically unrelated languages (Woodward 1996, 2000). Hence, there are significant differences between BKSL and TSL across multiple lexical domains, for example, in kinship terminology, colours terms (Nonaka 2004), toponyms (Nonaka 2007), numbers, days of the week, months of the year, foods, fruits, vegetables, animals, tools, and so on.

Over the course of the last decade, however, there have been many observable changes occurring in Ban Khor Sign Language's vocabulary. 
Those alterations have primarily involved contact-induced change in the form of borrowing from Thai Sign Language. At first (circa 2000-2003), lexical borrowing from TSL into BKSL happened gradually, and it primarily involved incorporation of vocabulary for which there were no existing words in the local sign language. This was most evident in the lexical domain of toponyms but also in the targeted borrowing of category words like 'work' or 'animal.' By 2008 there was a marked increase in the appearance of TSL lexical items in BKSL in many vocabulary domains, although in the course of actual conversation the expression of a borrowed TSL word was often accompanied by the original BKSL counterpart sign. Within the last three years, however, the rate and scope of vocabulary borrowings from TSL into BKSL has increased dramatically across virtually all lexical domains, including in core vocabulary.

Lexical changes notwithstanding, there remain other clear differences between BKSL and TSL. With regard to phonology, for instance, BKSL does utilise all of the "B-A-S-C-O-1-5" universal handshapes, a basic set of unmarked handshapes that are predicted to occur in all natural human sign languages, but the BKSL's phonological inventory also includes some less common phonological forms (Nonaka 2004, 2007) ${ }^{11}$ not found in TSL. As an illustration of morpho-syntactic differences between the village and national sign languages, compare and contrast their interrogative systems, especially content questions and the size and structure of their respective question word paradigms. Whereas TSL has six distinct $W h$-signs (Suwanarat et al. 1990), BKSL's entire $W h$-system is organised around just two signs (Nonaka 2010).

Figure $1-\mathrm{a}$ modified conversation analysis (CA)-style transcript ${ }^{12}$ with added visual frame-grabs of spontaneous signing and cultural metacommentary in Ban Khor Sign Language-provides a partial illustration of the breadth of discourse structures and practices found in BKSL. This narrative was recorded at the Ban Khor Health Center in 2002 as part of an elicitation session which adopted Mercer Mayer's (1980) children's storybook, Frog, Where Are You ${ }^{13}$ The participant is a deaf, male, native Ban Khor signer. Embedded within the transcript there are also examples of TSL contact-induced linguistic borrowing and change in BKSL. Both BKSL and TSL glosses are capitalised, with the latter also being italicised. English, utterance-level translations are provided above the embedded photos. 
1) Foreign (.) that's foreign

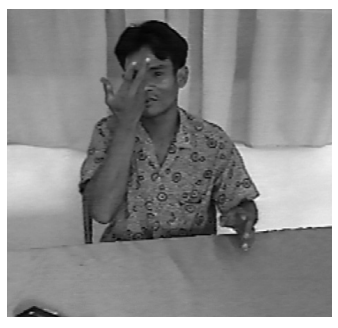

FOREIGN

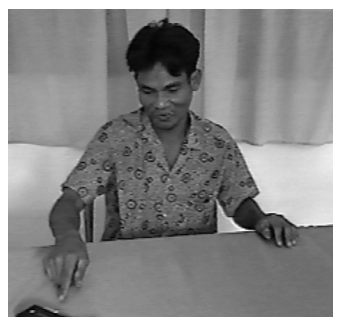

THAT

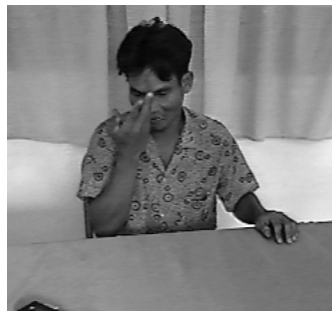

FOREIGN

2) (Here) there are lots of frogs

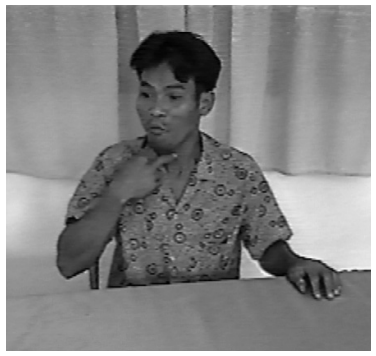

FROG

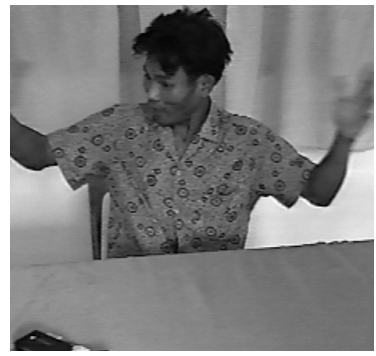

LOTS

3) (We) hunt them over there

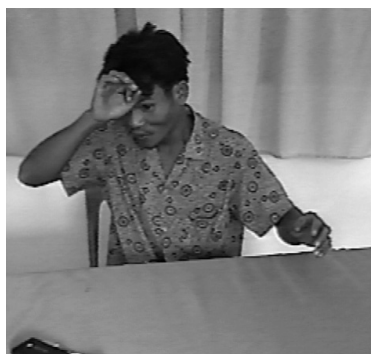

(night-)HUNT*
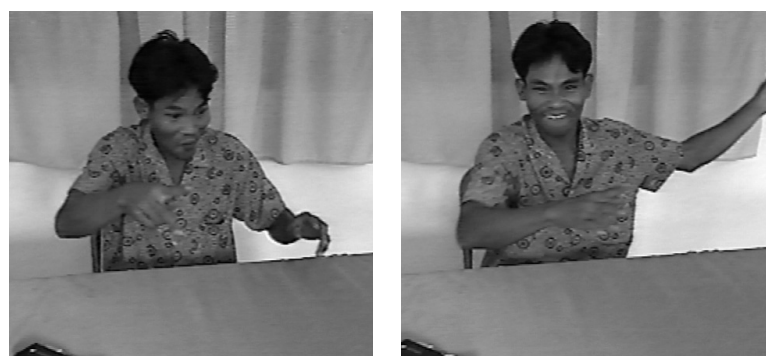

OVER THERE

*There are several distinct signs for different types of hunting in BKSL. The one depicted in Line 3 refers to night hunting and etymologically derives from the fact that local night hunters utilise a special light—fai song gop — that is strapped around the forehead and powered by a battery pack worn around the waist (see Figure 2). 
4) ((points to the storybook)) There, though, they love them

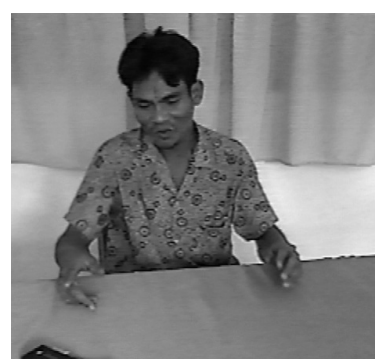

THERE

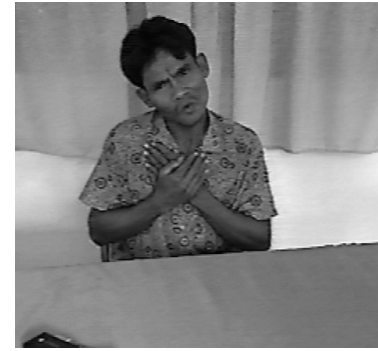

LOVE (intense)

5) I didn't know

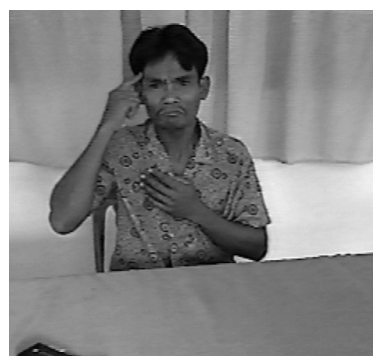

NOT KNOW

6) I didn't know they love them

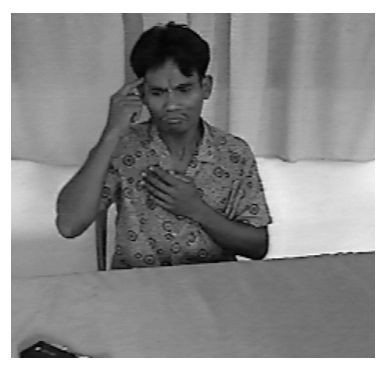

NOT KNOW

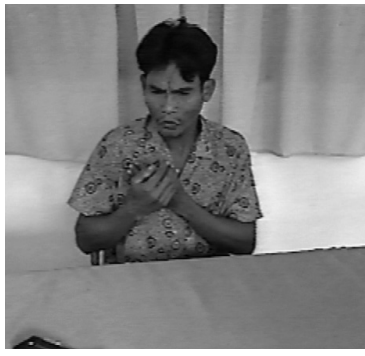

LOVE 
7) We chop off its head, grill the frog, and eat it

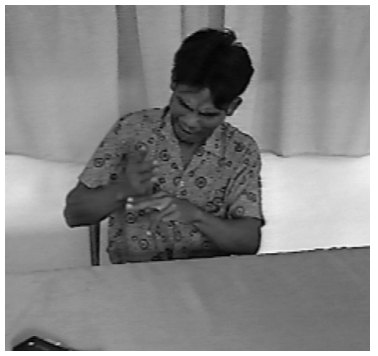

CUT (classifier)

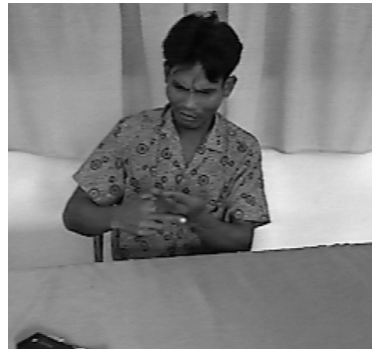

GRILL (classifier)

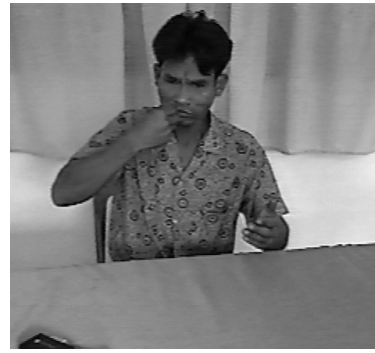

EAT

8) ((rests his head in his hand, hiding his face and laughing embarrassedly))
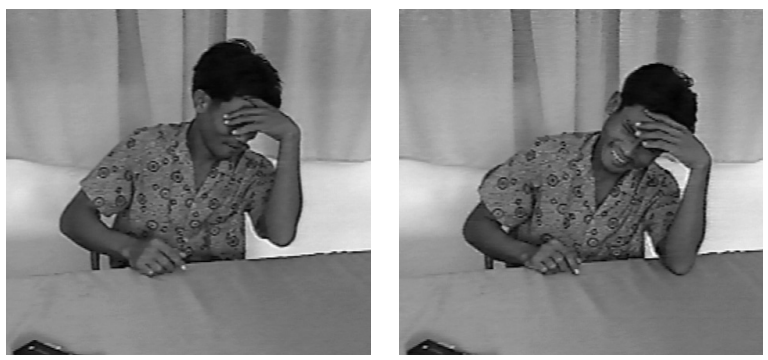

Figure 1. Foreigners Love Frogs but We Hunt and Eat Them

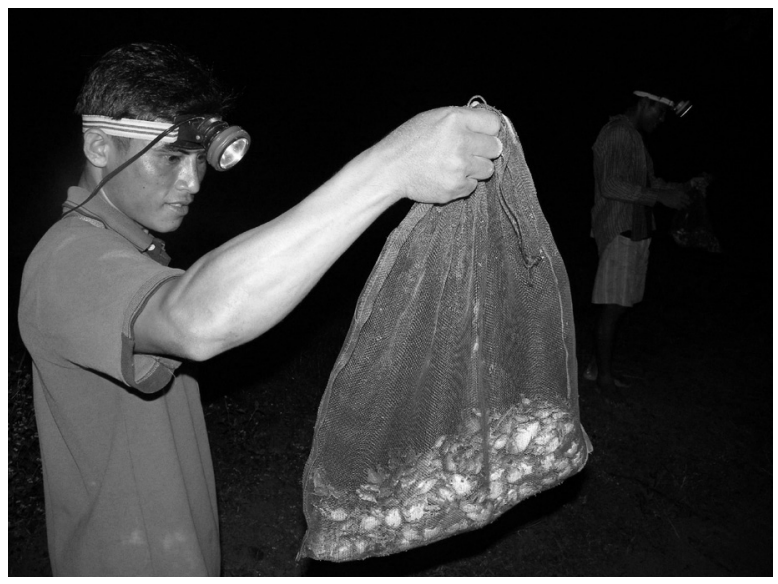

Figure 2. Man wearing fai song gop, a special light worn on the head for night hunting of frogs ${ }^{14}$

The preceding excerpt spontaneously transpired immediately after the linguistic consultant finished narrating the frog story. In this example the 
consultant contrasts cultural models about the treatment of frogs: the foreign perspective (Lines 1, 4 and 6) of frogs as loveable pets versus the local Ban Khorian view (Lines 2, 3, and 7) of frogs as a source of food to be hunted and eaten. In offering this cultural meta-commentary, the signer uses complex grammatical and logical structures (e.g., compound predicate $=$ Line 7); engages in multi-faceted perspective-taking (e.g., Line 4 and 6); and offers cross-cultural comparisons (Lines 2-3 and 7 vs. Line 4) and assessments (Line 8).

Embedded within the transcript in Figure 1, however, there is also evidence of contact-induced linguistic borrowing and change. The first instance of intra-sentential code-switching appears in Line 2, where the man uses the TSL sign for FROG, which is articulated at the throat with a V-handshape that moves in and out twice in rapid succession (see Figure 3). FROG in BKSL, by contrast, is made in neutral signing space with a bent 5-handshape, palm-oriented downward, that 'hops' up and down (see Figure 4).

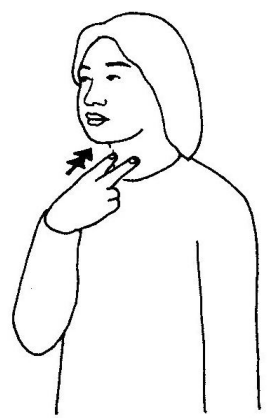

ท่ามือที่หมายถึง “แพะ” บางครั้งอาจมีนิ้วที่ตรง และส่วนแรกอาจดูเหมือนท่ามือ "กบ" แต่นิ้วจะ สัมผัสกับหน้าผากด้วย (เขาสัตว์) และโดยปกติ มีนิ้วที่งอ โปรดดูท่ามือ 1090.3

frog

Signs for "goat" may sometimes have straight fingers, and the first part may look like "frog," but they also touch the forehead (horns) and usually have hooked fingers. See sign 1090.3.

Figure 3. FROG in Thai Sign Language*

*Excerpted from The Thai Sign Language Dictionary (Suwanarat et al. 1990: entry \#974.1) Reprinted with permission of the authors.

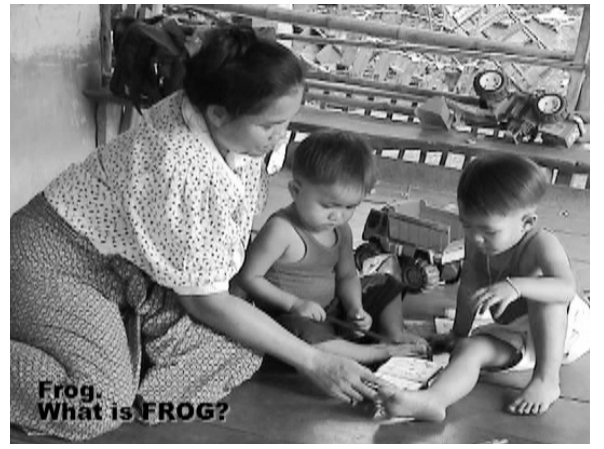

As demonstrated by the little boy (age 3) in white shorts, the sign FROG in BKSL is made as follows:

Handshape = 5-handshape

Palm orientation $=$ down

Movement $=$ hand 'hops' up and down

Location

$=$ neutral space (a.k.a. 'zero zone')

Figure 4. FROG in Ban Khor Sign Language 


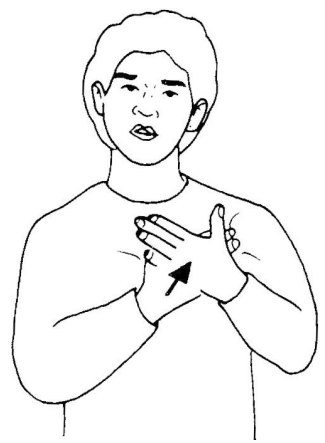

Figure 5. Thai Sign Language lexeme LOVE*

*Excerpted from The Thai Sign Language Dictionary (Suwanarat et al. 1990: entry \#168.2) Reprinted with permission of the authors.

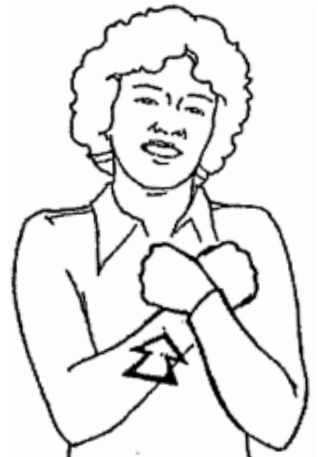

Figure 6. American Sign Language lexeme LOVE*

*Excerpted from A Basic Course in American Sign Language (Humphries et. al 1985:42)

Reprinted with permission of the authors.

Another interesting example illustrating the complexities and impacts of language contact appears later in the transcript. The lexeme LOVE in BKSL is made with a manual hugging gesture in neutral signing space, accompanied by an obligatory constellation of non-manual markers that include: a slight head tilt, pleasant facial expression, and eyes narrowed or closed depending on the intensity of affection. In Lines 4 and 6 of Figure 1, however, the native BKSL speaker produces a distinctly different sign. It is the TSL lexeme LOVE (see Figure 5) which itself not only resembles but is cognate ${ }^{15}$ with the American Sign Language (ASL) sign LOVE (see Figure 6). Although it is geographically counter-intuitive, TSL and ASL are in fact related languages that belong to the same language family (Woodward 1996). It is ironic that language contact, followed by rapid pidginization and creolization, of ASL and moribund and extinct indigenous and original sign language varieties in Thailand produced modern standard TSL (Woodward 2000), which now itself poses a threat, through language contact and language shift, to the continued viability of BKSL.

\subsection{Contact with the Thai Deaf Community}

Ban Khor Sign Language is rapidly being supplanted - and thereby endangered - by Thai Sign Language. Besides entering the Ban Khor speech/sign community through the formal schooling of young deaf children, TSL is also penetrating the village by several other means. For instance, new community outreach education initiatives promote the national sign language by 
distributing free visual TSL dictionaries to adult deaf Ban Khorians and their co-resident extended family members. As another example, local civil servants (e.g., public health officials, police, and teachers), most of whom moved to the village for purposes of work and therefore do not know BKSL, sometimes attend external training workshops where they learn 'sign language'which is always the national sign language - in order 'to be able to communicate' with deaf people in Ban Khor.

Televised sign language interpreting is yet another means by which the national sign language is being introduced into the village, and both deaf and hearing Ban Khorians, more of whom own and watch televisions than ever before, are being exposed to Thai Sign Language. ${ }^{16}$ Currently, the National Broadcasting Services of Thailand (NBT), Channel 11, only provides daily interpretation of news programs as well as regular coverage of parliamentary sessions, although the volume and variety of interpreted programming will inevitably expand as the number of professional interpreters grows. A national priority of the Thai government, ${ }^{17}$ significant expansion of interpreting services is already underway, with unintentional but adverse consequences for Ban Khor Sign Language, since development of interpreting services only pertains to sign language interpretation in TSL.

Greater contact with the national Deaf community, however, is the primary reason for TSL's growing influence in Ban Khor. Until the late 1980s deaf Ban Khorians' social networks were anchored in and circumscribed to their home village; they rarely met or interacted with other Deaf people. Throughout the 1990s contact with the Thai Deaf Community was still quite limited, except for a few young deaf Ban Khorians who temporarily left the village, briefly attended residential deaf school, but soon returned home.

Now, in the early $21^{\text {st }}$ century, the frequency, duration, as well as the quality of contact between deaf Ban Khorians and other Thai Deaf people has increased dramatically. Expanded transportation opportunities make it possible for deaf Ban Khorians to attend activities away from home that are sponsored by the National Association of the Deaf in Thailand (NADT). Additionally, deaf Ban Khorians are also seeking long-term employment outside the village, and when they work away from home, they almost always work and/or socialise with TSL-signing Deaf people.

Contact with the national Thai Deaf community is even altering marriage patterns in Ban Khor. Traditionally, deaf villagers of both sexes only married local hearing residents from their own community. That began to change, however, in 2002 when deaf Ban Khorians began choosing native TSLsigning Deaf spouses from distant communities. In the decade between 2002-2012, all but three deaf Ban Khorians were age-eligible for marriage. 
Seven (five males and two females) have never married. Eight have wed, however, and in two instances subsequently divorced and remarried. Of those eight deaf Ban Khorians who have married, half continued the tradition of marrying endogamously to hearing members of their own community, while the other half (re)married exogamously, that is, to TSL-signing Deaf individuals from other villages. Significantly, of the four mixed signing couples (e.g. a native BKSL signer and a native TSL signer), three have established post-marital residence in Ban Khor.

Inevitably, these changes have altered the balance of the local language ecology in Ban Khor, intensifying language contact and triggering language shift. During the last decade, lexical borrowing from TSL into BKSL has risen dramatically, and code-switching has started too. What is striking is that both linguistic borrowing and code-switching are being led by deaf Ban Khorians. Hearing village signers, by contrast, have been much slower to adopt TSL signs. Thus, contrary to existing theories of language maintenance of national sign languages, in Ban Khor, hearing signers are becoming the critical 'keepers' of BKSL (Nonaka 2009).

For deaf Ban Khorians, TSL is both a lingua franca (for communication with other deaf people) and a prestige code (for potential social, economic, and political mobility). Hearing villagers, by contrast, have no incentive to learn the language. Their continued use of BKSL is slowing language shift, but is unlikely either to prevent or reverse it because hearing people acquire sign language in order to communicate with deaf Ban Khorians, who are increasingly motivated to learn TSL due to its relative sociolinguistic power and utility. Ironically, the same language socialization ideologies and practices of accommodation, cooperation, and inclusion that once nurtured maintenance of the local village sign language now contribute to its decline and replacement by the national sign language.

\section{Acknowledgements}

At different times and in distinct ways, many people have helped bring this piece to fruition. I am grateful to Dr. Carol Padden, Dr. Owen Wrigley, and the Chao Din Student Organization at Mahasarakham University for granting permission to reproduce images from their respective publications and blogs. Thank you, Phaiwan, not only for your linguistic expertise, but also for your time and patience as a research consultant. Others who rendered kind assistance along the way include: Poonpit Amatyakul, Pimpa Kanchondham; Nipha Sukhwan, Vien Champa, the Madminggao family, Mi Kyung Kim, Merav 
Shohet, Chalita Mann, Kate Mesh, Tony Wright, Toni Comer, Robin Arora, Ulrike Zeshan, Connie de Vos, all of the EuroBABEL Village Sign project partners, and the community of Ban Khor. Generous financial support was provided by the National Science Foundation, the Wenner-Gren Foundation, the Endangered Language Fund, the Explorers Club, the University of Texas, the UCLA Department of Anthropology, the UCLA Wagatsuma Memorial Fund, the UCLA Center for Society and Genetics, UCLA Office of International Studies and Overseas Programs, IIE Fulbright, the Thai-US Educational Foundation, the Ratchasuda Foundation, and Ratchasuda College.

\section{Notes}

1. The spelling ecology(ies) is intentionally employed here to indicate the nuanced distinction between the broad similarities and shared characteristics (e.g., the singular 'ecology') among communities with village sign languages versus the ethnographic particulars and differences (e.g., the plural 'ecologies') between them.

2. Language ecology has been utilised in fields such as: sociology of language, sociolinguistics, dialectology, creole studies, language evolution, and so on.

3. In a seminal article entitled, "Language maintenance and language shift as a field of inquiry," Fishman (1964) notes that "social-psychological aspects of language" (or what today would be termed, 'use-based issues' like language socialization, ideologies, and practices) —impact language vitality or endangerment. He identifies ethnography (e.g., Ethnography of Speaking and Ethnography of Communication) as two possible approaches for investigating such matter (pgs. 64-65). For anthropologists, Sommer's attempt to prescriptively define ethnography in terms of his own particular research agenda is problematic, but his general analysis of the theoretical significance of anthropology and the methodological possibilities of ethnography for enhancing language ecology research is insightful.

4. For a summary of the history and activities of this research project and for an inventory of the project's anthropological and linguistic data corpora, see the sociolinguistic sketch of Ban Khor and Ban Khor Sign Language that appears elsewhere in this volume.

5. For more detailed information about Ban Khor's nucleated settlement pattern and for visual illustrations of it, see Nonaka 2009: 215-217.

6. In one case, the man is reported to have tortured a crow, even going so far as to cut off its beak. Thereafter, many of his descendants were born 'mute.' In the other case, it is said that a father, in a fit of anger, beat a small coconutgathering monkey to death, after which three of his children were suddenly born deaf. 
7. For additional information about the early development of Ban Khor Sign Language, see Nonaka 2007: 59-69.

8. Historically, and even today, the overwhelming majority of Ban Khorians were/are farmers. Within that social class there are detectable distinctions and hierarchies based on economic affluence, political prestige, and familial distinction. While such differences are real, they are relatively minor and can be fluid. Thus it is reasonable, for purposes of this discussion, to describe most farmers in Ban Khor as 'peers'.

9. The claim here is not that all villagers are fluent in BKSL, but rather that a significant portion of the village population was exposed to and developed some degree of competence in the local sign language. This claim is borne out both qualitatively through years of ethnographic observation and also quantitatively by social network analysis, the results of which were discussed earlier in Section 3.5.

10. For additional information about Thai deaf education see Branson et al. 2005, Reilly and Reilly 2005, Reilly and Suvannus 1999, and Suvannut 1987.

11. For a visual example, see the BKSL sign, FOREIGN, in line 1 of the transcript in Figure 1.

12. A longer, unpublished version of this transcript appears in (Nonaka 2007: 149-153). For those unfamiliar with standard CA transcription conventions, see Atkinson and Heritage 1984:ix-xvi.

13. The Frog Story is a popular elicitation device for international cross-linguistic research (Berman and Slobin 1994, 2002). The book consists entirely of a series of sequentially linked and topically coherent illustrations that tell a story (without words) about a little boy and his dog in search of a frog that has escaped from a glass jar. When used as a linguistic elicitation device, consultants are asked to look at the picture book and to narrate 'the'/a story in the target (typically their native) language. In their comparative research generated through The Frog Story, Berman and Slobin examine cross-linguistic development in narration and grammar with the goal of understanding linguistic universals, typological characteristics and languagespecific features of grammar as related to the recounting of narrative and by extension, cognition in context.

14. Photo: "Dai lai gwaa" (I have more than you do!) Excerpted from the Chao Din Student Organization's website, "Go to Know" Reprinted with permission of the authors.

15. Both the TSL and ASL signs LOVE are articulated on the chest with hands crossed (palm of the dominant hand atop the back side of the non-dominant hand) over the heart. They vary only in terms of handshape. The TSL sign is made with an extended thumb B-handshape, while the ASL sign I produced using a closed-fist S- handshape. 
16. Currently, there are approximately 400 registered interpreters in Thailand (Limanee 2012, personal correspondence). In Thailand, as in most countries, the history and development of sign language interpreting services is complicatedlinguistically, politically, and pragmatically. Many conversations over the years with members of the Thai Deaf community reveal that there is widespread agreement on the need not only for expansion of interpreting services but also for improvement of interpreters' signing proficiency. Improvement in both areas is occurring, although interpreters' 'fluency' in TSL remains a thorny issue. With regard to televised TSL interpretation, individual interpreters' TSL fluency levels vary, but even 15 years ago, at least three television interpreters were full-time interpreters at the National Association of the Deaf in Thailand.

17. An English-language translation of the Persons with Disabilities Empowerment Act B.E. 2550 is available online at the following website: http://www.nep. go.th/employment/doc/doc2.pdf.

\section{References}

Ash, A., J. L. D. Fermino \& K. Hale

2001 Diversity in local language maintenance and restoration: A reason for optimism. In L. Hinton and K. Hale, eds., The green book of language revitalization in practice. San Diego: Academic Press, 19-35.

Atkinson, J. Maxwell \& Heritage, J. (eds.)

1984 Structures of social action: Studies in conversational analysis. Cambridge: Cambridge University Press.

Augsburger, D.

2004 Language socialization and shift in an isthmus Zapotec community of Mexico. Doctoral dissertation, University of Pennsylvania.

Bahan, B. \& Poole-Nash, J.

1996 The formation of signing communities: Perspective from Martha's Vineyard. In J. Mann, ed., Deaf Studies IV Conference proceedings, 1-16. Washington, DC: Gallaudet University College of Continuing Education.

Berman, R. A. \& D. I. Slobin

1994 Relating events in narrative: A crosslinguistic developmental study. Hillsdale, NJ: Erlbaum.

Berman, R. A. \& D. I. Slobin

2002 Relating events in narrative: Variation across languages, cultures, and genres. Hillsdale, NJ: Lawrence Erlbaum Associates. 
Branson, J. \& D. Miller

1996 Everyone here speaks sign language, too: A deaf village in Bali, Indonesia-An initial report. In C. Lucas, ed., Multicultural aspects of sociolinguistics in deaf communities, 39-57. Washington, DC: Gallaudet University Press.

Branson, J., D. Miller, D. \& J. Sri-on

2005 A history of the education of deaf people in Thailand. Bangkok: Chulalongkorn University Printing House.

Chao Din Student Organization, Mahasarakham University.

2012 Dai lai gwaa (I have more than you do!), a photograph of a man "frogging" (hunting for frogs at night using the special fai song gop light worn on the forehead). Photo viewed on July 6, 2012 on the website: http://www.gotoknow.org/blogs/posts/272042 (website last updated on June 24, 2012).

Cumberbatch, $\mathrm{K}$.

2006 Country sign, St. Elizabeth, Jamaica. Presentation at the workshop on sign languages in village communities. Max Planck Institute for Psycholinguistics, Nijmegen, the Netherlands (April 4-6, 2006).

Dorian, N.

1981 Language death: The life cycle of a Scottish Gaelic dialect. Philadelphia: University of Pennsylvania Press.

Dorian, N. (ed.)

1989 Investigating obsolescence: Studies in language contraction and death. Cambridge: Cambridge University Press.

Enninger, W. \& L. M. Haynes (eds.)

$1984 \quad$ Studies in language ecology. Wiesbaden: Steiner.

Erting, C. J., C. Prezioso \& M. O'Grady-Hynes

1990 The interactional context of deaf mother-infant communication. In V. Volterra and C. J. Erting (eds.), From gesture to language in hearing and deaf children, 97-106. (Springer Series in Language and Communication 27.) New York: Springer..

Fader, A.

2006 Learning faith: Language socialization in a Hasidic community. Language in Society 35(2). 207-229.

Fader, A.

2007 Reclaiming sacred sparks: Linguistic syncretism and gendered language shift among Hasidic Jews in New York. Journal of Linguistic Anthropology 17 (1).1-22.

2009 Mitzvah girls: Bringing up the next generation of Hasidic Jews in Brooklyn. Princeton, NJ: Princeton University Press. 
Ferreiro-Brito, L.

1983 A comparative study of signs for time and space in Sao Paolo and Urubu-Kaapor Sign Language. In W. Stokoe and V. Volterra, eds., SLR 83. Proceedings of the $3^{\text {rd }}$ international symposium on sign language research, Rome, June 22-26, 1983. Silverspring, MD: Linstok Press, 22-26.

Field, M.

1997 Maintenance of ways of speaking in a community undergoing language shift: Language socialization in a Navajo preschool. Doctoral dissertation, University of California, Santa Barbara.

Fill, A. \& P. Mühlhäusler (eds.)

2001 The ecolinguistics reader: Language, ecology, and environment. London and New York: Continuum.

Fishman, J.A.

1964 Language maintenance and language shift as a field of inquiry. Linguistics 2 (9). 32-70.

Fox Tree, E.

2009 Meemul Tziij: An indigenous sign language complex of Mesoamerica. Sign language studies 9 (3). 324-58.

Frishberg, N.

1987 Ghanaian Sign Language. In J. V. Van Cleve, Gallaudet encyclopedia of deaf people and deafness. New York: McGrawHill, 778-779.

Gal, S.

1979 Language shift: social determinants of linguistic change in bilingual Austria. New York: Academic Press.

Garrett, P.

1990 Language socialization, convergence, and shift in St. Lucia, West Indies. Doctoral Dissertation, New York University.

Garrett, P.

2005 What a language is good for: Language socialization, language shift, and the persistence of code-specific genres in St. Lucia. Language in Society 34. 327-361.

Garrett, P.

2006 Contact languages as "endangered" languages: What is there to lose? Journal of Pidgin and Creole Languages 21 (1). 175-190.

Grenoble, L.

2011 Language ecology and endangerment. In P.K. Austin and J. Sallabank, eds., The Cambridge handbook of endangered languages, 27-44. Cambridge: Cambridge University Press. 
Groce, N. E.

1985 Everyone here spoke sign language: Hereditary deafness in Martha's Vineyard. Cambridge, MA: Harvard University Press.

Hand, W. D.

1980 Deformity, disease and physical ailment as divine retribution. In W. D. Hand ed., Magical medicine, 57-67. Berkeley: University of California Press.

Hanks, J. R.

1963 Maternity and its rituals in Bang Chan. Cornell University Southeast Asia Program, data paper 51. Ithaca, NY: Cornell University.

Hanks, L. M.

1962 Merit and power in the Thai social order. American anthropologist 64. 1247-1261.

Harrison, K. D., D. S. Rood \& A. Dwyer

2008 A world of many voices: editors' introduction. In K.D. Harrison, D.S. Rood, and A. Dwyer eds., Lessons from documented endangered languages, 1-11.. Amsterdam and Philadelphia: John Benjamins Publishing Company

Haugen, E.

1972 The ecology of language. Stanford, CA: Stanford University Press. Hoffman, K.E.

2007 We share walls: Language, land, and gender in Berber, Morocco. Malden, MA: Blackwell Publishing.

Howard, K. M.

2003 Language socialization in a northern Thai bilingual community. Doctoral dissertation. Los Angeles, CA: University of California, Los Angeles.

Howard, K. M.

2004 Socializing respect at school in northern Thailand. Working Papers in Educational Linguistics 20 (1). 1-30.

Humphries, Tom; Padden, Carol; and O'Rourke, Terrence J.

1985 A Basic Course in American Sign Language (12 $2^{\text {th }}$ printing). Silver Spring, MD: TJ Publishers.

Hymes, D.H.

1962 The ethnography of speaking. In T. Gladwin and W. C. Sturtevand (eds.), Anthropology and human behavior. Washington, D.C., Anthropology Society of Washington, 13-53. 
Ingersoll, J.

1975 Merit and identity. In G. W. Skinner and A. T. Kirsch (eds.), Change and persistence in Thai society: Essays in honor of Lauriston Sharp, 219-273. Ithaca, NY: Cornell University Press.

Johnson, R. E.

1991

Johnson, R. E.

Sign language, culture, and community in a Yucatec-Mayan village. Sign language studies 73. 461-74.

1994 Sign language and the concept of deafness in a traditional Yucatec Mayan Village. In C. J. Erting et al., eds., The deaf way: Perspectives from the international conference on deaf culture, 103-109. Washington, DC: Gallaudet University Press.

Jones, M.C.

1998 Language obsolescence and revitalization: Linguistic change in two sociolinguistically contrasting communities. Oxford: Oxford University Press.

Kakumasu, J.

1968 Urubu Sign Language. International journal of American linguistics 34. 275-281.

Keyes, C. F.

1983a Introduction: The study of popular ideas of karma. In C. F. Keyes and E. D. Valentine eds., Karma: An anthropological inquiry, 1-24. Berkeley, CA: University of California Press.

Keyes, C. F.

1983b Merit transference in the kammic theory of popular Theravada Buddhism. In C. F. Keyes and E. D. Valentine eds., Karma: An anthropological inquiry. Berkeley, CA: University of California Press, 261-286.

Keyes, C. F.

1995 The golden peninsula: Culture and adaptation in mainland southeast Asia. Honolulu: University of Hawaii Press.

Kirsch, A. T.

1977 Complexity in the Thai religious system: An interpretation. The journal of Asian studies 36 (2). 241-266.

Kisch, S.

2004 Negotiating (genetic) deafness in a Bedouin community. In J.V. Van Cleve ed., Genetics, disability, and deafness, 148-173. Washington, DC: Gallaudet University Press.

2008 The social construction of deafness in a Bedouin community in the Negev . Medical Anthropology 27(3). 283-313. 
Kulick, D.

1992 Language shift and cultural reproduction: Socialization, self, and syncretism in a Papua New Guinean village. Cambridge: Cambridge University Press.

Kuschel, R.

1973 The silent inventor: The creation of a sign language by the only deaf-mute on a Polynesian island. Sign language studies 2 (3). 1-28.

Ladd, $\mathrm{P}$.

2003 Understanding Deaf Culture: in Search of Deafhood.Clevedeon, England: Multilingual Matters.

Lane, $\mathrm{H}$.

1984 When The Mind Hears. New York: Random House.

Leonard, W.Y.

2007 Miami Language Reclamation in the Home: A Case Study. Doctoral dissertation, University of California, Berkeley.

Leonard, W.Y.

2008 When is an 'extinct language' not extinct? Miami, a formerly sleeping language. In K. King, A. Schilling-Estes, N. Fogle, L. Lou, J. Jia, and B. Soukup, eds. Sustaining linguistic diversity: Endangered and minority languages and language varieties, 23 33. Washington, DC: Georgetown University Press.

Marsaja, I. G.

2008 Desa kolok: A deaf village and its sign language in Bali, Indonesia. Nijmegen, The Netherlands: Ishara Press.

Mayer, Mercer

1980 Frog, where are you? New York: Dial Press.

Meek, B.A.

2001 Kaska language socialization, acquisition, and shift. Doctoral dissertation, University of Arizona.

Meek, B.A.

2007 "Respecting the Language of Elders: Ideological Shift and Linguistic Discontinuity in a Northern Athapascan Community." Journal of Linguistic Anthropology 17 (1). 23-43.

Mizuno, K.

1971 Social system of Don Daeng village-A community study in northeast Thailand. The Center for Southeast Asian Studies. Ithaca, NY: Cornell University. 
Mühlhäusler, P.

1997 Language ecology_contact without conflict. In M. Pütz (ed.).

Language choices: Conditions, constraints, and consequences, 3-15. Amsterdam and Philadelphia: John Benjamins Publishing Company.

Nonaka, A. M.

2004 The forgotten endangered languages: Lessons on the importance of remembering from Thailand's Ban Khor sign language. Language in society 33. 737-67.

Nonaka, A. M.

2007 Emergence of an indigenous sign language and a speech/sign community in Ban Khor, Thailand. Doctoral dissertation. Los Angeles, CA: University of California, Los Angeles.

Nonaka, A. M.

2009 Estimating size, scope, and membership of the speech/sign communities of undocumented indigenous/village sign languages: The Ban Khor case study. Language and communication 29. 210 229 .

Nonaka, A. M.

2011 Language socialization and language endangerment. In Duranti, A., E. Ochs, \& B.B. Schifflin, (eds.), The handbook of language socialization.Wiley-Blackwell. 610-630.

Nyst, V.

2007 A descriptive analysis of Adamorobe Sign Language (Ghana). LOT dissertation series 151. Netherlands Graduate School of Linguistics.

Ochs, E. and Schieffelin, B. (eds.)

1984 Language acquisition and socialization: Three developmental stories and their implications. In R. A. Schweder and R.A. Levine eds., Culture theory: Essays on Mind, Self, and Emotion. New York: Cambridge University Press, 276-320.

Pappu, S. S. Rama Rao (ed.)

1987 The dimensions of karma. Delhi: Chanakya Publications.

Paugh, A.L.

2001 "Creole day is every day": Language socialization, shift, and ideologies in Dominica, West Indies. Doctoral Dissertation, New York University.

Phillips, S.

1983 The invisible culture: Communication in classroom and community on the Warm Springs Indiana Reservation. New York: Longman. 
Piker, S.

1964 An examination of character and socialization in a Thai peasant community. Doctoral dissertation. Washington, DC: University of Washington.

Prasad, R.

1989 Karma, causation and retributive morality: Conceptual essays in ethics and metaethics. Indian Council of Philosophical Research Series in Contemporary Indian Philosophy. New Delhi: Munshira Manoharlal Publishers.

Reagan, T.

1995 A Sociocultural Understanding of Deafness: American Sign Language and the Culture of Deaf people. International Journal of Intercultural Relations, 19(2). 239-252.

Reardon, W., H. Toriello \& C. Downs

2004 Chapter 3: Epidemiology, etiology, genetic patterns, and genetic counseling. In H. Toriello, W. Reardon, and R. Gorlin eds., Hereditary hearing loss and its syndromes, 8-16. Oxford: Oxford University Press.

Reilly, C. \& N. Reilly

2005 The rising of lotus flowers: Self-education by deaf children in Thai boarding schools. Washington, DC: Gallaudet University Press.

Reilly, C. \& S. Suvannus

1999 Education of deaf people in the kingdom of Thailand. In H.W. Brelje (ed.) Global perspectives on education of the deaf in selected countries, 367-382. Hillsboro, OR: Butte.

Roeder, E. W.

2001 Status of educational resources and facilities for the physically and intellectually disabled in Thailand. Unpublished M.A. thesis, University of Hawaii.

Royal Thai Government.

2550 [2007] English-language translation of the Persons with Disabilities Empowerment Act B.E. 2550. http://www.nep.go.th/employment/ doc/doc2.pdf.

Sandler, W., I. Meir, C. Padden \& M. Aronoff

2005 The emergence of grammar: Systematic structure in a new language. Proceedings of the National Academy of Sciences 102 (7). 2661-2665. 
Shuman, M. K.

1980 The sound of silence: A preliminary account of sign language use by the deaf in a Maya community in Yucatan, Mexico. Language sciences 2 (1). 144-173.

Smalley, W. A.

1994 Linguistic diversity and national unity: Language ecology in Thailand. Chicago: University of Chicago Press.

Sommer, G.S.

1997 Towards an ethnography of language shift: Goals and methods. In M. Pütz (ed.) Language choices: Conditions, constraints, and consequences, 55-76. Philadelphia, PA: John Benjamins Publishing Company.

Suvannus, S.

1987 Thailand. In J. Van Cleve (ed), The Gallaudet encyclopedia of deaf people and deafness, 282-84. New York: McGraw-Hill.

Suwanarat, M., A. Ratanasint, V. Rungsrithong, L. Anderson \& O. P. Wrigley

1990 The Thai Sign Language dictionary (revised and expanded edition). Bangkok: National Association of the Deaf in Thailand.

Torigoe, T., W. Takei \& H. Kimura

1995 Deaf life on isolated Japanese islands. Sign language studies 24 (87). 167-174.

Uchikawa, T.

1991 Conquest of cause and effect retribution argument. In World Federation of the Deaf (WFD). Equality and self-reliance: Proceedings of the XI world congress of the world federation of the deaf, 905-915. Tokyo: Japanese Federation of the Deaf.

Van den Bogaerde, B.

2006 Kajana Sign Language, Surinam. Presentation at the workshop on sign languages in village communities. Max Planck Institute for Psycholinguistics, Nijmegen, the Netherlands (April 4-6, 2006).

Washabaugh, W.

1978 Providence Island Sign Language: A context-dependent language. Anthropological linguistics 20 (3). 95-109.

Washabaugh, W.

1979 The deaf of Grand Cayman, British West Indies. Sign language studies 31. 117-133.

Washabaugh, W.

$1981 \quad$ Hearing and deaf signers on Providence Island. Sign language studies 24. 191-214. 
Washabaugh, W.

1986 Five fingers for survival: Sign language in the Caribbean. Ann Arbor, MI: Karoma Press.

Weiss, M. G.

1980 Caraka Samhita on the doctrine of karma. In W. D. O'Flaherty ed., Karma and rebirth in classical Indian traditions. Berkeley, CA: University of California Press, 90-115.

Woodward, J. C.

1975 How you gonna get to heaven if you can't talk with Jesus: The educational establishment vs. the Deaf community. Revised and published as Woodward 1982.

Woodward, J. C.

1982 Beliefs about and attitudes towards deaf people and sign language on Providence Island. In J. C. Woodward ed., How you gonna get to heaven if you can't talk with Jesus: On depathologizing deafness., 51-74 Silver Spring, MD: T. J. Publishers.

Woodward, J. C.

1996 Modern Standard Thai Sign Language, influence from ASL, and its relationship to original Thai sign language varieties. Sign Language Studies 92. 227-252.

Woodward, J. C.

2000 Sign languages and sign language families in Thailand and Vietnam. In K. Emmorey and H. Lane eds., The signs of language revisited. Mahwah, NJ: Lawrence Erlbaum, 23-47.

Zeshan, U.

2004 Interrogative constructions in signed languages: Cross-linguistic perspectives. Language 80 (1). 7-39.

Zeshan, U. (ed.)

2006 Interrogative and negative constructions in sign languages. Nijmegen, The Netherlands: Ishara Press. 


\title{
Working with village sign language communities: Deaf fieldwork researchers in professional dialogue
}

\author{
Hasan Dikyuva, Cesar Ernesto Escobedo Delgado, \\ Sibaji Panda and Ulrike Zeshan
}

\section{Introduction ${ }^{1}$}

In this chapter, we report on work in three different communities with a high incidence of hereditary deafness and indigenous sign languages. These are the villages of Alipur in South India and Chican in Mexico's Yucatan Peninsula, as well as the community of Mardin Sign Language users in Turkey. Alipur and Chican, with 150 deaf people out of 20,000 and 17 deaf people out of 700 respectively, are similar in sociolinguistic set-up to the betterdocumented "deaf villages" in Bali (Marsaja 2008; de Vos 2012) and Ghana (Nyst 2007). Mardin Sign Language, which originated in the town of Mardin in south-eastern Turkey, is now on the brink of extinction and used by ca. 40 deaf and hearing members of a single extended family, all of whom have moved to the metropolitan areas of Istanbul and Izmir. In all three cases, the sign language has been in existence for several generations. Details about each of these communities can be found in the sociolinguistic sketches in this volume (contributions by Panda, Escobedo Delgado, and Dikyuva).

The intention of this chapter is to add to the emerging discussion in sign language linguistics on issues of ethical practice and community engagement during fieldwork. In particular, the approach is to represent the experiences of deaf fieldwork researchers working with rural and/or small-scale sign language communities in their countries of origin. To this effect, in May 2012 three of the authors (Sibaji Panda (SP) from India, Cesar Ernesto Escobedo Delgado (CEED) from Mexico and Hasan Dikyuva (HD) from Turkey) engaged in academic dialogues about the same set of questions. The dialogues were conducted in International Sign and video recorded. The transcripts were then translated into English. These data, comprising 2.5 hours of video and over 21,000 words in English, are used in the present chapter. 
Personal accounts of linguistic fieldwork by researchers are now reasonably common for spoken languages, in particular in the area of language documentation and endangerment. For instance, Wilkins (1992) relates his experiences with work on Aboriginal languages in Central Australia, and Debenport (2010) discusses her work with a Tiwa-speaking Pueblo community in New Mexico. In contrast, similar personal accounts by sign language researchers, such as in Jones \& Pullen (1992), are still relatively rare. Although the field of sign language linguistics has always been very concerned with ethics, and ethical issues are now more often discussed explicitly (e.g. Harris, Holmes \& Mertens 2009), it is not easy to locate authentic first-hand accounts of fieldwork practice in the sign language literature. In particular, the "voices" of deaf researchers and researchers from developing countries are largely absent from public discourse. ${ }^{2}$ Therefore, our aim is to consider issues of fieldwork practice from the perspective of deaf researchers working in their home countries.

Most of the work that we relate in this chapter is taking place as part of a project on "endangered village sign languages" funded by the UK's Arts and Humanities Research Council (AHRC) through the EuroBABEL stream of the European Science Foundation's EUROCORES scheme. ${ }^{3}$ This is a large consortium project conducted by partner universities in five countries, and the authors belong to the UK research group, which is the lead partner. In addition, work in Alipur and on Mardin Sign Language has also been supported by grants from the Endangered Languages Documentation Programme (ELDP). The "applied" community projects we report on include the opening of a school for the deaf in Alipur, the creation of multimedia educational materials on Mardin Sign Language, and the compilation of a dictionary of Chican Sign Language.

In quoting from the academic dialogue data, while we do not always comment on research ethics in explicit, formal, or theoretical terms, it is evident in our work that we are deeply concerned with many issues that are relevant to theoretical discussions and methodological developments in the field. In Sections 2-6, we consider several pertinent issues and scenarios as they play out in each of the three field sites, using lengthy direct quotes from the professional dialogues. Work in each of the three communities constitutes a detailed case study, and Section 7 reflects on the importance of such material and draws conclusions of a general nature. 


\section{Preparatory phase: Discovering the language and the community}

The extracts from the video material in this section describe the initial contact between the deaf EuroBABEL fieldwork researchers and the respective communities. A considerable level of personal interest and increasing involvement is evident from these comments. SP and CEED discuss the first contact with the field site as follows:

SP: I'd like to ask you, about your research location of Chican, what was your first contact with the village, or how did you find it in the first place? How did you contact them?

CEED: I first saw it on the web. There was some information from America about Mexico and Chican Sign Language, calling it Yucatec Mayan Sign Language. I read this and I was confused, like we have our LSM (that is, Lengua de Señas Mexicana, or Mexican Sign Language), but the sign language in that village was supposed to be different, but why and how? That caught my attention and I wanted to have a look and took a flight to go there. ${ }^{4}$ I had a deaf friend from Merida, which is in Yucatan, so he took me there. We entered and there were all the deaf in the village, and it was different. Their culture was different, their clothes were different, their food, houses, everything was different. It was very interesting. I met the people for the first time and signed with them, and I was very interested and really surprised. I was intrigued and wanted to know more about their situation. I found that my own Mexican signs and their signs were different.

SP: In my case, this is how the first contact happened: In 2006 I was working in the Netherlands, and world-famous people came there for a workshop uniquely on village sign languages. We discussed, and I saw the lectures, and I was impressed and interested. I thought that probably India does have several such villages. So I did a lot of searching on the web, and I found a newspaper article. I read it, and yes, there was such a village, with about 200 people with disabilities, blind, deaf, various. This made interesting reading and I kept a copy, though initially, I did not have any funding. I took a flight there on my own initiative, to South India, Bangalore. I didn't know where to go from there, and I searched around for two days. I would go the wrong way, stay for the night, again come back, and nobody knew clearly where it was. After roaming around for a while, I finally found it. I had started out in the morning, and towards the evening, around four o'clock, I was there and had found it, after a long time searching. It was like a shock at first contact, that I had really found the right thing.... The signs were different, and the place was full of deaf people signing, children, adults, all signing. ... The village has its own particular sign language, and all the criteria from the previous lectures in the Netherlands about village sign languages matched this situation, like testing positively for these criteria. 
CEED: So you were searching around and going the wrong way etc for two days. I got there in one day, because luckily, the Mexican deaf association helped me. They gave me instructions how to change buses twice, starting from the main road, and into ever smaller roads, across hills, around bends etc, then the village was there. There are about 700 hearing people, and 18 deaf, but one has died, so now there are 17 deaf people. So the way I contacted and met these people was through the president of the deaf association, who knew an interpreter who was working with the police .... So we found him and talked to him, the two of us deaf (CEED and the local deaf association president) signing LSM, we were wondering what to do. We said, where are the (local) deaf, and he (the interpreter) took us to their homes. We said hello and took some photos and talked in sign language. We went to all the various houses and in the evening we all gathered together; we freshened up and dressed up well and we gathered and started signing and working together. We established a relationship slowly, not feeling quite sure of ourselves first.

It is evident from both these accounts that the prospect of visiting a "deaf village" has been intriguing and exciting. Although travel to the field sites involved considerable personal effort and expense, there is a palpable sense of emotional involvement and curiosity throughout this process of discovery. CEED is using his existing network across Mexico, as he was president of the National Union of the Deaf at the time, while SP would have found it much easier than a foreign visitor to navigate rural India, as he himself grew up in a village in a different part of India.

Another interesting point is that neither CEED nor SP first entered the village with the aim of conducting research, but rather visited out of a personal curiosity. They comment on how they first started to think of assisting the local community by doing research (SP) and creating sign language resources (CEED), after having spent some time in the village already:

SP: I didn't show them anything about doing research at first. I just met them as a deaf person, in a visual way, through pictures, just like them. I just went and greeted them and met with them, that was all. I didn't really have a firm research plan in the back of my mind at that time, I didn't know... I felt quite happy, because from the previous lectures in the Netherlands I really was very interested in finding such a situation, and when I arrived there in reality for the first time and it matched, I felt good. It was a new experience. So I started to think how I would do research there later, how to help the locals, and I started planning.

CEED: I wanted to give my support for releasing a dictionary of the local Chican Sign Language. So I was thinking that I did not have any resources myself. I thought I would save up some money for later work, or contact some others for fundraising, then I would fly there again and make something happen. There was no funding at the time, so I dropped it for the time being. 
In the case of Mardin Sign Language, a different kind of coincidence led to the discovery of its community, and HD relates the situation as follows:

HD: In 2004, I was working with some students, providing sign language teacher training for them. I had assembled these trainees to teach them about language and linguistics, so they could understand how (to teach). The students all became more aware of their own language, and one of them realised that she had another sign language (in her background), from Mardin. She started telling me that there is this other sign language from the village, and it is important. This person was important, and we met and discussed how something could be done. I met with several other people from the family, and their signs were completely different. The sign language used in (other parts of) southeast Turkey and the Mardin Sign Language were completely different. When I met these people, I realised that this sign language is important.

The discovery of Mardin Sign Language was facilitated by HD's prominent position as the first trainer for teachers of Turkish Sign Language. This case is particularly intriguing in that the users of Mardin Sign Language had been living among the Turkish Sign Language community for decades without anyone becoming aware of the status or existence of their minority sign language. This was only possible through the training programme and the enhanced meta-linguistic skills it provided. Here again, the motivation for research developed gradually over time:

HD: For the EuroBABEL project, I do fieldwork with the (people from) Mardin. This was really not easy, it was difficult, but this student of mine helped me. We went together, as this is her own family. I met several of them, and I could not understand their signing at all. I did not immediately begin with research. That was not my aim. I just met the people, and when I could not understand them, I spent time learning their signs.

A general point emerges from these observations regarding the importance of taking a long-term perspective. The initial familiarisation and engagement with the communities took a long time, and certainly much longer than the official project duration would have allowed. This means that the requirements of a typical scientific project are at odds with the requirements of effective community engagement in terms of the necessary time frame. The initial contact with the communities goes back several years before the beginning of the project, to 2004, 2005 and 2007 respectively, and the subsequent community projects described in Section 4 build on this longstanding contact. It is clear in retrospect that such activities would not have been feasible within the time limit of a typical research project because of insufficient "lead-up time".

In the case of urban sign languages, consistent long-term community engagement is typically much easier to achieve because researchers often 
live and work in close proximity to the Deaf community. However, the situation of fieldwork in a less accessible village such as Chican or Alipur is much more difficult and more closely comparable to fieldwork on endangered spoken languages in rural, sometimes remote areas. Moreover, the time lag before starting a formal project and the time spent on follow-up activities after completion are all the more valuable in cases where the target communities have little or no previous experience with linguistic research (see the discussion in Section 3).

Ideally, we would like to think of language documentation as an openended collaborative activity rather than a time-limited project. In some cases of research on rural sign languages and their communities, such as in Kisch (this volume) and Nonaka (this volume), contact with a community spans over a decade or more. Working over such long time scales is a significant challenge, given that most funded academic projects only run for two or three years, but mutual respect and trust, the core ingredients of any successful community project, build up very gradually over time.

\section{Start-up phase: The beginnings of research activities}

From the beginning, the EuroBABEL project was designed to engage with the target communities and to consider substantial local benefit, including the provision of a separate budget for such activities. However, the means of achieving this are far from straightforward, and the observations about the challenges of starting research activities in this section as well as the subsequent decision-making processes (Section 4) are instructive in this regard. This section considers the specific backgrounds of our target communities with respect to people's previous experience with researchers and other professionals (3.1), as well as the impact this has on the notion of informed consent (3.2).

\subsection{Considering the "research history" of field sites}

In order to understand the challenges in starting research activities, we first need to consider the background situation at the three field sites with respect to previous experiences with research, technology, and language documentation. On the one hand, linguistic research was very much a novelty in these communities, but on the other hand, some of them did have experience with visits by external professionals or researchers from other academic disci- 
plines. Such experiences, or the lack thereof, may have influenced their initial reaction to the deaf researchers, as SP and CEED illustrate:

SP: My first contact (with the village) was quite interesting, because I had a friend from Belgium with me and we both went together. We are both deaf, and the people in the village had never met a white deaf person from outside. They had never seen a video camera for filming. They were very interested and flocked towards us readily. So actually for the first contact, I didn't know anything about their homes, about the village, the research. I just arrived and met the deaf for about two hours only, because it was already getting late in the evening and there were no electric lights outside. ...They themselves were also equally intrigued to see a white person coming. That had an impact on them. Later when I kept going there, they always asked me where my white friend was.

CEED: In my case, I was not the first one. There had been people before, Americans, French, Canadian, a number of them. I was the first deaf person though. ... I promised to make a dictionary of their sign language and give it to them, so they were happy.

SP: When I arrived for the first time, this is what people told me: the village had been visited by audiologists, doctors, geneticists, who had taken blood samples for medical research, and this had happened repeatedly. There had not been any linguists or sign language researchers ever visiting there before ... Nobody ever came to collect video data. I was the first one. ... There was no previous standard to evaluate me against. The only thing they especially perceived and remembered about me was that I was deaf, and we felt very compatible.

CEED: Similarly in Chican, there were more doctors, more geneticists, like in your case. People had been distributing hearing aids, cochlear implants, but giving them something about the sign language, education and schooling, that had not been done. There was only concern about hearing aids and such. I saw that this was of no benefit whatsoever, and people were saying, whatever, they gave us these things, so what? People would wear them for the photos, for good PR in the newspapers and some applause for show. I had doubts about that, and I wanted to make an impact for the sign language, to spread and respect their sign language.

Thus both in Alipur and in Chican, we were not the first to visit and to be interested in the situation regarding deafness. Medical doctors, audiologists, geneticists and, in the case of Chican, anthropologists had already visited these villages, and the resulting experiences were not always satisfactory for the locals. This is a pertinent point, as people do tend to draw comparisons with other visitors, which can cause tensions (cf. the chapter on Adamorobe in Ghana by Kusters, this volume). In Alipur, the presence of a white 
foreigner, video cameras, language documentation materials, computers and the Internet, and indeed the whole concept of research, was alien to the local deaf people. ${ }^{5}$ In both cases though, the appearance of deaf researchers made a particular impact on the local people. This issue is discussed in more detail in Section 6.

For Mardin Sign Language, the situation was different as we are working with a community effectively in diaspora in Istanbul and Izmir. Therefore, they have ready access to and experience with technology such as video cameras and the Internet. However, as research on Mardin Sign Language had never been conducted, HD also reports that there was a long initial phase during which the Mardin Sign Language users had to become acquainted with language documentation in order to accept the importance of this work for their unique language:

HD: You meet someone, and the person thinks, what is this research, what is the aim. They thought it is not important (to them), and that it should not be important to me either. Then I needed to engage with them (and explain why) it is important. They said that Turkish Sign Language is already used everywhere, and the general attitude and expectation is that Mardin Sign Language is of much lower status. ... For me as a fieldworker, in my view the language of the people in this community is important. However, they had no concept that passing on their language to the next generations was important. Mardin Sign Language was just an okay kind of thing. They did not have the awareness yet. As time passed, I kept giving them information, we had workshops, and they themselves became aware that this is important. They themselves understood that Mardin Sign Language is our own language and is important to us. Initially, they didn't understand that their signs are a linguistic heritage of their own to be carried forward and are important to be preserved. A lot of time was needed for repeated workshops to generate (these ideas).

In Section 4, where decision-making for implementing community projects is discussed, such processes of changing attitudes are discussed in more detail within the particular field settings.

\subsection{Informed consent}

Considering the previous "research history" in a community is not only vital for helping researchers avoid rushing into something that may have negative connotations for the participants. These considerations also have a more direct, practical impact on the issue of informed consent, that is, the consent that participants capable of autonomous decisions give voluntarily to researchers after comprehensive briefing about aims, procedures, 
and possible risks and benefits of their participation. Since informed consent originates from medical research, in principle it should be obtained at the beginning of a project, and certainly before any data collection activities. Informed consent is a central notion in all professional codes of ethics or ethical guidelines, including those of linguists (e.g. LSA 2009).

However, this principle can be a challenge when working with Deaf communities, and certainly in the kinds of communities that our project was operating in. These challenges are not often made explicit, as considering them may force researchers to make uncomfortable decisions. An interesting example is given in Crasborn (2010), who comments on work with users of Sign Language of the Netherlands (NGT) for a corpus project involving the open-access publication of video files on the Internet:

Although the statements on the consent form may be explained to them in sign language (...), this does not count as a voluntary and well-informed decision if people are not fully literate and cannot comprehend the impact of a short, written document like a consent form. Second, ... do people understand what publication on the Internet means? ... If one has no experience browsing web pages from all over the world ..., it is hard to decide whether or not video recordings of oneself should be made available to the whole world.

(Crasborn 2010:285-86)

In this case, the research team relied on their personal judgements to decide that genuine informed consent was indeed possible, concluding that "it was the impression of our Deaf assistant that even the older signers in the Corpus NGT were acquainted with the Internet and would be able to comprehend the meaning of "worldwide availability" (ibid: 286).

As should be clear from the comments in Section 3.1, the situation with the signers from Chican, Alipur, and Mardin is far more difficult to tackle. In all cases and particularly for the latter two communities, it is very obvious that given the lack of relevant background knowledge on more than one level, genuine informed consent was an impossible notion at the beginning of research activities. In fact, it is our collective experience that informed consent based on a sufficiently sophisticated understanding of research has been a result rather than a precondition of the research process, and we discuss the implications of this realisation below.

It is interesting that despite working independently of each other, a similar approach was used in all three communities to navigate around this thorny issue. This involved a strategy of slowly feeling their way forward at the beginning of their research, that is, after the initial get-to-know phase was over and they wanted to proceed with actual research. 
HD: I tried to explain about research, but people there could not understand. There was something like a disagreement, which was a bit difficult for me. So I just forgot about trying to explain, and just asked them whether I could film them. They agreed readily. They have (family functions) where they come together, like weddings, or for circumcision, which is a Muslim custom. They accepted to be filmed on those occasions. So I did a lot of filming, but people were wondering why I was not filming the party and was filming individual deaf people using sign language instead. ... I tried to explain why filming and collecting the sign language was important, but I felt that they did not get a clear idea at all. I think they had never had any similar experience before where such video recordings were made. I still tried to get a good collection of videos.

HD started filming in a setting where according to the local culture, this was already considered natural, although for quite different purposes. Although at this stage none of the participants had grasped the concept of linguistic research and therefore, obtaining genuine informed consent was impossible, HD's personal judgement was that filming in this setting would be neither intrusive nor harmful. SP's experiences are similar in this respect:

SP: When I arrived for the first time for fieldwork, I did not start filming immediately. I first looked around and shook hands with people, and all should understand first who I was and what I had come for. ... They all had no idea what research meant, and I also didn't rush into explaining it to them. I first met with them and talked about other things, like the importance of studying for them, knowing how to write. They were facing barriers; for example, they were not able to send SMS messages by mobile phone. All the hearing people could do that, but they couldn't. So I explained such things to them, and I organised several workshops within one week .... What I filmed was nothing in particular, only free communication that was happening anyway, which I filmed by observing in the background.

It is evident here that SP primarily considers it important to engage with the situation of deaf signers in Alipur, including their status as a disadvantaged group, whereas obtaining video data is happening in the background, almost in a casual manner. Similarly, CEED describes the initial process of building a rapport with deaf signers in Chican, while also commenting on his own difficulties.

CEED: I was quite nervous and shaky and felt uncertain, because I was doing this for the first time. I didn't really have experience in this job, in research, collecting video data and all. I had never done that. It was the first time for me. But I got some training, and UZ had the experience, and I was really up for it and interested. So I arrived back in Mexico by plane. I hired a car and paid for the petrol and the car hire, and the driver left, and I was there. I 
was introduced to people, and I slowly started signing with them. There was tentative eye contact, and I did not start filming yet, I held back. So slowly we were signing, eating together the food I provided, and they started to be open and share things. It was a bit of work to loosen them up, and we started to gel with each other. They were intrigued that I was also deaf like them, and signing and gesturing with them. I started filming their signs, like the numbers 100,200 , and stories. I would encourage them and they expressed themselves and I collected the signing. ... I showed them that I was using LSM with my other deaf friend, and they were surprised to see how different it was. Equally, we saw that their signs were different; I already knew that. There was interpreting and translation going on between the two versions of signing, and we looked at each other's signs and were intrigued. I respected their signs, and they respected mine, and understood that they had their own sign language. I kept teaching them that the urban areas and Mexico City are different, and the village here has its own sign language. ${ }^{6}$ So that opened their minds, and slowly over time, we started to match well with each other.

In the professional dialogue, the notion of respect is discussed frequently by CEED, and clearly, this is considered more important than a formal requirement such as obtaining a consent form. Moreover, it is evident in all three accounts that the initial steps taken are very small, while the responses from participants are constantly being evaluated. This is to try to get a sense of the participants' priorities, and to ascertain what level of understanding the participants may be developing about research on their languages.

This way of talking about first steps at the beginning of research activities is quite similar to what Kubanyiova (2008) discusses in terms of conflicts between "macroethics" and "microethics". Discussing a project in Applied Linguistics, she maintains that "when research becomes highly situated, it is as if suddenly a can of ethical worms is opened, and what seemed straightforward and logical at the macro-level suddenly becomes ambiguous and problematic in the actual research practice, rendering existing ethical guidelines inadequate" (Kubanyiova 2008:515). The macro-level consists of general principles laid down, for example, in professional codes of ethics, while the level of microethics is concerned with the particular requirements of a very specific situation, which may be in conflict with macroethical principles. In our case of informed consent from users of village sign languages prior to data collection, it would certainly have been possible to give explanations through the local sign language and obtain their agreement in some formal way - after all, no objections to video filming were raised in any of the three communities. ${ }^{7}$ However, unless one is satisfied with a rubberstamping exercise, such consent is largely meaningless if the participants have no background knowledge of linguistic research, and realistically, they 
can only acquire this understanding during the course of research itself. This concerns not only informed consent but also a whole range of other issues such as anonymity, data protection, confidentiality, and acknowledgements, and publication media.

Faced with this conundrum, we used our personal judgement to slowly feel our way forward, in the ways described above. This level of personal responsibility, and indeed personal risk, is increasingly being recognised as researchers now frequently work in multi- and cross- cultural contexts. This requires quite a different set of skills based on the concept of research ethics as a continuous process rather than a set of pre-conditions to research. Jones \& Pullen (1992:196) refer to the long-term "moral career" of researchers in their discussion of tensions between "Deaf and hearing worlds". This is similar to the notion of "virtue ethics", which "does not place emphasis on following principles but rather on the development of the moral character of the researcher, his/her ability and willingness to discern situations with potential ethical ramifications as they arise in the research practice, and his/ her ability to make decisions", so that formal ethics requirements are balanced with situationally adequate behaviour (Kubanyiova 2008:507). Although no such theoretical notions are mentioned in the professional dialogues, this is effectively our approach. The following quotes describe how consent was eventually obtained at times and in ways judged appropriate by the research team. In Chican, people were already familiar with researchers, so the main issue was the way in which information was being communicated:

CEED: There was an existing consent form from the university in Preston. I had the form translated into sign language by an interpreter and filmed that, because people there are illiterate and don't read Spanish. I first translated the form from English into Spanish, and when I showed it to them, they didn't understand, so I called an interpreter, who translated it into Chican Sign Language, like, did they agree for their sign language data to be made public? This was explained in sign language and filmed, and when it was clear, they signed on the forms. It was good. The only problem was that there was one child, 12 years old, so I asked the father to come. I gave explanations to him, and asked if there wouldn't be any problem, that I wouldn't be blamed afterwards. But that was not the case, and eventually, everything was signed and it was fine.

By contrast, SP followed a very different approach, which was motivated by the particular hierarchical organisation in the administration of Alipur:

SP: For me, it was different. I first proceeded slowly with some filming, and I didn't get any signatures initially. That village has a particular system of management. The people don't have the right or authority to decide themselves. There is one head of the village, a hearing person who can sign very 
well, smoothly. The village head can decide matters for everyone in the village. For example, he decides about cleaning public places, electricity, water supply, all kinds of problems he is responsible for. People are not allowed to watch TV; it is edited heavily first, and there are only a few channels that are provided. This is related to their Muslim culture, where they are very strict about many things. It was this head of the village who I was working with. So I asked him, should all these people sign for their consent? But he said, no, there is no need, you can go ahead and film. Only the village head himself gave his signature; that was okay. So we negotiated and agreed that he would be the only one. Also, I film many children, and their families themselves did not understand the meaning of signatures. They didn't have a concept of research, didn't know what the Internet is. They had never seen a computer. So they couldn't understand about data being shared over the Internet, or about publishing books. So we didn't sign any forms. ... However, later they developed more awareness. So now, they can give more of their own input when they are being filmed.

This approach is more contentious because it is not clear that local customs should be able to override a general requirement for individual consent in one way or another. It is likely that we will still seek more explicit consent at an individual level later on, as deaf signers have now become more aware of our work. Until then, pictures, videos, names and other personal information are not being published.

For Mardin Sign Language, issues around video data, publications, and other forms of dissemination were discussed in the first project workshop, after a substantial amount of video data had already been collected. Participants signed consent forms translated into Turkish and, in one instance, with an additional Turkish Sign Language translation, which was filmed on video. Unlike the communities in Chican and Alipur, the Mardin Sign Language users were explicitly keen on wide dissemination of their language materials:

HD: I have discussed and agreed (with the community) on the dictionary, publications, and materials. We have not implemented this yet, but we will later on. I have been making video recordings, and I have collected signatures (for consent) from the participants. The aim is to disseminate these materials widely. The participants are very happy that the existing Mardin Sign Language will be preserved.

Of course, delaying the formal act of obtaining consent does not imply ignoring a duty of care towards research participants. On the contrary, proceeding in this way makes it all the more necessary to develop a sensitive radar as an informal way of trying to gauge the level of agreement with the research process in the community. Nevertheless, this still involves a significant element of risk on the part of the research team. For the indi- 
vidual fieldwork researcher, there is a risk that someone higher up in the "project hierarchy" may reject the use of data collected in this way, or may enforce compliance with "macroethical" procedures which are unsuitable in the field. Moreover, there is a collective risk for the research team because initially, there is no guarantee that informed consent will indeed be given as the project progresses, and despite the researchers being deaf and from the same country, they all came across cultural difficulties, conflicts, and barriers. These challenges are discussed in Section 5.

\subsection{Working with gatekeepers and mediators}

The quote from SP in the preceding section points to the importance of gatekeepers and mediators in the Alipur community, and working with such people is a well-known issue for fieldwork researchers (cf. Kusters, this volume). Being able to work with a research assistant who is a member of the target language community can be invaluable (cf. de Vos, this volume), and this is confirmed by HD's experiences, particularly at the beginning when he was not yet sufficiently fluent in Mardin Sign Language.

HD: I have grown up using Turkish Sign Language, but as far as reception is concerned, it's not at all easy for me to understand (Mardin Sign Language).

For example, there are a huge number of facial expressions. I thought this would be limited system, but it is absolutely huge. ... When I have difficulty with the research, the solution is that I need to have an assistant. I can sit with the assistant and look at things (on video) and clarify them. My assistant knows Mardin Sign Language, but she likes to get in touch with other members of her family, to show them things and ask them, like, what does this mean, what is that, to draw out the answers from them.

Of course this has not only been helpful in terms of data analysis, but also in order to engage more effectively with the other family members in any practical matters or in cases of conflict.

It is interesting that in both Alipur and Chican, communication sometimes took place through hearing interpreters. Being deaf and from the same country did not provide a sufficient basis of communication when it was important to provide exact, specific information beyond casual conversation. There is no doubt that HD, SP and CEED learned the respective minority sign language very quickly, and this direct contact through the local sign language has proved very important for establishing a rapport with the local deaf participants as well as for the quality of research outcomes. However, SP and CEED chose to use the services of interpreters for specific purposes: 
SP: I wanted to explain to them what I was aiming for, why I was doing this work, and what would be the benefit to them, trying to sign to them (with their signs), because their signs were different. So I had to adopt and learn their signs to an extent. However, there was a hearing person who came, and I used speech to the hearing person, who then signed it to all the deaf (in local signs). So the communication was through an interpreter.

This situation happened only in one particular instance, when there was a critical moment in communication during a workshop where more complex information needed to be conveyed. Otherwise, informal communication was through sign language.

CEED: I first started by questioning them, asking them what they want the most. ... So I explained this to them through an interpreter, giving the options: did they want a deaf school, or something more practical connected to material life like their local businesses and surroundings, or what did they want?

In addition, CEED chose to involve a hearing interpreter alongside deaf signers for certain linguistic tasks that required specific meta-linguistic skills:

CEED: I would show them a word and ask what the sign is, but they often responded with long sentences in their sign language. I was aiming only to get a single word and I said, no, no, tell me just in short, but they gave me long sentences. So I brought in an interpreter, because the interpreter could pinpoint and give me just the signs in short. Then eventually, the deaf understood it too, and we communicated as a group.

Gatekeepers, mediators and interpreters are perhaps more likely than other research participants to play a role during times of conflict. Section 5 deals with cultural adjustment and conflict resolution.

\section{Implementing community activities}

In this section, we discuss the various community-based activities carried out in the three communities during the project. Decision-making processes are described in Section 4.1; we discuss how these decisions have come about and who was involved. Section 4.2 goes into more detail as to the possible benefits that these minority language communities may derive from the practical aspects of this research.

\subsection{Decision-making processes}

Deciding about community projects is not easy when the researcher and the community have completely different life experiences, background knowl- 
edge, and available resources. As pointed out in previous sections, here again a lot of time is needed to arrive at a sensible and actionable decision. In Alipur, SP was involved in the re-opening of a school for the deaf. The factfinding and decision-making process involved several stages and various groups of people in the village.

SP: I noticed something about the village: in terms of education, for hearing people there was a lot of education. They were well-educated, and there were many schools all around which were really good. But what about the deaf? The hearing population is about 20,000 people, and that is a lot. There are many good schools everywhere, but for the 150 deaf, there is nothing, zero, not a single deaf school. A few people told me that previously a deaf school had opened, but after a short time, only one year, it was closed. I asked, why was it closed? They said, the teacher was teaching us in the oral way, and was speaking, so we couldn't understand anything. So people stopped coming to the school. It was also a cultural thing, as deaf people had never pursued education. They were only working as manual labourers, and there was no emphasis on schooling. So many of them skipped school, and it closed. Ok, so I thought hard about that. I knew that they all had the potential to study. I saw that there was no fingerspelling used at all, and they were completely illiterate. They could not send SMS messages on mobiles. I made a lot of such observations, and I thought, the school that had closed before, maybe it can be opened again, but how?

Here the initial phase is based on the SP's observations in the field, supplemented by additional specific information elicited from deaf people in the village. The fact that SP is already familiar with life in rural India, albeit from a different region, is particularly valuable, as this background knowledge makes it much easier to assess what level of action is likely to be successful and what local resources may be available to draw on. In addition, SP is active in the area of deaf education in urban areas of India, and has experience in establishing bilingual deaf educational programmes.

After the initial idea for the deaf school had developed, SP pursued the topic in a more systematic way, involving first a larger number of deaf people and then the village administration.

SP: I arranged a workshop where all the deaf came together. I could already sign a little in their sign language, so I started to communicate and debate with them. And they all said, yes, we can have a school and we want to have it. Both adults and children wanted the school. They understood the situation, like, all the hearing people can read and write and develop themselves well, but what about us deaf people, we have nothing, and we are stuck. Ok, so I decided about the school, but what could I do? It would be a big undertaking, needing lots of work, and funding to help set it up, so I couldn't do that. 
I thought about how to let the idea take hold. I met the leaders of the village and had a lot of negotiations with them. Finally, they agreed to provide a building, they provided carpeting, and salary payments to the teachers. In addition, there was the funding that I had obtained for the project before, and which I had paid to the village head, so it was really his. He is a very religious and honest man, so he redistributed that money as salary payments for the teachers. So that's how it developed, and now the school is running well and is well-established.

As Alipur is a relatively well-off community by local standards, it was beneficial to draw on local resources. The project resources acted as seed funding, but the continuity of a project such as this new school, which is still up and running, depends on the local administration taking over, and lobbying for this was part of SP's approach. If a project is fully resourced through external support, the risk is very high that it will disband immediately when the outside involvement comes to an end.

In the local context and for this particular purpose, this consultation process at multiple layers was feasible for the more tangible outcomes that were already familiar to the local population. Everybody was already aware of the value of education. However, for the more abstract aims of protecting a language from endangerment, a different decision-making approach was needed, as the concepts behind language endangerment and its consequences are unfamiliar in Alipur. This is evident in the decision to create bilingual multimedia materials, which included various semantic categories of Alipur Sign Language signs with translations in the local spoken language, Urdu:

SP: I had asked them whether everyone (in the village) can sign, and they said no. In a number of households, nobody could sign. Only those outside with regular contact with the deaf were able to sign, but not within all the households. In addition, the women usually stay at home, so they have much less access to signing. So I asked if everyone could access television, and they confirmed and were happy with the idea. How this would work in terms of technology they didn't know. So I made a DVD (with signs) and handed it over. They were unable to imagine what language documentation is and how the future would be for the language. I only discussed this with the village head, and we agreed about the various actions. I checked with him if everything was right, and he was happy with these things.

Again, we see how the village head acts as gatekeeper and takes decisions on behalf of the community, as is common practice in Alipur. For other field sites, without such a hierarchical structure, the situation is different. For Mardin Sign Language, a more directly participatory approach was possible, as the small group of signers can contribute more directly to decisions and their implementation. 
HD: The group of Mardin Sign Language users want to document the various (aspects of) their language. Looking at their situation, it was important that we in the (research) team first discussed amongst ourselves (...) that these people have their own Mardin Sign Language, and how to provide them with an approach to documentation, how we can film them, etc. Once we agreed this in the research team and it was clear, I approached the Mardin Sign Language family members, and they were very happy. I gave them only a very small idea, just dropping in a thought and then stepping back. I didn't bombard them with many ideas from my side. I explained language documentation to them, about filming and assembling materials.

This interaction is quite typical in that HD carefully introduced an idea and then waited for the reaction from the participants. This can speed up the decision-making process and also helps the participants to clarify options and opportunities, particularly at the beginning when they may not have enough background knowledge to be aware of the range of feasible possibilities. The decision-making process then becomes increasingly concrete:

HD: In May 2011 we had a meeting with the Mardin Sign Language group in Ankara. People came together for a workshop, and this created a tremendous set of materials. We wanted to assemble various categories of signs to put into a dictionary; that was the first thing that we (researchers) and the participants agreed. Secondly, we wanted to preserve their memories. After discussion, (we decided that) we wanted something about the local area (of Mardin), and have sign language (texts) about all the various places there. Thirdly, (stories from) our elderly participants, (about the time) when they were children, how they used to play and interact. All those memories came back to them, and we filmed a collection of their stories on video. All this we want to include in a DVD and disseminate. So we agreed these things. ...They said that they really wanted a glossary with various categories of signs, so I said yes, good idea. I accepted the ideas on what to document that they contributed, and I took a back seat. Eventually, we combined the ideas that the family members had and the ideas that our research team had and brought them together.

It is evident here that both parties, research team and language community, feed into the process. HD as the lead researcher has to keep a balance between the input from the sign language users and the background knowledge that is available within the research team, and find a compromise that works for everyone. In Section 6, HD discusses a similar complementary approach in terms of involving both deaf and hearing researchers. In this case, it has been beneficial that one member of the research team is also a member of the MarSL-using family group, so that she was able to be a mediator. 
For the village of Chican in Mexico, a dictionary of signs was one of the intended outputs, as for the communities of Alipur and Mardin, but the technological infrastructure is very different in Chican, and the researchers had to take this into account. As in the other two cases, the decision-making process was not straightforward in Chican.

CEED: I first started by questioning them, asking them what they want the most. Just to film everything and then leave ... I didn't want to do that. ... So I explained this to them through an interpreter, giving the options: did they want a deaf school, or something more practical connected to material life like their local businesses and surroundings, or what did they want? They were thinking a lot and not coming up with anything at first. I suggested they might like a book of signs, as there were none available, and they really liked this idea of the sign language dictionary. I showed them some samples, the book from Bali, and showed them the $\mathrm{CD},{ }^{8}$ so they could understand, and they wanted something similar. ... It was important to give them something, and also to have publicity for them internationally, so that people outside would understand and respect them. I asked them about making a DVD, but they said no, that wouldn't have any benefit. That is because in the village no technology is available, there is no electricity because it is a small, remote village. There is no mobile phone network coverage and no Internet. They might get it set up in the future, but as a rule, a population of 700 people is not enough (in order to get the service). If there are more people (in an area), for example 5,000, or over 2,000, then network coverage is set up. But for only 700 people, that is too small. So it was better to have material on paper as a hardcopy book. This can be kept on shelf, and can also be distributed, so it is beneficial. People can physically skim through the book and look up the signs for words, in Spanish, English, and Mayan. And there will be the Chican Sign Language signs.

To a greater or lesser extent, the approaches discussed here have elements in common with participatory research and co-inquiry in the humanities and with deaf people (see, for instance, Carleton 2007; Yorks 2007; Barnett et al. 2011). It is often pointed out that decisions about research and its applications should involve community members from the very beginning. Discussing such approaches in relation to working with the Deaf community, Harris, Holmes \& Mertens (2009) point out that "[i]nstead of having a hierarchical research team with a primary investigator at the top, assistants in the middle, and participants at the bottom, the research project should be a horizontal dialogue between research teams and participants."

However, in practice this is not easy when limited time is available for fieldwork, and our project often needed to use a variety of strategies, compromises, and sometimes short-cuts towards decision-making. The engagement 
processes with the communities took a long time. People are not able to come up with requests to researchers at the drop of a hat; they often need time and intensive engagement in order to gauge the effects and benefits that linguistic research may have for them. A "pure" theoretical notion of bottomup participatory processes does not reflect the realities of our fieldwork.

\subsection{Benefits for minority sign language communities}

So what have been the benefits for the communities of Alipur, Chican and Mardin from this research so far, or what further benefits can be expected? Throughout the project, we have been considering and re-considering this issue, which is evident in the data from the professional dialogues.

CEED: (We need) something for teaching in the school. ${ }^{10}$ The teachers who are in the school should be given some material, so they can use it themselves to teach. I can't teach myself because I have no time. So it is better to bring some material and for them to use it there locally. ... If we can print the dictionary, then afterwards they can do things themselves with the help of these materials. We can develop more ideas, and eventually set up a building, and that would be beneficial.

SP: With my work, it is similar in terms of giving something to the community. When I applied for the project, ${ }^{11}$ I added to the budget that I would make something like a dictionary. ... Actually, as I see it now, it is not a dictionary, but rather a word list. It has groups of signs, such as food, vegetables, and various word groups. I had consulted with the community before, and what they all wanted was that all the hearing people in the community must be able to sign well. At the moment, if there are deaf family members in the home, then (hearing) people living with them can sign well. But other people outside may only use some gesture and a rather low level of signing skill. So we feel it is better to disseminate the sign language widely. So okay, we collected signs, edited video clips and put them on a disk. I have made a DVD and handed it over. It is lucky that the village has its own TV channel. So we only needed a single DVD, and it can be broadcast into all homes in the village. Everyone can see it because they all have this technology. So the DVD worked out well. Secondly, the deaf school was set up. We established the school because it is very important (for the deaf) to have literacy, to be able to send SMS messages on their mobiles .... Thirdly, all the deaf, in particular the adults, are illiterate. Now the school has started to teach the children, but there is nothing for the adults. So I thought that in the evenings twice a week the adults can get together as a group and get a little bit of instruction. This was only aiming at sending SMS messages for communication, like "come", 
"go", "time", so that they could send this by mobile text. Secondly, many of them are very busy, working, having regular transactions with money, but they never write anything down or make lists. Everything only exists as signing by hand and in their memory, that's all. Information about who has borrowed money from whom, how much, etc., all this they memorise and talk about in signs only. So it is better if it can be written down, and they all want to be able to write this down. But it is difficult for them to write down everyone's names. Everybody has sign names, including all the hearing people, they have all been given sign names, but (the deaf) don't know the written names. So they face a barrier with respect to writing, and the adults need to be taught about that. ... In addition, I have been documenting the village's own sign language. I have collected many hours of videos, about 20 to 25 hours. This big collection is being kept in a big computer server in an organised way. In the future, if the language becomes endangered and is about to die and disappear - we don't know - there will be a record of the language kept safe, and they can all remember it.

CEED: Yes, that is right, I agree. If the language dies, we must be able to retrieve something from files. Later on, ... if the language has died, there will be a substantial file collection. This is important because of the background and history of the village, and there may be other similar villages around the world, so if we have substantial records, we will be able to know how many such sign languages have existed.

The Alipur and Chican sign language users may not be concerned or even aware at present that their language might become endangered, and what consequences this could have. It is the researchers who are anticipating such a situation. It is also evident that SP and CEED are using a lot of local background knowledge both in terms of the general environment and in terms of the specific situation of the deaf people in Chican and Alipur.

In addition to these various uses of language resources and considerations about literacy and education, another important impact that linguistic research can have is a change in language attitudes. This is very evident in the community of Mardin Sign Language users. As explained in Section 3, the Dilsiz family members were quite hesitant to consider the value of their language at the beginning of this research. However, this attitude gradually changed.

HD: Our participants really want to preserve their own family stories. When I asked whether this material needed to be disseminated, they said, yes, they wanted to disseminate it, so that's what we will do... We now have materials for MarSL, and there are a few people (among the family members) who want to teach the language to children. They would like to teach the sign language to hearing children. This is their own idea, and I support that. 
Of course teaching MarSL to children may or may not work out in practice, but regardless of the actual result, the change in attitude is evident from such statements. Much of this change was due to intensive interaction with groups of people, particularly during project workshops, and HD points out the importance of such in-depth involvement between researchers and language users as a group.

HD: There is a big difference now (with respect to the community), and they have benefited (from our research). In particular, I think that we should have more workshops. We have organised two workshops, but that was hardly enough. In order to give the participants more information and feedback, I would like to have further events, but we have only had a budget for two small workshops, that's all. I think there has been a huge benefit for the community. We have a very warm relationship with the family (of MarSL users). It is not enough to keep meeting the same one person over and over for research. Working with a group of people carries more benefit for the community.

Furthermore, initial work through the EuroBABEL project enabled the MarSL users to understand what is involved in such research and therefore to participate in decisions. A process which involves members of the language community as early as at the bid preparation stage is ideal, but would have been impossible at the very beginning when the MarSL users were not even aware that their "old signing" could be of relevance to anyone. HD reflects on this change in the capacity of MarSL users to engage in negotiations about the next research project bid:

HD: Initially ... I just met and engaged with the community, did some video filming, and some research and documentation, and that was it. There was a give-and-take with the participants, and they wanted to do more, so we took this on board and applied for a different project through ELDP at SOAS. ${ }^{12}$ This was a fairly big project, not a huge one, but sizeable. Before putting in this new bid, I met with one of the deaf members of the family, Hasret Dilsiz, and through her, with her parents. We discussed the bid and I was feeding them information, so we could negotiate the various aspects of the bid. We then agreed (to go ahead). We agreed what would be their job and what would be my job, and how we would combine our efforts. Subsequently, our bid was successful, and I contacted them again to start working. We have been pursuing this project over the last 14 months. I keep meeting the community a lot; I always go there frequently and meet them.

In summary, benefits from this research include user-friendly language resources, educational opportunities, and changes in language attitudes. However positive the outcomes described in this section, we have also experienced a range of problematic situations in this particularly challenging type of fieldwork situation. Some of these are exemplified in the next section. 


\section{Cultural adjustment and conflict management}

This section illustrates clearly that any naive notion of deaf people's sign language research in their home countries being an easy, unproblematic exercise is misplaced. To start with, cultural adjustment to a greater or lesser degree was necessary in all three communities. This was least marked in the case of Mardin Sign Language, as the physical environment in Turkish cities was nothing new for HD, and he was already familiar with some of the deaf Dilsiz family members, who also belong to the Turkish Sign Language using community. By contrast, SP, though familiar with rural India in general, experienced a considerable cultural shift in the Muslim village of Alipur.

SP: When I arrived there (in Alipur) for the first time and started looking around, there were many obstacles in the process. ... When I first went there and returned back home, I did some Internet research and read about the local community situation, and I became very nervous. This was because they are a very strict Muslim group, and they have many strict religious rules. For example, women are behind closed doors at home, and must wear face veils. They cannot meet and talk to any man at all. And really in the village, that's accurate; I did not see any women roaming about at all. All were at home behind closed doors. So it made me extremely nervous when I searched the Internet about this community; I was really apprehensive. ... I was always worried and nervous that maybe I might break some of the rules, like the religious rules. I was not sure if I could go to particular places or not, I didn't know the culture. In addition, I could not understand the signing of the deaf people there, I kept missing it. There were one or two or deaf people with good communication skills. For example, they were good at doing gestures and creating a mix of international signs. Still, through this communication I could not get the full information, could not get information about the culture. I was stuck, and there were a lot of obstacles for the first year and a half. After that, the doors opened, and from then until now, everything is very good. Before I was searching around on the Internet and getting nervous, but when I got there, after a while I understood that actually all the people were very nice. Really there was no bother about the religion, and no worry about the rules mentioned on the Internet, that was the wrong information. They are really all very nice people, they support the deaf, donate money, they have set up a school and given the building for it, and it is all very positive.

CEED: Were your obstacles due to the culture there, or the food, the clothes, or what?

SP: A little, but in particular, my worry was about the religion, or their rules. This is because I am a Hindu, and they are Muslims. I thought maybe I won't match with them; maybe I make a mistake and they will throw me out, or 
they will be a fight. I was nervous, how would I learn (to manage)? Then over time I learned things, because actually when I met the village Head later, he was very open. He led me around and things were open. When I asked him things, he gave me explanations. That hearing person explained to me that I didn't need to be nervous. I only needed to step back and withdraw whenever a woman passed by. I had to stand and wait and let her pass. He explained these things to me well, and I was very grateful and very relieved. Later on it was smooth.

For SP, the challenge of adjustment was purely in cultural terms and not in terms of the physical environment, as he was familiar with factors such as unavailability of hotels, frequent power cuts, and the like. CEED, who is from an urban, predominantly Spanish-speaking area of Mexico, found it a challenge to adjust both to the local culture and language, and to the way of life in a Mayan village.

CEED: In Chican, ... everything is different. They sleep in hammocks, and I did too. I learned a lot about their culture. There are a lot of mosquitoes and one issue is that it is very hot, and you need a lot of (mosquito) spray. I was always feeling hot, and always waving a hand fan for air, but it was okay. It was a strain, but I learned their culture and respected it. Once they served some food, and I didn't really want it, but they insisted that all share the same (food), so I had to respect that. I was happy with that and didn't say anything. When you sit down at the table, it is not at the usual height, it is low down and you have to squat around it. This is their culture and I learned that; it is natural there and I am happy with that.

As mentioned before, CEED would sometimes use a local interpreter to help with communication in Chican Sign Language, including when negotiating payments for the sign language consultants. For instance, it was important to find out what level of payment would be appropriate in the local context, so as to neither over- nor underpay the consultants.

CEED: We discussed what they wanted for giving me data, like food or drink. Firstly, I paid money to all of them for their work when providing data; secondly, I provided food. If there was no food, they would get tired of signing, so I provided it. So they were happy with getting paid, and felt positive themselves. I also asked the hearing interpreter what would be right, how much money should be paid. I didn't decide myself. We discussed how much should be paid, and at the end, I paid everyone the same money. I put it in individual envelopes and handed them out, and people gave signatures. People gave signatures for consent, that there was no problem with publishing their data, that their signing could be shown in public, and they said okay to that. Like, is it okay to film? Yes, okay. All were in the loop and were happy with that, and I said thank you to all. I said, don't worry, I will make a dictionary of the signs, and they will distribute the dictionary to you. This will 
be beneficial for lobbying. Because I am deaf like them, I felt a good rapport intuitively. It was good and I remunerated the deaf in a professional way.

Money also played a role in a conflict experienced by SP in Alipur. In this instance, an unfounded rumour had spread about SP's alleged misuse of video data for commercial purposes. Resolving this misunderstanding is described by SP as follows:

SP: One of the deaf adults ... said, "it is very wrong of you to take all these films, because you collect the films, and then you fly abroad and sell them". I was shell-shocked. What, me selling these sign language videos for profit?? That was the rumour that had spread, so the deaf all took that very negatively. I tried to think how I could solve this problem. I went to meet the hearing head of the village, who I had been associated with. I told him about this problem, the communication issue with people, and everything. He said, no, no, just leave it, and he called a meeting with all the deaf. He attended himself and explained through sign language that these videos that are being made for our own benefit, for the teachers teaching in the school, for dissemination, and also we can keep these signs for much later, so in 100 or 200 years you can all still see and understand them, and these are our signs. Sibaji is well versed in technology, he is a researcher. He explained all this to the deaf, and they all accepted. ...There were no repeated instances like that, only this one time.

Like CEED, SP also sought local help in dealing with payments to the consultants. As they are unfamiliar with this line of work, people may perceive that payment is too high, too low, or not on equal terms for everyone. Therefore, a local intermediary is better placed to advise a researcher from outside the community, to handle payments, and to give satisfactory answers to queries from consultants.

SP: I solved this problem by not dealing with money issues myself. I delegated this responsibility to one of the leaders in the village. I gave him the money, and he wrote down all the names and distributed all the money, managing everything himself. Then complaints stopped and it is now solved. That person can explain everything, why and how someone gets paid more, or less, and I don't have anything to do with it. Managing it in this way works much better, and there are no problems now.

\section{Deaf researchers and research teams}

The concept of "Deaf-led research" and the involvement of deaf researchers in working with communities is becoming increasingly salient (e.g. Ladd, Gulliver \& Batterbury 2003; Atkinson, Gleeson, Cromwell and O'Rourke 
2007). Therefore, it is relevant to consider how deaf researchers themselves regard their role with respect to a research setting. Previous discussion in the literature focuses on deaf researchers working in their own communities, and sometimes the approach is used in international collaboration (e.g. Leeson and Sheikh 2009). Interestingly, deaf researchers from the same country, but not from the same community within each country, are both "insiders" and "outsiders" with respect to the village sign language communities. Therefore, the experiences described by CEED, HD and SP add an interesting new angle to such discussions.

CEED discusses the building of a close relationship with a signing community as a deaf researcher, which relies on his skills in adapting to the cultural situation and developing a warm relationship with the deaf people in the village, as well as his ability to absorb the local sign language variety with relative ease.

CEED: As a deaf person, I had a good rapport with the deaf there. ... Secondly, they were very happy that I respected their signs. I consistently showed respect for their own signs, and did not try to teach them anything, and they appreciated that. ... I have more relationships with the deaf people; it is more the visual mode that's different. I also know the hearing interpreters and have a network with them, but my gut feeling is that I am linked more to the deaf people. They tell me a lot of stories, I am in their homes a lot, we talk, play games, share stories and take photos. I am also fine with the hearing people, but I spend less time with them, and it is mostly for work, it's different. This is not a problem though, and the attitude is positive on both sides.

The notion of respect appears repeatedly in our professional dialogues, and has been one of the key "ingredients" in working with these communities. Moreover, this project was also an opportunity for CEED to get training and build skills and confidence as a researcher, as is evident in some of the quotes in previous sections. The skills basis necessary to benefit from and engage with a research project develops not only in the participant community but also in the researchers.

SP comments on the various aspects of his identity in relation to the research setting in the village. Being deaf and being Indian are both considered relevant factors in the quote below, while the consequences of being a male researcher, having a different religion and not being from the village itself were discussed in Section 5.

SP: I feel that going there as a deaf researcher, and it being the first time (for research), it was a positive experience for all. They did not feel anything negative, for example that someone already came before and didn't pay me, or someone came and just took away the video data from us; there weren't any stories like that. I saw this as positive. ... Maybe later if someone else 
comes and wants to make video recordings, there could be problems; that is a different topic, I don't know. When I went for the first time, it was lucky, as I am deaf, I am Indian, and I am a researcher. So they were all happy for me to do the filming, and it was positive.

Among the several markers of identity (male, researcher, deaf, Indian, Hindu), being deaf does stand out as very important with respect to the relationship with deaf signers in Alipur.

SP: There is a community of 150 deaf people, and the hearing population is very large, about 15,000 to 20,000 people. I don't really know them, I only know a few people, those who can sign and who I have met. For example, when I walk to the school, I meet those (hearing people) who have their shops on the way to the school, selling food and drink. So when I walk past, they all see me regularly and know me. But the village is quite big, and in the more peripheral areas I don't know anyone. There may just be some casual conversation in a very limited way. With the deaf, I have got a very deep relationship. All of them know about my work, what I do, about my life, my family, we also talk about their work, and we exchange and share everything in full. With the hearing people, I have less interaction, maybe just a short question, like they may ask where I'm from, or what my work is, that's all. I do not feel a special connection. Even if they can sign, I still do not feel like communicating openly, we just talk less.

HD reports a similar immediate connection with the deaf participants in the Mardin Sign Language project.

HD: It is very easy for me to meet the deaf participants. I just look at them once, and they look back, and we have that visual contact. ... If there is a mixed team of deaf and hearing researchers, it is very easy to do a good data collection. ... The deaf (researchers and participants) will all be signing away with each other, and collect rich data easily.

The reference to "rich data" hints at another consideration, which has to do with research methodologies and the quality of data, in addition to and indeed building on community-researcher relationships. This relationship is discussed in more detail by HD, and again the notion of mutual respect is important.

HD: It is really very important to have deaf researchers. ... When I meet (a deaf participant), I feel that we have things in common, not related to the research, something personal, something that is the same about me and the other deaf person. I feel there is something different, like a difference in the way I ask questions, in the way I approach people. Yes, we do have the same culture, but also, academic work is something very different. When I interview people (for research), I feel there is quite a good attitude. ... Many deaf people regard me with a tremendous amount of respect, and they look up to 
me, and this is mutual. I contribute something, they contribute something, and there is a lot of respect and reciprocity.

However, how a deaf researcher may be regarded by the community of sign language users in the field is not always straightforward. In addition to the above quotes, HD also reports some conflicting attitudes on the part of the Mardin Sign Language community.

HD: For the Mardin Sign Language using community, seeing a deaf researcher is very puzzling. They respect it, but also can't quite believe it. They feel it is like magic that a deaf person can do research, it is something tremendous. They do not have any information about what happens in other parts of the world, that there are deaf people with MAs and PhDs who are academics. Of course all these deaf researchers are skilled, but the MarSL community does not have any information about this. I feel that they regard me in a puzzled kind of way, as if this may be a dream only. I tell them that I am a researcher, and they say, how can you be a researcher? You do not speak, you can't hear, how do you do research? I explain to them that it is a matter of cognitive capacity, and that I can function visually. I have explained that to them, but it just needs some time (to sink in). They do not yet regard it as normal that a deaf person can be a researcher, but with time, they will understand.

This kind of experience may no longer be common in the industrialised countries of Western Europe and North America, but is certainly relevant in our context. We are now familiar with lines of argumentation such as in Leeson \& Sheikh (2009:8-9), who emphasise that the specific course they are reporting on is "about Deaf people, taught by Deaf people, delivered in signed languages, and informed by Deaf-led research" and that "the key for success is partnership with Deaf academics and organisations of Deaf people". First-hand evidence about how such an approach plays out in other regions of the world is comparatively rare, and it is important to accumulate such first-hand evidence from the field in order to inform our views.

\section{Conclusions}

In this chapter, we have aimed at summarising fieldwork experiences in three communities with small-scale minority sign languages as expressed by deaf researchers working in their home countries. Rather than discussing research ethics and best fieldwork practice abstractly, the present chapter includes detailed case studies and first-hand evidence.

Many of the issues and approaches discussed here are familiar from research on spoken languages, and in particular, research on endangered languages has been concerned with issues of ethics, including within complex 
fieldwork settings (cf., for instance, Grinevald 2007; Thieberger \& Musgrave 2007). However, comparable literature on endangered sign languages is still rare. Therefore, detailed case studies from which to build a more robust data base of fieldwork practice on endangered sign languages are of particular value. As Debenport (2010:241) emphasises with respect to endangered spoken languages, "each of us involved in field-based research is incredibly closely tied to the specific variables of our own field sites, making it beneficial for us to thoroughly consider the conditions under which other research projects and community/academic partnerships are taking place". This is no less true of research on endangered sign languages.

Moreover, there is a particular need for contributions by researchers from developing countries, as many endangered languages, both signed and spoken, are located in developing countries, but the majority of linguists working on these languages have been from industrialised countries. As pointed out in Section 1, the "voice" of such participants in academic discourse is of considerable value. As and when a more extensive data base of case studies can be assembled, the field of sign language linguistics will be able to derive its own conclusions in terms of best practice and research ethics for work on endangered sign languages. Our chapter is a contribution to this emerging academic discourse.

\section{Acknowledgements}

This research has been funded by the UK Arts and Humanities Research Council (AHRC) under the EuroBABEL programme of the European Science Foundation's EUROCORES scheme (project "Endangered Sign Languages in Village Communities", project number AH/H500235/1), as well as two projects under the Endangered Languages Documentation Programme ('Signing in a 'deaf family' - documentation of Mardin Sign Language, Turkey", project number MDP0200, and "Investigation of an endangered village sign language in India: A pilot study", project number PPG0039). We are very grateful to the participants in the three communities of Chican, Alipur and Mardin for generously sharing with us their unique sign languages and cultural heritage. Hasan Dikyuva offers his particular thanks to Hasret Dilsiz, Şükrü Dilsiz and the rest of the Mardin signers for their immense contribution to the workshops, which were enjoyable, memorable and illuminating. Ernesto Escobedo Delgado wishes to thank two hearing interpreters, Audomaro Colli and Simon Colli, who translated between written Spanish and Chican Village Sign Language, and written Mayan and Spanish. He also 
expresses his gratitude to the Deaf Chican villagers for providing essential background information about Mayan cultural life. Sibaji Panda expresses his thanks to the deaf friends in Alipur who have supported his research, as well as special thanks to Fazil Raza, who as a fluent hearing signer has helped with facilitating the various stages of fieldwork and opening of the "Unity School for the deaf".

\section{Notes}

1. In this chapter, we are not using the differential spelling of "deaf" (for audiological deafness) versus "Deaf" (for cultural deafness), as we feel it does not reflect the realities of rural signing communities.

2. Obviously, "voice" is used here in a metaphorical sense, meaning the views and opinions of deaf researchers. However, we chose to keep the term "voice" because of the association with other academic literature, where this term is commonly used.

3. EuroBABEL stands for "Better Analyses Based on Endangered Languages"; see details about the project in the acknowledgements. The UK strand of the project runs from September 2009 to February 2013.

4. This was in 2005 .

5. This has important consequences for the notion of "informed consent", as detailed in Section 3.2.

6. On the video, CEED uses some Chican signs to quote how he talked to the local people, e.g. using the sign TIE+GLASSES to talk about city-dwellers.

7. With the exception of female signers in Alipur, given the cultural context (see Section 4.1 and Section 5).

8. Here CEED is referring to Marsaja (2008), a book with an accompanying CD with video clips.

9. Various labels are in use for such approaches, including Participatory Action Research (PAR), Community-Based Participatory Research (CBPR), community-participatory research, and the like (Harris, Holmes \& Mertens 2009).

10. There is a special needs school some 30 minutes' drive away that a few of the deaf children and young people from Chican have been attending, and some of the teachers there have become interested in Chican Sign Language.

11. This refers to the pilot project funded by the Endangered Languages Documentation Programme (ELDP).

12. Endangered Languages Documentation Programme at the School of Oriental and African Languages in London. 


\section{References}

Atkinson, J.R., K. Gleeson, J. Cromwell \& S. O’Rourke

2007 Exploring the perceptual characteristics of voice-hallucinations in deaf people. Cognitive Neuropsychiatry 12(4). 339-361.

Barnett, S., J. D. Klein, R. Q. Pollard, V. J. Samar, D. Schlehofer, M. J. Starr, E. Sutter, H. Yang, \& T. A. Pearson

2011 Community participatory research with deaf sign language users to identify health inequities. American Journal of Public Health 101: 2235-8.

Carleton, T.

2007 Globalization and participatory action research in the humanities: Taking 'Linguistic Field Methods' out of the classroom and into the field. The International Journal of the Humanities 5(8). 151-6.

Crasborn, $\mathrm{O}$.

2010 Ethical questions on the publication of sign language data on internet. Sign Language Studies 10(2): 276-290.

De Vos, C.

2012 Sign-Spatiality in Kata Kolok: How a village sign language inscribes its signing space. $\mathrm{PhD}$ dissertation. Nijmegen: Max Planck Institute for Psycholinguistics.

Debenport, E.

2010

The potential complexity of "universal ownership": Cultural property, textual circulation, and linguistic fieldwork. Language and Communication 30 (3). 204-210.

Grinevald, C.

2007 Linguistic fieldwork among speakers of endangered languages. In Miyaoka, O., O. Sakiyama, \& M. E. Krauss The Vanishing Languages of the Pacific Rim, 36-76. Oxford: Oxford University Press.

Harris, R., H. M. Holmes \& D. M. Mertens

2009 Research ethics in sign language communities. Sign Language Studies 9(2): 104-131.

Jones, L. \& G. Pullen

1992 Cultural differences: Deaf and hearing researchers working together. Disability, Handicap and Society 7(2): 189-196. 
Kubanyiova, M.

2008 Rethinking research ethics in contemporary applied linguistics: The tension between macro- and microethical perspectives in situated research. Modern Language Journal 92(4). 503-518.

Ladd, P., M. Gulliver \& S.C. E. Batterbury

2003 Reassessing minority language empowerment from a Deaf perspective: the other 32 languages. Deaf Worlds 19(2). 6-32.

Leeson, L. \& H. Sheikh

2009 SIGNALL: A European partnership approach to Deaf Studies via new technologies, INTED 2009, Valencia, Spain, 9-12 March.

Linguistic Society of America

2009 Ethics Statement. LSA [online]. Available at: http://www.lsadc. org/info/pdf_files/Ethics_Statement.pdf.

Marsaja, I.G.

2008 Desa Kolok: A Deaf Village and its Sign Language in Bali, Indonesia. Nijmegen: Ishara Press.

Nyst, V.

2007 A descriptive analysis of Adamorobe Sign Language (Ghana). $\mathrm{PhD}$ dissertation. Amsterdam: University of Amsterdam.

Thieberger, N. \& S. Musgrave

2007 Documentary linguistics and ethical issues. In Austin, P. (ed.) Language Documentation and Description, Volume 4. London: Hans Rausing Endangered Languages Project, pp. 26-37.

Wilkins, D.P.

1992 Linguistic research under Aboriginal control: A personal account of fieldwork in central Australia. Australian Journal of Linguistics 12. $171-200$

Yorks, L.

2007 The role conundrums of co-inquiry action research: Lessons from the field. Systemic Practice \& Action Research 20(1). 105-16.

Zeshan, U.

2007 The ethics of documenting sign languages in village communities. In Austin, P.K., Bond, O. \& D. Nathan (eds.), Proceedings of the Conference on Language Documentation and Linguistic Theory, 7-8 December, SOAS, London, 269-279. 


\section{Part II}

\section{Profiles of shared-signing communities}




\title{
Adamorobe: A demographic, sociolinguistic and sociocultural profile
}

\author{
Annelies Kusters
}

Adamorobe is a village located in Ghana, $40 \mathrm{~km}$ from its capital Accra, in a valley east of the Akwapim Ridge. Its founders are Akwamu Akan speaking Southern Akwapim Twi. Although there is an increasing number of detached houses, the village mostly consists of brick or clay houses in a traditional compound structure: rooms built around an inner courtyard, where people do everything in the open air, e.g. wash clothes, prepare food, and socialise. There is no sewage system and no water pipes, but there is electricity, a number of people have a car, and televisions and mobile phones are omnipresent.

Since 1961, several researchers and visitors have reported a high prevalence of deafness in Adamorobe (such as David, Edoo, Mustaffah \& Hinchcliffe 1971; Osei-Sekyereh 1971; Amedofu, Brobby \& Ocansey 1999; Nyst 2007). The number of deaf people counted by these visitors has varied from 34 to 45 , but has seemingly remained more or less stable. It also appears that the overall population has grown dramatically, i.e. from 405 in 1961 to 3,500 people in 2012, as a result of both births and immigration. This means the percentage of deaf people in Adamorobe has strongly declined, from $11 \%$ in 1961 to $1.1 \%$ in 2012.

The variations in deaf population - for example, I counted 41 deaf people in 2012, while Nyst (2007) counted only 35 in 2001 - is largely due to deaf people's immigration and emigration rather than from births and deaths. It appears that a number of deaf villagers reside and work outside the village on South Ghanaian cocoa farms, temporarily or permanently. Also not all deaf people living in Adamorobe were born there: at least three deaf men from surrounding villages or Accra married deaf women in Adamorobe.

In 2012, most deaf people had hearing parents (28/41) and one or more deaf siblings (26/41). It appears that this 'recessive sensorineural deafness' (Meyer, Muntau, Timmann, Horstmann \& Ruge 2001) is caused by the 'deaf gene', i.e. a Connexin $26 \mathrm{R} 143 \mathrm{~W}$ mutation which was probably spread in Adamorobe through marriages between the founding Akan matrilineal clans, starting in the late eighteenth century (Nyst 2007). 
While the total number of deaf people in Adamorobe seems relatively stable, the deaf population is ageing, i.e. it appears that fewer deaf people are born now than in the past. This is probably the result of a dual cause that impeded the circulation of the 'deaf gene': firstly, the people from Adamorobe increasingly married immigrants, and secondly, since 1975 deaf people are not allowed to marry each other anymore because deaf-deaf marriages (formerly common in Adamorobe) invariably brought forth deaf offspring. In addition, because of a local belief that deafness is passed on by men, hearing women are not willing to marry deaf men. Therefore, in 2012, amongst the deaf people aged above 20 there were more single deaf men (54\%) than women (44\%), and deaf men were less likely to have ever been married (most single deaf women had been in marriages with hearing, or sometimes deaf, partners for long periods). As a result, $87.5 \%$ of the deaf women had children with one or more partners, whilst only $23 \%$ of the deaf men had at least one child. Even though deaf-deaf marriages are not allowed by law, $26 \%$ of the deaf adults aged above 20 were in a relationship with a deaf person, i.e. four couples. Three of these four couples were childless and one couple had a deaf child.

The sign language used by Adamorobe villagers is known as Adamorobe Sign Language, or AdaSL (see Nyst 2007). Because no sociolinguistic research has taken place in this village yet, the number of AdaSL signers and their proficiency is unknown, but participant observation has highlighted some sociolinguistic patterns. Firstly, people who are able to sign especially well are close relatives of deaf people, people who grew up with deaf people, friends of deaf people, or people who work with/near deaf people (for example having adjoining farms). Secondly, there seems to be no difference between the signing proficiency of men and women. However, there are differences in age: children generally do not know AdaSL unless they have deaf (grand)parents, live with a deaf person in the compound, or have deaf neighbours. It appears that more elderly people than young or middle-aged people are proficient in AdaSL. In addition, the deaf villagers are more fluent in AdaSL than their hearing peers. Nevertheless, many hearing people are fluent in AdaSL and/or mix this language with spoken Akan. Finally, most (recent) migrants do not know AdaSL.

In everyday life, deaf people interact naturally with hearing people through sign language, but the majority of them also frequently engage in deaf-only conversations. These conversations generally last considerably longer than deaf-hearing interactions. Most deaf-hearing interactions are rather short; longer ones typically consist of one-to-one conversations in which the hearing interlocutor is particularly trusted by the deaf person. Hearing people do not usually participate in deaf group conversations. 
Deaf villagers express that "deaf people are the same", sharing their first language and certain ontological experiences, and feeling connected and united. However, at the same time, something that is very strongly emphasised by both deaf and hearing people is deaf-hearing connectedness and unity in Adamorobe. They stress that the villagers are as one, a family, and that they do all kinds of things together, communicating through sign language.

Adamorobe is thus a deaf-inclusive place. At the same time, these local perspectives and practices are supplemented by (or sometimes contrary to) global processes, agents and discourses (see Figure 1).

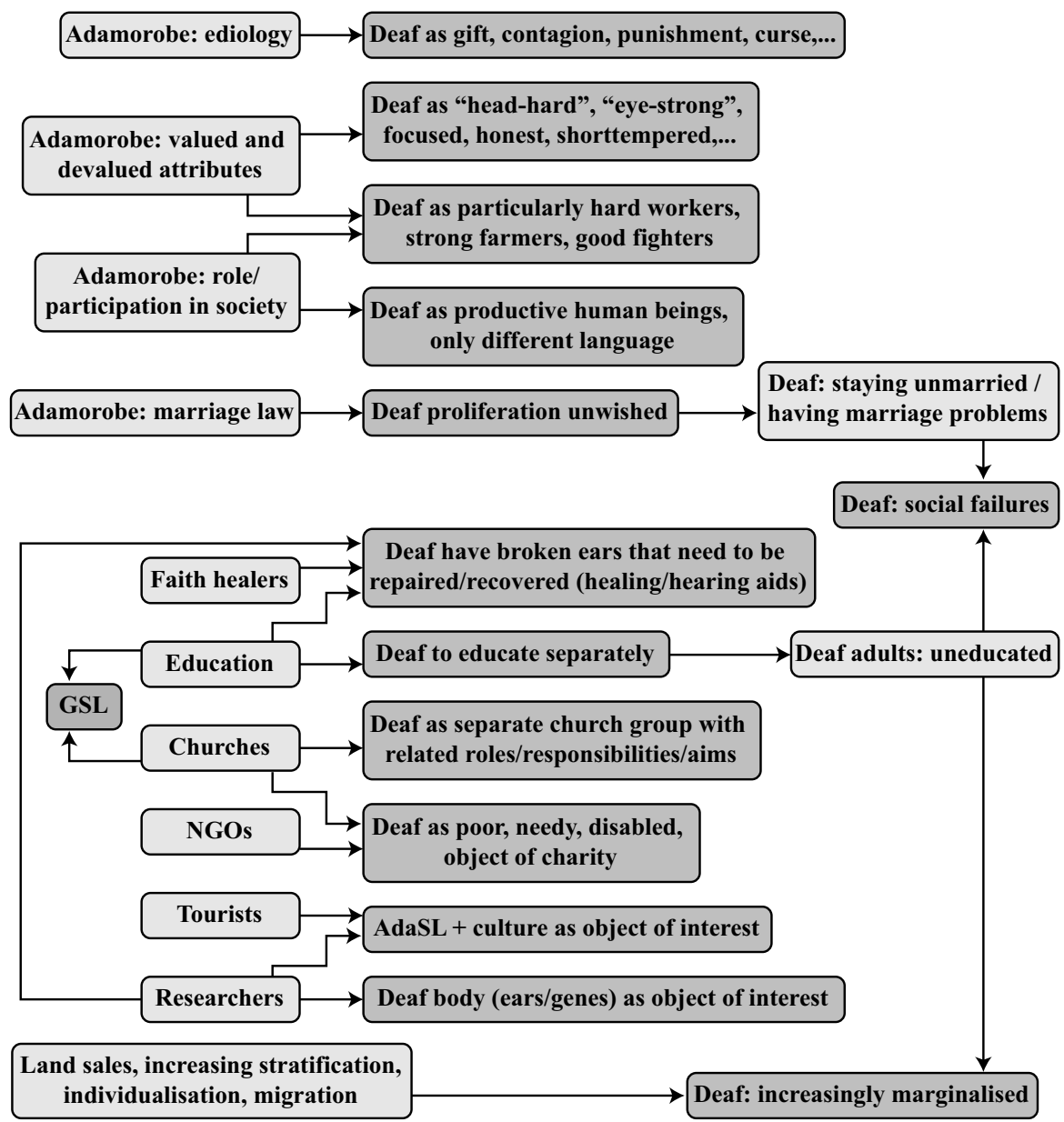

Figure 1. Perspectives on deafness in Adamorobe 
Processes that have caused the marginalisation of deaf people in the West, and constructed them as disabled, have influenced deaf-deaf and deaf-hearing social practices in Adamorobe. Capitalism, Christianisation and commodification of lands are associated with increasing fragmentation, stratification and individualism in Adamorobe, as well as a diversification of employment options and greater importance being placed on education.

In religious and educational contexts, deaf people are now separated from hearing people, which was not the case in the past, with traditional Akan religion and family-based education. In 2009 there were about 15 Christian church groups active in Adamorobe, one of them specifically for deaf people, led by a Lutheran pastor who comes from Accra weekly. As regards education, the three schools in Adamorobe are not accessible to deaf villagers. Between 1963 and 1980 there were a few failed attempts at establishing education for them (both inside and outside the village). Since 2000, deaf children from Adamorobe routinely attend the boarding school for the deaf in Mampong.

Adamorobe was introduced to the concepts of deaf church groups and deaf education by the American educator Andrew J. Foster, who arrived in Ghana in 1957. As a result, many deaf adults know some Ghanaian Sign Language (GSL), which - at least in the initial stages of its development - has been strongly influenced by ASL). The deaf schoolchildren use GSL as their first language, which renders AdaSL potentially endangered (Nyst 2007). Nevertheless, in Adamorobe, GSL does not seem to have a higher status than AdaSL. Both the deaf adults and schoolchildren believe it is beneficial and prestigious to be bilingual in both sign languages.

Churches, NGOs and visitors often aim charitable donations and development projects at the deaf villagers, but this tends to construct them as needy, and aggravates the difference between them and their hearing counterparts, whilst it appears that in the past, deaf and hearing villagers were farmers with similar incomes. Nowadays, deaf adults in Adamorobe feel disadvantaged because they are nonliterate and their array of possible life choices is narrower than that of educated hearing people. Many hearing people in Adamorobe have small businesses; work as tailors and seamstresses, hairdressers, carpenters, and teachers; and/or commute to and from Accra daily. But most deaf people are subsistence farmers, which seems to be insufficient for esteem in Adamorobe anymore. Due to the rapid expansion of Accra upto Adamorobe's vicinity, especially in the last few years, almost all the lands on the hills surrounding the valley where Adamorobe is located, are now sold, mostly to estate developers. Deaf (and hearing) people who had their farms on these lands, now need to go far from the village for farming, 
which further marginalises them and the profession of farming. Deaf people also have ceased to have roles as town guards or warriors. In addition, due to the high number of migrants that come with Accra's expansion, and the general stratification and individualisation, relatively fewer hearing villagers know sign language well, and deaf-hearing interactions are less frequent and shorter in duration. The aforementioned marriage law also marginalises deaf villagers.

However, in everyday life, the people of Adamorobe still lead their lives side by side. Differences between deaf and hearing people, ambiguity in social interactions and historical changes in deaf-hearing relationships are prevalent, but in the end, these people choose to celebrate unity and 'sameness'.

\section{References}

Amedofu, G. K., G W. Brobby \& G. Ocansey

1999 'Congenital Non-Syndromal Deafness at Adamarobe, an Isolated Ghanaian Village: Prevalence, Incidence and Audiometric Characteristics of Deafness in the Village (Part I)', Journal of the Ghana Science Association 1(2). 63-69.

David, J. B., B. B. Edoo, J. F. O. Mustaffah \& R. Hinchcliffe

1971 'Adamarobe - A 'Deaf' Village', Sound 5. 70-72.

Meyer, C.G., B. Muntau, C. Timmann, R. D. Horstmann \& G. Ruge

2001 'Skin Changes Associated with GJB2-R143W Mutations in Ghana: Evidence for Balancing Selection of Hearing Impairment' in Bernard Nocht Institute for Tropical Medicine: Scientific Report 2000-2001, 78 .

Nyst, V.

2007 A Descriptive Analysis of Adamorobe Sign Language (Ghana). Dissertation (PhD). University of Amsterdam.

Osei-Sekyereh, P.

1971 Adamorobe Research Project. Paper presented at the Scientific and Educational Conference by Ghana Association of Audiology, Rehabilitation and Education of the Deaf, Mampong-Akwapim, Ghana, June 3-5. 


\title{
Alipur Sign Language: A sociolinguistic and cultural profile
}

\author{
Sibaji Panda
}

Alipur Sign Language (APSL) is an indigenous village sign language used in Alipur, in the state of Karnataka in southern India. Alipur is a large village of approximately 20,000 inhabitants, of which 150 are deaf. The village is located 80 kilometres north of the garden city of Bengaluru (Bangalore). The village is well known in the area for its gemstone cutting and polishing. Its main spoken languages are Urdu and Hindi, and villagers have varying degrees of familiarity with the language of the surrounding area, Kannada (a language from the Dravidian family).

Alipur is a small Shia Muslim enclave in a predominantly Hindu area, which is linguistically and socially isolated from the surrounding area. This makes the village rather unusual, and there are no other known cases of religious minority communities with a high incidence of hereditary deafness in India. All of the villagers belong to the Shia Muslim community, except for a few families who are from other religions including Hinduism.

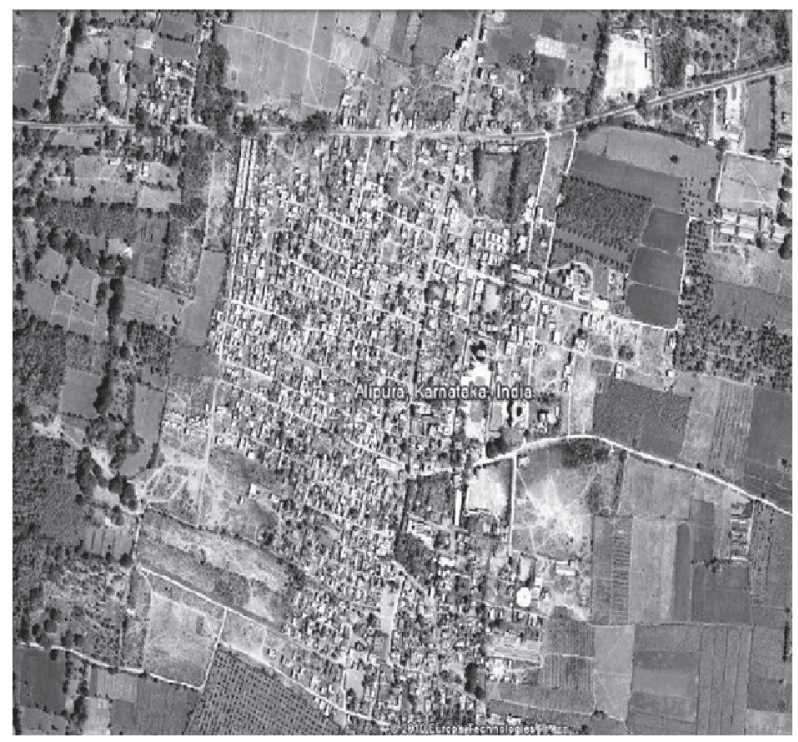

Fig 1. Map of Alipur as seen on Google Map. 
Alipur is believed to be 300 years old, and was established when Islamic sultans ruled the area. In the pre-independence period, Shia Muslim communities in India were a minority within the Muslim community itself. After establishment of the Madrasa ${ }^{1}$ in Alipur, many people of Shia faith moved to Alipur and settled down for reasons of safety, cultural and religious integrity, and economic occupations. After independence, the population and economy of the village grew rapidly, with trade attracting many migrants. The main occupations are farming as well as carving, polishing and selling precious stones. The precious stone trade has traditionally been an important type of occupation in Alipur, and a few individuals own large businesses including overseas. However, there are hundreds of small traders, and small stone cutting and polishing industries exists in the residential homes. Many of the deaf people in the village have taken up stone carving and polishing as their vocation and get paid well for the work they do, as they are often considered to be good skilled workers in the industry. Some of the deaf people in Alipur have a considerable range of vocational skills. For example, one of the deaf men in the village is skilled in construction work, making tiles, electricity repairs and fitting, carpentry, and plumbing. Getting work has not been difficult for the deaf, as they say people know their skills and contact them easily, particularly through the village tea stall that is run by a deaf man.

Whereas most Indian villages still have huts and hamlets, the houses of Alipur have concrete roofs, and there are a few high-rise buildings and mansions owned by the millionaires of the gemstone industry. Two huge Madrasas (an Anjuman-e-Jaafria and Madrassa Hussainia) in the village are testimony to the religious faith of the Alipuri community. The village has more than a hundred shops and businesses, and recently acquired a hospital, post office, and bank. Alipur has several schools, and both males and females have access to education. A few schools offer instruction in English at secondary level, which is comparable to the education available in urban areas. One of the schools is an English medium school and is affiliated to the Indian Central Board of Secondary Examination, which means the school offers quality education to the villagers. The schools, hospitals and other institutions for public services are run by a number of village trusts established by the villagers and based on their faith of serving their community. In rural India, it is quite rare for a single village to possess a range of such public service facilities.

Alipur has long had a high incidence of deafness, due to generations of endogamous marriage patterns stemming from the village's religious customs, isolation, and beliefs about outsiders. Currently, there are 150 deaf villagers, who comprise nearly $1 \%$ of the total population; this is $10-20$ times 
higher than the usual proportion seen in the general population. Deafness occurs throughout the village, but is more strongly represented in particular families, some of which have had deaf members for several continuous generations. One of these is referred to as 'the deaf clan'.

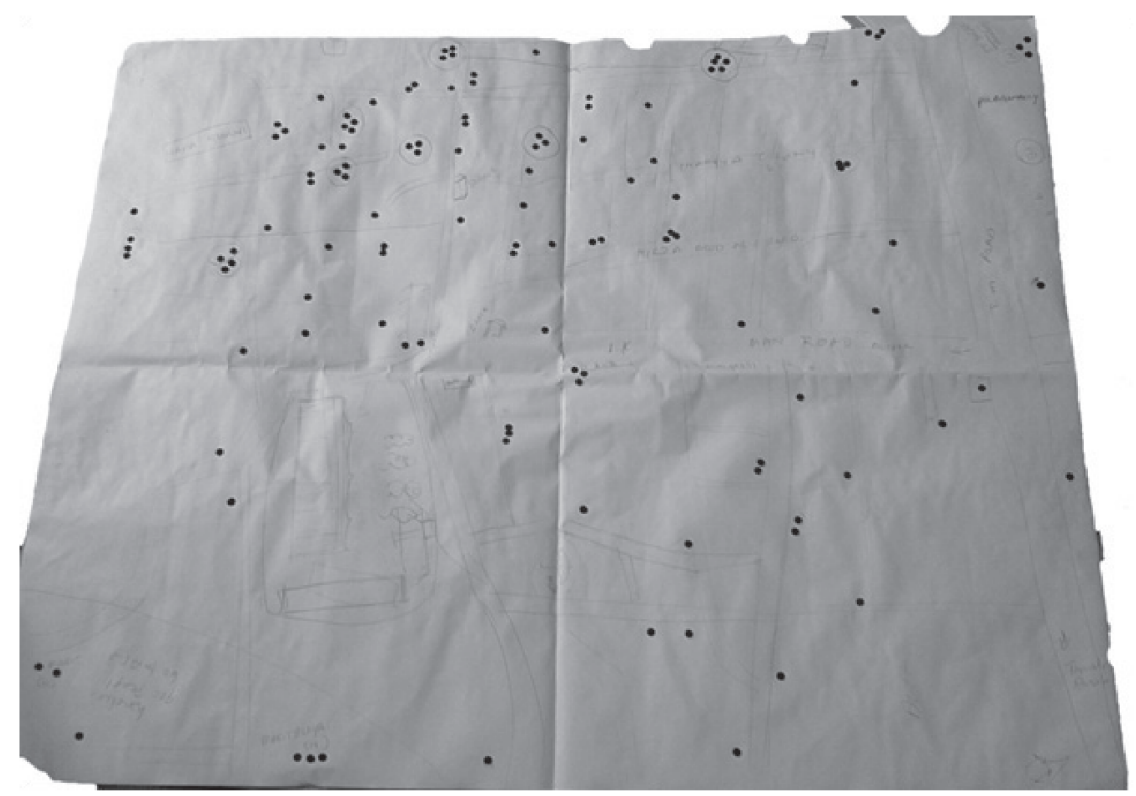

Fig 2. Map of Alipur showing location of Deaf inhabitants. ${ }^{2}$

APSL is quite different in structure from Indian Sign Language, which is used in the areas around Alipur. Due to the longstanding high proportion of deaf inhabitants, the majority of villagers use APSL (albeit with varying degrees of fluency), regardless of their hearing status. This distinguishes Alipur from other Indian villages where deaf individuals use various ad-hoc manual communication systems known as Home Sign (Jepson 1991). While APSL's sociolinguistic situation is somewhat unique, similar cases have been noted, such as that of Chican Sign Language in Mexico, Kata Kolok in Bali, Adamorobe Sign Language in Ghana, and Mardin Sign Language in Turkey (cf. chapters in Part I of this volume). Deaf APSL users tend to be monolingual, with minimal (if any) literacy, and until very recently, no access to formal education. ${ }^{3}$

One of the unique characteristics of the village is the interaction and communication between the hearing and deaf villagers. Though no specific survey has been conducted, it is obvious that the sign language community is considerably larger than the group of 150 deaf inhabitants in the village. 


\section{Sibaji Panda}

Sign language is used in all spheres of life, where both deaf and hearing people often communicate without much difficulty. A few of the hearing villagers are particularly fluent signers, but most people seem to communicate in APSL well enough to fulfil basic communicative needs. Most of the fluent hearing signers are immediate relatives of deaf people, such as hearing people with deaf siblings. Most retailers in Alipur seem to have some level of signing proficiency in order to communicate with deaf customers. Due to the extensive network of multiple cross-cutting relationships between extended families, all families have some connection or other with deaf relatives, either immediate or further removed (e.g. a second cousin).

This situation has resulted in a need for communication with deaf individuals for almost all villagers, who are exposed to sign language in various forms and with greater or lesser limitations. Recently, there have been efforts to promote the use of APSL in Alipur more actively. Interestingly, Alipur has its own television channel (Ali Channel), which recently began broadcasting some APSL signs as a community initiative (see Dikyuva, Escobedo Delgado, Panda \& Zeshan, this volume). The multimedia video with APSL vocabularies and dialogues has been helpful for spreading APSL among the villagers.

Villagers contend that APSL is as old as the village itself. The majority of the deaf and hearing villagers believe that deafness in Alipur began when an early settler arrived with a deaf relative from Iran, to find a wife for the latter. The oldest deaf man in the village, who is in his 70s, says his grandparents were sign language users. This suggests that APSL has existed for at least six generations. Further investigation is needed to shed light on exactly when deafness occurred in the village for the first time. Within the 'deaf clan', the family tree that was constructed during fieldwork shows deafness going back at least six generation.

Comparing the setting of various rural sign languages with each other, Nonaka 2009:212 observes that "[t]he communities where indigenous village sign languages have appeared are geographically, culturally, and linguistically diverse, yet they share a remarkably similar constellation of socioeconomic and demographic features". However, in Alipur this setting appears to be changing, as recently there has been increasing language contact with urban sign language varieties, particularly those used in nearby city Bengaluru (Bangalore). Two deaf villagers have studied there, one for six years and one for six months. Many others work outside Alipur (especially in Bangalore), where they also tend to socialise with the local deaf community. Due to the internet, films of other sign languages are now available to the deaf villagers, and teachers at the deaf school often use American Sign Language (ASL) 
instead of APSL. Contact has also occurred because of deaf people from other parts of India visiting the village, including a deaf researcher. APSL's number signs may have already been affected by this (Zeshan et al, in prep.).

Observations from initial fieldwork suggest that in Alipur, deafness is more of a social disadvantage than in comparable villages like Chican (cf. Escobedo Delgado, this volume). The local arrangements for dowry payments, for instance, differentiate between deaf and hearing villagers (of both genders). Marriage customs in Alipur appear to discriminate against deaf villagers, because they normally pay a higher dowry (typically cash and jewellery) to 'compensate' for their deafness. Hearing women pay less than deaf women, and hearing men pay nothing at all if they marry a deaf girl, while deaf men must pay. In addition, marriage between two deaf people is discouraged, because the villagers believe this lessens the further spread of deafness, and they doubt whether a deaf couple would be able to cope with the practical aspects of parenting, e.g. day-to-day communication. Deaf villagers express unhappiness with this arrangement, and say that being free to marry deaf people would enable them to escape the discrimination of the dowry system. However, they also fear that life would be difficult and may lead to having deaf children if they are married to a deaf spouse. Deaf men and women in the village are married to hearing partners and lead a successful family life. There are usually no communication barriers as most hearing people tend to know sign language or are ready to cope with the communication demands after marriage. These marriage patterns have been part of the social customs in the village for centuries, and are not seen as a social stigma.

Traditionally, deaf people were not thought to be educable, and deaf boys start working at a very young age. Nevertheless, in the past few years, education has become available to the deaf children of Alipur. Around 2001, a deaf school was established in the village, but it closed after only one year as the teacher could not use APSL. Since 2008, a deaf school has been open in which boys and girls are educated separately and taught by deaf teachers. Deaf children are now acquiring English literacy, with APSL as the medium of instruction. Progress of the students who attended has been remarkable considering the limited amount of time they spent in the school, the limited resources and shortage of APSL-using qualified teachers. Within the national education scenario, deaf children in India do not receive education in their own language i.e. Indian Sign Language, and do not attain the expected educational standard. However, with very limited efforts and resources, APSL users taught by deaf teachers have been able to attain a functional level of literacy relatively quickly. 


\section{Sibaji Panda}

Deaf people participate in local trade and business, but appear disadvantaged in terms of employment, perhaps due to their lack of formal education and insufficient numeracy and literacy in the local language. Most deaf people work as labourers in the gemstone or construction industries, although some of the deaf people work as tailors or shop-keepers, including two who run their own small groceries. There are several focal points in the village where deaf men meet regularly; for example, one deaf man runs a tea stall where male villagers sign with each other, particularly in the evenings.

Gender separation is strong in Alipur, and deaf women have fewer opportunities to socialise and sign outside the immediate family. Therefore, it is possible that there may be differences in the signing of deaf men and deaf women in the village. However, opportunities for conducting research on APSL with women in the village are quite limited, in particular for male researchers, so no data or information is available so far on whether there are any relevant gender distinctions in APSL.

Research on APSL and the Alipur community is very recent (see Panda 2010; Dikyuva, Escobedo Delgado, Panda \& Zeshan, in prep.). The first visit by a researcher took place in 2007, and subsequently, a pilot project funded by the Endangered Languages Documentation Programme began in 2008, marking the beginning of research on the language and the community (Panda 2010). At some stage, geneticists visited the village and collected DNA samples, but no publications are known to have resulted from this. Expeditious study is crucial because of the aforementioned increasing contact with urban sign language varieties, which is an indicator of potential language endangerment.

Recently, sign language varieties used by the younger generation and the older generation are diverging rapidly. A number of factors are responsible for this development. Firstly, younger deaf people from Alipur have become increasingly mobile and frequently travel to the city (Bengaluru), where they meet deaf friends who use American Sign Language (ASL). ${ }^{4}$ Deaf people can now travel more easily because of the complimentary bus pass given to disabled people by the state government, which means that deaf people do not have to pay for any travel within the state. Secondly, the two deaf teachers at the deaf school in Alipur often use ASL in the school. One of them is a native of Alipur who was educated in Bengaluru up to high school level and uses both APSL and ASL. The other teacher is an ASL user from Bengaluru and has acquired APSL after coming to Alipur. Therefore, ASL influence has affected the deaf children in Alipur who attend the deaf school. Finally, internet has become available on mobile phones, and the deaf signers are fascinated with the other sign languages that they can see on videos posted on the internet. 
The above factors pose a serious threat to APSL and might affect the language's vitality in the near future. When the first sign language data were collected in 2007, there was no literacy among deaf people except for two individuals who attended a deaf school outside Alipur, and no influence of other sign language was noticeable. For instance, no fingerspelling was observed in the first data collected in 2007. Some deaf people of the older generation have started complaining why the children are "using strange Bengaluru signs". Now communication is sometimes difficult between younger deaf people with ASL and English skills and those who are sole users of APSL and have no knowledge of ASL and English.

In recent years, literacy education has resulted in improved access to information, independence, and opportunities, and this is appreciated by most deaf people in Alipur, as well as the parents of deaf children. Students who attend the deaf school can now read and write, exchange text messages, and are members of social networks on 'facebook'. On the other hand, it is possible that with increased language contact, APSL will soon become endangered. The Alipur community is now aware of this potential development through discussions in workshops, and the future of APSL will depend on the collective linguistic decisions of the community.

\section{Acknowledgements}

My sincerest thanks go to the people of Alipur for their kindness, patience, support, cooperation in all stages of the research, and making me feel at home during my field visits. My special thanks go to all the deaf people of Alipur, especially those who have been with me continuously during my research work and have provided data and input. My special thanks also belong to Mr. Fazil Raza, who as my consultant has been helpful and cooperative during the fieldwork and research. I would also like to extend my gratitude to Prof. Ulrike Zeshan, who has been guiding me throughout my research work. The field research was supported by a grant from Endangered Language Documentation Project (ELDP PPG 039) and a grant from the UK's Arts and Humanities Research Council under a Collaborative Research Project within the European Science Foundation's EuroBABEL strand of the EUROCORES programme (grant number AH/H500235/1). I also would like to express my sincere appreciation to my colleagues in the EuroBABEL project for their continuous support and encouragement. 


\section{Notes}

1. A Madrasa is a school for Islamic education, and can also be used as a place of worship.

2. This map was created in Alipur by several consultants from memory and therefore does not represent all deaf inhabitants exhaustively.

3. The Unity School for the Deaf was opened in 2008 to impart literacy education in English. However, education has not been formal and the deaf school has not been recognised officially as a special needs school yet.

4. ASL is used widely in the nearby city of Bengaluru by those deaf people who have been through formal education. ASL has been used in schools and religious services since the post-independence period. This is unusual in India, where Indian Sign Language is used in virtually all urban areas. However, Bengaluru is one of very few places where there is a limited spread of ASL due to language choice in particular deaf schools. All deaf signers in Bengaluru who use ASL are also fluent in Indian Sign Language, and outside the city, only Indian Sign Language is used.

\section{References}

Dikyuva, H., C. E. Escobedo Delgado, S. Panda \& U. Zeshan

in prep. Cardinal numerals in village sign languages - Approaching crossmodal typology.

Jepson, J.

1991 "Urban and Rural Sign Language in India." Language in Society, $1 / 2.37-57$

Nonaka, A.M.

2009 Estimating size, scope, and membership of the speech/ sign communities of undocumented indigenous/village sign languages: The Ban Khor case study. Language \& Communication. 29. 210-229.

Panda, S., ed.

2010 Sign languages in village communities. Nijmegen: Ishara Press. 


\title{
Algerian Jewish Sign Language: A sociolinguistic sketch
}

\author{
Sara Lanesman and Irit Meir
}

Algerian Jewish Sign Language (AJSL) is a sign language that developed in a Jewish community in the Algerian city of Ghardaia. As the entire Jewish community in Algeria left the country by 1962, the language migrated with its users, and is in use today mainly in Israel and in France. AJSL, then, is a "village sign language" without a village. The sociolinguistic sketch of the language refers both to its development in its original locale, Ghardaia, and to its maintenance in present-day Israel.

\section{AJSL in Algeria}

Ghardaia is located in the northern Sahara Desert region of Algeria, in the M'zab area. It was founded in the $11^{\text {th }}$ century by Berbers belonging to the Ibadiyya sect, a schismatic Muslim sect who is characterised by a puritanic interpretation of the Koran (Briggs \& Guède 1964:9, Nagel 2004:27). According to M'zabite and Jewish oral traditions, Jews arrived at Ghardaia, the main town in the M'zab area, during the $14^{\text {th }}$ and $15^{\text {th }}$ centuries, and established a Jewish community there. The community lived in its own walled quarter (called mella) in the south-east part of the town. Though members of the Jewish community maintained commercial and economical relations with their Muslim neighbours, marriage was strictly within the community, which gave rise to a closed and rather isolated community. This social isolation that lasted for at least 500 years gave rise to a community with several distinct physical characteristics, among them elongated heads, slight tendency towards blond or red hair, and deafness. In 1954, the Jewish community numbered 1,091 members, and the entire population of Ghardaia was about 12,500 (Briggs \& Guède 1964:9). At that time there were 25 deaf individuals in the community $(2.5 \%)$. We do not have information about the distribution of deafness within the families in the community, but all the people we interviewed have deaf relatives, and therefore it can be deduced that deafness ran in specific families. However, according to Briggs and Guède's (1964:12) description, deaf people participated in social events and "had about as rich a 
social life as anyone". From interviews we conducted with nine AJSL signers (see chapter XX in this volume) we learn that hearing people could sign very well, and the sign language that arose in the community served as the major means of communication between deaf and hearing members.

Based on the interviews we conducted, we further learn that deafness goes at least 5 generations back in the community. However, it is difficult to get more precise information about the interaction of the deaf people in Ghardaia with other deaf people, since all the people we interviewed had left Algeria about 50 years ago, and could not recall whether there was any contact with deaf people from outside the community. Furthermore, there are no sources of information about Algerian Sign Language, ${ }^{1}$ and we have no information about deafness in the Muslim population of Ghardaia. Therefore it is difficult to establish whether the language developed de novo or was influenced by other signing systems. The Jewish community in Ghardaia had social contacts with at least two other Jewish communities in the area, the communities in Laghouat and Aflou. Deaf people in these communities also use AJSL.

Deaf children, boys and girls, in Ghardaia did not go to school, and therefore remained monolingual in AJSL. The hearing members of the community used their dialect of Arabic for everyday communication. The hearing boys, who went to school, studied Hebrew as well, in order to read the scriptures, and some of them also eventually acquired French for business discussions. Most of the hearing girls stayed at home, and therefore were not exposed to languages other than those used at home (spoken Arabic dialect, and AJSL if there were deaf people in the close surroundings).

The financial status of deaf people did not differ significantly from that of hearing people. Deaf men held ordinary jobs such as goldsmiths and porters in the market. Some were quite wealthy, while others were poor. Deaf people, men and women, were married to hearing spouses. Therefore, it seems that deaf people were integrated into the hearing community, AJSL serving as a main means of communication, used by both deaf and hearing. Nonetheless, deafness was considered as a punishment from God. According to the local superstition, deafness may be the result of having sexual intercourse during menstruation, which is considered a serious religious offence.

\section{AJSL in Israel}

Between the years 1943 and 1962 the entire Jewish community left Ghardaia and immigrated, mainly to Israel and to France. The first wave of immi- 
gration was between 1943-1950. Due to growing tension between Berbers, Muslims and Jews in the M'zab area and in Algeria in general, 500-600 Jews immigrated to Israel and France (Briggs \& Guède, 1964). In 1950-1951 the tensions in Algeria diminished to some extent and the Jews stopped leaving Algeria. Some immigrants who were unsatisfied with life in Israel returned to the M'zab region at that time (from the archives of Beit HaTfutsot \#73772).

In the 1950 s, a second wave immigration began, motivated both by the establishment of the State of Israel in 1948, and by the Algerian War of Independence with France. The Jews of the region were regarded as French allies, and as such they felt increasingly unsafe in their homes and began to leave, again. The last wave of immigration from Algeria to Israel was in 1962. No Jews remain in Algeria today.

There are no sources concerning deaf Algerian immigrants in Israel or in France other than the interviews we conducted. Therefore all the information about the lives of deaf Algerian immigrants in Israel is based on these interviews.

The emigration from Algeria changed the life of the community members in every aspect of life. First, the community itself disintegrated. Part of the community immigrated to France while the other part moved to Israel. Those who moved to Israel settled in different places in the country. Thus, members of the Ghardaia community no longer shared a physical location, and consequently the close-knit relationships between the community members collapsed. Secondly, they had to learn a new language, Hebrew, to adjust to the fact that the Jewish society in Israel was, by and large, secular, and they had to find housing and jobs.

The deaf members encountered other deaf people, who used a different sign language, Israeli Sign Language (ISL) and were part of a Deaf community with Deaf clubs and activities held by and for members of this community. Many of the members of the Deaf community were educated, an issue that was thorny and painful for the Algerian immigrants. AJSL users felt that they were singled out and stigmatised because of their origin and language, and consequently stopped using the language with other ISL signers.

Today almost all AJSL users are bilingual in ISL and AJSL. We do not have demographic data concerning the number of current AJSL signers in Israel, and their marriage patterns. However, from the interviews we conducted, we learnt that these signers use AJSL mainly with their nuclear family, both with hearing and deaf family members. They use ISL for interaction with members of the Israeli Deaf community, and since many deaf Algerians married deaf non-Algerians, they use ISL with their spouses and children too. Even in families where both spouses are of Algerian origin, the 
deaf children use ISL, and barely understand AJSL. According to our investigation, there are hardly any people younger than 50 that can use the language fluently. AJSL is not used in schools, nor is it used in any official meetings of the Deaf community. No official resources (such as interpreting services) are available, and many people, including members of the Deaf community, are unaware of its existence. The first public mention of the language was in a conference on multi-culturalism and multi-lingualism in deaf communities in Israel, held by the University of Haifa (April 14, 2008). Currently there is a growing interest in the language among AJSL users, and some of them are eager to participate in projects aiming to document the language.

\section{Notes}

1. Ethnologue (http://www.ethnologue.com, accessed in 2012) has just one line on Algerian Sign Language: "It has influenced the deaf community in Oujda in northern Morocco". Other internet sources inform that Algerian Sign Language was recognised in 2002 as the main means for communication for the hearing impaired community (http://www.conseilconstitutionnel-dz.org/languagesof-algeria.htm , accessed in 2012), and that it developed from French Sign Language, as is evidenced by lexical similarity (over $50 \%$ of the signs). Yet they do not cite any sources for this statement, and they do not provide any description of the language (http://www.sourds.net/2010/09/28/\%C2\%ABlalangue-des-signes-algerienne-est-une-revendication-des-sourds $\% \mathrm{C} 2 \% \mathrm{BB} /$, accessed in 2012).

\section{References}

Briggs, L. C. \& N. L. Guède

1964 No more forever: A Saharan Jewish town. Cambridge, Mass.: The Peabody Museum.

Nagel, R. L

2004 Jews of the Sahara. Einstein Journal of Biological Medicine 21: $25-32$. 


\section{Al-Sayyid: A sociolinguistic sketch}

\section{Shifra Kisch}

The village of Al-Sayyid is located in the northern Negev, the southern arid region of present-day Israel. Its Bedouin inhabitants are all related through kinship and named after their common ancestor who settled here in the mid$19^{\text {th }}$ century. Al-Sayyid is home to a shared signing community where deaf and hearing signers use Al-Sayyid Bedouin Sign Language (ABSL), an indigenous sign language that has emerged over the past 90 years.

Following the practice of cousin marriages, Al-Sayyid's grandchildren intermarried: four of these unions bore deaf offspring. The first deaf siblings were born between 1924 and 1940. The Al-Sayyid are now estimated ${ }^{1}$ to number 4,500 men, women and children, of whom nearly 130 are deaf. These deaf individuals are distributed throughout Al-Sayyid's major lineages representing five apical ancestors, each a son of Al-Sayyid the founder.

To this day kin-endogamy (between and within these lineages) accounts for over $60 \%$ of marriages (Kisch, this volume). Genetic research, ${ }^{2}$ conducted among the Al-Sayyid in the early 1990s, identified autosomal, recessive nonsyndromic deafness, associated with intermarriage. Demographic and genealogical data I have been recording since 1995 show a stable incidence of congenital deafness of $2.5-3 \% .^{3}$ The recessiveness of the genetic mutation, the fact that the majority $(80 \%)^{4}$ of deaf individuals were born to hearing parents, and the fact that deaf adults were until recently always married to hearing partners, further contribute to the blending of deaf and hearing.

Not only did kin-endogamy induce high incidences of deafness, it also accounts for a dense social network in which both hearing and deaf people are embedded. Many members of the community have at least one deaf individual among their household, in-laws, peers, or neighbours. Consequently, all deaf and many hearing Al-Sayyid infants are exposed to signing from birth, within the family environment, with additional (deaf or hearing) adult models in the community.

Sited on one of the highways connecting the northern Negev and the Dead Sea, the village of Al-Sayyid lies less than 20 kilometers from the Negev's district capital of Beersheba. In fact, this highway divides the original village into two parts and beside the road are situated several garages, grocery stores, and one of the village's four mosques. In the village there are several smaller 
grocery stores. The larger part of the village, south-west of the highway, ${ }^{5}$ is spread at the foot of a moderate hill, on top of which the village's first school and clinic are located. The village now has three elementary schools and two (HMO) clinics. Until 2005, Al-Sayyid was one of the many Bedouin villages officially unrecognised by Israeli authorities due to the state's refusal to recognise or negotiate land claims. Despite the current process towards recognition, ${ }^{6}$ most parts of the village still lack basic infrastructure, including paved roads, running water, connection to the national electricity network, sewage and waste disposal.

Most homes are modest cement-brick constructions of two to four rooms with flat tin or concrete roofs. There are also several new two story red tiled houses belonging to the few more affluent residents. ${ }^{7}$ Generally, village homes are arranged in compounds of several households of extended families. The village is made up of several dense clusters of multiple compounds, as well as slightly more dispersed compounds. The geographical distribution of the residential clusters partially corresponds to the five main lineages. Many dirt roads crisscross the village leading to its residential clusters, neighboring villages and the adjacent highway. Most households have a television ${ }^{8}$ and, increasingly, more men and women have mobile phones, but only a few have an internet connection. In most (multiple-household) compounds there is at least one car owner.

Formerly semi-nomads, the Negev Bedouin are the native Arab inhabitants of the Negev. The Negev Bedouin have a very high proportion of young people; more than half of the population is under the age of $14 .{ }^{9}$ Fertility rates are high and kin-endogamy and polygyny are common. The Negev Bedouin constitute one of Israel's most marginalised minority groups. Bedouin (men) are mostly enrolled in the lower strata of the labour market. With a population of over 210,000, the Negev Bedouin make up roughly a fourth of the otherwise predominantly Jewish-Israeli Negev inhabitants.

Most deaf and hearing Al-Sayyid signers are embedded in several diverse language communities. Hearing signers communicate in both sign language and the local spoken Arabic dialect. Literacy in Hebrew and/or Arabic is increasingly common. Additionally, most men and increasing numbers of women regularly communicate in Hebrew for work, administrative or medical matters. The majority of deaf signers are, or have been, members of student signing communities at school and boarding school outside the village, where communication between students was mostly based on Israeli Sign Language (ISL). Since people regularly move between languages, code switching and mixing are common. Deaf signers may code switch or blend ISL and ABSL, while many hearing signers often switch between speech and 
sign, use signed Arabic, or voice over their own or other people's signing in mixed-group interactions.

Neighboring Al-Sayyid are two villages inhabited by two groups of the Qderat Bedouin that exhibit comparable rates of deafness and hearing signers (Kisch 2007 and this volume). Here too, shared signing communities have emerged. The first Qderat deaf descendants are coevals of the first deaf Al-Sayyid siblings and their deaf descendants started attending the same schools around the same time. ${ }^{10}$ Despite the fact that they can easily demonstrate several obvious lexical variations, the signing of the Al-Sayyid and Qderat is mutually intelligible and they do not consider their signing to constitute separate languages. Moreover, comparable lexical variation exists within the Al-Sayyid community. Both hearing and deaf people often refer to it as 'our language'.

Many lexicalised signs bear evidence for the historical depth of ABSL. For instance, the sign for 'adult woman' or 'mother' is based on the iconic representation of a form of veiling no longer practised. Similarly, the sign for 'butter' derives from the iconic representation of the churning of milk, rarely seen nowadays, for in most households butter is no longer domestically produced (Kisch 2008). The compound place name COW-THERE (pointing to the actual north), referring to the region where some families took their cattle to graze in spring, is still used by some to sign 'Tel-Aviv' (or more generally to indicate the country's central urban agglomeration).

ABSL is used in diverse settings and communication contexts, from casual conversation to resolving disputes and storytelling, and from transactions in local garages or grocery stores to the simultaneous home translation of broadcasted news and other TV programmes. More formal medical, bureaucratic and legal interactions (in Hebrew or Arabic) are often interpreted into ABSL by accompanying relatives. State funded interpreting services (ISL/ Hebrew) are also increasingly employed in such formal settings. Even within the village, ABSL is no longer used exclusively. Like schools outside the village, Al-Sayyid schools take a Total Communication approach to deaf education, based on ISL. Al-Sayyid deaf staff members (along with the nonAl-Sayyid teachers of the deaf) insist that the language of instruction should be exclusively ISL. However, hearing Al-Sayyid staff and peers (lacking command of ISL) regularly use ABSL to communicate with their relatives on school grounds.

Signing is not restricted to deaf signers; in fact hearing signers outnumber deaf signers. Roughly a third of the hearing population is observed to sign regularly. Whereas there are 130 deaf signers, there are more than 700 hearing signers, even when only the most immediate signing relatives are included. 


\section{Shifra Kisch}

This minimum estimate is based on a sample survey comprised of only those hearing signers, such as siblings (and half-siblings), partners and children of deaf individuals, that were regarded by other household members, including at least one deaf relative, as competent signers. Yet (unquantified) ethnographic data indicates there are many more hearing signers than this partial survey suggests, because there is a significant number of hearing signers who have no immediate deaf relatives.

However, the relative ease of communication between deaf and hearing, as well as the status of signed communication, does not solely depend on the number of fluent hearing signers. Indeed, hearing Al-Sayyid use the local sign language with varying degrees of proficiency. Some use local signs only to accompany spoken Arabic. But, even those least proficient often demonstrate awareness of the pragmatics of signed communication and can discuss practical matters fairly easily, preferring translation for more intensive interaction; skilled signers can readily be found to mediate, translate or tutor others in improving their signing skills. Largely due to this widespread awareness and experience of the viability of signed communication, deafness in Al-Sayyid does not make for social marginalization or isolation; deaf people are not categorically shunned or stigmatised. Rather than being considered a defect or disability, deafness is commonly perceived as a condition requiring the use of signed communication.

As manifested in both common attitudes and practices, this relatively inclusive social reality is grounded in daily experiences, that contest the disablement of deafness. There is no evidence that additional characteristics are perceived as inherently attached to deafness, nor are certain social roles or activities reserved for deaf people. The absence of local myths to account for deafness discloses the common perception of deafness as a form of human variation that does not require explaining or fixing. Thus, attempts to reduce the rates of deafness by means of genetic testing and counselling were not received without controversy; compliance to the program was low (Kisch 2004). Likewise, most (hearing) parents were initially reluctant to respond to the active promotion of cochlear implants (CIs). However, over the last five years, after parents were convinced it would increase their children's opportunities, as many as $14 \mathrm{Al}$-Sayyid children have been implanted.

The status of deaf people is gradually being eroded by the differential and restricted structure of their opportunities. The education available for hearing Negev Bedouin is rather dismal, but deaf education has fared worse. While hearing children have been able to attend school since the late 1960s, deaf Al-Sayyid children started attending a Hebrew school for the deaf in Beersheba only in the early 1980s. Later, some of the male deaf students could 
also continue on to receive vocational training at a more distant boarding school. Only in the 1990s were the first classes for deaf students opened in Arabic schools, and in 2004, the first kindergarten (followed by elementary school classes) opened in Al-Sayyid. Out of the total of 134 deaf descendants among all generations of Al-Sayyid, only 14 have never had any form of schooling, and deaf people attend school for longer on average compared to their hearing peers. Nonetheless, literacy among most deaf students remains poor. The separate and mostly inferior schooling available for deaf students presents the most obvious structural disparity between deaf and hearing Al-Sayyid. This schooling has not been designed to prepare students for matriculation certificates, and so higher education has not been available to deaf students. Their options are limited to a few vocational training programmes.

During the adult lives of the first deaf descendants, farming and animal husbandry - the previously dominant sources of livelihood - became severely restricted and income increasingly depended on wage labour. Bedouin men generally inhabit the unskilled, lower strata of the Israeli labour market, with unemployment rates among the highest in the country. Among the Al-Sayyid, sources of income are diverse, including jobs as watchmen, mechanics, tractor or truck drivers, or seasonal agricultural or construction workers. Many commute daily or weekly to work all over the country. There are small business owners and over a dozen hearing young men who have studied abroad in medicine, dentistry, pharmacology and law. Though there is a growing number of both male and female teachers, Bedouin women are otherwise rarely involved in paid labour. Among young deaf Al-Sayyid women however, the rate of employment as trained and untrained educational staff at the local schools is slightly higher than that of their hearing counterparts. With poor literacy but relative ease of access to a limited number of vocational courses, most deaf men and women occupy the middle to lower range of occupations. The recent recession has left many hearing and most deaf men unemployed and dependent on welfare benefits with occasional informal day work.

Over 30 years of separate deaf education (and consequent differential work opportunities) have reduced the social space shared by deaf and hearing Al-Sayyid and transformed deaf people's social networks. This has contributed to the emergence of deaf sociality associated with the use of ISL, as well as a preference for deaf-deaf marriages. In 2004, the marriage of a deaf Al-Sayyid woman to her (non Al-Sayyid Bedouin) classmate constituted the first deaf-deaf marriage among the Al-Sayyid. Since then, half of the marriages of deaf Al-Sayyid women have been with deaf partners almost 


\section{Shifra Kisch}

exclusively from outside the community. Deaf-deaf marriages were initially received with some apprehension, as many deaf and hearing considered the familiar arrangement (the marriage of a deaf and hearing signer) advantageous. Besides, family members and deaf candidates often lacked the social networks to arrange deaf-deaf marriages. This illustrates that whereas the sociolinguistic space shared by deaf and hearing Al-Sayyid is generally accommodating for deaf people, established structures and practices may - as any social configuration elsewhere - both facilitate and restrict (deaf) people. ${ }^{11}$

Since 2005, a team of four linguists ${ }^{12}$ has published extensively on the linguistic structure of ABSL. From the late 1990s the Al-Sayyid have received occasional media attention from regional, national and international media. Publicity among the general public has also increased with two documentaries and the publication of a popular science book. ${ }^{13}$

\section{Notes}

1. Obtaining accurate and reliable demographic data is obstructed by the fact that unrecognised (or newly-recognised) settlements are only very partly included in data published by the Israel Central Bureau of Statistics (ICBS). Additionally, The Al-Sayyid now reside under different municipal jurisdictions, and some still reside outside the jurisdiction of any local or regional council.

2. Scott et al $(1995,1998)$. These publications are also often quoted to state that all deaf individuals are "descendants of two of the five adult sons of the founder" (Scott 1995:965). However, this conclusion was based on partial data collected at the initial phase of the genetic study. Also the use of the term tribe to refer to the Al-Sayyid, used by Scott et al. and in several other publication, is inaccurate.

3. Kisch 2000; 2004; 2008. Last updated survey conducted in the summer of 2011 listing 130 congenitally deaf individuals (Kisch, this volume). Higher rates noted for the Al-Sayyid case are mostly based on (over)estimations of the number of deaf individuals or outdated figures for the total population.

4. Based on my last survey of the total deaf population (rather than a sample) in summer 2011.

5. The smaller north-eastern part of the village is contiguous to one of the seven Bedouin state established townships, and has access to limited municipal utilities. It is no longer part of the Al-Sayyid village as defined in the state recognition plan. 
6. It has been in the process for several years now, but a master plan for the village is still in preparation and negotiation. An approved master plan is required for the provision of many services and building permits. Connection to running water supply and the paving of several asphalt roads are underway.

7. With the prospect of full recognition and lowered risk of demolition, these houses were built by several businesses owners (such as a garage or construction company) and a few young dentists and lawyers.

8. Several Israeli and Jordanian channels offer limited but regular on-screen sign language interpreting, to ISL and LIU respectively.

9. The Negev Bedouin Statistical Data Book, No. 3. 2010; The Galilee Society 2010.

10. For over 30 years deaf Qderat and Al-Sayyid students have had regular contact; together they made up a third of the deaf students at the relevant elementary schools (Kisch 2007; Kisch, this volume).

11. Kisch 2007; 2008.

12. Sandler et al 2005 and other publications by these authors.

13. For review of this book see Kisch 2009.

\section{References}

Kisch, S.

2000 'Deaf discourse': the social construction of deafness in a Bedouin community. Department of Sociology and Anthropology, Tel Aviv University, unpublished MA. [in Hebrew].

Kisch, S.

2004 Negotiating (genetic) Deafness in a Bedouin Community. In Vickrey, J. \& van Cleve (ed.): Genetics, disability, and deafness, 148-173. Washington DC: Gallaudet University Press.

Kisch, S.

2007 Disablement Gender and Deafhood among the Negev Arab Bedouin. Disability Studies Quarterly 27(4).

Kisch, S.

2008 The Social Construction of Deafness in a Bedouin Community in the Negev. Medical Anthropology 27(3). 283-313.

Kisch, S.

2009 Essay Book Review of Talking Hands: What Sign Language Reveals About the Mind. By Margalit Fox. Sign Language \& Linguistics 12(2). 203-210. 
Sandler W., I. Meir, C. Padden \& M. Aronoff

2005 The emergence of grammar in a new sign language, Proceedings of the National Academy of Sciences of the United States of America 102(7). 2661-2665.

Scott, D. A., R. Carmi, K. Elbedour, G. M. Duyk, E. M. Stone \& V. C. Sheffield.

1995 Nonsyndromic Autosomal Recessive Deafness is Linked to the DFNB1 Locus in a Large Inbred Bedouin Family from Israel. American Journal of Human Genetics 57. 965-968.

Scott D. A., M. L. Kraft, R. Carmi, A. Ramesh, K. Elbedour, Y. Yairi, C.R. Srikumari Srisailapathy, S. S. Rosengren, A. F. Markham, R.F. Mueller, N. J. Lench, G. Van Camp, R. J. H. Smith \& V. C. Sheffield.

1998 Identification of mutations in the connexin 26 gene that cause autosomal recessive nonsyndromic hearing loss. Human Mutation, 11 (5). 387-394.

The Galilee Society

2011 The Palestinians in Israel, third socioeconomic survey 2010. Shefa-Amr: The Galilee Society.

The Negev Bedouin Statistical Data Book, No. 3.

2010 Center for Bedouin Studies \& Development, Negev Center for Regional Development, Ben Gurion University of the Negev, Beer Sheva. [Hebrew/English] 


\title{
Sociolinguistic sketch of Ban Khor and Ban Khor Sign Language*
}

\author{
Angela M. Nonaka
}

Ban Khor is a small, Theravadan Buddhist, wet-rice agricultural village in the northeastern Issarn region of Thailand. Like other communities in the area, Ban Khor is highly multilingual. Nyoh, alongside Thai, are the primary languages of everyday life, although Lao, Phuthai, and Soe are also used there. Sociolinguistically, however, Ban Khor is different from most other hamlets in the area. Due to a high incidence of hereditary deafness, the community has a significant number of deaf residents, and in less than a century, they and their close interlocutors spontaneously created a new sign language. For decades the language thrived, expanding rapidly and widely in use throughout the community among both deaf and hearing villagers. That trend began to change, however, early in the $21^{\text {st }}$ century as a consequence of sudden and intensive contact with the national sign language and national Deaf community. By the late 2000s dramatic language shift was occurring and in just half a decade, Ban Khor's local sign language has become endangered.

In-depth analysis of the multiple causes, changing processes, and altered practices underlying and impacting sign language shift and endangerment in Ban Khor is the focus of the main paper on BKSL in this volume. That chapter also provides a detailed account of Ban Khor's ethnographic particulars.

Referred to here as 'Ban Khor Sign Language' (BKSL) for purposes of documentary specificity, the manual-visual language indigenous to the community of Ban Khor, Thailand, is locally known as pasa kidd, the 'language of the mute.' As the term itself suggests, Ban Khorians deem BKSL to be a language (pasa) that they recognise to be different from others they have seen-i.e., other village sign languages as well as the national sign language, Thai Sign Language (TSL). Pasa kidd is a local vernacular expression. While it is used and understood by Nyoh speakers, there is some uncertainty among native speakers as to whether kidd is actually a Soe language word. Whatever its etymological origin, pasa kidd is distinct from the Thai language expression pasa bai, which also translates as 'language of the mute.' Due to the spread of Thai in Ban Khor, the local sign language is also sometimes referred to as pasa bai. The label 'Ban Khor Sign Language' is used 
here to distinguish the village sign language used in Ban Khor from other manual-visual languages found in other villages in Thailand, sign languages that are also referred to locally as the 'language of the mute.'

Ban Khor Sign Language exhibits the traditional subsystems of natural language, including: phonology, morphology, syntax, and semantics, and boasts a rich lexicon (Nonaka 2004, 2007, 2010). As a full-fledged human language, Ban Khor Sign Language also evinces the linguistic unit of 'discourse,' which includes the many genres, registers, and speech acts of a given language as used in conversational interaction by native speakers/ signers. This is certainly true of Ban Khor Sign Language, which evidences a robust range of discourse forms (Nonaka 2007, 2004). A wide range of speech acts in BKSL have been observed and recorded, including: greetings, questions, answers, rhetorical questions, directives, complaints, compliments, offers, acceptances, declinations, summons, thanksgivings, apologies, criticisms, approvals, congratulations, accusations, and warnings. In addition, various discourse structures-i.e., linguistic registers, genres, and activities - have been identified in the language, such as: ordinary conversation, politeness, baby talk, narrative, humor, gossip, quarrels, teasing, language play, interpretation of texts, and cultural meta-commentary.

The village sign language in Ban Khor developed at some point in the early decades of the $20^{\text {th }}$ century. Documentation of the language, however, did not begin in earnest until the start of this millennium. Thus, by the time formal study of the language commenced, BKSL was already a full-blown language. This fact is evidenced by the robust presence of diverse discourse forms and discursive structures and strategies that underscore the viability of BKSL as a full-fledged language of complex reasoning.

The descriptions and analyses of Ban Khor and Ban Khor Sign Language in this volume derive from one of the longest and most extensive continuous studies of a village and its local sign language. Generated through long-term, in-depth, four-fields anthropological research, the Ban Khor data corpus spans well over a decade (1996-2012) and includes hundreds of hours of video recordings of BKSL in situ, in the context of everyday village life. The linguistic data ranges from formal elicitation experiments to natural conversations and was collected from speakers/signers ranging in age from infants to nonagenarians. Formal elicitations were filmed individually, in dyads and in groups. The BKSL data set of natural conversation is especially rich, capturing quotidian talk across multiple domains of everyday life (e.g., at home, at work, at temple). Its centerpiece is a sub-corpus of language socialization data consisting of 11 consecutive months of ethnographic 
focal-follow study of five children (ages 18 hours - 3 years) acquiring Ban Khor Sign Language. In addition to robust linguistic data, the Ban Khor corpus also contains extensive, diachronic demographic information about individuals and their social networks; periodically updated historical and politico-economic data about the community; as well a various mappings of the village.

The scope and duration of the Ban Khor research project are analytically valuable, providing an unusually detailed social context for undertaking diachronic study of a village sign language. Since both linguistic and anthropological data were collected simultaneously at intervals for 10-15 years, the Ban Khor case study is an unusually rich corpus for investigating issues of language change. Reflective of major alterations in the local language ecology, the nature and direction of linguistic change in BKSL has changed dramatically within the last decade. After decades of expansion, BKSL is now contracting, a process that is unlikely to abate. While studies of other village sign languages are expanding understanding of processes of language emergence, it is hoped that examination of Ban Khor and BKSL will shed light on the causes and consequences of village sign language endangerment.

\section{*Acknowledgments}

I am grateful to the following individuals and organizations for their support of my research: Vien Champa, Toni Comer, Connie de Vos, Chettah Madminggao, Nutjaree Madminggao, Chalita Mann, Ronda Rowe, Nipha Sukhwan, Tony Wright, Kate Mesh, Dr. Ulrike Zeshan, Ban Khor Public Health Center, National Science Foundation, Wenner-Gren Foundation, IIE Fulbright, Thai-US Educational Foundation Endangered Language Fund, Explorers Club, University of Texas, UCLA Department of Anthropology, UCLA ISOP (International Studies and Overseas Programs). Last but never least, I thank the community of Ban Khor.

\section{References}

Nonaka, A. M.

2004 The forgotten endangered languages: Lessons on the importance of remembering from Thailand's Ban Khor Sign Language. Language in Society 33. 737-767. 
Nonaka, A. M.

2007 Emergence of an indigenous sign language and a speech/ sign community in Ban Khor, Thailand. Unpublished Ph.D. dissertation, UCLA Department of Anthropology.

Nonaka, A. M.

2010 Interrogatives in Ban Khor Sign Language: A preliminary description. In Mathur, G. \& Napoli, D.J. (eds.) Deaf around the world: The impact of language, 194-220. New York: Oxford University Press. 


\section{Chican Sign Language: A sociolinguistic sketch}

\section{Cesar Ernesto Escobedo Delgado}

Chican Sign Language is a village sign language used in Chican, Mexico. The village of Chican has a population of 720 , most of whom are ethnically Mayan. It is located in Tixméhuac Municipality in the State of Yucatan near Merida Capital. ${ }^{1}$

Deafness in Chican goes back at least three generations. Currently, Chican has 17 deaf signers; eight are female and nine are male. The signers range from seven to 78 years of age, with all age ranges represented. Two of the deaf villagers are married to each other and have two deaf sons. Six of them have hearing spouses, and a further six are single. The vast majority of the deaf villagers have the surname Colli Colli. Some deaf villagers have married hearing spouses from places outside Chican, such as Teabo and Merida. These spouses are not native signers, but acquire Chican Sign Language and Mayan gestures as additional languages. There are also 21 CODAS (Hearing Children of Deaf Adults) in the village.

Due to the unusually high incidence of deafness in Chican, all deaf villagers and a large number of hearing villagers use Chican Sign Language. The hearing villagers also speak Spanish and/or Mayan, and a few also know English because it is taught at government-run secondary schools. People who live in Chican generally have positive attitudes about signing. In 2011, a total of 547 people in Chican were surveyed, of which 121 (22\%) were fluent in sign language, 211 (38\%) had some competence, and $215(39 \%)$ had no signing skills.

Figure 1 shows the layout of Chican, including the location of deaf people's homes (circled). There are three clusters of households containing deaf members, and the largest of these clusters comprises five households. 


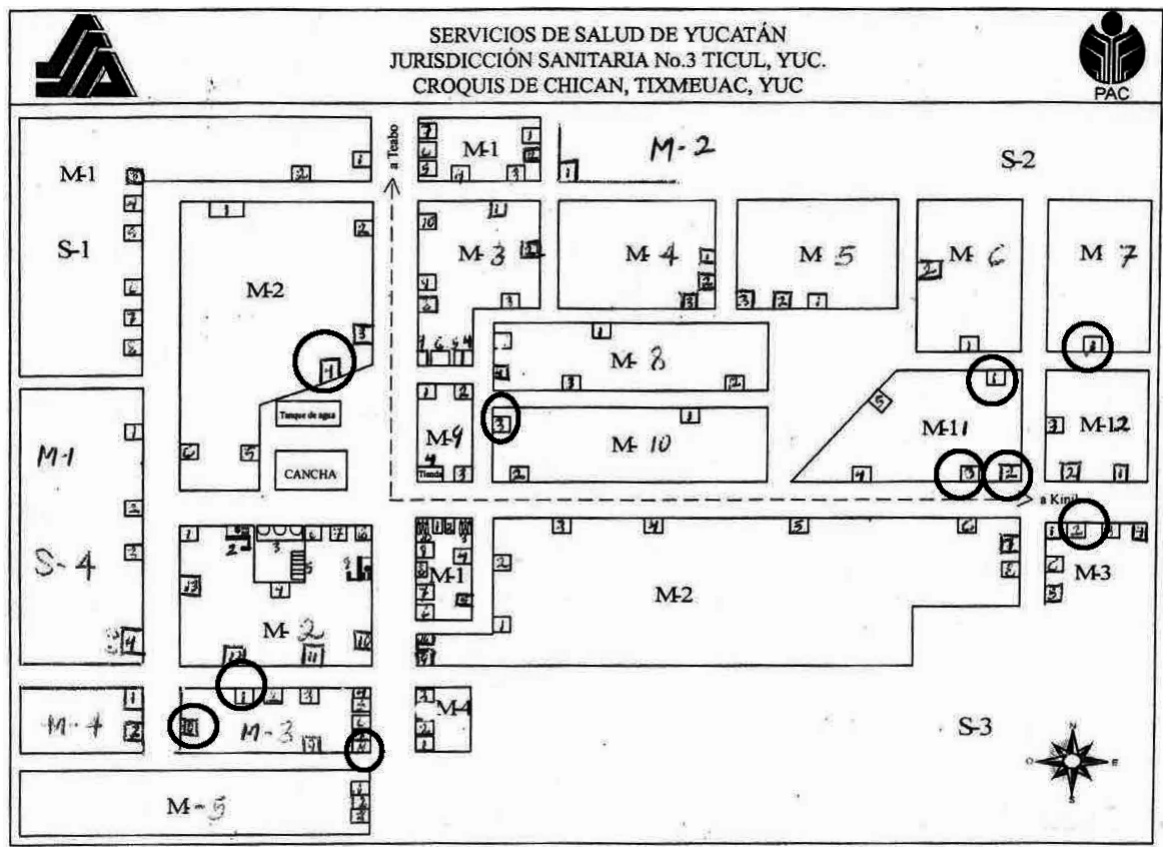

Figure 1. Map of Chican.

Travel between Chican and the nearby towns of Merida, Tekax, Peto, Teabo, and Oxkutzcabo is available by bus, van or car. Villagers commonly travel to these places to shop. Within the village itself, there is a doctor's clinic and health care centre, a yogurt factory, and a local government office with voting facilities.

Crafts and skills which are particularly central to the village's culture include hammock-making, tortilla-making, sewing, spinning, building straw houses, and farm work. Agriculturally, the village relies on producing cows, chickens, and the achiote shrub, an inedible plant whose seeds are valuable for their use as food colourants. Deaf villagers may be employed as cleaners (especially women); builders; bricklayers; artisans; woodcutters; farm workers; vendors selling sodas, crisps, confectionary, fruits and vegetables; and makers of tortillas, hammocks, and clothes (again, women especially). The hearing villagers tend to work in similar capacities but some hold professional positions, including a professor and an accountant. Five hearing villagers have university degrees. Several villagers travel outside Chican for work, to places such as Merida.

In terms of religious culture, Chican is predominantly Catholic with a Presbyterian minority. Religiously, deafness is viewed as a normal phenomenon 
and does not have negative associations as it does in some other cultures; medical explanations for deafness are accepted by the villagers. Interpreters or 'companions' commonly sit next to deaf villagers in church to translate the sermons. ${ }^{2}$

Special education is not available in the village, and at the village's three schools, the teachers do not use sign language. There are currently only four deaf villagers of school age. Chican has one preschool; one primary school, which three of the deaf children currently attend; and one secondary school, which the fourth attends. All three schools run on a matutinal schedule. Outside the village, in Tekax de Alvaro Obregón, there is a government-run special school for deaf pupils as well as those with physical and/or learning disabilities. This school relies on oral, sound-based and total communication methods, with very little emphasis on Mexican Sign Language (LSM). However, one teacher at this school has expressed an interest in learning Chican Sign Language and is eagerly anticipating the publication of a sign language dictionary.

Though there are several Chican Sign Language interpreters who translate for health care appointments, political campaigns and elections, they are not trained or qualified. But most interpreters in Mexico use LSM and do not know Chican Sign Language, so Deaf people from Chican will often ask a hearing villager to interpret for them instead.

Technology available in the village includes several television channels as well as local and mobile phone networks. However, only hearing villagers use mobile phones, and their efficacy is inadequate anyway due to weak signals in the area. On-screen LSM interpreters are sometimes shown on news programmes, but televisions are uncommon, as are DVD players. Internet facilities do not exist in Chican, but the hearing villagers travel to Tekak for access. Deaf villagers tend not to use the internet or mobiles due to their limited proficiency in Spanish and Mayan. Computers are a rare sight in the village, apart from in the schools. Webcams are also scarce.

In terms of assistive technology, hearing aids are widespread, due to donations by many charities. Cochlear implants have not yet been used within Chican.

Signers do not generally move out of the village, but they have some contact with signers of other languages, especially LSM users from Merida. The deaf children of the village have been known to learn a few LSM signs (e.g. those for 'airplane' and 'water', as well as the alphabet) from teachers at the special school in Tekax. At one point, Jehovah's Witnesses from Merida wanted to teach LSM to the deaf villagers with the aid of a DVD and a paper copy of the manual LSM alphabet, but the villagers refused, and were 
unable to watch the DVD in any case because that technology is unavailable in Chican. Foreign researchers have also visited the village, but have not attempted to teach their country's sign languages.

\section{Notes}

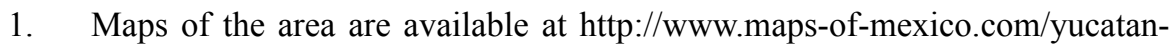
state-mexico/yucatan-state-mexico-map-b2.shtml and http://mexico. pueblosamerica.com/mapas/chican (accessed 03 February 2012)

2. A picture of Chican's Presbyterian Church is available here: http://galeon. com/chican/templo.html (accessed 03 Feburary 2012). 


\section{Kata Kolok: An updated sociolinguistic profile}

\section{Connie de Vos}

Kata Kolok is a sign language used by the deaf and hearing inhabitants of a farmers' village in the North of Bali, in the region of Buleleng. The hearing villagers refer to Bengkala as Desa Kolok - which is Balinese for 'deaf village' - and its sign language as Kata Kolok 'deaf talk'. The deafness in Bengkala is recessive, non-syndromal, and sensorineural and it is caused by a mutation of the gene referred to as DFNB3 or MYO 15a (Friedman et al. 2000). The mutation that causes deafness is widespread throughout the village population, and as a result $2.2 \%$ of the villagers are congenitally deaf, but $17.6 \%$ of the hearing community members also carry the 'deaf' version of the gene (Winata et al. 1995). A reconstruction of the village's lineages reveals that the first person to be affected by this gene was born seven generations ago (Liang et al. 1998). However, it was not until five generations ago that the language was used by a small group of deaf signers and it is this event that marks the emergence of Kata Kolok (de Vos 2012).

According to demographic counts in 2008 Bengkala's population is approximately 2,740 (Astika 2008). A visit to the community in September 2011 has identified 46 deaf signers, spread throughout the dadya 'village clans'. Notably however, 8 deaf individuals have migrated to other parts of Bali, Indonesia, and even Australia for educational, socio-economic, and marital reasons. A map of the current geographical distribution of deaf individuals in the village is presented in Figure 1. Because the central village is small and covers less than a square kilometre, deaf and hearing villagers live in close proximity of one another and frequently interact at food stalls and kiosks throughout the village.

Apart from casual chatting, Kata Kolok is also used in professional, liturgical, and educational settings by both deaf and hearing villagers. It is, for example, used in water pipe maintenance - which is vital to the village's farming activities - and by the village nurse when she tends to deaf villagers. Kata Kolok is also used in child-directed signing between infants and their caregivers where either the infant or the caregiver (or both) are deaf (Marsaja 2008:103). The sign language even surfaces on the rare occasion when a pandetta, a Hindu priest, is possessed by a deaf god during a trance. Since 2007 Kata Kolok has been also been used as a language of instruction in the village's elementary school (Kortschak 2010). 


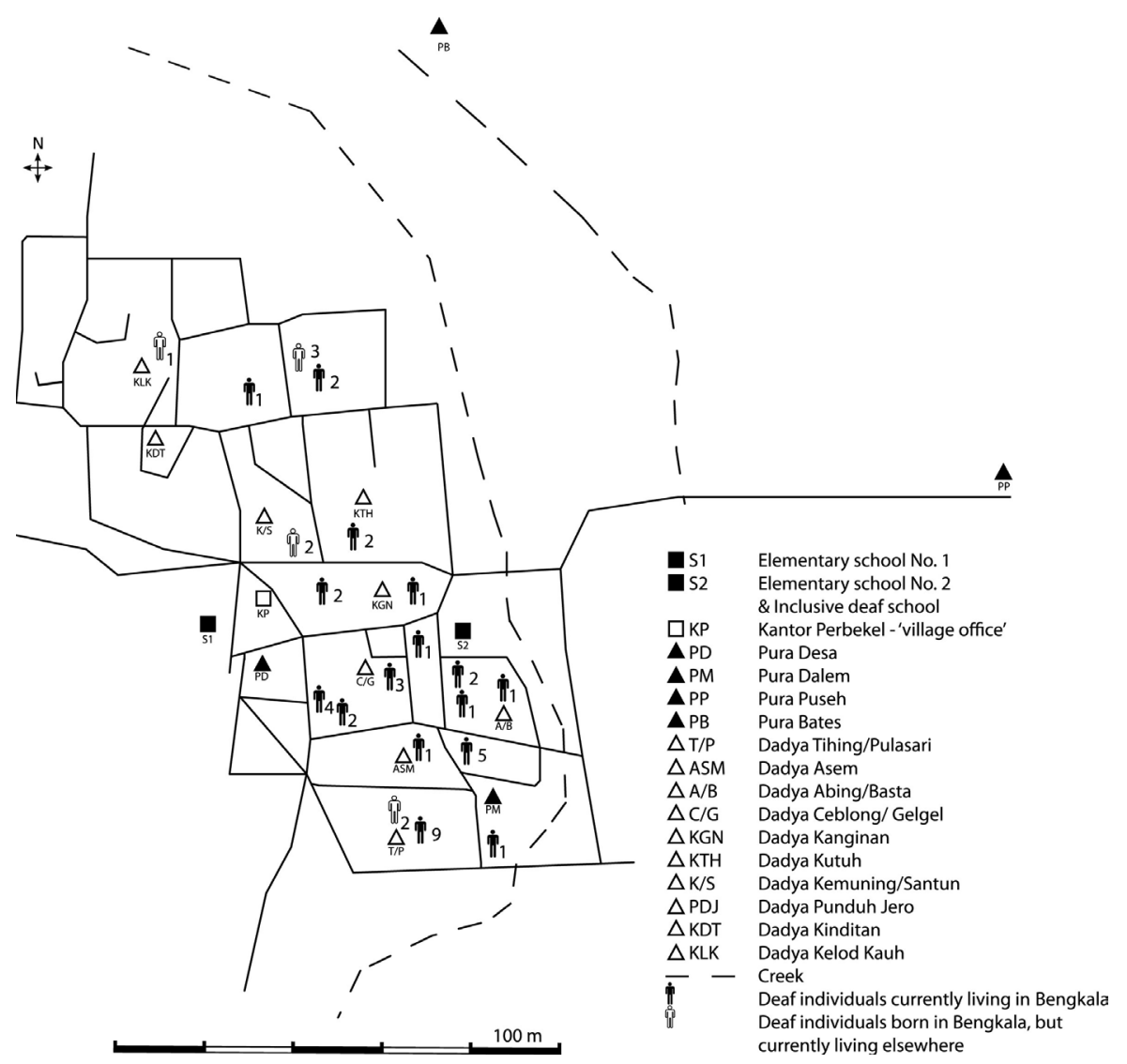

Figure 1. The geographical distribution of deaf individuals in Bengkala in 2011

Deaf villagers use signs to communicate with their hearing relatives, as well as many of their hearing friends and colleagues, and a survey conducted in 2000 has indicated that at least $57 \%$ of Bengkala's hearing population can understand and use Kata Kolok with varying degrees of proficiency (de Vos 2012). As a result, deaf signers from Bengkala do not experience the same social inequalities as many Deaf signers from urban signing communities do. The integration of deaf villagers is also mirrored by the fact that they have equal chances of getting married and similar professional opportunities (Branson et al. 1999). Moreover, many village activities are shared between deaf and hearing villagers. These include the Hindu ceremonies as well as issues pertaining to village security. In these joint activities the deaf villagers are well-integrated into the wider hearing community.

Most of the deaf and hearing villagers earn a living by dry-land farming. Crops are grown in and around the village, including turmeric, ginger, peanuts, 
corn, cassava, bitter cucumber and cashew nuts. In addition, seasonal fruits such as rambutan, guava, mangoes, bananas, and oranges are cultivated. Local businesses include food stalls, construction work, carpentry, transport, and tailoring. Most of the villagers earn a living by farming other people's land, and make less than 10,000 Indonesian rupiah a day (Marsaja 2008). As such, they live in poverty, even by Indonesian standards. The main religion of Bali is Hinduism; all inhabitants of Bengkala are Hindu. Hindu ceremonies play an important role in the lives of the villagers, as they form an occasion to socialise with clan members, prepare and eat special food, and have time away from more onerous duties.

Although deaf individuals in Bengkala do not share the same kind of struggles as deaf individuals outside the deaf village, there are some indications that here, too, a social construction of deafness may be found, which is shared by both deaf and hearing villagers. Most strikingly, all villagers believe in a deaf god who dwells in the village cemetery. Secondly, deaf villagers are referred to in a different yet positive way that distinguishes them from hearing villagers. In general, Balinese people are called by their first name, which is determined by the birth order within a family. For example, the fourth child in a family is called Ketut, independent of gender. When hearing villagers talk about deaf individuals in spoken Balinese, they preface the person's given name with the word kolok. For example, a deaf man called Getar would be referred to as kolok Getar. Kolok is a title meaning 'deaf,' and although the use of the word kolok is perceived of as stigmatising in other parts of Bali, this does not seem to be the case in Bengkala. In fact, the kolok men are often characterised as particularly strong yet sensitive, and dominate the village's civil defence brigade for this reason (see also Marsaja 2008:72). The deaf men are also responsible for burying the dead at the village's cemetery.

A third piece of evidence for the social construction of deafness in Bengkala stems from the existence of a 'Deaf Alliance' (Marsaja 2008:73). Deaf men, sometimes joined by deaf women, will gather in a ritual to slaughter an animal and prepare lawar (chopped meat and vegetables with spices) followed by the sharing of tuak (palm wine). At these deaf gatherings a deaf dance is often performed, called the janger kolok (also see Marsaja 2008:75). At the start of the ritual, the deaf men sit in a semicircle. The kolok leader taps a beat on an empty box, while the other men produce a visual rhythm by arbitrary, waving hand movements. This part of the dance is also accompanied by regular high-pitched yells from the deaf signers. After several minutes, one of the men will get up, drink a glass of tuak, and enter the semicircle; he then performs a dance that mimics martial arts. The janger kolok is also 
performed at more formal occasions, such as Indonesia's annual Independence Day on 17 August. On these occasions no alcohol is involved, the kolok wear special uniforms, and a few women may join the dance. See Figure 2 below for an impression of such a formal performance of the janger kolok.

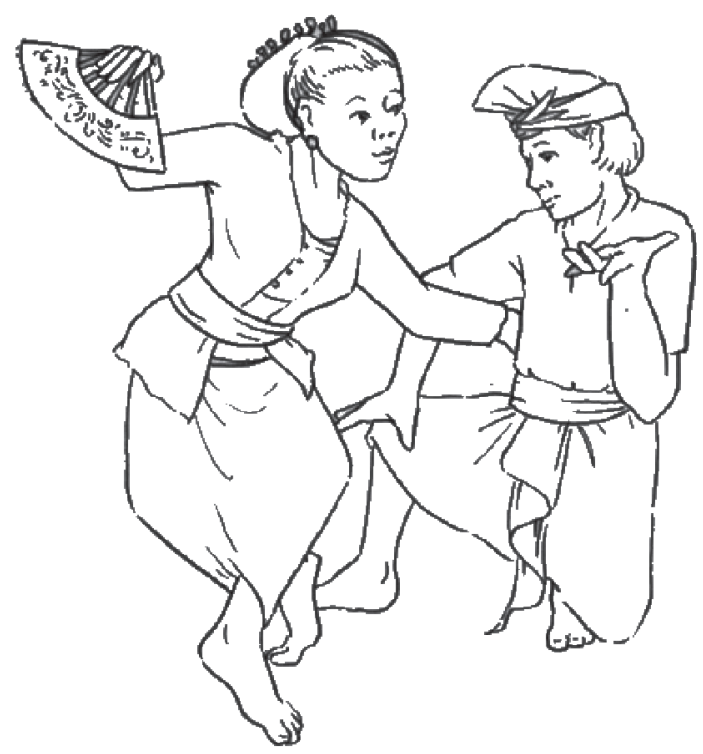

Figure 2. The janger kolok, a deaf dance

I would like to conclude this sociolinguistic sketch by describing the marked increase of national and international contacts with the Kata Kolok signing community in recent years. Since 2009, two volunteers have been involved at the deaf unit that was set up in the village's elementary school. This project has been funded by a Dutch foundation called Vrienden van Effatha, and these contacts have led to many visits by (mostly) Dutch tourists. Furthermore, following a publication supported by the World Bank (Kortschak 2010), Bengkala has attracted considerably media attention from regional, national, and international newspapers and television programmes. Kata Kolok signers even feature in the jingle that precedes the daily news on tvOne - one of Indonesia's national broadcasting agencies. Bengkala has since become a famous attraction, and there are few Balinese people who have not heard of it (cf. de Vos 2012). In response to these changes, the Deaf Alliance has been registered with the regional government since early 2012 
and now comprises a group of both deaf and hearing community members that advocate the interests of deaf villagers and their hearing relatives (de Vos \& Palfreyman, forthcoming). This group evaluates requests for media coverage and research projects and arranges interpreting when necessary. The Deaf Alliance, together with a local artist as well as two deaf mothers, has also begun educating deaf villagers through the production of a pedagogical dictionary of Kata Kolok (de Vos \& Palfreyman forthcoming). While it is uncertain how this increased contact with the outside world may play out in future, it is clear that the Kata Kolok signing community is sufficiently empowered to manage the changes at hand.

\section{References}

Astika, I. M.

2008 Data monografi desa dan kelurahan. Buleleng: Kantor Pemberdayaan Masyarakat Desa Kabupaten Buleleng.

Branson, J., D. Miller \& I.G. Marsaja

1999 Sign languages as a natural part of the linguistic mosaic: The impact of deaf people on discourse forms in North Bali, Indonesia. In: Lucas, C. (ed.), Sociolinguistics in Deaf Communities, 109148. Washington, D.C.: Gallaudet University Press.

De Vos, C.

2012 Sign-Spatiality in Kata Kolok: how a village sign language inscribes its signing space. $\mathrm{PhD}$ dissertation. Max Planck Institute for Psycholinguistics, Nijmegen.

De Vos, C. \& N. Palfreyman.

forthcoming [Review of the book Deaf Around the World: the impact of Language, edited by Mathur \& Napoli (eds.)]. Journal of Linguistics.

Friedman, T. B., J. T. Hinnant, R. A. Fridell, E. R. Wilcox, Y. Raphael, \& S. A. Camper

2000 DFNB3 families and Shaker-2 mice: mutations in an unconventional myosin, myo 15. Advances in Oto-Rhino-Laryngology 56. 131-144.

Kortschak, I.

2010 Where everyone speaks deaf talk. Invisible people: Poverty and empowerment in Indonesia, 76-89. Jakarta: PNPM Mandiri. 
Liang, Y., A. Wang, F. J. Probst, I. N. Arhya, T. D. Barber, K. S. Chen, D. Deshmukh, et al.

1998 Genetic mapping refines DFNB3 to $17 \mathrm{p} 11.2$, suggests multiple alleles of DFNB3, and supports homology to the mouse model shaker-2. American Journal of Human Genetics 62(4). 904-915.

Marsaja, I. G.

2008 Desa Kolok - A Deaf Village and its Sign Language in Bali, Indonesia. Nijmegen: Ishara Press.

Winata, S., I. N. Arhya, S. Moeljopawiro, J. T. Hinnant, Y. Liang, T. B. Friedman, \& J. J. Asher

1995 Congenital Non-Syndromal Autosomal Recessive Deafness in Bengkala, an Isolated Balinese Village. Journal of Medical Genetics 32(5). 336-343. 


\title{
Sociolinguistic sketch of Konchri Sain
}

\author{
Keren Cumberbatch
}

Konchri Sain is the minority sign language in Jamaica. Konchri Sain originated in the agricultural village of Top Hill and spread to the immediately neighbouring communities. Top Hill is approximately $110 \mathrm{~km}$ from Kingston, the capital of Jamaica. Like some other postcolonial territories, Jamaica has an official language, which is the language of its former coloniser, existing alongside a nonofficial Creole lexified by that language. English, is the official language of Jamaica. Patwa, also called Jamaican Creole, is an English-lexified Creole. It is widely believed in Jamaica that the parish of St. Elizabeth has the most basilectal form of Patwa. Interestingly, it is in this same parish that one finds Konchri Sain, the rural sign language indigenous to Jamaica. Moreover, the language attitudes of the hearing toward Patwa are the same of the Deaf toward Konchri Sain. These indigenous Caribbean languages are treated as primitive, undesirable and indicative of low socioeconomic status of its users. However, while Patwa continues to be used by the masses despite the negative language attitudes, Konchri Sain faces its deathbed. There is a change of tides approaching as language rights advocates lobby for official status for Patwa and public opinion is swinging in its favour and the research into Konchri Sain creates awareness of its status as a heritage language.

Besides the spoken languages, English and Patwa, Konchri Sain has other signed communication systems in its environment. Signed English, American Sign Language and Jamaican Sign Language are all used by the Deaf in Top Hill and surrounding communities. American Sign Language and Jamaican Sign Language are mutually intelligible and it can be argued that Jamaican Sign Language is a dialect of American Sign Language. Signed English and American Sign Language were introduced to the area in 1975 when American Mennonite missionaries established a school there. Prior to having a school in Top Hill, children were usually sent to Kingston for education. Those children experienced additive bilingualism. They used Signed English and American Signed English at school and Konchri Sain when they returned home. Regrettably, the school in Top Hill practised subtractive bilingualism. The use of Konchri Sain at school even outside of the classroom was strongly discouraged. Some elderly persons in Top Hill reported being punished for using Konchri Sain in the playground. The school staff 
also instructed parents that they should not use Konchri Sain at home as this was not in the best interest of the children and would impede their learning. Thus began the death of Konchri Sain.

Up to this point, Konchri Sain and Patwa were the main languages of the area. The deaf used Konchri Sain and the hearing used Patwa and Konchri Sain. Being a hearing person who was fluent in both a spoken and a signed language was the norm. Due to a high incidence of deafness in the area, deaf persons were usually born into families with deaf relatives and no social stigmas were attached to deafness. Several deaf informants reported that hearing caregivers like parents, grandparents and aunts, taught them Konchri Sain. This has changed. Now the only monolingual users of Konchri Sain are elderly. Since Signed English and American Sign Language were the languages of instruction at the school, they became the languages of daily life in the Deaf community. Interaction with other Deaf across the island and migration to urban areas increased. Exposure to Jamaican Sign Language being used by urban Deaf led to a further loss of prestige for Konchri Sain. Jamaican Sign Language was seen as having more value. Today, the average Deaf adult uses Jamaican Sign Language as his primary means of communication. Deaf Church services are conducted in Signed English with interpreting provided for monolingual Konchri Sain users. Most middle-aged deaf adults are bilingual in Konchri Sain and Jamaican Sign Language. When asked about their knowledge of Konchri Sain, deaf and hearing young adults often reply that they know a few signs and do not consider themselves users of the language. They learnt the words they know from parents and others in that generation. Konchri Sain is not associated with the young. An aim of research into Konchri Sain structure has been to stimulate awareness of the status of Konchri Sain as a heritage language and foster positive language attitudes towards it in the community. Fortunately, this is happening. There is now an interest among the elderly in passing the language on to the next generation. It is hoped that the younger community members, both deaf and hearing, will become proactive in attaining fluency in Konchri Sain.

Mennonite missionaries are still present in the community working at the school. They use Signed English and American Sign Language. This is also different from most Deaf schools in the island where the primarily Baptist American missionaries have been recalled. This contributed to the evolution of Jamaican Sign Language as there was no longer constant direct input of American Sign Language users into the communication system of the Deaf. 


\title{
Sociolinguistic profile of Inuit Sign Language
}

\author{
Joke Schuit
}

\section{Geography}

Inuit Sign Language, or Inuit Uukturausingit (IUR) in Inuktitut, is a language of Nunavut, Canada's Arctic territory (see Figure 1 below), where $85 \%$ of the population is Inuit. The name Nunavut means 'our land' in Inuktitut, the spoken language of the Inuit. Nunavut is Canada's largest territory, encompassing about 2 million $\mathrm{km}^{2}$ (or 787,000 square miles). About 32,000 people live in 25 communities spread throughout the territory (Census of Canada 2011, available online). Travel to and among Nunavut's communi-

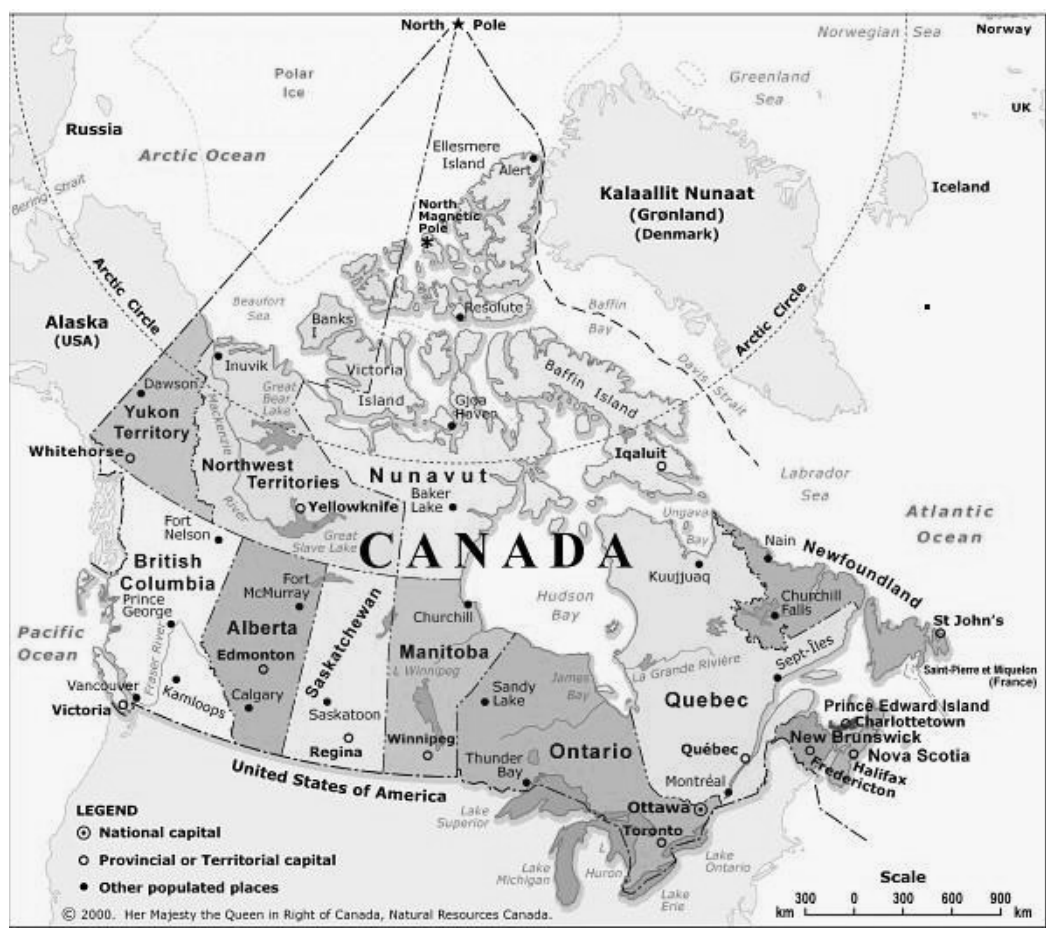

Figure 1. Map of Canada. 
ties is by airplane, as there are no roads connecting them. In Nunavut, transportation can be by car, truck, all-terrain vehicle or snow mobile, although snow mobiles cannot be used in the summer. Flights between the communities are rather expensive, and face-to-face contact among deaf Inuit from different communities is rare. IUR is thus used in quite an extraordinary sociolinguistic setting: less than 40 deaf signers use the language as their sole means of communication, and they live in different locations, spread across an immense area. IUR is not used in a single village community, as are the other sign languages described in this volume. The geographical spread of the sign language is somewhat reminiscent of Plains Indian Sign Language, as was pointed out by an anonymous reviewer. However, Plains Indian SL is mainly used as an alternate sign language by hearing people (Davis 2010). In contrast, IUR is no longer used by hearing people, but signs were previously used by hearing Inuit in communication with people from different dialects (Olsthoorn 2010), and possibly also for trade.

Information about IUR has been gathered in the communities of Baker Lake, Rankin Inlet and Taloyoak (see Figure 2 below). It is possible that deaf Inuit live in all the communities, but whether they use IUR or American Sign Language (ASL) is not known.

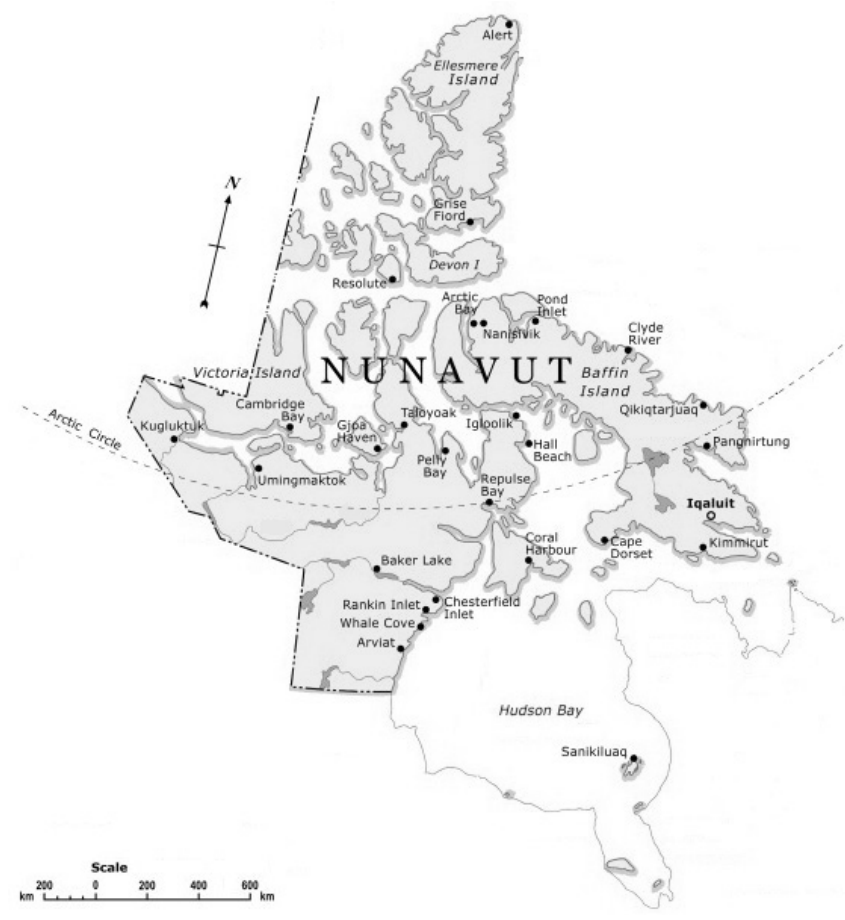

Figure 2. Map of Nunavut. 


\section{History}

Historically, the Inuit were hunter-gatherers, and led a nomadic life (Wachowich 1999). For this reason, MacDougall (2000) suggests that the origins of IUR could be similar to those described for Aboriginal people in North and South America and Australia, whose sign languages developed as alternate communication systems used during hunts, and/or as lingua francas in cases where the people spoke mutually unintelligible languages or dialects (see MacDougall 2000). Indeed, signs were used in Inuit culture as early as the 18th century to communicate among different bands (Olsthoorn 2010). For reasons unknown, a rather high percentage of Inuit were born deaf or became deaf. The signs already existing in Inuit culture probably were used with the deaf children, and evolved into a sign language.

\section{Social situation}

It should be taken into account that the situation of deaf people in southern Canada is quite different from that in Nunavut, and different sign languages are used. In English-speaking parts of Canada, ASL is used, and Frenchspeaking parts use Quebec Sign Language (LSQ). Some elderly deaf people in the Maritime Provinces (New Brunswick, Prince Edward Island and Nova Scotia) still use Maritime Sign Language, a variety descended from British Sign Language, which is nearly extinct (Yoel 2009). However, none of these languages is related to IUR.

The territory of Nunavut is multilingual; most of its inhabitants speak at least two languages: Inuktitut and English. Inuktitut is the traditional language of the Inuit, and has many dialects. English, being one of Canada's official languages, is indicated by almost half of the inhabitants of Nunavut as the language most often used at home (Census of Canada 2006, online).

Deaf Inuit use either ASL or IUR. Those between the ages of 40 and 60 were sent to schools for the deaf in southern, English-speaking Canada. In these schools, various communication systems were used, but all were associated with non-Inuit culture and language. Educated deaf Inuit therefore use ASL, Manually Coded English, or a sign language/system in between. Deaf children nowadays attend school in their home community, with the aid of an ASL/English interpreter. IUR is not acquired by any of these deaf children, and is therefore highly endangered.

IUR is used by deaf Inuit who have had little or no formal education. All signers are over 40. Using statistics, MacDougall (2000) estimates that 
approximately 47 deaf Inuit use IUR as their main means of communication. However, interviews carried out by the author during a fieldwork visit in March 2009, in which the inhabitants of nine communities gave information about deaf people, seem to indicate that this number is too high. If MacDougall were correct, there would have to be 75 deaf people overall in those communities (based on the Census of Canada 2006), but the interview participants suggested there were about 20. MacDougall (2000) also reports that up to $75 \%$ of the hearing population in Baker Lake signs, but to what extent they have mastered IUR is as yet unclear.

There appears to be no negative attitude toward deafness among the Inuit, and especially no social exclusion because of deafness. Although the descriptive project did not focus on social relations, no restrictions regarding marital patterns or employment were observed. Deaf Inuit, whether they use ASL and IUR, seem able to freely choose any occupation, and can be artists, hunters, clerks and general labourers.

The general attitude towards sign language is positive, and many Inuit regard it as a natural, full language, albeit distinguishing between the native sign language (IUR) and the non-native one (ASL). No official interpreters exist for IUR. Often a family member or friend acts as an interpreter when needed.

A few deaf signers acknowledge deafness as a 'connecting factor'. It would be somewhat misguided to equate this to the Deaf identity found amongst Deaf people in urban societies, as no specific characteristics that could be classed as Deaf culture are recognised among deaf Inuit. In addition, IUR signers all identify strongly with the Inuit culture, rather than with Deaf culture. Therefore it would perhaps be more appropriate to refer to a 'deaf connection' in this case. The situation in other parts of Canada is different (see Carbin \& Smith 1996).

In order to determine the status of sign language in their territory, the Nunavut Ministry of Culture, Language, Elders, and Youth (CLEY) set up a focus group in 2006. As a result of the focus group, a lexicology project (the Inuit Sign Language Lexicon Project) was established, as was a project aimed at documenting the history of Nunavut's deaf inhabitants. Both projects were concluded in 2008, and the Minister of CLEY declared in the Legislative Assembly that "the uniqueness and importance of Inuit Sign Language" be recognised (Nunavut Hansard, 16-09-2008, available online). 


\section{References}

Carbin, C. \& D. Smith

1996 Deaf Heritage in Canada: A distinctive, diverse and enduring culture. Toronto: McGraw-Hill Ryerson

Davis, J.E.

2010 Hand Talk. Sign Language among American Indian Nations, Cambridge: Cambridge University Press

MacDougall, J.

2000

'Access to justice for deaf persons in Nunavut: Focus on signed languages.' Report for the Research and Statistics Division, Dept. of Justice, Canada

Olsthoorn, T.

2010 Die Erkundungsreisen der Herrnhuter Missionare nach Labrador (1752-1770). Kommunikation mit Menschen einer nichtschriftlichen Kultur, Hildesheim: Olms

Wachowich, N.

1999 Saqiyuq. Stories from the lives of three Inuit Women. Montréal: McGill-Queen's University Press 


\section{Mardin Sign Language: Signing in a "deaf family"}

\section{Hasan Dikyuva}

Mardin Sign Language (MarSL) is a recently-documented, small-scale sign language used by an extended family in Turkey. MarSL originated in Mardin, a town in south-eastern Turkey close to the Syrian border. Its development, dispersion and user community approximates the situation of other rural community sign languages. Beginning around the 1930s, MarSL developed as a result of genetic deafness in this family, which includes at least four successive generations of deaf individuals (Dikyuva \& Dilsiz 2009, Dikyuva \& Zeshan, in press). The family's name, Dilsiz, means 'deaf' (literally 'tongue/language-less') in Turkish. The name Mardin Sign Language was coined by researchers; the signers themselves refer to their language as dilsizce (Turkish for 'deaf language') or eski işaretler (Turkish for 'old signs').

MarSL is currently used by an estimated 40 people, including both deaf and hearing members of the Dilsiz family. It is mainly used at home and at family gatherings such as weddings. The language is highly endangered and not acquired by children any more.

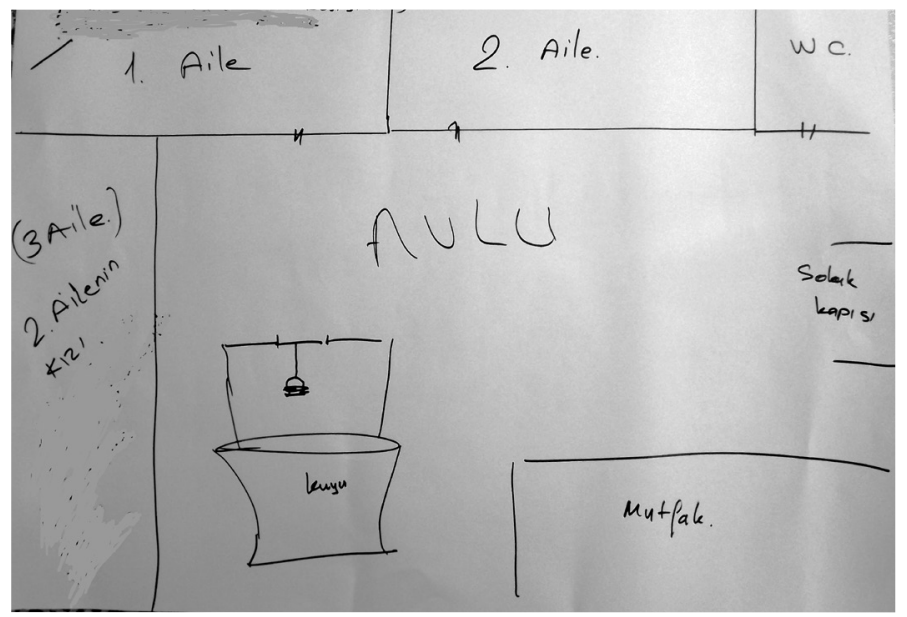

Figure 1. The Dilsiz family compound in Mardin

Figure 1, which was drawn by an elderly MarSL consultant, depict the multifamily compound where the Dilsiz family lived when the sign language first 
developed (the written labels are in Turkish). This is an atrium-style (aulu) building with an open courtyard, as was typical of the area at the time. As Figure 1 illustrates, the compound has a central courtyard around which three related families (aile) live. These three families constituted the core of the MarSL community. Family 1 and family 2 were established by an uncle and his nephew, both of whom had a large number of children of partly overlapping ages. Family 3 consisted of the eldest daughter from family 1, her husband and one child.

Each family's living area opens into the central courtyard, which itself opens onto the street (sokak) to the right of the picture. Everyone in the compound shared the bathroom and kitchen (mutfak) facilities, and the well in the courtyard. Therefore, communal living was the norm, and all hearing people in the compound were fluent in the sign language used by the several deaf family members in the aulu. Later on, several of the children from families 1 and 2 moved out of the compound as adults and established independent homes elsewhere in Mardin.

Figure 2 shows part of the Dilsiz family tree. This is only a small selection of the family; a more extensive diagram created by several consultants during research includes several hundred names. The deaf individuals are marked by grey colour in the diagram. There are both male and female family members among the deaf, though males are in the majority. The two eldest deaf individuals that could be indentified were a brother and sister. Halil and Abdüsselam were the heads of family 1 and family 2 respectively. When their children (mostly born in the 1950s and 1960s) were growing up in the compound, sign language use was already fully established.

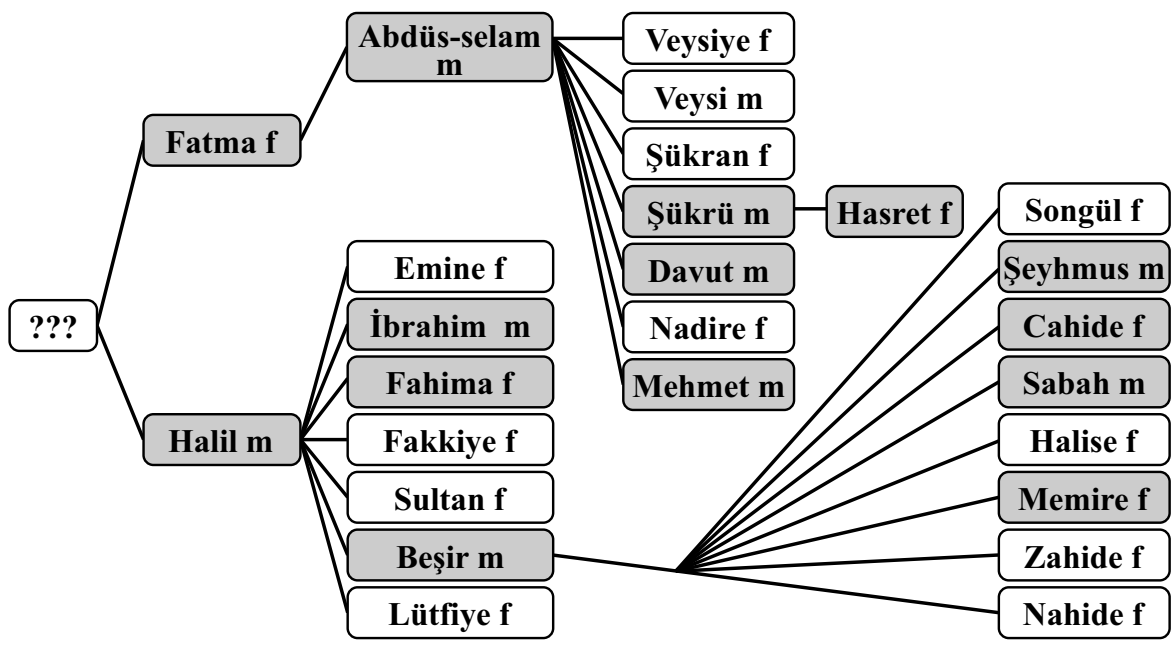

Figure 2. Part of a family tree diagram of the Dilsiz familz 
Figure 3 shows a map of Mardin from a time period around the 1950s, as remembered by MarSL consultants. Mardin has developed and grown rapidly since then, but at the time had a single circular main road. In the area surrounded by the road, one could only travel on foot. The crosses indicate where deaf people lived at the time. Most of them are from the Mardin family, but there were several other deaf individuals as well as another family with several deaf siblings, all of whom participated in signed communication. The dotted lines represent the distance between houses, and the accompanying numbers reveal the walking time in minutes.

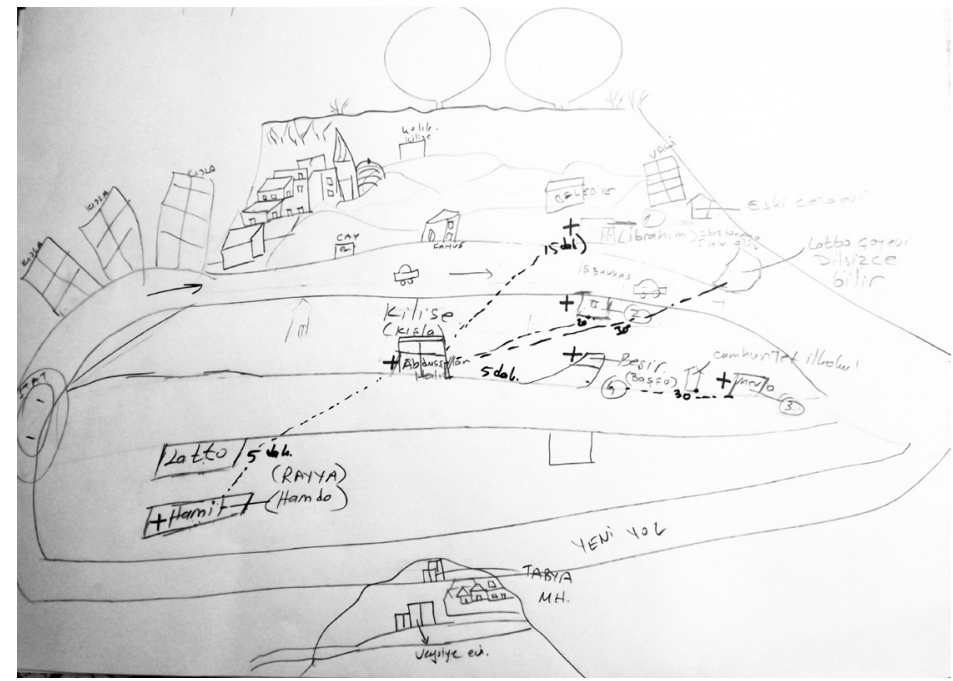

Figure 3. Map of Mardin as represented by MarSL consultants

Due to community dispersal and increasing contact with Turkish Sign Language (TID), MarSL is now on the brink of extinction. Most of the fluent MarSL users are in their 50s and 60s, and the oldest living user is a hearing woman in her 70s. About 25 years ago, family members began moving to Istanbul and Izmir in search of better employment opportunities. The deaf family members then came into contact with the large and well-organised Turkish Sign Language using communities in these cities. Deaf children born since then attended school for the deaf in Istanbul and Izmir, and therefore shifted to using TID instead of MarSL. Today, some family members remain in Mardin, but not any of the deaf individuals.

As the first estimated use of MarSL dates back to the 1930s, this constitutes considerable time depth compared to many other sign languages (cf. Nonaka 2009). From the 1930 s to 1980 s, there is very little evidence of any contact with TID, because Mardin did not have schools, clubs or associations 
of deaf people using TIDD. Most of the deaf children growing up in Mardin never went to school.

Therefore, MarSL has existed in two quite distinct settings: where the language first arose (ca. 1930 - 1985), and where it is in decline (ca. 1985 - present). The former was multilingual with respect to spoken languages, namely Turkish, Kurdish and Arabic. The Dilsiz family's main home language was the local variety of spoken Arabic, and there is evidence of contact between MarSL and spoken Arabic, particularly in the occurrence of mouth movements accompanying some of the signs (see Zeshan et al. (in prep.) on cardinal numerals in MarSL). In the latter setting, all deaf members of the Dilsiz family are either monolingual in TID (the younger members, who go to deaf schools and deaf clubs in the urban deaf community) or bilingual in TID and MarSL (the older members, who maintain social networks through both sign languages). Perhaps surprisingly, MarSL is maintained mainly due to hearing family members, who are 'sign monolingual' in MarSL and do not know TiD because they have little incentive to associate with the urban deaf community (see Lanesman 2012 for a parallel case in Israel). Thus, the deaf family members use MarSL in communication with their hearing relatives, and the monolingual TID signers have a limited ability to do so. Spoken language shift is also evident in the family, as hearing people have been shifting from Arabic to Turkish. Presently only the oldest hearing family members have limited competence in spoken Turkish, while all others have increasingly strong socialisation in Turkish through education, media, work and social life in Istanbul and Izmir.

\section{References:}

Dikyuva, H. \& H. Dilsiz

2009 An extended "deaf" family in Turkey. In Panda, S. (ed.) Sign Languages in Village Communities (Ishara Signed Publications No. 2). Nijmegen: Ishara Press.

Dikyuva, H. \& U. Zeshan

in press Documentation of endangered sign languages - The case of Mardin Sign Language. In: Jones, M. \& S. Ogilvie (ed.): Keeping Languages Alive: Documentation, Pedagogy and Revitalization. Cambridge: Cambridge University Press.

Lanesman, S.

2012 Algerian Jewish Sign Language: Its emergence and survival. MA dissertation, University of Central Lancashire, UK. 
Nonaka, A.

2009 Estimating Size, Scope, and Membership of the Speech/ Sign Communities of Undocumented Indigenous/Village Sign Languages: The Ban Khor Case Study. Language \& Communication 29(3): 210-229.

Zeshan, U., H. Dikyuva, C. E. Escobedo Delgado, S. Panda \& C. de Vos

in prep. Cardinal numerals in village sign languages - Approaching crossmodal typology. 


\title{
Yolngu Sign Language: A sociolinguistic profile
}

\author{
Elaine L. Maypilama and Dany Adone
}

Yolngu Sign language (henceforth YSL) is an indigenous sign language used by both deaf and hearing Yolngu people in Arnhem Land, northern Australia. Both the hearing and deaf population refer to YSL as 'action' in English and as djama gongdhu 'work with hands'. In this study we focus on YSL as used in the Galiwin'ku community on Elcho Island.

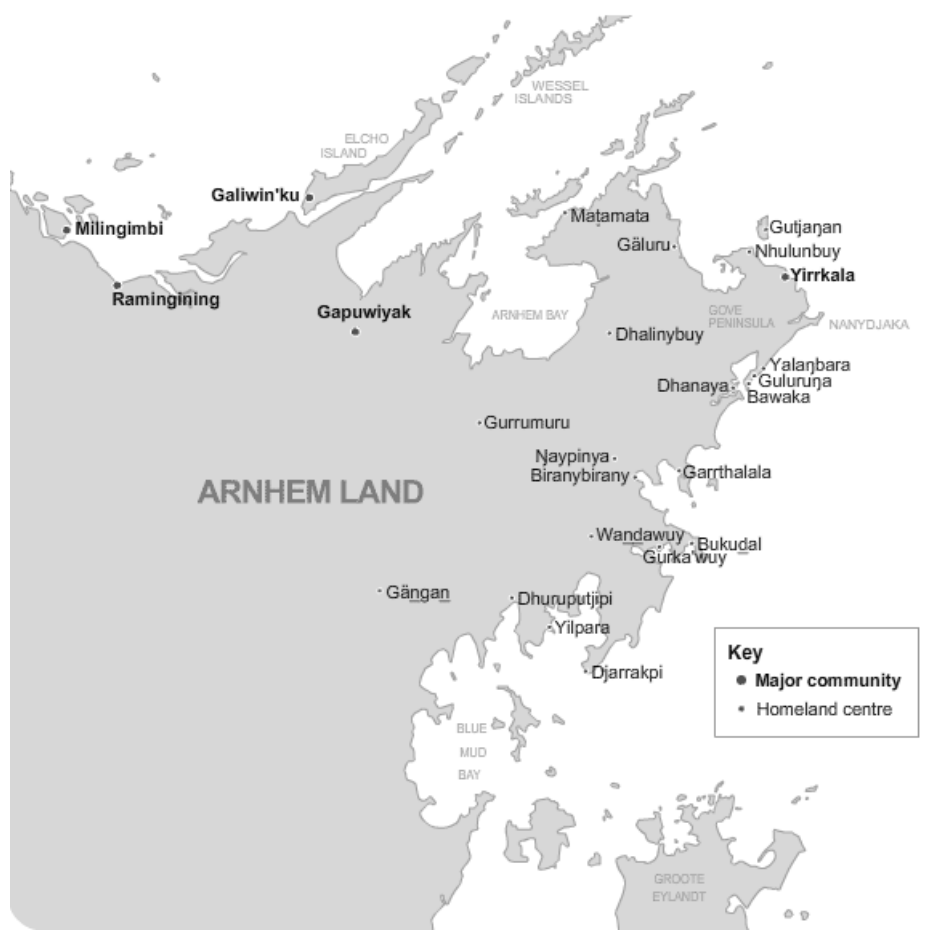

Galiwin'ku community started as a Methodist (now Uniting Church) mission in the 1930s. Today together with Yirrkala and Milingimbi communities it is one of the three largest Yolngu communities in Arnhem Land.

Officially there are 5,000 speakers of Yolngu languages scattered across Arnhem Land, with 2000 in Galiwin'ku. This number can shrink between 800 and 900 in the dry season when the indigenous people are very mobile. 
The population speaks Djambarrpuyngu, one of the Yolngu Matha languages. The community is multilingual with speakers of other Yolngu Matha language groups: Gupapuyngu, Gumatj, Liyagawumirr, Datiwuy, Galpu, Golumala, Rittharngu, Warramirri, Wanguri, Dhaluwangu. The community uses both Djambarrpuyngu and YSL to communicate and can be regarded as a bimodal bilingual community (Maypilama \& Adone 2012). Some balanda 'white' people working as shopkeepers, policemen, teachers, doctors in the community also use some of the basic signs to communicate with the deaf and hearing Yolngu people.

In 1994 there were five deaf people on the island (Cooke \& Adone 1994). In 2012 we identified 7 deaf people in the community; one male child around four, two female adults, between forty and fifty, one male adult in his late twenties, one male adult in his late sixties, and two teenagers. Based on the information provided by the health clinic in July 2012 and Butcher's work (p.c) we expect a high number of hearing-impaired members of the community. There is no information is available on the pattern of deafness in the community and it does not seem to be a link between deafness and surnames. As far as occupation is regarded, there is a deaf man working as a painter, a deaf woman working in craftsmanship.

Deaf people seem to be well integrated into the community. Evidence for this comes from the high level of involvement on their part in serious discussions regarding the community, as well as their leading function in ceremonies and other social tasks. There is no negative attitude towards YSL and the deaf members of the community. YSL is regarded as another Yolngu language like the other Yolngu languages mentioned earlier in this paper. It appears the language is severely endangered for reasons discussed in Maypilama \& Adone (2011). Although indigenous people are known to be very mobile in the dry season, none of the deaf people in the community have travelled further south to e.g. Central Australia where Kendon (1988) identified sign languages. However both Yolngu hearing and deaf Yolngu people have contact with people using Tiwi hand signs and signs from West Arnhem Land.

Some of the deaf Yolngu people have attended primary school together with hearing children. But there is no school for the deaf in the community. Recently a teacher of AUSLAN has been teaching the male child AUSLAN and signed English. 


\section{References}

Cooke, M. \& D. Adone

1994 Yolngu signing -gestures or language? In: CALL working papers. Batchelor. Australia.

Kendon, A.

1988 Sign languages of Aboriginal Australia. Cambridge. Cambridge University Press.

Maypilama, E.L. \& D. Adone

2011 Assessing Language Endangerment: The Case of Yolngu Sign Language. Unpublished manuscript. Charles Darwin University and University of Cologne.

Maypilama, E.L. \& D. Adone

2012 The Sociolinguistics of Yolyu Sign Language. Paper given at TELC, Charles Darwin University. Darwin. Australia. 


\section{Language index}

Aboriginal English $\quad 57,60$

Aboriginal languages $\quad 68,79,314$

ABSL (see Al-Sayyid Bedouin Sign Language)

Adamorobe Sign Language (AdaSL) 4, 10f, 15-17, 27ff, 54f, 72, 117, 189, 191-193, 196, 257, 347ff, 355

Akan 38, 196, 347f, 350

Algerian Jewish Sign Language (AJSL) 3, 4, 15, 153ff, 361ff,

Alipur Sign Language (APSL) 4, 12, 329, $353 \mathrm{ff}$

Al-Sayyid Bedouin Sign Language (ABSL) 4, 7, 16, 18, 55, 87ff, 154, 201, 215, 270, 365ff

American Sign Language (ASL) 9f, 54, 69f, 127f, 136, 139f, 147, 184-187, 191, 193, 196-203, 231, 234, 237, 241, 253-256, 259, 286, 297f, 350, 356, 358f, 387f, 390-392

Arabic 17, 90, 98f, 101-108, 110f, 114f, 165, 242, 362, 366-368, 398

Auslan (see Australian Sign Language) Australian Kriol language (see Kriol)

Australian Sign Language (Auslan) 5, 261, 402

APSL (see Alipur Sign Language)

Balinese 7, 17, 132, 135, 381, 383f

Ban Khor Sign Language (BKSL) 4, 8, 54f, 131, 203, 277ff, 373ff

Brazilian Sign Language (Libras) 10

British Sign Language (BSL) 9, 196f, 391
Chican Sign Language (see also Yucatec Mayan Sign Language) 5, 12, 314-316, 324, 331, 333, 336, $355,377 \mathrm{ff}$

Country Sign (see Konchri Sain)

Dhuwal 57, 59

Djambarrpuyyu 57, 59f, 63-66

Djapu 57

Dogon 252, 257f, 260f

Dutch 29, 181, 192f, 197, 384

Eesti viipekeel (see Estonian Sign Language)

English (see also Manually Coded English, Signed English) 31, 38, 57, 59f, 68-72, 76-78, 128, 133f, 147, 184-187, 192f, 198, 200-203, $210,217,226,231,235,241,255$, 293, 313, 324, 331, 354, 357, 377, 387f, 391, 401f

Estonian Sign Language $\quad 54$

Ghanaian Sign Language (GSL) 4, $31,32,35,349,350$,

Greek Sign Language (GSL) 193

Gupapuyngu 57, 402

Gumatj 57, 402

Hebrew 90f, 99, 100, 104-107, 110f, 114f, 160, 165-167, 242, 362f, 366-368

Indo-Pakistani Sign Language (IPSL) 17, 196 
Indo-European languages $\quad 9,242,244$ International Sign (IS) $\quad 10,313,335$

Inuktitut $\quad 182-186,188,192 f, 195$, 198-203, 389, 391

Inuit Sign Language (IUR) 5, 6, $181 \mathrm{ff}$

Israeli Sign Language (ISL) 110, 118, 153f, 166, 241f, 363, 366

Jamaican Creole (see Patwa)

Jamaican Sign Language (JSL) 5, 53ff, $387 \mathrm{f}$

Japanese Sign Language (JSL,

NS) 10

Jordanian Sign Language (LIU) 103, 106

Kata Kolok 5, 10f, 15-18, 54, 127ff, 201, 203, 226, 234, 240-242, 355, $381 \mathrm{ff}$

Konchri Sain $\quad 5,17,53 \mathrm{ff}, 387 \mathrm{f}$

Kriol 57

Kurdish $\quad 17,398$

Langue des Signes Québécoise, LSQ (see Quebec Sign Language)

Lao 282, 373

Lengua de Señas Mexicana, LSM (see

Mexican Sign Language)

Libras (see Brazilian Sign Language)

LIU (see Jordanian Sign Language)

Língua Brasileira de Sinais (see

Brazilian Sign Language)

LSM (see Mexican Sign Language)
LSQ (see Quebec Sign Language)

Lughat il-Ishaarah il-Urduniah, LIU (see Jordanian Sign Language)

Manually Coded English $\quad$ 184, 187, 198, 391

Mardin Sign Language (MarSL) 3, 5, 12, 17 271, 313ff, 355, 395ff

Mexican Sign Language (LSM) 215, 315f, 323, 379,

Mian 200

Nederlandse Gebarentaal, NGT (see Sign Language of the Netherlands)

Nihon Shuwa, NS (see Japanese Sign Language)

North Central Desert area sign languages 57

Nyoh 373

Pasa Kidd (see Ban Khor Sign Language)

Pasa Bai (see Ban Khor Sign Language)

Patwa (Jamaican Creole) $\quad 68-70,72$, $387 \mathrm{f}$

Phuthai 373

Plains Indian Sign Language (PISL) 6, 57, 183, 390

Providence Island Sign Language

(PROVISL) 55, 191

Qderat 102, 367

Quebec Sign Language (LSQ) 197, 391 
Russian Sign Language (RSL) 155, 170, 171

Sign Language of the Netherlands (NGT) 127, 189, 192f, 197, 201, 261, 321

Signed English $\quad 69,255,387 f, 402$

Soe 373

Spanish 211-213, 226, 228, 243f, $324,331,336,341,377,379$,

TID (see Turkish Sign Language)

Thai 282,373

Thai Sign Language (TSL) 7, 292ff, 373

Tiwa 314
Türk İşaret Dili (see Turkish Sign Language)

Turkish 3, 17, 193, 317, 320, 325f, $335,395 \mathrm{ff}$

Turkish Sign Language (TiD) 5, 10, 193, 317, 320, 325f, 335, 395ff

Twi (see Akan)

Warlpiri 200, 241,

Yolngu 53ff, 401ff

Yolngu Sign Language (YSL) 53, 56, 64, 401ff

Yucatec Maya 17, 209ff

Yucatec Mayan Sign Language

(YMSL) 5, 17, 209ff, 315 


\section{Subject index}

acquisition (see language acquisition)

agreement verbs (see verb agreement)

alternate sign language (see sign

languages)

anonymity $32,47,183,203,324,390$

anthropology 2, 6, 19, 27-32, 45,

$47-48,53,89,118,133,188$,

278-279, 281-283, 286, 300, 319, 374-375

aspect (see imperfective aspect,

perfective aspect)

attrition, L1 (see L1 attrition)

bilingualism (see also sign

bilingualism, multilingualism) 53, 70, 78-79, 88, 98f, 104f, 110f,

115-118, 31, 143, 153, 159, 168, 172, 174, 184-187, 198, 200, 211, 213, 226, 228, 229, 240-242, 255, 257, 328-329, 350, 363, 387-388, 398,402

bimodal 111, 116, 213, 402

unimodal 111

and literacy $102 \mathrm{ff}, 162,166,169$, 268, 332-333, 355, 357-359, 366

and schooling 96ff, 174, 184, 211, 213f, 255, 257, 328, 350, 363f, $387 f$

borrowing (see loan words)

classifiers 196,295

(whole) entity 10f, 18, 191f, 227

co-inquiry (see also participatory research) 331

code-switching 111, 172, 296, 299

cohort (see also generations of

signers) 4f, 18, 89ff, 130, 214 colour (see also primary colours) 15-17, 53ff, 200f, 203, 212f, 243, 378,396

community engagement (see also research ethics) $313,317 \mathrm{f}$, 327-334

Community-Based Participatory Research (see participatory research)

conflict management $35,326 f$, 335-337

cultural conflict (see also Deaf culture) 327,335

completive aspect (see also perfective aspect) $135,217,218,234$

consanguinity (see also intermarriage, kin endogamy) 90, 93, 284

consent (see informed consent) contact language (see language contact)

corpus/corpora 129f, 132-135, 137-140, 143f, 146f, 190, 202, 220, 252, 254, 257, 259, 260f, 268, 270f, $321,374 \mathrm{f}$

Cross-modal contact (see also language contact) $6,15,181,195,198,398$

Deaf associations $\quad 15,256,316$

Deaf culture (see also cultural conflict, Deaf community) 27, 29, 193, 392

Deaf club (see Deaf associations)

Deaf community (see also Deaf culture) 3, 7, 29, 47, 131, 153-155, 164-170, 173-176, 191, 203, 211f, 251-255, 267-270, 277, 279f, 289, 292, 297, 299, 318, 321, 331, 363f, 373, 388, 398 
deaf education $\quad 4-6,15,40,55,69$, 80, 96, 103f, 115, 132, 154, 166f, 198, 251-256, 268, 270, 272, 292, $328,350,360,367-369,388,391$, 398

deaf-deaf relationships $7,30,36,39$, $43,100,110,112,117,162,176$, $271,348,350,369 f$

deaf-hearing relationships $7,30,36$, $37,39,162,271,290$ f, 348-351

Deaf-led research (see also research ethics, community engagement) 28, 35, 39, 314, 319f, 337f, 339, 340, 357

Deaf researchers (see Deaf-led research)

"deaf same" 34f, 43, 47f, 392 deaf village $2 \mathrm{f}, 28,42,45,48,130$, 140, 148, 267, 283f, 299, 313, 316, 347-351, 354-357, 377-379, 381-383, 385

demography $2-6,8,89,130,157 f$, 170-172, 181, 191, 203, 277, 279f, 282, 289, 291, 347, 356, 363, 365, 375,381

developing countries $46,253,314$, 341

development projects $\quad 43-45,47,350$

diaspora 3,320

dictionary (see sign language dictionary)

directional verb (see verb agreement) directionality (see verb agreement)

discourse 10f, 32, 34, 39, 41, 43, 48, 93, 136f, 139, 141, 218-220, 224, 227, 234, 239f, 254, 293, 314, 341, 349,374 , discourse referent 10f, 193, 222

documentary linguistics (see language documentation)

domains of language use $4 \mathrm{f}, 6,78$, $90,132,172,183,278,281,347$, 367

education (see deaf education)

emerging sign languages (see sign languages)

endangered languages (see language endangerment)

ethics (see research ethics)

ethnolinguistic vitality (see language vitality)

evidentiality 9

facial expressions (see non-manual signals)

family sign language $\quad 3,267,271 \mathrm{f}$ fieldwork (see also research ethics, reciprocity, researcher reception, snowball sampling) 2, 19, 30-33, 36, 39f, 58, 66, 89f, 129f, 132, 134, 182, 187f, 193, 212-215, 317f, 320, 322, 326, 331f, 334, 340, 342, 356f, 359,392

first language acquisition (see language acquisition)

gatekeepers 31, 326f, 329

generations

of signers (see also cohort)

3-5, 87, 88, 92ff, 214115, 117, 214

structural, of descent $89,92 \mathrm{ff}$

socio-cultural $89,92 \mathrm{f}, 95$ 
gesture (see also non-manual signals) $17,19,32,41,88,114$, 127f, 135, 154, 158, 181f, 191-195, 202, 209f, 213f, 217, 220-228, 230-232, 234, 236, 239-243, 253, 264, 267-269, 296, 323, 332, 335, 377

grammatical categories 9,11

grammaticalisation $11,135,241 \mathrm{f}$

handshape $11,60-63,75,81,108$, 189, 192, 196f, 199, 202, 245, 255, 293, $296 \mathrm{f}$

hearing signers (see also L2 sign language users) 4f, 8, 15-17, 59, 88f, 92, 97-99, 102f, 110-112, 114f, 121, 130-132, 164, 173f, 183, 191f, 202, 258, 268-270, 288, 299, 342, $356,365-370$

home sign $16,18,167,174,243$, $251,265,267-272,283,355$

iconicity (see also semantic motivation) 54, 63, 78, 136, 191f, 228f, 230, 240, 259, 367

identity $27,47,168,170 \mathrm{f}, 173 \mathrm{f}$, 338-f, 392

immigration (see migration) imperfective aspect 129, 134f, 146, 218

incidence of deafness $7,15,69,130 \mathrm{f}$, 162, 191, 252f, 262f, 268, 270-272, $354,377,388$

indigenous $3,7,53,55,57,68,78$, 130, 212, 277, 279, 297, 313, 353, $356,365,373,387,401 \mathrm{f}$

Indo-European languages (see Language Index) informed consent $28,30-33,48,160$, $187,318,320-326,336,342$

intergenerational transmission 6,19 , $88,92,97,102,115,117,215,269$, 283

intermarriage (see also consanguinity, kin endogamy) 90,365

interpreting 7, 15,31f, 38, 57, 89, 91, 106, 185f, 255, 286, 298, 316, 323f, 326f, 331, 336, 338, 341, 364, 367, $379,385,388,391 \mathrm{f}$

interrogative $9,17 \mathrm{f}, 127 \mathrm{f}, 136,143$, 293, 374

isolation (see social isolation)

kin endogamy (see also intermarriage, consanguinity) $90,93,365 f$

kinship diagrams $\quad 89,92-95,117 f$, 396

\section{L1 attrition 155,170}

L2 sign language users (see also hearing signers) 192

labour patterns $111-114,156,162$, 164, 185, 211-213, 262-266, 284, 288, 290, 298f, 328, 350, 354, 356-358, 366, 369, 374, 378, 383, 392,402

language acquisition $31,57,60$, 97, 99, 107, 109, 117, 127-135, 139-141, 144-148, 163-165, 174, 185-186, 213, 240, 267, 272, 286, 288-292, 300, 357, 362, 374f, 377, 391,395

first language acquisition $\quad 19,107$, 130,131

second language acquisition 16 , 97, 155, 191f, 202f 
language attitude $\quad 69,333 \mathrm{f}, 387 \mathrm{f}$

language contact $4 \mathrm{f}, 7,16,172,181 \mathrm{f}$, 184, 195, 198, 278, 296f, 299, 356, 359

language documentation (see also fieldwork, snowball sampling) 19, $254,314,318,320,329,330,359$

language endangerment (see also moribund language) $3-5,19,69 \mathrm{f}$, 169, 203, 254, 261, 277, 281f, 300, 329, 340f, 358f, 375

language prestige 173

language resources (see also multimedia materials, sign language dictionary) $316,333 \mathrm{f}$

language vitality $156,170,172-175$, 279, 359

literacy $31,102 \mathrm{ff}, 132,162,166$, $169,268,332 f$, 355, 357-359, 366, 369

loan translation (see also borrowing) 195-196, 198, 200-203

loan words (see also borrowing) 196, 243

localisation $10-11,193,209,223$

locus/loci 11

\section{macroethics 323}

marriage patterns (see also deafdeaf relationships, deaf-hearing relationships) $7,66,90 \mathrm{f}, 112 \mathrm{f}$, 116f, 130, 157, 161f, 164, 169, 176, 284, 287, 299, 347-349, 351, 354, $357,363,365,369 f$

mediators $98,326 f, 330$

micro-community sign language (see sign languages) microethics 323

migration $3-5,112,116,159,165 f$, $169,289,347,349,363,388$

mirativity 9

modality (see also cross-

modal typology, multimodal communication) $6,10,15 \mathrm{f}, 19 \mathrm{f}$, 80, 111f, 116, 130f, 147f, 191, 198, 213, 239f, 272, 338, 402

multimodal communication $\quad 19,213$ monolingualism (see also sign monolingualism) 5, 69, 162, 165, $169,172,174,184 f, 187,190,199$, 202, 211, 213, 355, 362, 388, 398

moribund language (see also language endangerment) 5, 169, 297

mouthing (see also non-manual signals) $17,60 f, 65,69,71-73$, $76,79,121,132,135 \mathrm{f}, 186,188$, 195, 197f, 201-203, 255

multilingualism (see also bilingualism) 57, 69, 110f, 118, $165,170,253,256 f, 286,373,391$, 398,402

multimedia materials (see also language resources, sign language dictionary) 329

negation $18,127 f, 135,137,193 f$ networks (see social networks) non-governmental organisations

(NGOs) 28, 40-43, 256f, 349f non-manual signals (see also facial expressions, mouthings) 17 , 79, 127-129, 135-141, 143-149, 192-195, 202, 232, 296

number (see numerals) 
numerals 12f, 16f, 20, 194f, 199, 201f, 227, 230f, 233, 241f, 292, 323, 398

cardinal 12, 194f, 199, 323, 398

ordinal $194 \mathrm{f}$

and spatial morphology $\quad 12-14$

subtractive $12,14,17$

vigesimal 12,17

numeral bases 12,13

base-20 (see vigesimal numerals)

base-50 12

participant observation $28,32,38$, 89-90, 348

participant voice 314,341

participatory research (see also coinquiry) 329,331

Participatory Action Research (see participatory research)

perfective aspect (see completive aspect) 127-130, 134-137, 140, 144, 147,

pidgin 10,297

pointing $11,16,35,54 \mathrm{f}, 61,63-66$, 68, 70, 74f, 79f, 129, 137, 139, 140-144, 147, 203, 222, 226f, 229, 230, 232, 264f, 367

possession $9,18,381$

prestige (see language prestige)

primary colours (see colour)

questions (see interrogative)

reciprocity (see also research ethics)

referent (see discourse referent) religion $35,38,132,157,165,282$, $329,335,338,350,353-354,362$, 378,383

researcher effects $28,35,36,48$

research ethics (see also reciprocity, community engagement, Deaf researchers)

researcher reception (see also fieldwork)

rural sign languages (see sign languages)

sampling (see snowball sampling)

schools for the deaf (see deaf education)

second language acquisition (see language acquisition)

semantic fields 9, 15

semantic motivation (see also iconicity) $73 \mathrm{f}$

serial verb construction 17

shared signing communities (see also shared sign languages) 2f, 6-9, 27f, 30, 46f, 87-89, 93, 97, 110f, 115f, 118, 345f, 367

sign bilingualism (see also bilingualism) 78f, 171

sign monolingualism $172,174,398$ sign language dictionary 297, 298, 331,379

sign language interpreting (see interpreting)

sign languages (see Language Index for specific sign languages) alternate $3,5,57,78,81,183,390$ emerging 3,87 
endangered $3,7,19,131,341$

extinct 297

microcommunity $268,271 \mathrm{f}$

rural $3,6-8,15-19,55,67,79$, $318,356,387$

shared (see also shared signing communities) 2f, 6-9, 87-89, 97, $115,117 \mathrm{f}$

urban $2,6-11,15-19,55,79 f$, 191f, 203, 277, 317, 356, 358

village $2 \mathrm{ff}, 53,55,79 \mathrm{f}, 127,129$, 134, 148, 154, 182, 191f, 196, 201, 203, 210, 242, 268, 277-280, 282, 286, 300, 313-315, 323, 338, 341, $353,356,361,373-375,377$

signing space $10 f, 15,91,189,192$, 296

snowball sampling $\quad 252,258,271$

social isolation $157,171,353,361$

social networks

kin and non-kin $55,98,111,116$, 172f, 263-266, 291, 299, 359, 365, 369,375

and labour 111f, 116, 268

and schooling $7,89 f$, 96ff

space (see signing space)

spatial loci (see loci)

spoken languages (see Language Index

for specific spoken languages)

stigmatisation $168 \mathrm{f}, 175,284,363$, 368,383 sociolinguistics $30,36,55 f, 58,68$, 87-89, 96, 98f, 102f, 105-107, 109f, 112-118, 129, 155, 182f, 191, 209f, 213, 243, 251f, 262, 267f, 271f, 277f, 285f, 300, 313, 347f, $353,355,361,365,370,373,377$, $381,384,387,389 f 401$

technology $318,320,329,331 \mathrm{f}, 337$, $379 f$

time, expression of $19,133 \mathrm{ff}, 209 \mathrm{ff}$ typology $3,8-19,53,78,80,128 f$, 134, 147f, 202, 244, 277f cross-modal (see also modality) of sign languages $3,8-19,53$, 134,244

of spoken languages $\quad 9-15,244$

urban sign languages (see sign languages)

variation

linguistic 8-19

sociolinguistic 155

typological $\quad 8-19$

verb agreement $10,18,242$

village sign languages (see sign languages)

virtue ethics 324

vitality (see language vitality) 